\title{
Novel 3D Ultrasound Elastography Techniques for In Vivo Breast Tumor Imaging and Nonlinear Characterization
}

\author{
Ahmed A. Hameed Sayed \\ West Virginia University
}

Follow this and additional works at: https://researchrepository.wvu.edu/etd

\section{Recommended Citation}

Sayed, Ahmed A. Hameed, "Novel 3D Ultrasound Elastography Techniques for In Vivo Breast Tumor Imaging and Nonlinear Characterization" (2013). Graduate Theses, Dissertations, and Problem Reports. 5001.

https://researchrepository.wvu.edu/etd/5001

This Dissertation is protected by copyright and/or related rights. It has been brought to you by the The Research Repository @ WVU with permission from the rights-holder(s). You are free to use this Dissertation in any way that is permitted by the copyright and related rights legislation that applies to your use. For other uses you must obtain permission from the rights-holder(s) directly, unless additional rights are indicated by a Creative Commons license in the record and/ or on the work itself. This Dissertation has been accepted for inclusion in WVU Graduate Theses, Dissertations, and Problem Reports collection by an authorized administrator of The Research Repository @ WVU.

For more information, please contact researchrepository@mail.wvu.edu. 


\title{
Novel 3D Ultrasound Elastography Techniques for In Vivo Breast Tumor Imaging and Nonlinear Characterization
}

\author{
Ahmed A. Hameed Sayed \\ Ph.D. Dissertation submitted to the \\ Benjamin M. Statler \\ College of Engineering and Mineral Resources \\ at West Virginia University \\ in partial fulfillment of the requirements \\ for the degree of

\section{Doctor of Philosophy} \\ in \\ Mechanical Engineering
}

Approved by

Osama Mukdadi, Ph.D., Committee Chair

Victor Mucino, Ph.D., Committee Member

Nithi T. Sivaneri, Ph.D., Committee Member

Terence Musho, Ph.D., Committee Member

Ginger Layne, M.D., Committee Member

Department of Mechanical and Aerospace Engineering

Morgantown, West Virginia

2013

Keywords: Breast Masses, Tissue Nonlinearity, Elastography, 3D Imaging, Segmentation.

Copyright 2013 Ahmed A. Hameed Sayed 


\title{
ABSTRACT \\ Novel 3D Ultrasound Elastography Techniques for In Vivo Breast Tumor Imaging and Nonlinear Characterization
}

\author{
Ahmed A. Hameed Sayed
}

Breast cancer comprises about $29 \%$ of all types of cancer in women worldwide. This type of cancer caused what is equivalent to $14 \%$ of all female deaths due to cancer. Nowadays, tissue biopsy is routinely performed, although about $80 \%$ of the performed biopsies yield a benign result. Biopsy is considered the most costly part of breast cancer examination and invasive in nature. To reduce unnecessary biopsy procedures and achieve early diagnosis, ultrasound elastography was proposed.

In this research, tissue displacement fields were estimated using ultrasound waves, and used to infer the elastic properties of tissues. Ultrasound radiofrequency data acquired at consecutive increments of tissue compression were used to compute local tissue strains using a cross correlation method. In vitro and in vivo experiments were conducted on different tissue types to demonstrate the ability to construct 2D and 3D elastography that helps distinguish stiff from soft tissues. Based on the constructed strain volumes, a novel nonlinear classification method for human breast tumors is introduced. Multi-compression elastography imaging is elucidated in this study to differentiate malignant from benign tumors, based on their nonlinear mechanical behavior under compression. A pilot study on ten patients was performed in vivo, and classification results were compared with biopsy diagnosis - the gold standard. Various nonlinear parameters based on different models, were evaluated and compared with two commonly used parameters; relative stiffness and relative tumor size. Moreover, different types of strain components were constructed in 3D for strain imaging, including normal axial, first principal, maximum shear and Von Mises strains. Interactive segmentation algorithms were also evaluated and applied on the constructed volumes, to delineate the stiff tissue by showing its isolated 3D shape.

Elastography 3D imaging results were in good agreement with the biopsy outcomes, where the new classification method showed a degree of discrepancy between benign and malignant tumors better than the commonly used parameters. The results show that the nonlinear parameters were found to be statistically significant with $\mathrm{p}$-value $<0.05$. Moreover, one parameter; power-law exponent, was highly statistically significant having p-value $<0.001$. Additionally, volumetric strain images reconstructed using the maximum shear strains provided an enhanced tumor's boundary from the surrounding soft tissues. This edge enhancement improved the overall segmentation performance, and diminished the boundary leakage effect. 3D segmentation provided an additional reliable means to determine the tumor's size by estimating its volume.

In summary, the proposed elastographic techniques can help predetermine the tumor's type, shape and size that are considered key features helping the physician to decide the sort and extent of the treatment. The methods can also be extended to diagnose other types of tumors, such as prostate and cervical tumors. This research is aimed toward the development of a novel 'virtual biopsy' method that may reduce the number of unnecessary painful biopsies, and diminish the increasingly risk of cancer. 


\section{ACKNOWLEDGMENTS}

I would like first and foremost to thank Dr. Osama Mukdadi, my Ph.D. adviser. I was fortunate to have Dr. Mukdadi as my adviser for patiently providing the encouragement, vision, and advice essential for me to complete my dissertation. His continuous sponsorship and support were necessary for the completion of this research. His detailed directions from the initial to final points enabled me to develop an in depth understanding of the research subject.

I also would like to express my gratitude to Dr. Ginger Layne for collaborating in this multidiscipline research. Discussions with Dr. Layne have provided me with very helpful and valuable knowledge on the medical aspects of the work.

Special thanks go to my Ph.D. examining committee members Dr. Victor Mucino, Dr. Nithi Sivaneri, and Dr. Terence Musho for their helpful suggestions, guidance, and service throughout the extent of my dissertation. I am also grateful to the fellows of the Biomedical Ultrasound Lab for their friendship and support.

This dissertation, as well as all my accomplishments would have not been achievable without the support from my family. My deepest appreciation goes to my parents for their continuous care and encouragement that allow me to pursue my goals. I also would like to thank my wife, for her unconditional support and sacrifices that have been instrumental in achieving my objectives. This work is dedicated to all of them.

Acknowledgement also goes to the National Institute of Health $(\mathrm{NIH})$ for the support received under grant \#5R21DE019561, and WV PSCoR. 


\section{Novel 3D Ultrasound Elastography Techniques for In Vivo Breast Tumor Imaging and Nonlinear Characterization}

\section{TABLE OF CONTENTS}

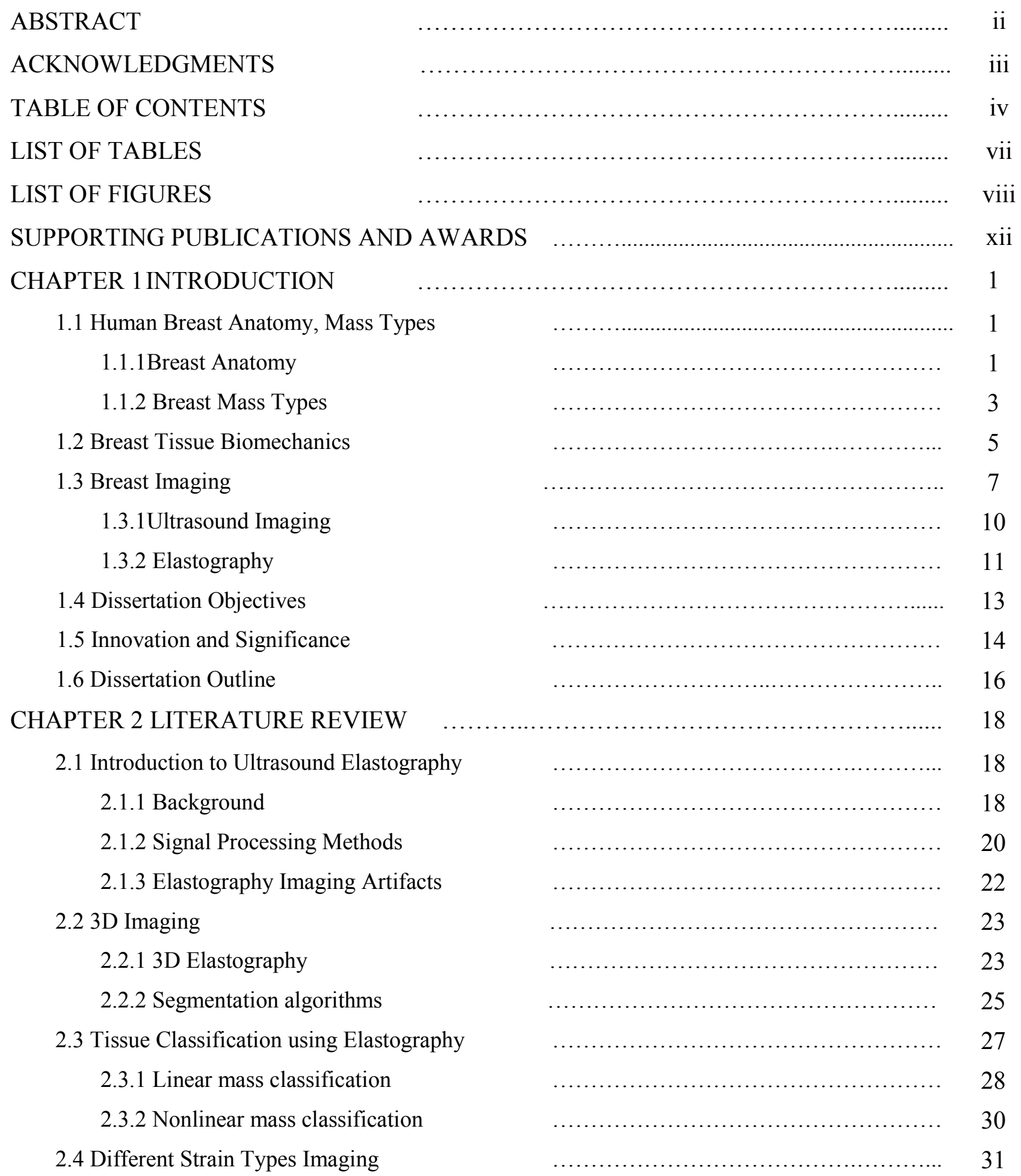


CHAPTER 3 MATERIALS AND METHODS

3.1 Ultrasound Signal Processing

3.1.1 Displacement Estimation

3.1.2 Estimation Noise

3.1.3 Displacement Values Correction

3.2 Image and Volume Constructions

3.2.1 Elastogram Construction

3.2.2 Derived Strains Construction

3.2.3 Volume Construction

3.2.4 Algorithm Summary

3.2.5 Graphical User Interface 44

3.3 3D Elastography, Lab Setup 46

3.4 3D Elastography, Clinical Setup 49

3.5 Evaluating Tissue Nonlinearity 52

3.6 Segmentation Algorithms 56

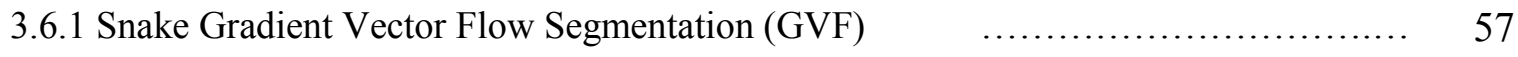

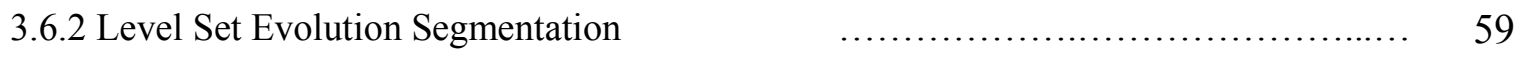

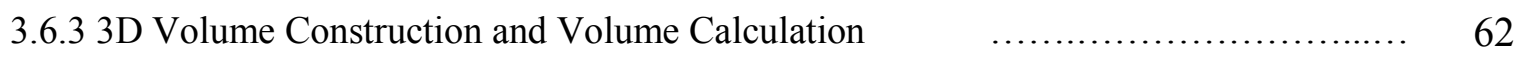

CHAPTER 4 TISSUE MIMICKING PHANTOM STUDY $\quad$......................... 63

4.1 Application of Elastography Technique 63

4.1.1 Breast Phantom 64

4.1.2 2D Elastography Imaging 64

4.2 3D Imaging 66

4.2.1 3D Elastography 66

4.2.2 Derived Strains Imaging 67

4.3 Quantitative Measures

4.3.1 Relative Stiffness 70

4.3.1 Inclusion Volume Calculation

4.4 Imaging Quality Assessment

4.4.1 Elastography Frames

4.4.2 Elastography Slices

4.5 Summary

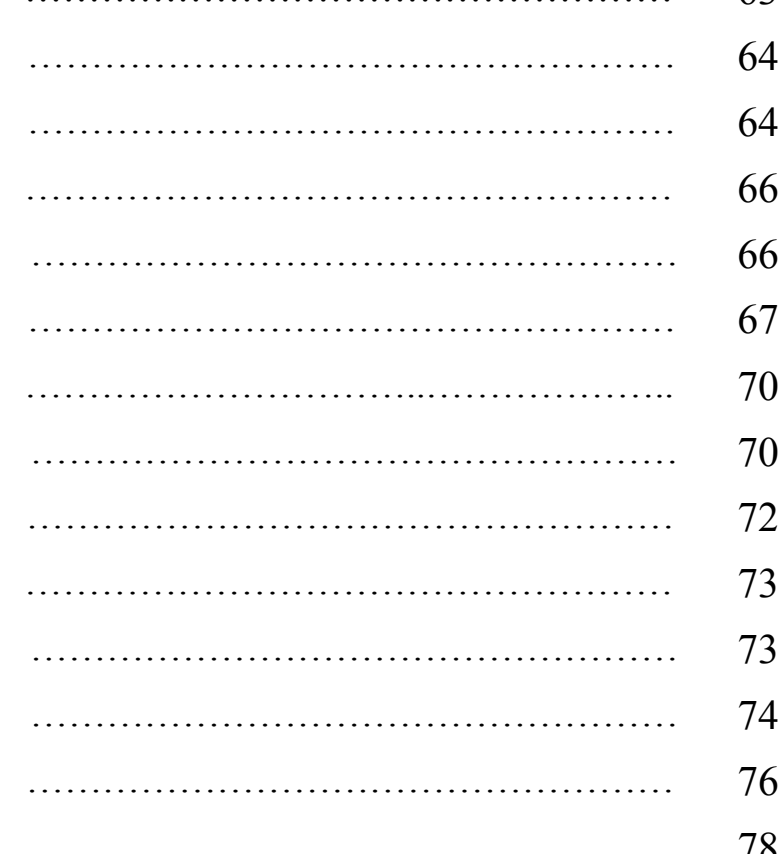

CHAPTER 5 IN VIVO CLINICAL STUDY 
5.1 Study Aims and Polices 78

5.2 3D Breast Mass Strain Imaging

5.2.1 Benign Cases 80

5.2.2 Malignant Cases 83

5.3 Mass Classification

5.3.1 Addressing Tissue Nonlinearity 85

5.3.2 Quantifying Degrees of Nonlinearity 85

5.3.3 Classification Methods Comparison 89

5.3.4 Repeatability Study 92

5.4 Derived Strains Imaging 96

5.5 Study Summary

100

CHAPTER 6 TUMOR SEGMENTATION

6.1 Applying Segmentation

6.2 Evaluating Segmentation Algorithms

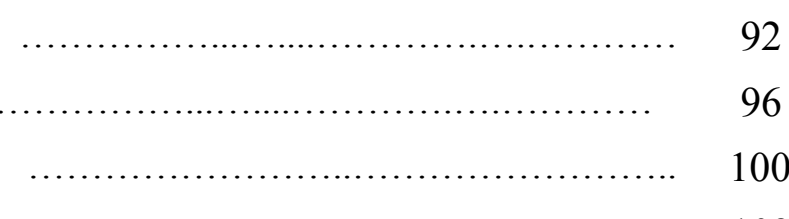

6.3 3D In Vitro Segmentation 103

6.4 3D In Vivo Segmentation

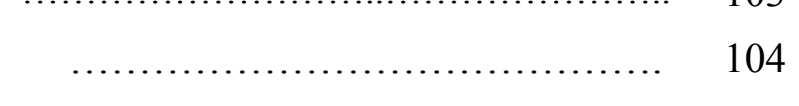

6.4.1 Normal Axial Strain Imaging

................................... 104

6.4.2 Derived Strains Effect

106

108

110

110

114

CHAPTER 7 CONCLUSION AND FUTURE WORK

7.1 Concluding Remarks

7.2 Future Work

121

REFERENCES 


\section{LIST OF TABLES}

Table 1.1 Main breast mass types.

Table 1.2 Breast imaging modalities comparison.

Table 4.1 Relative stiffness calculations.

Table 4.2 Inclusion volume calculations.

Table 5.1 Nonlinear parameters estimated for all masses.

Table 5.2 Mean and standard deviation values of the mass's classification parameters.

Table 5.3 Mass classification using relative stiffness.

Table 5.4 Mass classification using relative volume parameter.

Table 5.5 Statistical significance of the mass classification parameters.

Table 5.6 Mass classification methods comparison.

Table 5.7 Repeatability of the nonlinear parameter using double volumetric acquisitions for the same patients.

Table 5.8 Repeatability of the mass's volume calculation $\left(\mathrm{mm}^{3}\right)$ using double volumetric acquisitions.

Table 5.9 Calculation of the nonlinear parameter $n$ based on multiple 2D readings.

Table 5.10 Breast mass volume $\left(\mathrm{mm}^{3}\right)$ calculations for all cases using manual segmentation.

Table 6.1 Phantom's inclusion volume estimations using interactive segmentations.

Table 6.2 Breast mass volume $\left(\mathrm{mm}^{3}\right)$ calculations for all cases using interactive segmentation. 


\section{LIST OF FIGURES}

Figure 1.1 Human breast anatomy.

Figure 1.2 General process for cancer formation.

Figure 1.3 a) Normal cells, b) Cancer cells.

Figure 1.4 Measured stress-strain curves for eight different kinds of breast tissue.

Figure 1.5 Breast tissues simplified biomechanical behavior.

Figure 1.6 Schematic representation of current approaches to elastographic imaging.

Figure 2.1 Elastography technique: a) Tissue compression. b) Acquisition of pre- and postcompression signals. c) Cross-correlation function.

Figure 2.2 Examples of 3D elastography in the literature: a) Patil et al. 2007, b) Bharat et al. 2008, c) Deprez et al. 2009, d) Housden et al. 2010.

Figure 2.3 Application of a segmentation algorithm on 3D elastography data.

Figure 2.4 B-mode image and elastogram of cancer.

Figure 2.5 Breast cancer imaging using elastography: Sonogram and elastogram pairs from fibroadenoma and infiltrating ductal carcinoma.

Figure 2.6 Scatter plot of patient contrast values for two nonlinear parameters providing discrimination between benign and malignant masses.

Figure 2.7 a) Sonograms. b) normal axial elastograms. c) axial shear elastograms of a breast cancer.

Figure 3.1 3D ultrasound probe rocking motion.

Figure 3.2 Conceptual illustration of tissue displacement estimation.

Figure 3.3 Displacement image smoothing a) before smoothing $\mathrm{b}$ ) after smoothing.

Figure 3.4 Geometry illustrating the displacement image pixel values correction.

Figure 3.5 Scan conversion of a strain image: a) Before correction, b) After transformation, c) After interpolation.

Figure 3.6 Elastography flowchart.

Figure 3.7 Elastography GUI.

Figure 3.8 Elastography phantom experiment, Lab setup: a) schematic diagram of the components. b) photo image.

Figure 3.9 Clinical study setup: a) mammography paddle with an added holder for the 3D ultrasound probe. b) Ultrasound mammography system. 
Figure 3.10 Elastography multi-compression cycle.

Figure 3.11 General stress-strain difference relationships for benign and malignant masses. This relationship was plotted using in vitro mechanical measurements data.

Figure 3.12 Nonlinear classification of breast masses, main hypotheses.

Figure 3.13 Tissue compression method.

Figure 4.1 Breast phantom construction.

Figure 4.2 2D Breast phantom imaging.

Figure 4.3 2D and 3D Breast phantom imaging: a) Conventional B-mode slice. b) Axial strain elastographic slice. c) Conventional B-mode volume. d) Axial strain elastographic volume.

Figure 4.4 3D Breast phantom imaging using different volumetric strain types. The colorbar represents normalized strain.

Figure 4.5 Strain profiles across breast phantom's inclusion.

Figure 4.6 Regions selected for quantitative analysis.

Figure 4.7 2D elastography imaging quality assessment: a) SNR. b) CNR.

Figure 4.8 3D elastography imaging quality assessment: a) SNR. b) CNR.

Figure 5.1 Case number 1, benign breast mass: a) conventional ultrasound, b) mammography, c) elastography image, d) elastography volume.

Figure 5.2 Case number 5, benign breast mass: a) conventional ultrasound, b) mammography, c) elastography image, d) elastography volume.

Figure 5.3 Case number 2, malignant breast mass: a) conventional ultrasound, b) mammography, c) elastography image, d) elastography volume.

Figure 5.4 Case number 8, malignant breast mass: a) conventional ultrasound, b) mammography, c) elastography image, d) elastography volume.

Figure 5.5 Breast 2D elastography imaging at three compression levels, where the colorbar represents strain difference.

Figure 5.6 Benign breast masses behavior.

Figure 5.7 Malignant breast masses behavior.

Figure 5.8 Examples of nonlinear parameters estimation using curve fitting.

Figure 5.9 Classification parameters performance comparison: a-f) Nonlinear classification parameters estimated using: a-b) power-law, c-d) linear, and e-f) logarithmic 
relationships. g-h) Conventional elastography mass classification using: g) Relative stiffness. h) Relative volume.

Figure 5.10 A plot showing the discrimination margin between benign and malignant masses using repeatable estimations of the nonlinear classification parameter $n$.

Figure 5.11 In vivo volumetric strain elastograms for a benign case: a) Normal axial strain, b) First principal strain, c) Von Mises strain, d) Maximum shear strain. The colorbar represents normalized strain.

Figure 5.12 In vivo volumetric strain elastograms for a malignant case: a) Normal axial strain, b) First principal strain, c) Von Mises strain, d) Maximum shear strain. The colorbar represents normalized strain.

Figure 6.1 Example curve evolution at different iteration stages: a) initial contour, b) 60 iterations, c) 120 iterations d) 200 iterations.

Figure 6.2 Segmentation performance comparison between the two segmentation methods. a-b) Phantom's inclusion segmented using DRLSE and GVF snake, respectively. c-d) In vivo breast mass segmented using DRLSE and GVF snake, respectively. a) and c) shows better segmentation using the DRLSE method.

Figure 6.3 Segmented derived strain elastograms for the breast phantom: a) normal axial strain, b) first principal strain, c) maximum shear strain d) Von Mises strain.

Figure 6.4 Summary of the benign mass's characterization: first column states the patient's age and biopsy diagnosis, second column shows 3D axial elastography, third column shows segmented 3D axial elastography, and fourth column states the estimated mass's volume.

Figure 6.5 Summary of the malignant mass's characterization: first column states the patient's age and biopsy diagnosis, second column shows 3D axial elastography, third column shows segmented 3D axial elastography, and fourth column states the estimated mass's volume.

Figure 6.6 Mass segmentation performance evaluated by an experienced radiologist.

Figure 6.7 Segmented derived volumetric strain elastograms of a benign case; fibroadenoma. Patient's age was 40 years. a) normal axial strain, b) first principal strain, c) maximum shear strain , d) Von Mises strain. 
Figure 6.8 Segmented strain elastograms corresponding to mass 1 in Fig. 9: a) normal axial strain, the segmentation contour shows some leakage beyond the mass's boundary, $b$ ) first principal strain, c) maximum shear strain d) Von Mises strain. No boundary leakage appears in the derived strains.

Figure 6.9 Segmented derived volumetric elastograms of a malignant mass; IDC. Patient's age was 65 years. a) normal axial strain; estimated volume: $624.60 \mathrm{~mm}^{3}$, b) first principal strain; estimated volume: $609.10 \mathrm{~mm}^{3}$, c) maximum shear strain; estimated volume: $602.90 \mathrm{~mm}^{3}$, d) Von Mises strain; estimated volume: $592.40 \mathrm{~mm}^{3}$.

Figure 6.10 Segmented strain elastograms corresponding to the mass in Fig. 11: a) normal axial strain, the segmentation contour shows some leakage beyond the mass boundary, $b$ ) first principal strain, a minor degree of boundary leakage appears, c) maximum shear strain d) Von Mises strain. No boundary leakage appears in the two latter strains. 


\section{SUPPORTING PUBLICATIONS AND AWARDS}

1. Ahmed Sayed, Mohamad Salkini, Stan Majweski, and Osama Mukdadi , "3D Ultrasound Elastographic Imaging of Prostate Cancer Characterization", presented at the ASME International Mechanical Engineering Congress and Exposition, Denver, Colorado 2011.

2. Ahmed Sayed, Ginger Layne, Jame Abraham and Osama Mukdadi, "3D Ultrasound Elastographic Imaging of Breast Cancer In vivo", accepted abstract, poster and presentation at the 2012 MAE Department Graduate Student Poster Competition, and won the second place.

3. Ahmed Sayed, Ginger Layne, Jame Abraham and Osama Mukdadi, "3D Ultrasound Elastographic Imaging and Characterization of Breast Cancer In vivo", accepted as a technical paper at the ASME International Mechanical Engineering Congress and Exposition, Houston, Texas 2012.

4. Ahmed Sayed, Ginger Layne, Jame Abraham and Osama Mukdadi, "Nonlinear Characterization of Breast Cancer using Multi-Compression 3D Ultrasound Elastography In Vivo”, Research paper, Journal of Ultrasonics, vol. 53, 979-991 July 2013.

5. Osama Mukdadi, Ahmed Sayed, Ginger Layne and Jame Abraham, "System and Device for Tumor Characterization Using Nonlinear Elastography Imaging”, US provisional patent, number 61/775,555, filed March 2013.

6. Ahmed Sayed, Ginger Layne, Jame Abraham and Osama Mukdadi, “3D Visualization and Nonlinear Tissue Classification of Breast Tumors using Ultrasound Elastography In Vivo", Research paper, Ultrasound in Med. and Biol. (Submitted). 


\section{CHAPTER 1 \\ INTRODUCTION}

\subsection{Human Breast Anatomy and Mass Types}

\subsubsection{Breast Anatomy}

Human breasts form in the upper region of the torso, on both the right and left sides. In women, the breasts contain mammary glands that secrete milk for feeding the infants. Breasts are overlaid over pectoralis major muscles. They extend from the chest ribs number two to six, in front of the rib cage. Therefore, the breasts cover most of the chest area and its walls. In front of the chest, the breast tissue has the ability to extend from the clavicle bone to the center of the sternum. From the side to the chest walls, the breast can extend through the armpit, and can reach the latissimus dorsi muscle, expanding from the lower part of the back to the humerus bone. The breast tissue is considered to be a mammary gland, which is an anatomically inhomogeneous organ composed of different types of tissue layers. Among various tissue types, the adipose tissue and the glandular tissue are the main two tissue types that affect the breast lactation functions [1].

From the morphological perspective, the breast is conically shaped, with the cone base laying on the chest wall, and the apex is located at the NAC's center (nipple-areola complex) or the nipple, as shown in Fig. 1.1. Superficial tissue layers are separated from the outer skin by 0.5 to $2.5 \mathrm{~cm}$ of subcutaneous fat, which is the adipose tissue. Cooper's ligaments are fibrous-connective tissues that function as suspensory ligaments. They extend from the superficial tissue layers to the skin cover. The adult woman breast contains 14 to 18 irregular lobes that congregate at the nipple in the form of 2.0 to $4.5 \mathrm{~mm}$ diameter ducts. Those lactiferous lobes are also called milk ducts. Milk ducts are enclosed with dense connective tissue that forms a support structure $[1,2]$. Inner surfaces of the milk ducts and milk lobules are enclosed by a single layer of epithelial cells and then another layer of myoepithelial cells. The epithelial cells' main function is to secret the feeding milk, while the myoepithelial layer's function is to contract, resulting in increasing the pressure on the duct walls, which pushes the milk to the duct opening at the nipple, where all the milk ducts meet [3]. The glandular breast tissue is supported with estrogen in a biomechanical way. As a result, when a woman gets older and reaches the menopause phase, her body estrogen level decreases. Then, the 
milk gland tissues thin and disappear. This leaves the breast with the composition of the adipose tissue, superficial tissue, cooper's ligaments, and the skin envelope [2].

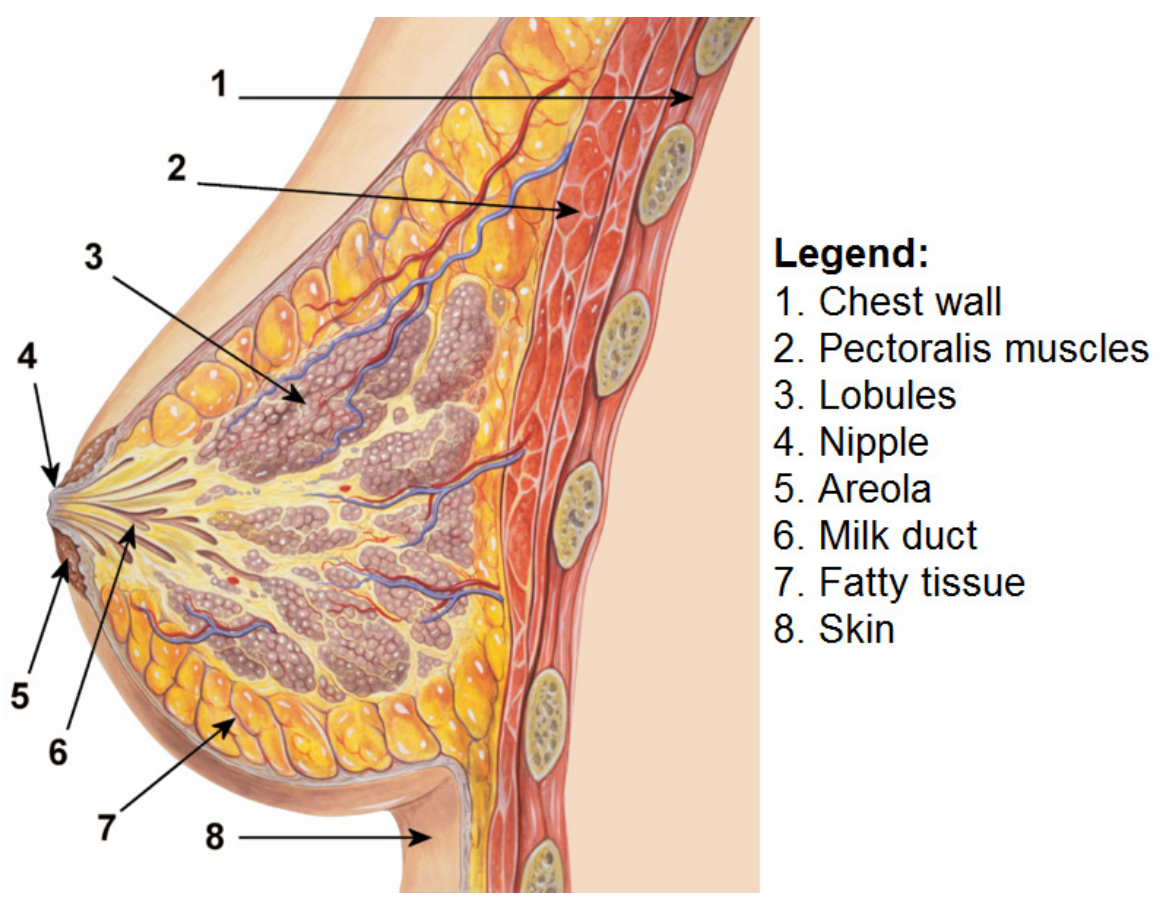

Figure 1.1 Human breast anatomy [4].

Breast dimensions and weight largely vary among women, ranging from 500 to 1,000 grams approximately for each breast. Breast tissue composition ratios vary as well among individuals. Some breasts have larger ratios of adipose tissue or fat than the ratios of glandular tissues, and so forth. In consequence, the ratio between the amount of fat-to-the connective-tissue determines the firmness or density of the breast. Through a woman's life, her breasts change in shape, size and weight, because of the changes in the hormonal levels that occur through different periods of the breast lifecycle, like menstruation, pregnancy and menopause [5].

From the Functional perspective, the breast as a whole is a gland that makes milk for the infant feeding purposes. Milk is delivered from the nipple of the breast, which is centered and surrounded by the breast areola, NAC. The basic functional breast units are named terminal duct lobular units (TDLUs), which produce the fatty breast milk and are distributed throughout the breast tissue. Two thirds of the lactiferous tissues are located within the $30 \mathrm{~mm}$ region of the base of the nipple, approximately. Milk is drained from the TDLUs into 4 to 18 lactiferous ducts, which in turn direct 
the milk to the nipple. In a lactating woman, the milk producing glands to the fat ratio is $2: 1$, while the ratio decreases to $1: 1$ in a non lactating woman $[1,6]$.

\subsubsection{Breast Mass Types}

Generally speaking, cancer happens when specific cells in some organ of the body start to grow in an uncontrollable way. Normal cells divide and proliferate in an organized manner, but malignant cells do not follow that order. Malignant cells persist to produce themselves uncontrollably, and disturb the surrounding normal cells, as in Fig 1.3.b. The continuously multiplying cells usually increase in size (hyperplasia) and then change their internal structure (metaplasia) to form a lesion that is called a tumor [7]. Yet, not all tumors are malignant or cancerous. Non cancerous tumors are called benign tumors or lesions. Benign tumors' cells do not spread to other organs or tissues, while cancerous tumors cells, or malignant tumors, can spread away from the original main tumor [8]. Malignant tumors can spread through blood circulation or the lymphatic system to other parts of the body. Malignant tumor cells settle and proliferate in new sites in a process called metastasis [3]. Figure 1.2 summarizes the steps involved in cancer formation.

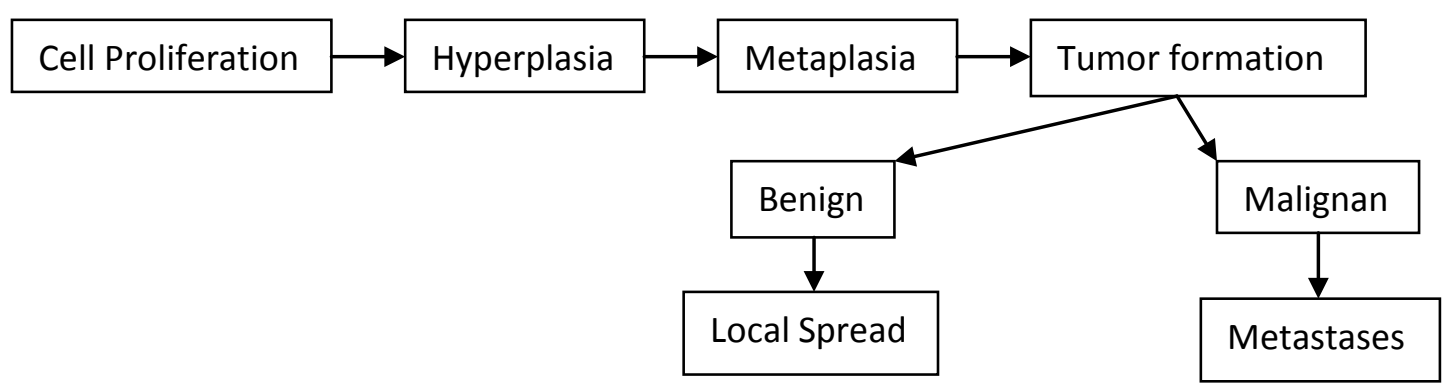

Figure 1.2 General process for cancer formation.

Regarding the breast tissue, the development of cancer within a mammary duct is demonstrated in Fig. 1.3. A large percentage of all breast cancer types start in ductal tissues. Genetic changes or environment variations to the epithelial cells make them develop and grow uncontrollably, forming malignant masses [3]. Breast malignant masses are usually harder and denser than the surrounding tissue, because of the uncontrollable way of growth and the increased blood supply. This characteristic facilitated cancer diagnosis using elasticity imaging techniques like ultrasound elastography. Many other causes of breast cancer also occur, but this is out of the scope of this 
dissertation, and a complete description of breast cancer types can be found in medical references $[7,9]$.

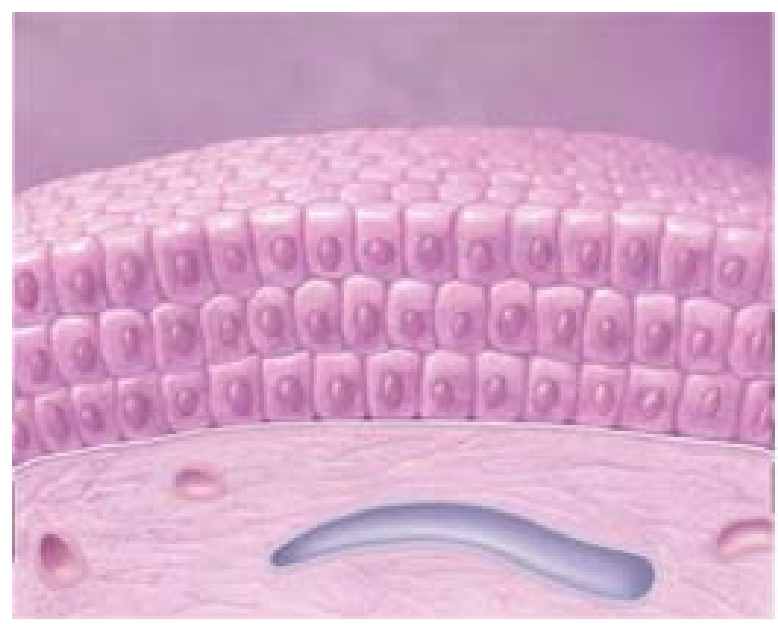

a)

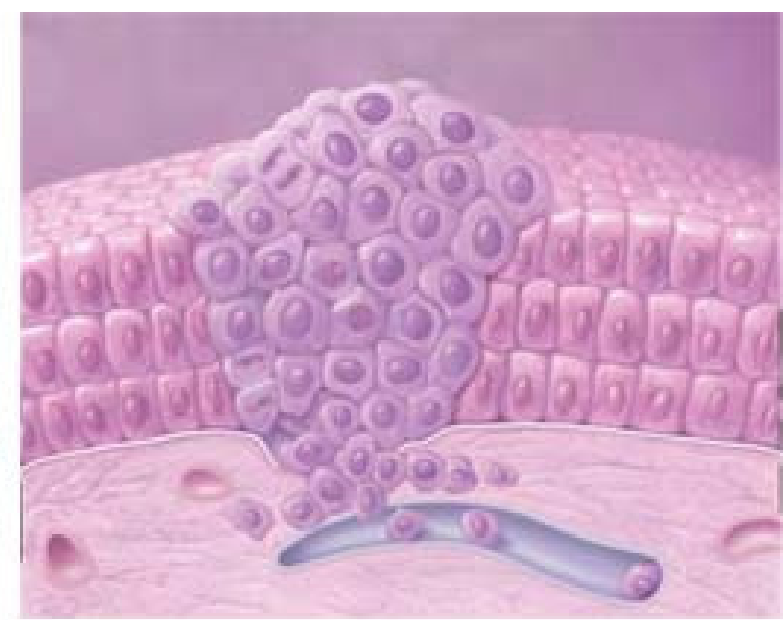

b)

Figure 1.3 a) Normal cells, b) Cancer cells [8].

Benign masses can be formed by either the unusual growth of the breast cells, or by the normal accumulations of fluids or cells inside the tissue. These developments in the breast do not exhibit metastasis. The development of the small milk ducts, lobules, and acini are regulated and dependent on the hormone level of the female subject. Changes of the epithelial layer may lead to the delay or the lack of hormonal related activities, which may cause some alterations in the breast tissue. This process is typically accompanied by the accumulation of some fluids like milk, mucin, or cells inside the cavity of the swollen lobule. The most common types of such benign tumors are the cysts [3], which may be detected with ultrasound imaging methods.

The types of benign and malignant breast masses are diverse. Common types of benign breast masses include fibrocystic diseases, fibroadenomas, and even abscess. Malignant breast mass types have many histopathologic forms that include; infiltrating ductal carcinoma (IDC), infiltrating lobular carcinoma (ILC), ductal carcinoma in situ (DCIS) and lobular carcinoma in situ (LCIS) [10]. The main breast mass types are listed in Table 1. 
Table 1.1 Main breast mass types [10].

\begin{tabular}{|c|c|c|}
\hline No. & Breast Mass & Type \\
\hline 1 & Fibroadenoma & Benign \\
\hline 2 & Fibrocystic changes & Benign \\
\hline 3 & Intraductal Papilloma & Benign \\
\hline 4 & Abscess & Benign \\
\hline 5 & $\begin{array}{l}\text { Infiltrating Ductal or } \\
\text { Lobular Carcinoma }\end{array}$ & Malignant \\
\hline 6 & $\begin{array}{l}\text { In situ Ductal or Lobular } \\
\text { Carcinoma }\end{array}$ & Malignant \\
\hline 7 & Inflammatory Carcinoma & Malignant \\
\hline
\end{tabular}

\subsection{Breast Tissue Biomechanics}

Many studies considered the breast tissue as primarily an elastic isotropic material, where its properties are independent of the applied strain rate. This was for the purpose of developing approximate mathematical models that can be used to estimate the tissue material properties [11]. In addition, the commonly used assumption was that the tissue is nearly incompressible, as the tissue's mass density exhibits an insignificant degree of change during deformation [12, 13]. This effect takes a place when the tissue is slowly deformed, and the local tissue density is not affected. During slow deformation, only the shape of the tissue is affected, and in this case the shear modulus is the main material property that characterizes tissue behavior. Using these assumptions, modeling the behavior of the breast tissue was possible by defining the elastic modulus or the shear modulus, using a simplified linear hook's law. Currently used ultrasound methods for breast tissue visualization and characterization are based on determining the elastic modulus or shear modulus; those methods are given the general term strain imaging techniques.

However, breast tissue as a biological material exhibits a nonlinear stress-strain relationship. This nonlinear behavior of the breast tissue can be explained by the presence of collagen networks and fibers that provide the tissue structure [14]. This nonlinear stress-strain curve has two regions. First, a linear region corresponding to the elastic tissue behavior and that part usually occurs at low 
strain values. The second region is a steep nonlinear one that occurs at higher strain values, which corresponds to the collagen network when its properties are taking effect. Usually exponential or power mathematical expressions are used to describe the steep region of the stress-strain curve.

In vitro mechanical measurements [11] using multiple indentation tests were performed on a number of different breast tissues and mass samples, to characterize their biomechanical behavior by plotting the stress-strain curve for each type, as shown in Fig. 1.4. This study used fresh samples that were excised directly from the patient in the operating room to the testing equipment to ensure accurate results and avoid tissue aging. The figure shows that there exists a broad behavior range for each type of tissue tested. Yet, the general behavior was linear in the initial part of the curve; which represents the linear elastic region, and then stress increases rapidly with increasing the strain, showing a nonlinearity degree that differs according to each tissue type.

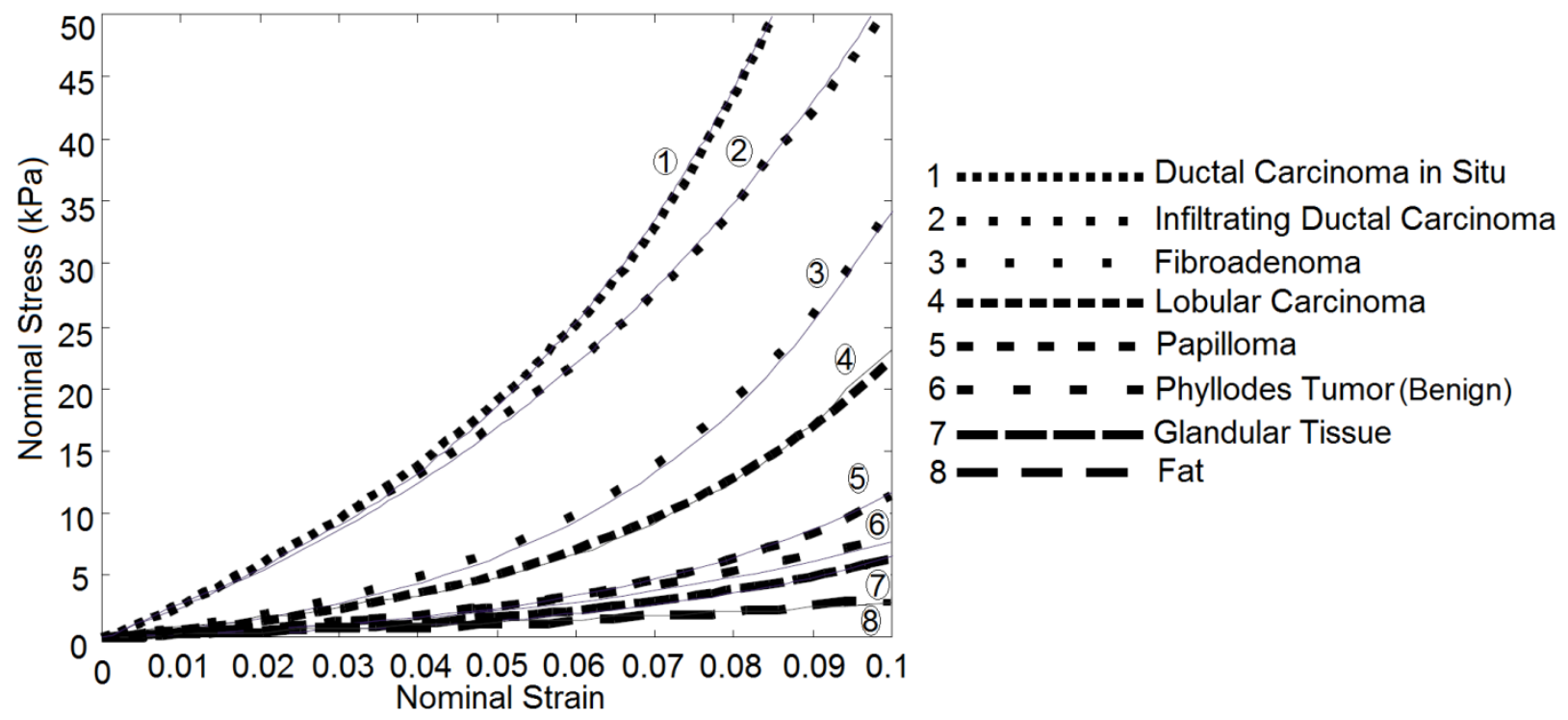

Figure 1.4 Measured stress-strain curves for eight different kinds of breast tissue [11].

Malignant tissues are not only stiffer than fat and the normal surrounding tissue, but also exhibit a high degree of nonlinearity [15-17]. It was observed that carcinomas are characterized by a large nonlinearity and high stiffness, while fat is particularly soft and nearly linear in behavior or quasi linear. Using an exponential fit on the measured data, the nonlinearity degree is reflected on the exponent's constant value and high stiffness. For cancerous tissues the exponent of the exponential fit was approximately twice that of normal glandular tissues and fat [11]. Other ex vivo 
studies agreed on this nonlinear behavior and used multi modulus measurements at various strain levels to examine this tissue nonlinearity $[18,19]$.

The differences in the nonlinear behavior of benign and malignant masses are thought of as an additional classifying feature between the main two breast mass categories. Beside the absolute stiffness of the tissue, the degree of nonlinearity; if measured, can serve as a new classification feature that can help in the prognosis of breast masses, before performing biopsy. Exploiting this property in a noninvasive way, would eliminate the unnecessary painful biopsies performed for the benign cases.

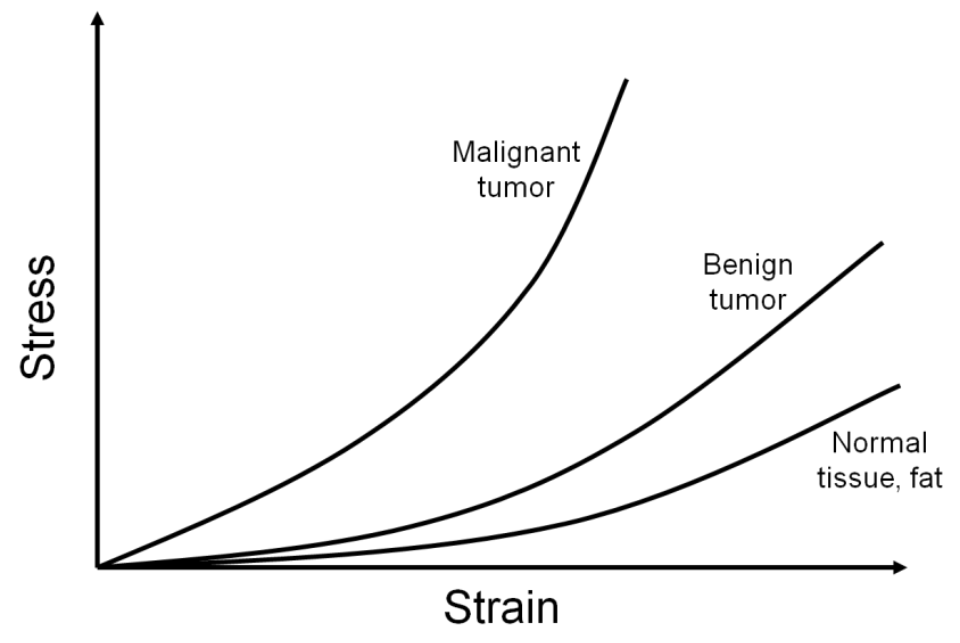

Figure 1.5 Breast tissues simplified biomechanical behavior.

Using a simplified biomechanical model, as shown in Fig. 1.5, the theory behind the nonlinear mass classification method becomes more profound. Normal tissues were modeled as having a mild nonlinear stress-strain curve; benign masses had a stronger nonlinearity while malignant masses had the steepest and strongest nonlinearity. This nonlinear stress-strain tissue property was exploited throughout our research to classify and characterize breast masses in vivo based on ultrasound elasticity imaging techniques. The detailed classification process will be described in chapter 3 .

\subsection{Breast Imaging}

The main breast imaging modalities that are currently in use, or have a potential for diagnosing breast masses are listed and compared in Table 1.2. A brief description of mammography, ultrasound imaging and ultrasound elastography are presented in subsequent sections. 
Magnetic resonance imaging (MRI) is currently not employed as a screening tool by itself, as it may overlook some tumors that conventional mammography can detect. MRI can be used to better examine suspicious areas found by mammography, and in women that have already been diagnosed with cancer to estimate the actual size of the tumor. Although MRI is generally more sensitive in detecting tumors than mammograms, false positive findings were observed, which means that the patient has to return for further examinations or biopsies [20]. In addition, a contrast agent might be needed to provide better imaging quality, which adds to the overall cost of the test [21]. MRI has also been associated with delays in cancer treatment and higher rates of mastectomy [22]. For these reasons MRI is not used as a screening tool for patients with average cancer risk, as further examinations in a large portion of the imaged subjects may be needed [21, 23]. Nevertheless, MRI is becoming increasingly in use for preoperatively evaluate DCIS tumors to define their extent [20, 24].

Breast computed tomography (CT) in general is very comparable to conventional mammography in detecting breast tumors, due to the common used technology in both modalities. However, CT offers higher contrast images with three dimensional capabilities, which adds to the imaging power of this tool, besides being more comfortable, as no tissue compression is needed. Nevertheless, mammography outperforms CT in visualizing microcalcifications [25]. In addition, breast CT suffers from the decreased sensitivity in diagnosing tumors within dense breasts. Dedicated breast $\mathrm{CT}$ equipments is still facing technological challenges, regarding the optimum imaging parameters, beam profile and the usage of contrast agents [26, 27].

Positron emission tomography (PET) is currently approved to monitor diagnosed breast cancerous tumors' response to treatment, but not as a screening or diagnosis tool [21]. Clinical usage of PET is limited to tracing metastatic cancer tumors that may travel from the breast tissue to other locations in the body. PET scans show relative accuracy in detecting large and aggressive tumors related to metastatic tumors, while it is less sensitive in locating smaller and less aggressive tumors (less than $8 \mathrm{~mm}$ ), due to the inherent low resolution PET images. The low sensitivity of PET imaging in detecting early stage breast cancers was reported, which do not support the practice of using this technique as a primary screening tool $[28,29]$. In addition, a nuclear radiotracer must be injected for PET imaging, which adds to the overall cost of the examination [21]. 
Table 1.2 Main imaging modalities comparison [21].

\begin{tabular}{|c|c|c|}
\hline $\begin{array}{l}\text { Imaging } \\
\text { Modality }\end{array}$ & Advantages & Disadvantages \\
\hline Mammography & $\begin{array}{l}\text { - High contrast. } \\
\text { - Good sensitivity and specificity rates. } \\
\text { - Used for routine screening. } \\
\text { - Ability to show microcalcifications. }\end{array}$ & $\begin{array}{l}\text { - Sensitivity declines with dense } \\
\text { breasts. } \\
\text { - Uses ionizing radiation. } \\
\text { - Requires tissue compression. }\end{array}$ \\
\hline $\begin{array}{l}\text { Conventional } \\
\text { Ultrasound }\end{array}$ & $\begin{array}{l}\text { - Does not use ionizing radiation. } \\
\text { - Particular use in distinguishing } \\
\text { between solid tumors and fluid-filled } \\
\text { cysts, } \\
\text { - Usage in biopsy guidance. } \\
\text { - Cost efficient. }\end{array}$ & $\begin{array}{l}\text { - Hard to diagnose malignant masses. } \\
\text { - Low sensitivity and specificity rates } \\
\text { when used alone. } \\
\text { - Operator's skill dependent. }\end{array}$ \\
\hline MRI & $\begin{array}{l}\text { - Does not use ionizing radiation. } \\
\text { - High contrast. } \\
\text { - Good sensitivity rate. } \\
\text { - Ability to show auxiliary nodes. } \\
\text { - 3D capabilities. }\end{array}$ & $\begin{array}{l}\text { - Cost inefficient. } \\
\text { - Some masses can be miss-detected. } \\
\text { - Low specificity rates. } \\
\text { - Not suitable for patients with metal } \\
\text { implants. } \\
\text { - Contrast dye might be needed for } \\
\text { some cases for imaging. }\end{array}$ \\
\hline $\begin{array}{l}\text { Ultrasound } \\
\text { Elastography }\end{array}$ & $\begin{array}{l}\text { - Does not use ionizing radiation. } \\
\text { - A number of quantitative parameters } \\
\text { exist for mass diagnosis. } \\
\text { - Good sensitivity and specificity rates. } \\
\text { - 3D capabilities. } \\
\text { - Characterize tissue nonlinearity and } \\
\text { biomechanics. }\end{array}$ & $\begin{array}{l}\text { - Lower contrast for benign cases. } \\
\text { - Requires tissue compression. }\end{array}$ \\
\hline PET & $\begin{array}{l}\text { - Provides functional and biochemical } \\
\text { information. } \\
\text { - High contrast. } \\
\text { - 3D capabilities. } \\
\text { - Sensitive to large metastatic tumors. }\end{array}$ & $\begin{array}{l}\text { - Uses ionizing radiation. } \\
\text { - Cost inefficient. } \\
\text { - Low resolution. } \\
\text { - Less sensitive to masses smaller } \\
\text { than } 8 \mathrm{~mm} \text {. }\end{array}$ \\
\hline Breast CT & $\begin{array}{l}\text { - High contrast. } \\
\text { - 3D capabilities. } \\
\text { - Better tumor margin visibility. } \\
\text { - Ability to show microcalcifications. }\end{array}$ & $\begin{array}{l}\text { - Uses ionizing radiation. } \\
\text { - Technical challenges still exist for a } \\
\text { commercial design [25]. }\end{array}$ \\
\hline
\end{tabular}




\subsubsection{Ultrasound Imaging}

Ultrasound imaging, sonography or B-mode imaging is currently a well established medical technology with diverse fields of applications. Ultrasound waves are the acoustic waves traveling with frequencies above the audible range of normal human hearing; which starts from about $20 \mathrm{kHz}$ up to few hundred's MHz. Medical ultrasound technologies normally use a fraction of the ultrasound spectrum; from 1 to $18 \mathrm{MHz}$, to combine both the good resolution and the wave penetrating ability [30]. The selection of the operating frequency is a tradeoff between the imaging spatial resolution and depth, where using lower frequencies generate less resolution but deeper image penetration into the body. Higher frequency ultrasound waves encompass smaller wavelengths and therefore are able to reflect or scatter from smaller structures. Higher frequency ultrasound waves exhibit a larger attenuation behavior, and consequently are absorbed more in the soft tissue, which limit their depth of penetration into the body; imaging depth [31]. Ultrasound imaging is valuable for imaging soft tissues. Superficial tissues such as breasts, eyes, muscles, tendons, ligaments, testes, and the neonates are normally being imaged at high frequencies ranging from 7 to $18 \mathrm{MHz}$. This provides an improved axial and lateral resolution down to sub-mm. Bodily deep structures such as heart, kidney and liver are imaged at lower frequencies ranging from 1 to 6 MHz. This low frequency range provides a decreased axial and lateral resolution, but deeper tissue penetration is obtained. Nowadays, echocardiography is used to almost any patient suffering chest pains. In many organs of the body, suspected tumors are scanned with ultrasound modalities routinely. This complements other main diagnostic imaging modalities, such as MRI, CT, PET and X-ray. Compared to almost all of these modalities, ultrasound systems are compact, lightweight, non-invasive and inexpensive. The main ultrasound scanner components are only a handheld ultrasound transducer and a processing and display console. In addition, ultrasound waves are nonionizing and cause very few or no side effects at all [31].

For human breast imaging ultrasound sonography is primarily used to determine whether a mass is cystic or solid. Moreover, ultrasound is used for biopsy guidance and also as an additional means to classify breast masses. Since a breast cyst contains a fluid component, its B-mode image has a well outlined mass, oval or round shaped, with a defined anterior and hypo echoic interior, This is in contrast to the malignant lesion's ultrasound images, which are not well outlined, with irregular shapes and have non uniform echoes. Another important classical ultrasound imaging feature differentiating between benign and malignant masses is the prominent shadow posterior to 
malignant masses. An explanation for this mass posterior shadow is that the absorption of ultrasound energy by the epithelial layer in malignant masses is usually higher than those observed in cystic masses or other types of benign masses [3].

Ultrasound imaging also has the benefit of being able to estimate some mechanical properties of the tissue under examination directly, while other imaging modalities must deduce these properties indirectly [32]. This is because tissue mechanical excitation is performed with the ultrasound probe itself, while for the other modalities, the excitations has to be performed using other devices than the sensing receptors. Imaging elastic quantities of the human tissue is generally referred to as elasticity imaging. Traditional medical imaging modalities calculate the spatial tissue deformations, and from which the distribution of the elasticity properties is reconstructed in the form of images. According to the imaging modality used, two main types of elastography imaging exist. The first type is ultrasound elastography (sometimes the term USE is used) and the second one is the magnetic resonance elastography (MRE) $[8,33]$.

\subsubsection{Elastography}

One of the major elasticity imaging methods is the ultrasound elastography. Elastography was proposed as a method for constructing tissue elastic modulus images quantitatively. Elastography were first addressed 1991 by Jonathan Ophir's group [34, 35]. The general principle of elastography is to apply displacement within the examined tissue by mechanical stimulation or compression. Then, the technique is utilized to calculate small fractional displacements between consecutive ultrasound image frames that are acquired and stored under compression. For ultrasound elastography, three methods exist for the induction of internal tissue strains: quasi-static, harmonic and transient elastography, Fig 1.6 [36].

In quasi-static elastography, a small displacement is induced within the tissue with a quasistatic mechanical source, such as the ultrasound transducer itself. The applied displacement is typically about 1 to $2 \%$ of the whole tissue axial dimension. The axial displacement component of the tissue under compression is then estimated. With this method, soft tissues are modeled as a one dimensional series of springs that are arranged in a sequential manner. 


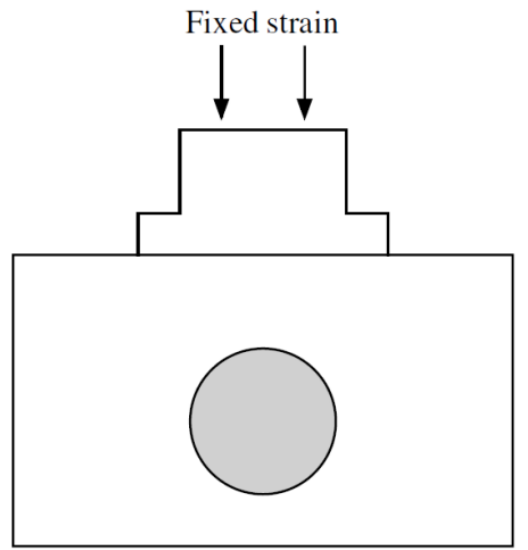

Quasi-static

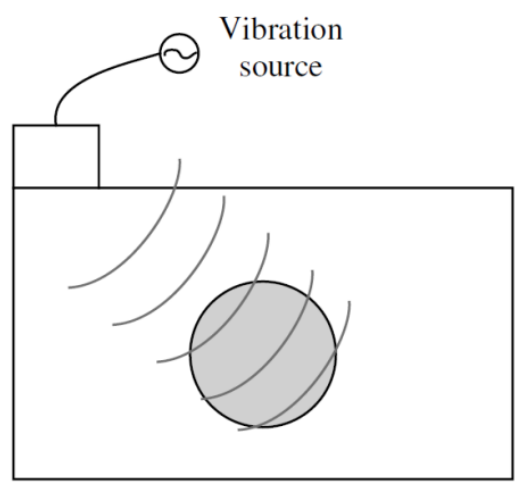

Harmonic

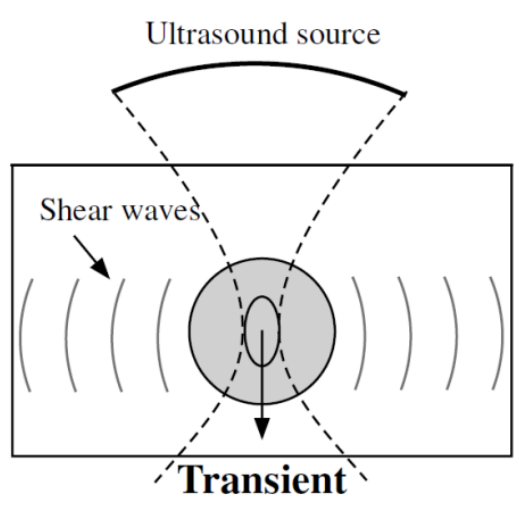

Figure 1.6 Schematic representation of current approaches to elastographic imaging [36].

In the method of harmonic elastography, low frequency acoustic waves, in the range of $1 \mathrm{kHz}$ or lower, are transmitted through the tissue with a mechanical source emitting sinusoidal waves. Using color Doppler imaging, the phase and amplitude of the propagating waves are visualized. One major limitation of this elastography method is that the induced shear waves attenuate quickly as they propagate through the soft tissue, which limits the wave penetration depth. This limitation was overcome using a transient approach to elastography. That approach uses a special ultrasound transducer that emits an acoustic radiation force (ARF) to perturb the tissue locally. In addition a custom ultrasound unit is needed to acquire images with ultra high frame rate of about 10,000 frames per second for the purpose of tracking the shear wave propagation, which is why this method is called shear wave elastography. Local shear modulus estimations of the tissue are usually made by calculating the shear wave speed directly from the arrival times [36]. Our study uses the quasi-static elastography approach to estimate tissue strains, because it requires no additional components other than the commercially available ultrasound units. We shall refer to it as elastography throughout the text.

Elastography has been applied in many applications for the diagnosis and characterization of many tissue types. Elastography has also been applied in vivo studies on muscles, prostate, vascular tissues and the breast. Additionally, elastography has demonstrated as being a potential tool to monitor thermal therapy. Generally speaking, cancer tissues are stiffer than the adjacent normal tissues due to their higher cell density than the surrounding normal tissue. This characteristic feature 
made elastography a powerful technique for cancer detection, as a quantitative stiffness contrast visualizing technique [15-17].

Elastography imaging has been studied extensively in the literature in many aspects, including the technique itself, image quality measures and mass classifications. The literature showed the potential of elastography as a stiffness quantification method for breast masses, which was previously judged by physical examination in a subjective way. Dual display mode visualization using both elastography and sonography showed the usefulness of this technique to characterize and classify masses as being either malignant or benign. It was observed on elastography that benign masses appear smaller on elastography than the corresponding sonography image, while malignant masses appear larger. This ratio of relative mass size on elastography to sonography was proposed as a classification criteria for benignity or malignancy [37]. Another proposed parameter was the stiff mass to soft tissue strain ratio, which reflects the relative stiffness between the suspected mass and the surrounding healthy tissue. This parameter was based on the fact that the fat biomechanical properties are rather constant over a wide range of applied stresses, whereas stiff breast masses have variable biomechanical properties, and under compression both types of tissue would have different strain values [37]. More details on the technique will be presented in chapters 2 and 3.

\subsection{Dissertation Objectives:}

Breast cancer caused what is equivalent to $14 \%$ of all female deaths due to cancer [38]. It is the second most common cancer among females in the United States, after lung cancer [39, 40]. Current diagnostic methods that are used for diagnosis of breast tumors have some disadvantages. Mammography imaging in general demonstrates a high sensitivity for the detection of breast cancer, despite being a two dimensional imaging modality. It has helped to increase the survival rate for breast cancer. However, in females with dense breasts, the sensitivity declines significantly and can turn down as low as $62 \%[41,42]$. Conventional ultrasound imaging modality have been suggested as additional methods for screening patients at risk of breast cancer when mammography is not a candidate method for them. Yet, it is hard to determine the type of the tumor from B-mode images alone. B-mode images underestimate the size of specific types of tumors. In addition, some tumors show strong shadows, which further degrade the produced image quality [3, 41]. Tissue biopsy is routinely performed under conventional ultrasound guidance, although four out of five biopsies yield a benign result [43]. Biopsy is also considered the most costly part of breast cancer 
examination and its invasive nature is another disadvantage [43, 44]. Furthermore, breast cancer can unexpectedly travel to other healthy organs because of metastasis. This makes the early detection of breast cancer a crucial task to obtain an accurate prognosis and diminish the increasingly risk of breast cancer.

The main objective of this dissertation is to investigate a new method to accurately diagnose breast tumors, and reduce unnecessary biopsies. The specific objectives of this research can be summarized as:

1. Visualize stiff masses using strain images in $2 \mathrm{D}$ and volumes in $3 \mathrm{D}$ for both breast phantoms and human subjects. 3D imaging would provide more information about the morphology and size of the targeted breast mass, and facilitate diagnosis.

2. Classify breast masses based on a nonlinear model. A new classification method is proposed and applied. The new method would provide an improved discrimination feature between benign and malignant masses.

3. New strain imaging types are proposed and constructed in $2 \mathrm{D}$ and 3D. The new types would provide better discrimination for the stiff mass from the surrounding soft tissue.

4. Delineate the suspected breast masses using active contour segmentation algorithms. This would provide a reliable tool for measuring the volume of the breast mass as well as an enhanced visualization of its outline in 3D.

Following these specific aims and achieving them, the final goal of visualizing and classifying breast masses would be obtainable and feasible. Long-term objectives are to provide clinicians and scientists an accurate, portable and fast diagnostic tool for a large clinical translational research in rural areas. As the results will show, the impact of this research on breast care would be significant regarding the time and cost of diagnosis and also the comfortability of patient's diagnosis.

\subsection{Innovation and Significance:}

The work presented in this dissertation has many significant contributions in the field of breast cancer visualization, detection and classification. These concepts can be easily translated to other types of cancers such as cervical, prostate, pancreatic, liver and ovarian cancers. New ideas are suggested and implemented to obtain new forms of human breast imaging modalities using ultrasound elastography. In addition, a new quantitative mass classification method is proposed and tested in a pilot in vivo study. The novel key points in this research can be summarized as follows: 
1. A modified strain imaging algorithm: The algorithm used for constructing the strain images was based on a one dimensional tracking of temporally windowed signals. The new key features of the used algorithm were using direct signal interpolation before cross-correlation, and using spline smoothing function on the estimated displacements. Both operations provided an enhanced image quality and reduced the effects of random noises.

2. In vivo $3 \mathrm{D}$ strain imaging: $3 \mathrm{D}$ imaging is advantageous over $2 \mathrm{D}$ imaging as it convey information about the tissue in the elevational dimension as well as the axial and lateral dimensions. It facilitates better visualization of the region of interest and more accurate estimation of the shape and size. According to our best knowledge, this is the first time 3D in vivo elastography imaging is presented for human subjects. This imaging modality has not been reported before except for breast phantoms. Additionally, the usage of 3D sector ultrasound probes is also novel in this study and has not been demonstrated in the literature. Using sector probes incorporate more complex and costly image processing algorithms, and the verification of the methods using sector probes ensure that the conventional linear probes would also work as well.

3. Novel classification approach based on material nonlinearity: Currently most of the classification methods are based on a linear model. The proposed mass classification method is based on a nonlinear model of the tissue under investigation. The estimation of the strain differences between the breast mass and the surrounding soft tissue were observed over different pre-compression levels. The parameter describing the nonlinear (or linear) behavior of the tissue was utilized in the characterization process. The estimated nonlinear parameters were calculated, assuming that the breast mass's material is similar to a powerlaw material, like rubber. This is the first time such a model is exploited in breast masses characterization. Linear and logarithmic relationships were also considered, and shown to be effective in the mass classification process. The discrimination margin between the main two mass types was found to be larger using the proposed approach.

4. New strain types imaging: Derived strains were exploited in constructing new elastographic imaging types. They would provide a visual improvement to breast stiff mass imaging. The derived strains show the breast mass and the mass-background border with different degrees of clarity. The derived strain used were the first principal, Von Mises and maximum shear strains, with the latter providing the highest degree of boundary enhancement. Automatic 
segmentation techniques based on active contour methods can be optimized using the maximum shear elastography. Those types of segmentation algorithms have the limitation of requiring the masses to be relatively regular, well-circumscribed single margins on the images, which is not the case with breast masses. This limitation can be overcome by using the new derived elastography strains, as the boundaries become more profound in the resulting images and volumes.

5. Application of active contour segmentation algorithms: After classifying the breast mass as either benign or malignant, the shape and extent of the mass should be assessed for better and more accurate prognosis. The purpose of the segmentation is to delineate the stiff breast mass from the surrounding soft and healthy tissue. Construction of elastography images and volumes showing only the isolated mass would provide a more accurate description of the mass's shape, and also provide a reliable tool to determine the size of the mass. The use of a geometric active contour model for segmenting breast masses, based on the constructed 3D elasticity volumes, has not been reported before, according to our best knowledge. Additionally, the new derived strains provided a degree of mass's boundary enhancement, which ultimately improved the performance of the segmentation performance, by placing the outlining contours at the required locations.

Our vision regarding the new methods proposed in this dissertation is making breast mass examinations more time and cost efficient. Mass localization using mammography and B-mode ultrasound imaging would be followed by mass characterization using elastography in the same session. The prior knowledge of the tumors type, shape and size preoperatively, would provide very helpful information about the extent of the disease, which ultimately helps to decide the sort and the level of treatment. The new methods would also minimize the number of unnecessary painful breast mass biopsies, which are the current routine procedures performed.

\subsection{Dissertation Outline:}

The rest of the dissertation is arranged as follows. Chapter 2 represents the background for this research. Literature review on ultrasound elastography, the technique description, various methods of estimations and the estimated strains types are presented. Three dimensional (3D) elastography is described along with implementations from previous studies. Breast mass classification methods are enumerated using linear and nonlinear biomechanical models. Different strain types that can be 
used also for elastography are presented at the end of chapter 2. Chapter 3 establishes the thorough framework of the materials and methods for this work. The method for ultrasound signal processing is demonstrated along with details on elastography images and volumes construction methods. In lab experiments (in vitro) on a tissue mimicking phantom are described and explained. In addition, an experimental clinical setup is illustrated and studies on human volunteers along with the new breast mass classification method are depicted. Description of the used segmentation algorithms is demonstrated, and the method of application on the in vitro and in vivo volumetric data is elucidated. Chapter 4 elaborates the in vitro results with 3D elastographic volumes showing stiff masses inside a soft phantom. New derived strain elastography is demonstrated in 2D and 3D. Image quality assessment is presented in the chapter and also mass volume calculations for all types of derived strains. Chapter 5 extends the lab experiments to clinical studies on human patients, and detailed results are discussed in the chapter. 2D and 3D elastographic imaging results for real patients are presented in the chapter. Also, the new breast mass classification method is applied on all patients involved in the study, and the classification is verified with biopsy diagnosis. The proposed classification method is compared with other commonly used methods regarding the performance of classification and underlying model. Additionally, the newly reported derived strain elastographic volumes are demonstrated, and their use in mass visualization enhancement is presented. Chapter 6 presents the application of two active segmentation algorithms. The best performing algorithm is selected for further analysis, based on the quality of the produced in vitro data, and the chosen method is then applied on the in vivo volumetric data. The effect of the derived strains on the segmentation performance is exhibited in the chapter. Finally, chapter 7 provides the general study conclusions and the impact of the presented methods on breast cancer detection is emphasized along with the proposed future work. 


\section{CHAPTER 2}

\section{LITERATURE REVIEW}

The topic of ultrasound elastography has been a hot subject in the literature for over twenty years. Many research and technical papers exist covering the theory, methods, clinical applications and validation of elastographic imaging techniques for both tissue mimicking phantoms, and live tissue. The diversity of publications available, made summarizing them a hard task. Nevertheless, this chapter reviews the literature for the most pertaining subjects that provide the background related to the work presented in this dissertation.

\subsection{Ultrasound Elastography}

\subsubsection{Background}

Elastography or ultrasound strain imaging is an emerging powerful modality for visualizing and differentiating between soft and stiff tissues in relatively homogeneous tissues [45, 46]. This modality offers an insight into the biological tissues elastic properties by calculating internal tissue displacements and hence estimating tissue strains. Because of inherent tissue heterogeneity, a considerable dynamic range of strains is expected to be estimated and visualized, wherein higher stiffness tissues would generally exhibit lower strain values than tissues with lower stiffness. This visualization of the tissue stiffness contrast is considered a key feature for cancer diagnosis [47]. Elastography was first addressed by Ophir et al. [34, 35]. The imaging methods and signal processing were introduced and applied on tissue mimicking phantoms, and later on in vivo human breasts. Basically, the examined tissue is compressed with a quasi-static compressor, such as the ultrasound probe itself. Radio frequency (RF) signals are acquired at the pre- and post-compression stages to form image pairs. Cross correlation operation is then applied between the pre- and postcompression frames to estimate the internal tissue displacements, which are in turn used to estimate tissue strains, through the gradient operator, and produce an elastogram; the final strain image. The main idea behind the technique is illustrated in Fig 2.1. 


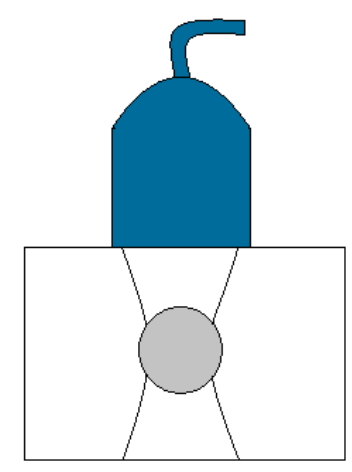

Pre-Compression

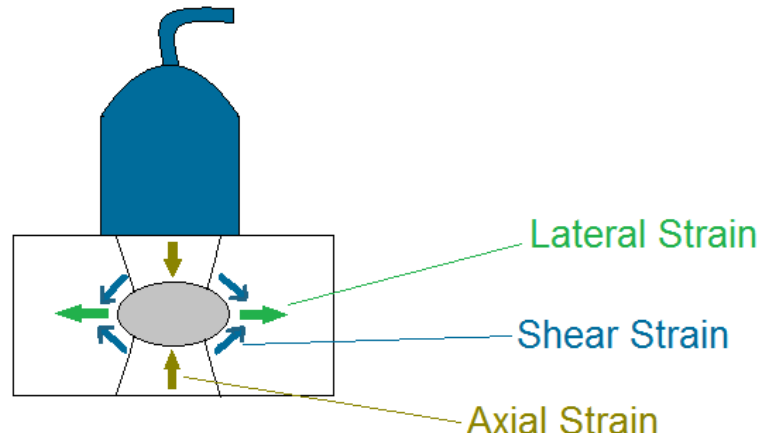

Post-Compression

a)

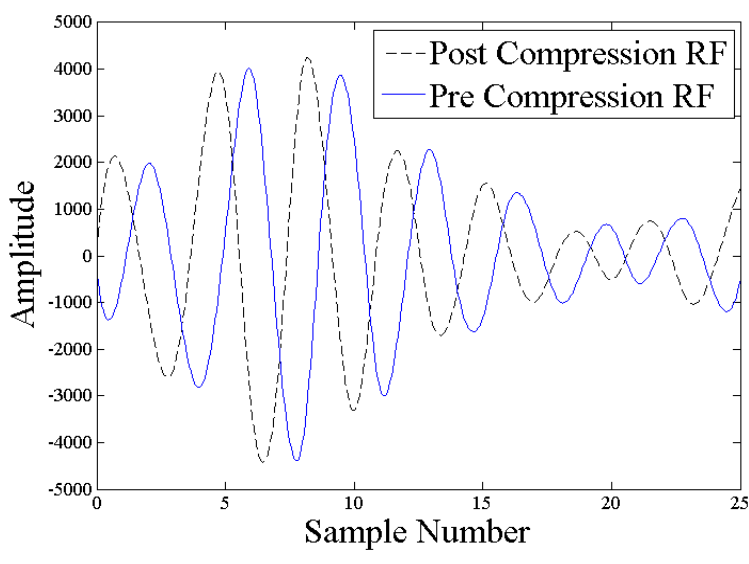

b)

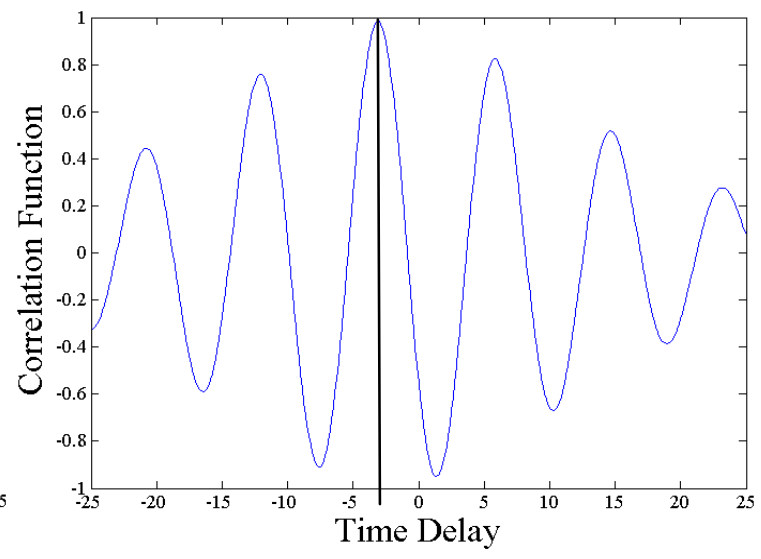

c)

Figure 2.1 Elastography technique: a) Tissue compression. b) Acquisition of pre and post compression signals. c) Cross-correlation function.

During the first decade of elastography development as an imaging modality (1991-2000), several basic issues were focused on. Those issues would define and support the medical and scientific viability of the technique. This was important in this early stage of research in order to gain comfortability with the technical prospects of elastography, and its potential in the biomedical applications. The issues that were considered during that period of time were: whether the technique works in simulations and laboratory studies at all, whether the technique works for in vivo cases and yield promising results, and "what are the theoretical mechanical, acoustic and signal processing 
parameters that impose limits on the maximum attainable elastographic image quality and what are their tradeoffs?" [48].

During the second decade of elastography development (2000-2010) research continued on some of the issues that were raised in the first decade. This facilitated defining all the important imaging parameters and their tradeoffs. The main parameters included signal to noise ratio, contrast-to-noise ratio, image resolution, and optimization via applying the proper range of strain levels, sampling rates and signal processing parameters selections. Additionally, the work started in deploying different strain components extended to result in two new variants of elastography. The first one was named poro-elastography. It involved imaging of the Poisson's ratio. Poroelastograms and their achievable theoretical image quality have been studied and results demonstrated its viability in imaging time dependent compressibility for both phantoms and live tissues. The second elastography variant, termed axial shear strain elastography was implemented and examined in simulations, phantoms and also in vivo breast masses [48-51]. Our work here incorporates using the axial shear elastography in strain imaging, as will be shown later. While poro-elastography was not exploited, and it was mention here for the review completeness.

\subsubsection{Signal Processing Methods}

In general, signal processing involves two main processes; time delay estimation and strain estimation. Each estimation method has its own advantages and disadvantage, which are listed in this subsection. Different approaches have been described in the literature [35, 47, 48, 52-57] to estimate time delays between the corresponding RF lines. All of them are based on measuring precompression and post-compression signals similarity. Some of the similarity measurement methods, already available in the literature of elastography are summarized below:

1. Cross-correlation: Normalized cross correlation of temporally windowed pre- and postcompression signals is calculated for a predetermined range of time delays. The time delay estimate is calculated at the maximum value of the correlation function as the subsample signal delay. The cross-correlation function is interpolated to obtain a better resolution estimates for the subsample delays [35]. This method is the one used in our research with some modifications.

2. Direct signal level comparison: Direct comparisons of pre- and post-compression signals zero crossing or level crossing provide the displacement estimate. This method has some 
benefits of being a real time method with good resolution and also simple. However it has disadvantages regarding the accuracy of displacement estimation being lower than window based methods, and low robustness of the method for large displacements [58].

3. Sum of absolute difference (SAD): The SAD of temporal windows of pre- and postcompression signals is calculated for a number of time delays of the post-compression signal. Subsample time delay estimate, or an integer of it is attained as the minimum value of the SAD function. SAD displacement estimators typically suffer from lower signal to noise ratio than the cross-correlation methods [59].

4. Multi resolution displacement estimation: Displacement estimation is performed using windowed signal segments with varying lengths to provide displacement estimation with multi resolutions [60].

5. Lateral displacement estimation: This type of methods are typically estimated using the traditional cross correlation method, but in this case the method is applied on two dimensional windows of RF signals, either interpolated or non-interpolated. Lateral displacement estimation can also be made with the tracking of one dimensional windows in the lateral direction [61].

On the other hand, strain estimation methods are based on gradient estimation, direct slope approaches, or hybrid estimation methods [48] as briefly summarized below:

1. Gradient based strain estimation: Calculation of strain is performed by finding the local gradient of the estimated displacements. The signal to noise ratio of such a method depends on the distance between the displacement estimates used for calculating the gradient. Gradients are very sensitive to noise, so low-pass filtering of the displacement estimates is a necessary step and it is often performed before computing gradients. Low-pass filtering methods include median filtering, but are not limited to such filtering methods [62]. This method is the one used in our research.

2. Direct slope estimation: The slope of the displacement with respect to the depth function can be used as a direct estimate for strain. Typically least square methods are used for curve fitting. This method has the advantage of a high signal to noise ratio. A disadvantage of it is the poor spatial resolution. This is because of the need to a relatively large number of samples to improve the fit robustness [63]. 
3. Direct strain estimation: This method exploits stretching the post-compression signal by a number of factors until the cross correlation coefficient reaches a maximum value. With this approach the strain approximately equals one minus the iteratively obtained stretch factor. That strain is thus directly estimated as it corresponds to the maximum correlation coefficient. The method has the advantage of being robust to noise. Disadvantages of the method include being costly in the number of computations needed, and a questionable strain estimation accuracy [64].

\subsubsection{Elastography Imaging Artifacts}

Like any other imaging modality, elastography has a number of imaging artifacts that have been described in the literature. Some of these artifacts appear when the method is suboptimal, and many of them contain diagnostic information [37].

1. Stress/strain concentration artifact: The presence of a stiff or soft mass with sharp edges brings concentrations of stresses and strains around the mass. These are areas marked with low strain values and may be mistakenly interpreted as stiff tissues [19]. This artifact in general is a common one in elasticity imaging, and because of it, medical practitioners require training on how to distinguish the real masses from the false ones created by low strain areas.

2. Sliding artifact: When a bright ring or group of waves appear around a tumor on the elastogram, it indicates that while the elastogram was obtained, the tumor was moving out of the imaging plane. This artifact can be corrected by making the stiff mass stay in the imaging plane. This can be done by repositioning the patient, applying less compression, or letting the patient hold his/her breath. This artifact could be a sign that the tumor is moving freely within the tissue, and is therefore most likely, but not necessarily, benign [37].

3. If substantial compression was used when obtaining the images, bright and dark spots patterns are noted, representing noise. No useful clinical information can be provided with images with this kind of artifact. This artifact can be reduced by using the minimal compression necessary to achieve a good imaging quality [19, 37].

4. Bull's eye artifact: This unique artifact is noticed within cystic lesions. It appears because the fluid inside the cyst is moving, and decorrelation between images may occur. Although only a limited number of cases has been reported, this artifact was not observed in some 
types of cancer, like mucinous cancers. Clinical trials are still in progress to further study this property [37].

\subsection{D Imaging}

\subsubsection{D Elastography}

Three dimensional (3D) elastographic imaging is more informative as it presents better qualitative and quantitative measures of the size and shape of the cancer lesion. It is considered advantageous over the standard 2D strain imaging as it provides information on the extent of the lesion in the third dimension. It has the potential of identifying the actual shape of the stiff tissue, which would be an additional factor in tissue characterization.

Three dimensional elastography has been recently addressed by a number of authors. The first study that demonstrated elastographic imaging in 3D was done by Bilgen et al.[65]. They acquired acoustic data for mice from contiguous sections separated by $3 \mathrm{~mm}$, spanning the tumor in a number of planes. The 2D images were combined together after manual segmentation to form a $3 \mathrm{D}$ elastogram of the tumor. The 3D construction was very basic and the volume's slices were not consistent with each other, yet their work was the start of the research on volumetric elastography imaging. Patil et al. [52] reported 3D elastograms constructed by using a multistage strain estimation algorithm for a prostate phantom. RF data were acquired using a mechanically rotated linear ultrasound array operating at $12 \mathrm{MHz}$ frequency. Only the inclusions were constructed in $3 \mathrm{D}$ by using a segmentation algorithm to isolate the inclusions from the slices and the segmented shapes were rendered to reconstruct the 3D shapes of the inclusions, as shown in Fig. 2.2 a) [52]. No quantitative analysis was made by the authors. They contended that the 3D elastography has the potential to assist in discriminating benign lesions from malignant cancers by identifying the detected lesions on the basis of 3D shape in the elastograms.

Bharat et al. [66] presented 3D elastographic volumes for a tissue mimicking phantom of a canine liver with created thermal lesion in vitro [66]. A mechanically rocked ultrasound 3D curvilinear array probe operating at $4.4 \mathrm{MHz}$ was used for volumetric RF data acquisition. Manual segmentation was used to isolate the lesions from the elastograms and construct the lesions shapes in 3D after applying interpolation between subsequent segmented slices; Fig $2.2 \mathrm{~b}$ ). The constructed elastograms were used to calculate the volumes of the lesions for evaluation of accuracy. They concluded that volume calculations were underestimating the actual volume of the lesion. 
Another 3D axial strain map was developed by Deprez et al. [67] for a breast phantom and a pressure ulcer-mimicking phantom using an iteratively constrained optimization process to estimate the axial stains; Fig $2.2 \mathrm{c}$ ). However, their study did not include quantitative assessments. A drawback of the constructed volume was the low contrast between high and low stiffness regions in the phantom.

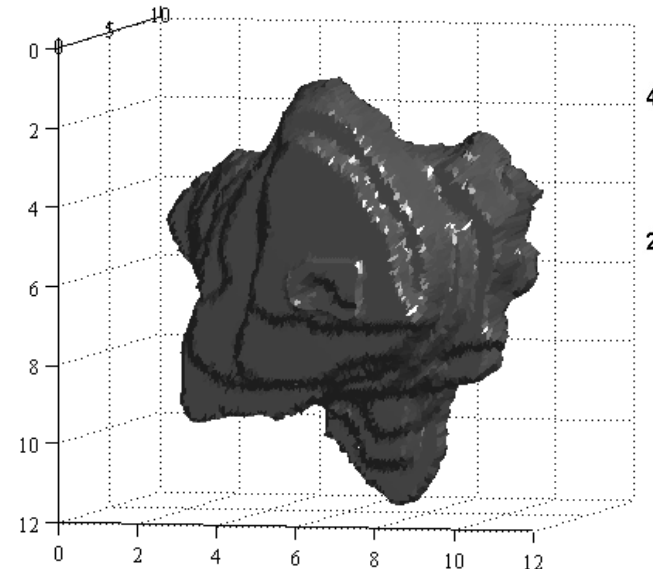

a)

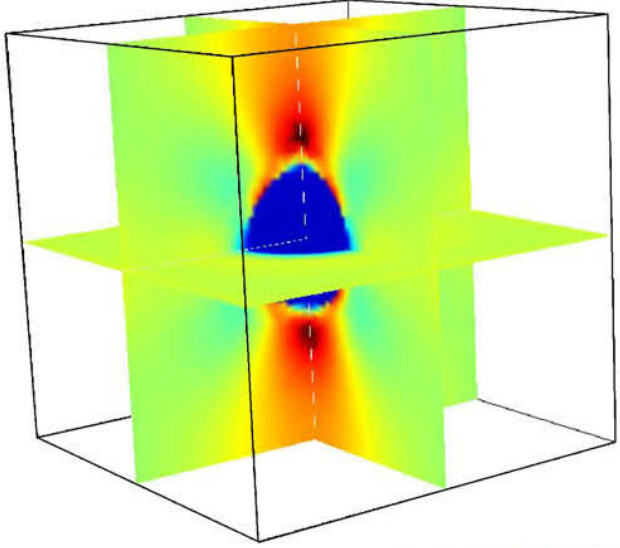

c)

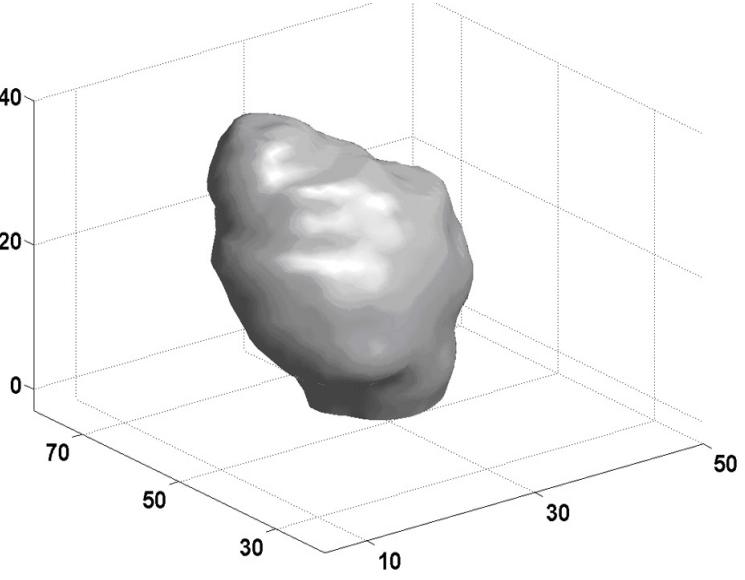

b)

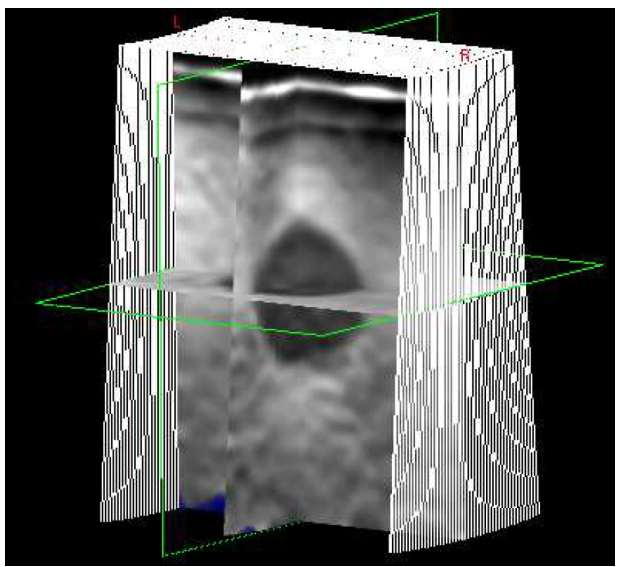

d)

Figure 2.2 Examples of 3D elastography in the literature: a) Patil et al. 2007 [52]. b) Bharat et al. 2008 [66]. c) Deprez et al. 2009 [67]. d) Housden et al. 2010 [46].

Another study used freehand ultrasound elastography with a 3D mechanically swept probe using 3D temporal windows for displacement estimation was presented by Treece et al [68] and Housden et al. [46]. Near real-time 3D elastograms were constructed for a number of phantoms, including agar phantom and a breast biopsy phantom; Fig 2.2 d). No quantitative parameters or 
image quality measures were performed in this study. Their main conclusion was that the $3 \mathrm{D}$ probe provided higher quality axial strain data, while the freehand approach is preferable in situations where limited access to the scanning target restricts the use of a large footprint probe.

A finite-element based quantitative 3D elasticity imaging algorithm applied on tissue mimicking phantom were reported by Richards et al. [69] using a 3D displacement estimation method. 3D slices of elastic modulus maps were constructed for a gelatin phantom with stiff inclusions. Quantitative measures were calculated for the relative strain and the volume of the inclusions. Volume estimates exhibited a small error, while the estimated relative strains suffered from a large error with respect to the mechanical measurements using the phantom. Only finite element implementation was done with no actual elastography imaging performed.

In conclusion, most of the previous studies on volumetric elastography did not show a complete region with the stiff lesion inside it, but merely segmented slices were staggered together to show only the stiff tissue, or displaying three planes of the region of interest. The studies were concerned mainly with phantoms and in vitro samples. We will show that our method can demonstrate a complete region of interest exhibiting both soft and stiff tissues together for both phantom and in vivo live tissue.

\subsubsection{Segmentation methods}

Image segmentation is the process of dividing the elastogram into a number of segments, to improve the appearance of the target breast mass and make it easier to analyze, which provides a reliable tool to determine the shape and size of the mass by estimating its volume. Manual segmentation is performed by tracing the targets boundaries, based on specified target features that is distinct from the rest of the image, such as color and intensity features. Manual segmentation is time consuming and subject to operator variations, therefore segmentation algorithms are implemented to avoid these limitations. Active contour segmentation methods are now the primary used automatic/semi-automatic segmentation algorithms in medical imaging [70].

Active contour models; namely snakes, are curves or contours embedded in the image domain. Those curves can move and deform under the influence of external and internal forces. External forces originate from the image topological data, while internal forces take place within the curve itself, due to its curvature and shape. 
Active contour segmentation algorithms are generally categorized as:

1. Geometric models (Level set methods) [71]: The dynamic contour is embedded as the zero level set, of a higher dimensional level set function that depends on time, where time reflects the evolving function deformation.

2. Parametric models (snake methods) [72]: in which the dynamic contour is defined by a spatial parameter. That parameter determines the location of each point in the evolving contour.

Both models have been applied in the segmentation of various medical imaging modalities, including CT, MRI and US images, with the growing interest of producing three dimensional segmentations [73-76].

Literature related to the application of active contour segmentation algorithms on ultrasound elastography images is very limited. One study by Liu et al [77] applied a parametric segmentation algorithm for RF ablated thermal lesions on 3D elastographic data; Fig 2.3. The authors concluded that this algorithm is efficient for segmenting lesions with regular and well-circumscribed single margins on the images, which is not always the situation with many in vivo elasticity images. To overcome this difficulty, a level set based segmentation technique was employed in our study.

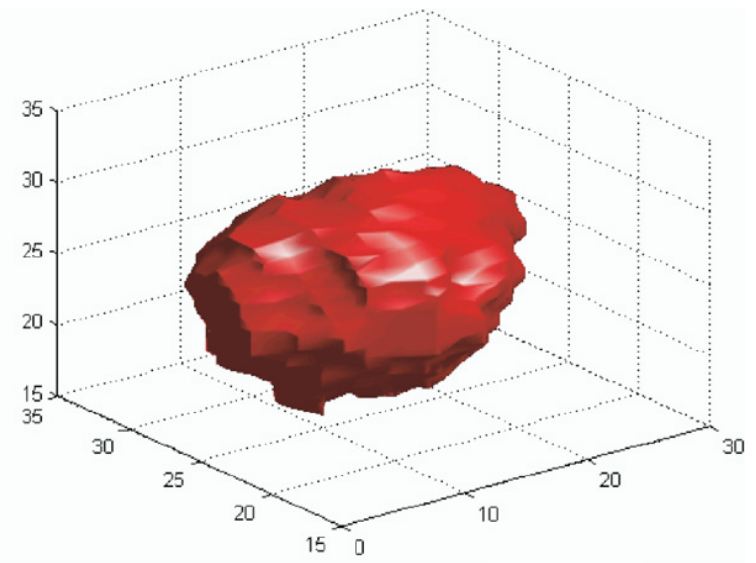

Figure 2.3 Application of a segmentation algorithm on 3D elastography data [77].

Capturing image's shapes and edges using the level set method was first introduced by Osher and Sethian [71]. The main advantage of this method is the ability to handle self-intersecting contours and sharp changes in topology. This segmentation method has been applied mainly for segmentation of tumors from B-mode ultrasound images [75, 78]. Only one study reported the 
application of level set segmentation algorithm [79] on elastography. Yet this study was limited to segmentation of 2D elastography images only, and the efficiency of the segmentation was very poor. As will be shown throughout the dissertation, the level set method is applied on 3D elastography data sets, both in vitro and in vivo, which, according to my best knowledge, has not been reported before.

\subsection{Tissue Classification using Elastography}

Modeling mechanical properties of biological tissues presents a challenge, since many of them are behaving as nonlinear, viscoelastic and sometimes anisotropic tissues [12]. We can realize that in a global sense, tissue does not hold to the definition of elastic materials, that requires a one to one relationship between the stresses and strains. To utilize elastography as a potential clinical tool for quantifying soft tissue's mechanical properties, a simplification to the modeling framework can be developed. Although soft tissues have a typical nonlinear response when investigating them for a large range of deformations, experimental studies for human tissues have shown [18] that the stressstrain relationship does approximately exhibit a linear behavior when the deformation is small (strains less than about $10 \%$ ). These study results mean that the effects of nonlinearity in relatively homogeneous tissue can be negligible and would be omitted for in the case of application of small strains. This was the basis for using the direct elastography methods in the linear region of the stress-strain relationship; subsection 2.3.1.

However, as tissues are heterogeneous in nature, the local strain distribution depends largely on the applied strain. It is important to notice that the material elastic modulus can be computed mathematically as the derivative of the stress-strain relationship curve. As a result, strain measurements would depend on the applied stress and therefore, the tissue elastic properties may be better defined if they were specified at an applied stress with known level. Tissue samples that exhibit nonlinear elasticity have a stress-strain relationship that mostly follows an exponential function or a close approximation to it [18, 80]. As discussed by (Fung, 1993) [12], this situation introduces an additional complexity, especially when trying to quantify and correlate the results of the clinical elastography studies. The nonlinear model bases elastography classification is presented in subsection 2.3.2. 


\subsubsection{Linear Mass Classification}

Assuming a linear biomechanical model for the breast tissue, a number of parameters were considered to classify breast masses, based on direct measurement from the elastography/B-mode images. Direct elastography classification of stiff masses being benign or malignant primarily depends on two linear parameters, as described from the literature [32, 37, 81-84]. The first parameter is the relative size of the mass imaged with both elastography and B-mode ultrasound; Fig 2.4 [32, 37]. If the mass was bigger in elastography images than B-mode images, this indicates malignancy. If the mass was smaller or equal to its size in elastography, then this indicates benignity. This phenomenon was explained by the desmoplasia that accompanies cancer [48]. Mass size measurement is done by either length or area. The sensitivity of using such a method is quite high, according to previous studies [32, 84, 85]. A drawback of this method of interpretation is that it requires the mass to be visualized well enough in the B-mode image, in order to obtain an accurate length/area measurement on both B-mode and the elastogram images. This condition is not always fulfilled for some types of malignant masses, like ductal carcinoma, and the overall performance of this method declines [37].

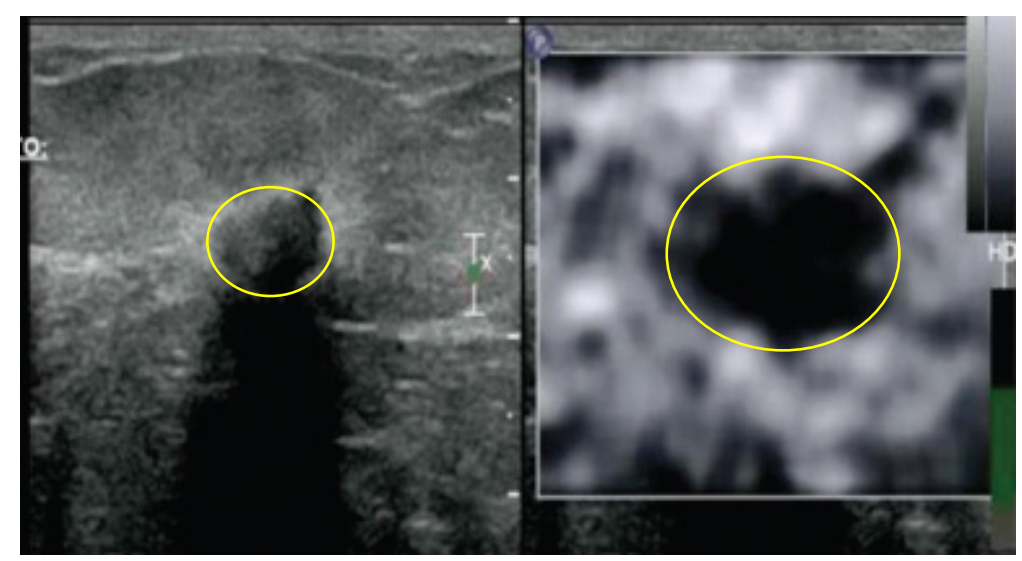

Figure 2.4 B-mode image and elastogram of cancer [37].

The second parameter is the relative stiffness of the mass with respect to the surrounding soft tissue. As mentioned earlier, this parameter uses the fact that malignant masses are stiffer than benign ones $[11,19,37,80]$. Typically the ratio is calculated by dividing the estimated strain values within the soft tissues by the values within the stiff mass. This ratio relied on the knowledge that soft tissue's properties or fat properties are nearly constant. On the other hand, the stiff mass's properties are considered to be variable, according to the examined mass type. Thus, masses with 
densities and stiffness similar to fat have small ratio values. Previous clinical studies suggested a fat/mass strain ratio of less than 4.8 as benign and a ratio of greater than 4.8 as malignant [85]. Automated measurements and ratios are available in some commercial units, but this was not FDA approved yet for use in the United States [37]. One difficulty with this parameter is that an overlap exists in the relative stiffness values between benign and malignant cases, and in some cases it is hard to diagnose the mass based on this parameter.

Breast elasticity images can be obtained in either a sitting position or supine position. The first position slightly limits the number of accessible masses for elastographic imaging, while the supine position allow the imaging of masses that are close to the chest wall. An example of elastograms and corresponding B-mode images of a fibroadenoma (benign tumor) and a carcinoma (cancer) is shown in Fig 2.5. It is clear the size discrepancy between the B-mode image and the elastographic appearance of the carcinoma unlike in the case of the fibroadenomas [19].

Fibroadenoma
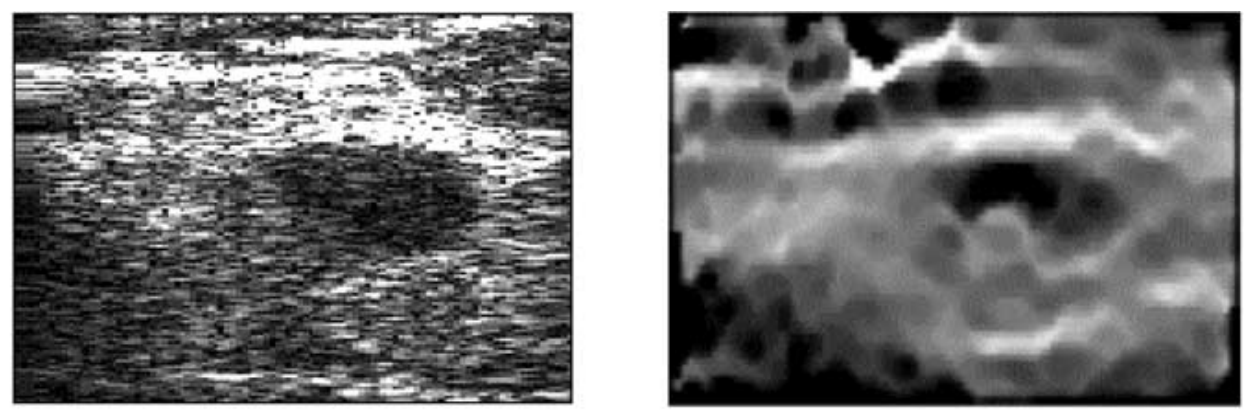

Infiltrating Ductal Carcinoma

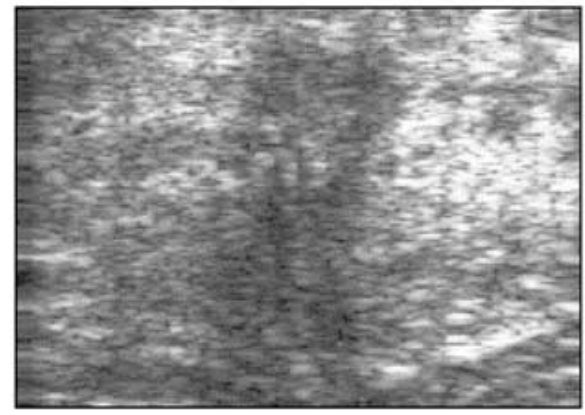

Sonograms

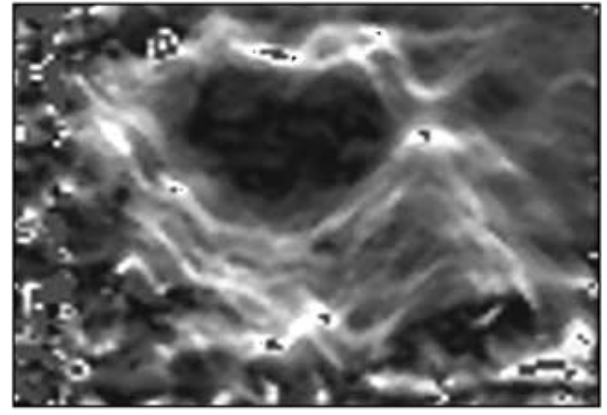

Elastograms

Figure 2.5 Breast cancer imaging using elastography: Sonogram and elastogram pairs from fibroadenoma and infiltrating ductal carcinoma [19]. 


\subsubsection{Nonlinear Mass Classification}

Several studies applied a nonlinear model for characterizing human tissues. Emelianov et al. [86] used elasticity imaging for estimating nonlinear tissue elasticity in vitro using a canine kidney and an agar phantom. They observed the strain values for the target stiff tissue over a range of deformation levels, and used the strain values to estimate a nonlinear parameter using interpolation and nonlinear curve fitting. Xu et al. [87] reported a similar method to characterize the edematous tissue in rats and to make a distinction between fibroses and inflammation in vivo. The developed strain inside a targeted lesion over a relatively wide dynamic range of the applied strain was recorded and plotted against each other. The estimated strain versus the applied strain curve was plotted and fitted using a nonlinear curve fitting equation. The exponent of the equation was considered to be a nonlinear charactering parameter, which successfully discriminated fibroses from inflammation within the participated mice.

However, few recent studies applied a nonlinear classification model for breast masses using elastography. Using mechanical measurements in vitro, an exponential stress-strain relationship was measured for a number of breast tissues, including fat, glandular tissues, fibrous tissues, and malignant masses; ductal carcinoma and invasive ductal carcinoma [11, 18, 19, 88]. In general, malignant masses exhibit a stronger nonlinearity than that observed for benign masses and the surrounding healthy tissue. Since the modulus of elasticity for a mass is a strain dependent parameter, the higher the strain level the stiffer the tissue becomes [18]. Oberai et al. [89, 90] used a nonlinear hyperelastic model of breast masses in vivo, to compute three parameters describing the tissue behavior. Their approach depended on using an inverse problem algorithm, using the displacement maps directly, to construct nonlinear tissue parameter images based on the VerondaWestman material model. They succeeded to classify nine out of ten breast tumors correctly using the described method. However, this method was computationally very extensive, and the produced imaging quality was very poor in terms of resolution. Another research group [91, 92] used a time series of strain images in vivo on non-palpable breast masses and computed viscous creep curves to estimate a strain retardation time parameter (T1). They generated creep curve for each small group of strain image pixels by plotting the estimated strain over time. Then they applied curve fitting on the data using a Kelvin-Voigt model to estimate the nonlinear parameters plotted in Fig. 2.6. They concluded that the new parameters provided a degree of discrimination between malignant and 
benign breast tumors. One limitation of their study was the usage of freehand compression, which was a potential source of error for a number of studied cases.

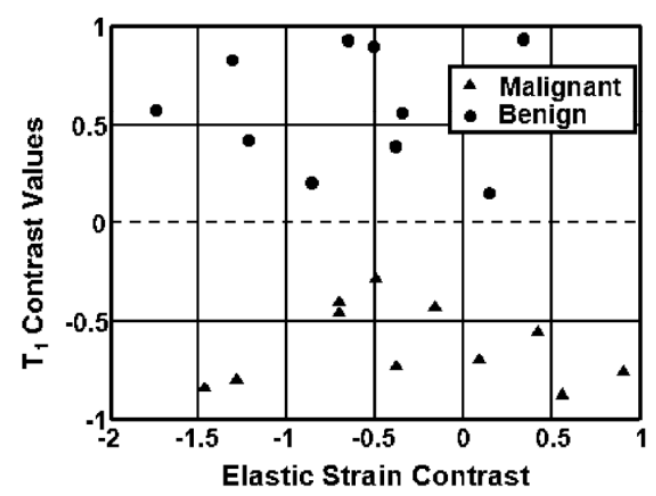

Figure 2.6 Scatter plot of patient contrast values for two nonlinear parameters providing discrimination between benign and malignant masses [91].

\subsection{Different Strain Types Imaging}

Most of the previous studies were concerned with axial strain imaging, which is one of the strain tensors that describe deformation of a material in three dimensional space. Axial shear strain is another strain tensor that has been recently reported to be feasible for elastographic imaging [49, 51, 93-95]. Figure 2.7 shows an example of axial shear imaging in vivo for a malignant case [96]. Thitaikumar et al. $[51,96]$ investigated the spatial resolution of axial shear strain elastography through simulations, and studied factors affecting the upper bounds on the resolution. They also addressed the feasibility of visualizing the characteristics of bonding at a lesion-background boundary using axial-shear strain elastography. They hypothesized that the pattern of axial-shear strain distribution around the inclusion/background interface is completely determined by the bonding at the interface after normalization for inclusion size and applied strain levels, and that it is viable to extract certain features from the axial shear strain elastograms to quantify and detect this pattern. 


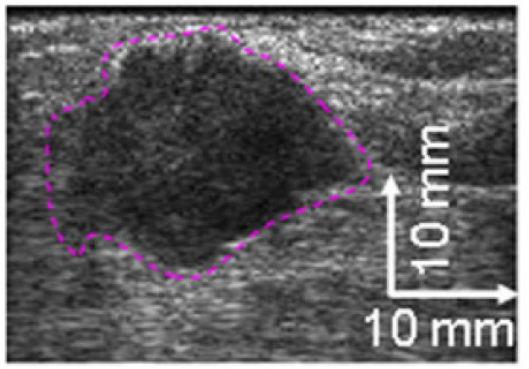

a)

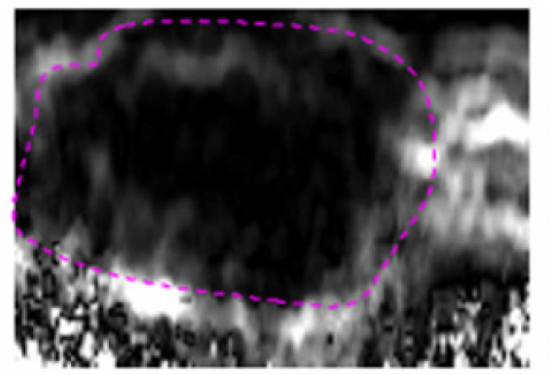

b)

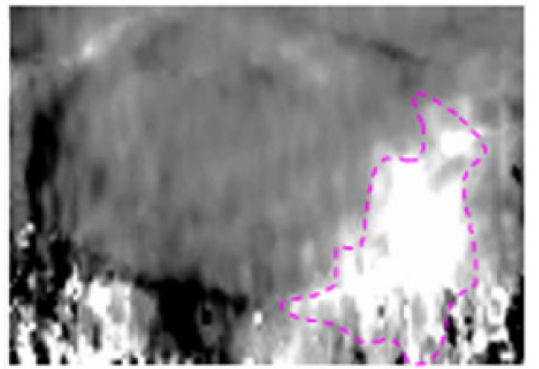

c)

Figure 2.7 a) Sonograms b) normal axial elastogram. c) axial shear elastograms of a breast cancer [96].

Thitaikumar et al. [95] investigated the potential of normalized axial shear strain area (NASSA) feature on an in vivo dataset. The authors utilized experienced observers to outline the lesions, and concluded that this strain imaging type may be added to existing routine clinical practice for better breast tumor classification. Xu et al. [49] also used the NASSA feature to classify in vivo breast cases, and suggested the axial shear strain imaging to complement axial strain and B-mode imaging in classifying breast masses. The authors limited their study to 2D imaging and suggested obtaining shear strain volumes to better predict the utility of the axial shear strain images in classifying breast tumors. Shear strain imaging has also been reported, even earlier than axial shear strain imaging $[50,97]$; yet recent studies suggested that the axial shear imaging is useful in differentiating benign from malignant breast masses, supported with in vitro and in vivo statistics. 


\section{CHAPTER 3}

\section{MATERIALS AND METHODS}

In this chapter, the materials and methods that were used in research are presented in full details. The chapter describes the signal processing steps involved, along with the elasticity imaging and volume construction algorithms. Following that, lab experiments on a breast mimicking phantom are illustrated. The experimental setup and materials used are enumerated. In addition, an in vivo study on human subjects and the used clinical elastography setup is completely described. The chapter is concluded with a new in vivo tissue classification and characterization method that is based on modeling the tissue as a nonlinear material. The main hypothesis is stated and its application on different types of breast masses is explained. Moreover, two image segmentation algorithms are described along with their application on the volumetric elastography data.

\subsection{Ultrasound Signal Processing}

The implemented elastography processing method depended mainly on a modified time domain estimator (TDE) technique $[34,35,53]$ to calculate time delays of the ultrasound signals. This technique is based on one dimensional displacement estimation analysis, i.e. along ultrasound scanning lines. Signal processing started with acquiring raw radio frequency (RF) data of the tissue under investigation using an ultrasound scanner. A research grade ultrasound unit (Sonix RP, Ultrasonix Medical Corporation, Richmond, BC, Canada) was used to acquire the RF signals at 20 $\mathrm{MHz}$ sampling frequency. Acquired data were transferred offline to a separate workstation, and processed using MATLAB (R2011b, The MathWorks inc., MA, USA).

A mechanically rocked 3D ultrasound probe (4DEC9-5, Ultrasonix Medical Corporation, Richmond, BC, Canada) was exploited for scanning. The probe consists of curved piezoelectric array elements, mounted on a micro stepper motor that rotates in a rocking motion with precise angles to accomplish scanning of the designated field of view. The array consists of 128 piezoelectric elements working at a center frequency of $6.6 \mathrm{MHz}$ and a bandwidth of $70 \%$. Figure 3.1 shows a diagram illustrating the rocking motion of the probe to generate a pie shaped volume.

The probe was initially located in good contact with the examined tissue using slight compression and also by placing a coupling ultrasound gel. The gel ensures the removal of any air 
bubbles between the probe and the tissue, and hence the removal of any stray reflections. Following that, a volumetric RF data was acquired; named pre-compression volume. Then a small amount of compression was applied for elastography to work, and another acquisition follows, this time after compression; named post-compression volume. Each RF volume consists of a number of slices, which in turn consists of a number of ultrasound scanning lines, called the A-lines. After this point the elastography algorithm is initiated to construct strain images and volumes.

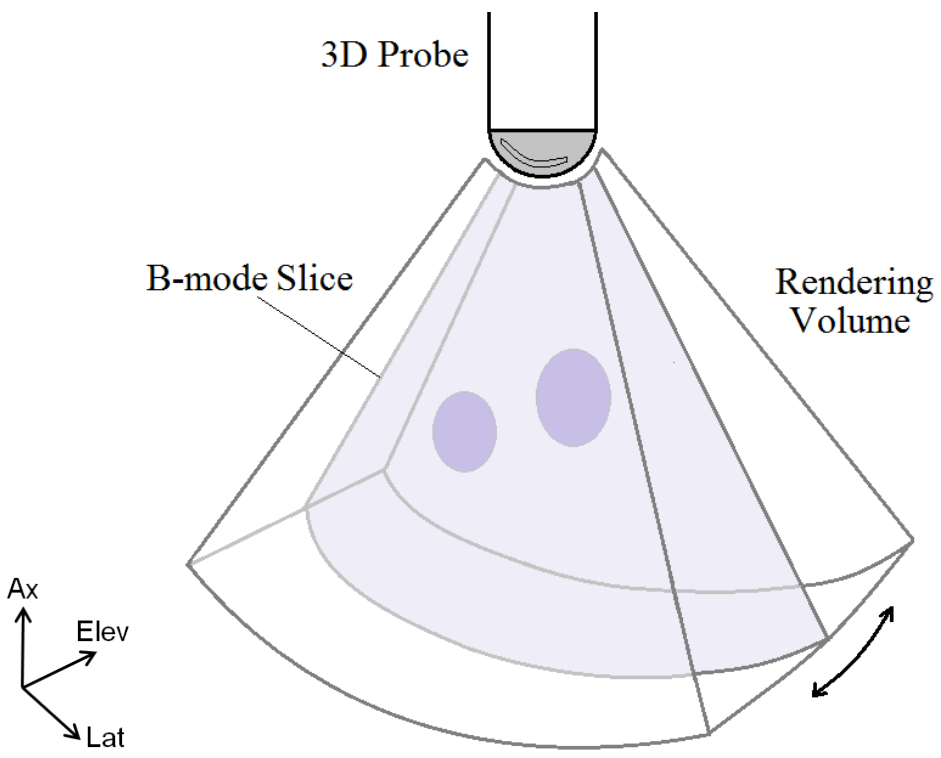

Figure 3.1 3D ultrasound probe rocking motion.

The method of acquiring the whole volume data at a fixed compression level was necessary to ensure that all the volume slices exhibited the same tissue displacement, and so were consistent with respect to the applied compression level. This facilitated the slices co-registration process, and hence elastography volume construction. Another acquisition method is to compress the tissue and at the same time acquire volume data. This method suffers from inhomogeneous distribution of compression over the volume slices, as it applies less compression on the first few scanned slices, while the later ones are more compressed, so the acquired volumes are inconsistent.

\subsubsection{Displacement Estimation}

Each acquired RF line, in both pre- and post-compression volumes, was divided into a number of temporal windows, in which a single window consisted of a number of RF samples. The used 
window size was about 40 samples or $1.6 \mathrm{~mm}$ with a window overlap of $75 \%$. A cubic spline sample interpolation with a ratio of 20:1 was applied on individual windows, to increase the displacement estimation resolution and to smooth the RF curves before the cross-correlation step. The interpolation process was found to be also reducing the noise level.
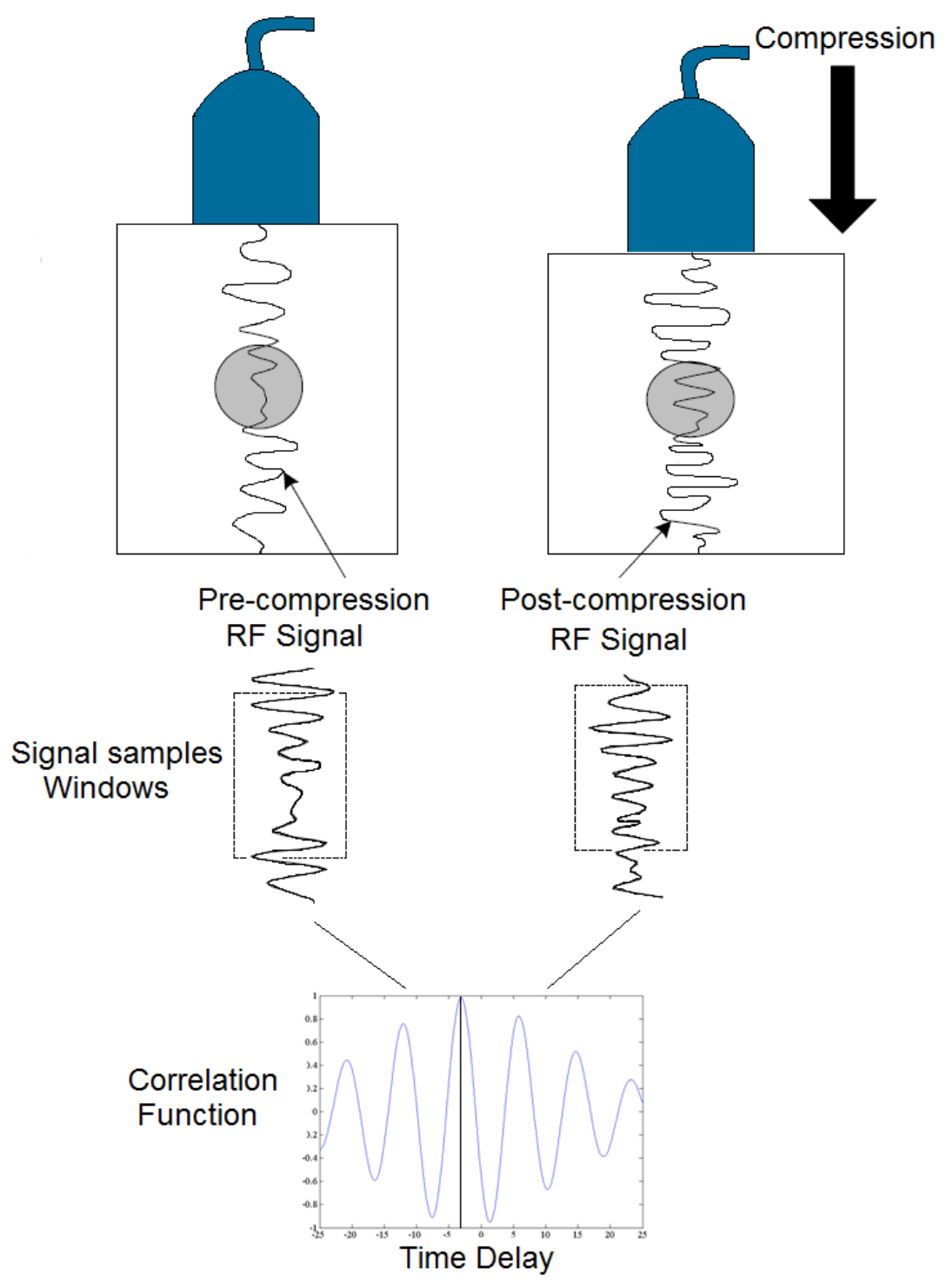

Figure 3.2 Conceptual illustration of tissue displacement estimation.

Then, normalized cross-correlation was performed between the corresponding pre-compression and post-compression RF windows, i.e. windows with the same index in the two acquired volumes. The cross-correlation function was normalized to produce accurate time delay estimates. Equation (1) was used to calculate the normalized cross correlation function [98]: 


$$
R_{X Y}=\frac{\sum_{i=1}^{W} X(i) Y(i)}{\sqrt{\sum_{i=1}^{W} X^{2}(i)} \sqrt{\sum_{i=1}^{W} Y^{2}(i)}},
$$

where $R_{X Y}$ is the correlation function, $X$ and $Y$ the pre- and post-compressions RF signals respectively, $W$ window length and $i$ an index pointing to the signal samples. The cross correlation function was calculated at a specified number of time lags between the $X$ and $Y$ signals; where the time lag equals the signal sample number multiplied by the sampling period; Fig. 3.2.

At each time lag the correlation function had a value that was stored. The location of the peak of the correlation function was then determined, which corresponded to the local time delay between pre- and post- tissue compression windows. This estimated time delay is proportional to the local tissue displacement by a factor of $c / 2$, where $c$ is the speed of sound in meters per second. The speed of sound in living tissue was assumed to be $1540 \mathrm{~m} / \mathrm{sec}$, as found in the literature [31, 34]. The calculation of local time delays was repeated for all the A-lines in the acquired data, to form a number of displacement images equal to the number of acquired volume slices.

\subsubsection{Estimation Noise}

A number of noise sources affect the displacement estimates. The main three noise sources are:

1. Tissue compression: As the tissue needs compression to produce the strain images, this compression also distorts and changes the post-compression signal itself, such that it no longer represents an exact delayed replica of the pre-compression signal. This de-correlation effect increases with elevating the applied strain level and is independent of the echo's signal to noise ratio. As a result, de-correlation false peaks often appear when a secondary peak surpasses the true peak. The de-correlation peaks appear as dots or "black and white pepper noise" in the displacement images, and can be reduced with the use of a suitable median filter $[53,99,100]$, which was used in this study. To further reduce the effect of this noise type we used a compression ratio of about $1 \%$ of the tissue's initial thickness. This small compression minimizes the dissimilarity between the pre and post-compression signals, which in turn maximizes the correlation coefficient. This moderate amount of compression was used in all experiments to compensate between contrast and noise levels $[32,48]$. 
2. Random noise brings in errors in the TDE estimation process, which are disruptive especially at small time delays. They can be reduced by applying the usual filtering processes on the raw radiofrequency data, and they depend on the ultrasound system itself. Examples of random noises are electronic and quantization noises [80].

3. Any other phenomenon, such as the lateral and elevational motions, that degrades the accuracy of the time delay estimates, and would in turn degrade the final strain estimates. Therefore, this can also introduce additional noises into the strain images [80].

To further reduce the effects of the remaining noise sources, especially the last two types, a cubic smoothing spline function was applied on the displacement images. The smoothing function had the rule of making the displacement image profile more uniform and even, as shown in Fig 3.3.

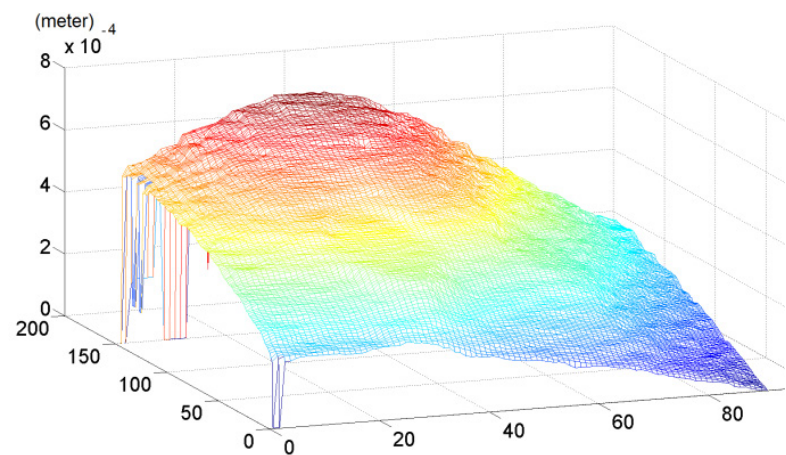

a)

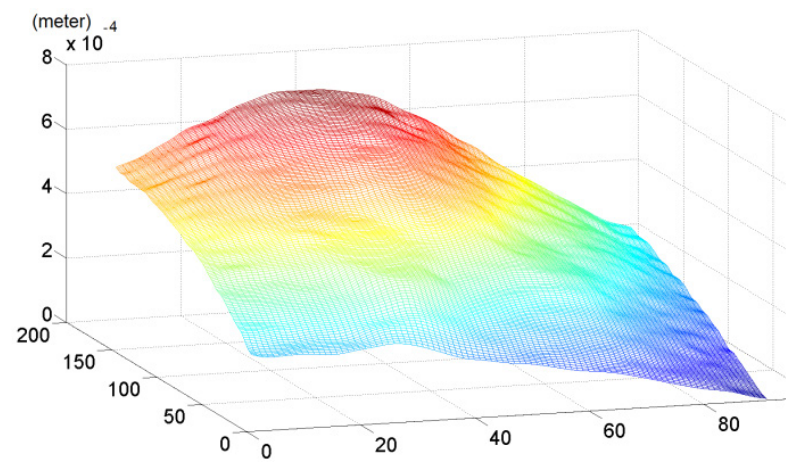

b)

Figure 3.3 Displacement image smoothing a) before smoothing b) after smoothing. Horizontal axes are in pixel units, where 10 pixels equal $2.5 \mathrm{~mm}$.

This smoothing step was very useful and essential for the gradient operator step; strain estimation step. The gradient operator is well known to be very sensitive to noise and signal fluctuations. So, smoothing facilitated a higher performance gradient operation by reducing the displacement estimates roughness, which resulted in clear strain images, as will be shown later.

\subsubsection{Displacement Values Correction}

Because of the sector profile of the 3D probe, ultrasound beam directions form an angle with the tissue axial compression direction. As a result, the displacement estimates obtained so far were 
in the beam directions not the axial compression direction, which is the correct one. The displacement image's pixel values can be corrected using that angle between the two directions, as shown in Fig. 3.4 to obtain the correct axial displacement. The correction was simply done by multiplying the previously estimated values by the cosine of the angle between the beam direction and the direction of the central ultrasound beam in the sector array probe, which we define as the "axial" direction [55]. The correction was performed on each image column individually. Please note that that angle is maximal at the far edges of the scanning area, and decreasing as we move to the center of the scanning area till it reaches zero value at the central beam. This is because the central beams become more in line with the axial compression direction.

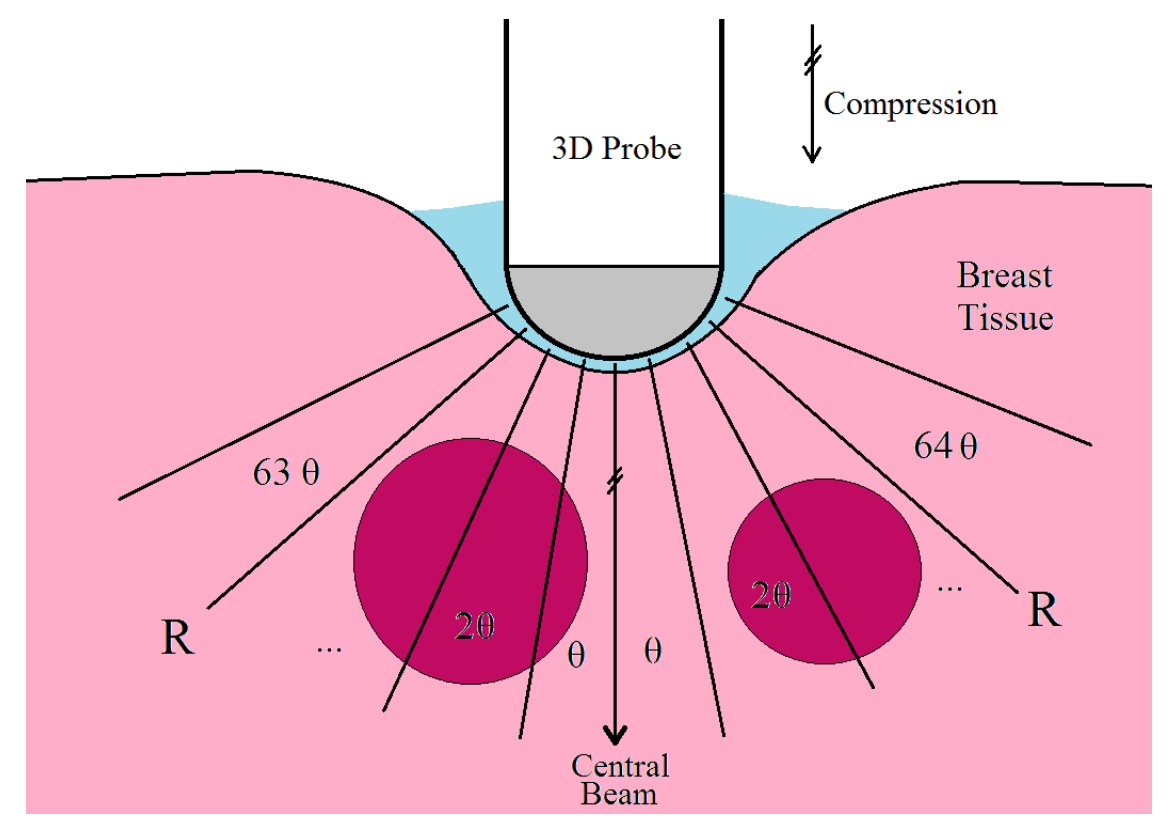

Figure 3.4 Geometry illustrating the displacement image pixel values correction.

The angel $\theta$ can be calculated from the ultrasound probe specifications, which equals the sector probe scanning angle $\left(120^{\circ}\right)$ over the number of piezoelectric elements; which equals 128 :

$$
\begin{gathered}
\theta=\frac{\text { Scanning angle }}{\text { number of elements }}=\frac{120}{128}=0.938^{\circ} \\
d_{\text {corr }}=d_{\text {est }} * \cos (n \theta)
\end{gathered}
$$


where $d_{\text {corr }}$ is the corrected displacement, $d_{e s t}$ is the estimated displacement, and $n$ is the scanning line number counting from the central beam.

The lateral displacement component $u$ can also be estimated using the same cross correlation method. But instead of comparing two windows on the same scanning line, the comparison is done on adjacent RF lines in the lateral direction. Lateral displacement estimates are very noisy compared to the axial displacement. This is a fundamental limitation with any ultrasound system due to suboptimal sampling between the RF lines, which affects elastography and B-mode imaging as well $[48,101]$. It should be noted that we have neglected the lateral components from the calculations, due to its noisy nature.

\subsection{Image and Volume Construction}

\subsubsection{Elastogram Construction}

The estimated tissue strains are derivative functions of the previously corrected displacement. To calculate the strains from local tissue displacements, the gradient operator was applied as follows:

$$
\varepsilon_{y}=\frac{\partial v}{\partial y}, \quad \gamma_{x y}=\frac{\partial v}{\partial x}
$$

where $v$ is the displacement estimate in the axial direction, $\mathcal{E}_{y}$ the normal axial strain, and $\gamma_{x y}$ the axial shear strain. The resultant strain images are known as elastograms. Strain images were usually cropped to show the region of interest. Also cropping was useful to eliminate the falsely high peaked pixel values that resulted from the application of the gradient on image edges.

The main strain component usually used in elastography imaging is the normal axial strain, as it provides direct information about tissue stiffness. Recently, axial shear strain received special research attention, as it was hypothesized that it provides some information about the bonding near the boundary between stiff and soft tissues [48, 49, 51, 95]. Both normal axial strain and axial shear strain components are independent components of the complete strain tensor that fully describes the mechanical behavior of the examined tissue.

At this stage the resultant strain images were in a rectangular shape, which is how the raw data were acquired from the ultrasound unit. As mentioned earlier the ultrasound probe was a sector 
probe, and the resultant images have to be in a sector shape too. In order to obtain an accurate strain image, a correction process had to be performed on the image pixel's locations. That process is known as scan conversion, and it consists of two sub-steps:

1. Pixel location correction: is the process of transforming the image layout from the memory saved rectangular shape to the correct sector shape. The process includes transforming the image's pixels from polar directions to Cartesian directions, using equation 4.

$$
\begin{aligned}
& A x=R * \cos (n \theta) \\
& \text { Lat }=R * \sin (n \theta),
\end{aligned}
$$

where $A x$ and Lat are the new pixel locations in the axial and lateral directions, $R$ is the probe radius plus the imaging depth, $n$ is the scanning line number counted from the center line, and $\theta$ is the angle between the processed line and the central line, as in Fig 3.4.

2. Interpolation: The number of pixels in the resulting sector image is always larger than that of the rectangular image, as a result of transformation. So inter-line pixel interpolation had to be done to fill the empty space between the lines. The average of a fixed block size of pixels was used as an interpolation mechanism. The remaining unfilled part of the resultant image was padded with zero valued pixels.

Figure 3.5 shows an example of the application of scan conversion on strain images. The image in Fig. 3.5 a) presents elongated solid inclusions inside a soft phantom. The inclusions cross section should be circular, which was the corrected shape of the inclusions after scan conversion, Fig 3.5 c). 


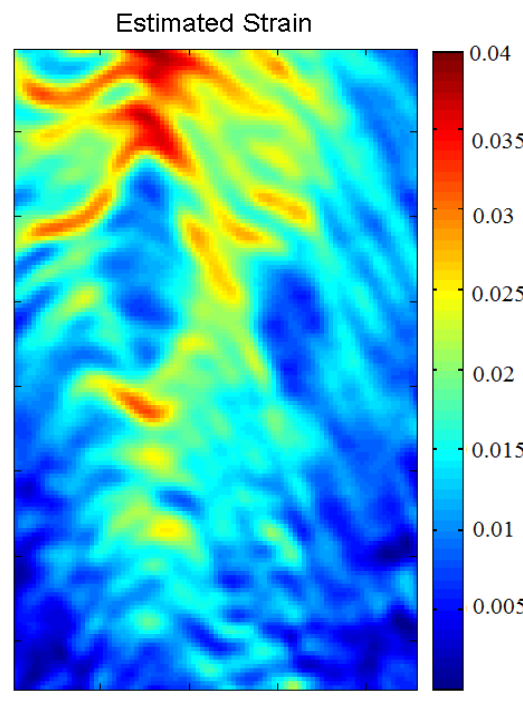

a)

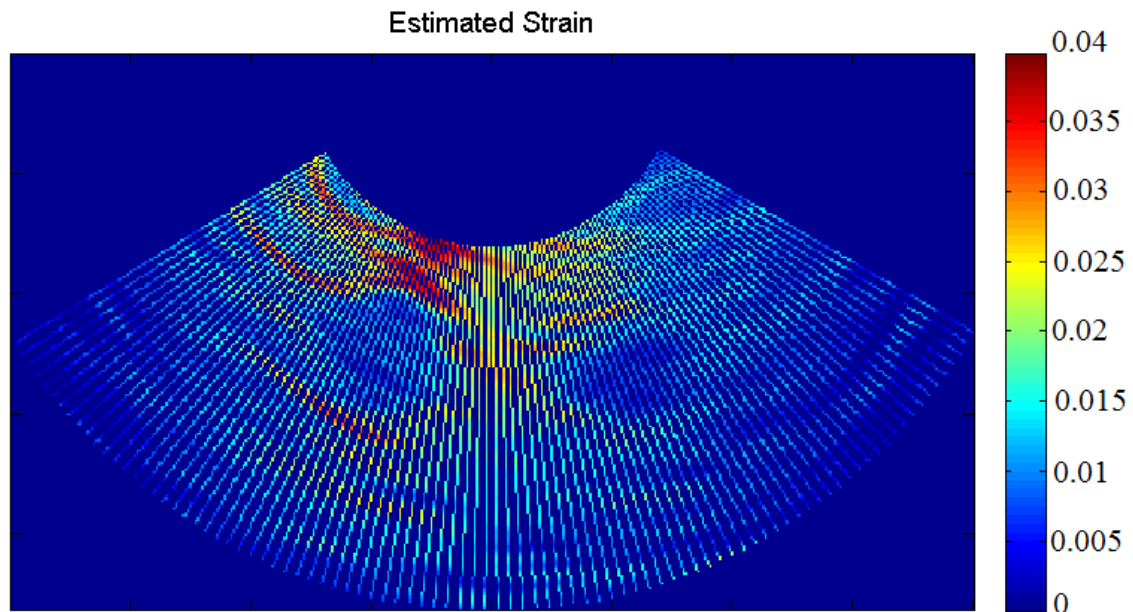

b)

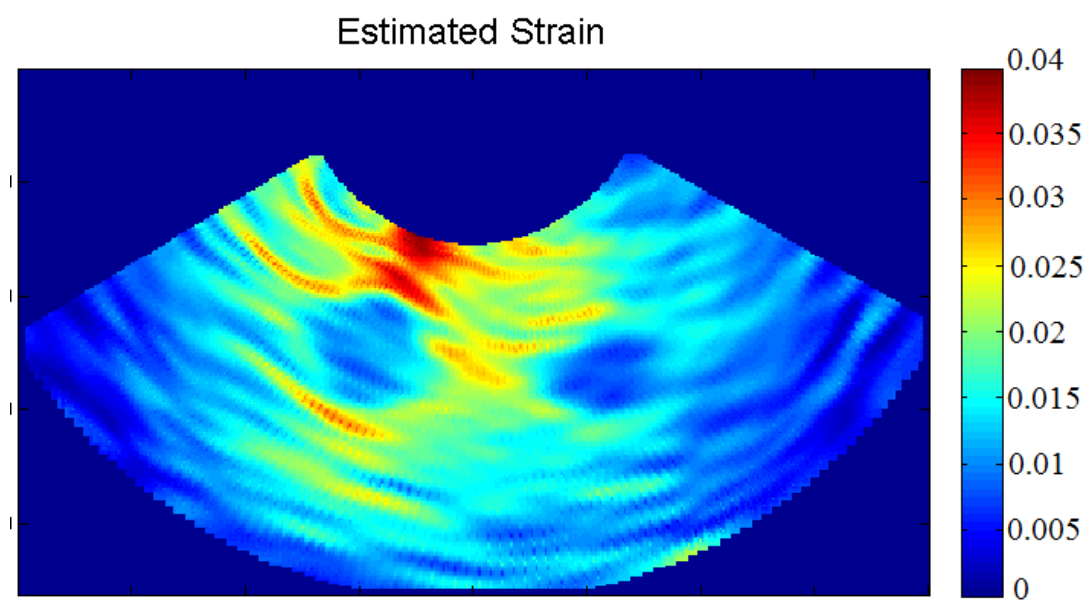

c)

Figure 3.5 Scan conversion of a strain image: a) Before correction. b) After transformation. c) After interpolation. Color bars represent tissue strain values.

\subsubsection{Derived Strains Construction}

Using the normal axial strain and axial shear strain, other useful strains can be calculated based on these two components. The new strains calculated were: First and second principal strains; maximum shear strain and Von Mises strain. The relations defining those strains are [102]: 


$$
\begin{gathered}
\varepsilon_{1}=\frac{\varepsilon_{y}}{2}+\sqrt{\left(\frac{\varepsilon_{y}}{2}\right)^{2}+\left(\frac{\gamma_{x y}}{2}\right)^{2}}, \\
\varepsilon_{2}=\frac{\varepsilon_{y}}{2}-\sqrt{\left(\frac{\varepsilon_{y}}{2}\right)^{2}+\left(\frac{\gamma_{x y}}{2}\right)^{2}}, \\
\gamma_{\max }=2 \sqrt{\left(\frac{\varepsilon_{y}}{2}\right)^{2}+\left(\frac{\gamma_{x y}}{2}\right)^{2}}, \\
V M=\sqrt{\frac{3}{2}\left(\varepsilon_{1}{ }^{2}+\varepsilon_{2}{ }^{2}\right)-\varepsilon_{1} \varepsilon_{2}},
\end{gathered}
$$

where $\mathcal{E}_{1}, \mathcal{E}_{2}$ are the first and second principal strains respectively, $\gamma_{\max }$ the maximum shear strain, and $V M$ the Von Mises strain.

We may refer to the first principal, maximum shear and Von Mises strains as derived strains in the following sections, because they were originally calculated from the normal axial strain and axial shear strains. Please note that we have neglected the lateral component of the displacement from the calculations, as mentioned earlier. The derived strains calculations were performed on pixel level, in which pixels with the same index in both normal axial strain and axial shear elastograms were substituted into Eq. 5 to produce the corresponding pixel in the resulting derived strain elastogram.

The derived strains incorporate information regarding both tissue stiffness and boundary bonding between soft and stiff tissues, with varying degree according to the strain type used. We will show that they can provide an enhanced boundary between the stiff lesion and the surrounding soft tissue, especially the maximum shear elastogram. This can be very useful in facilitating an automated segmentation process of the lesion

\subsubsection{Volume Construction}

At this stage of processing, strain images corresponding to volumetric strain slices were calculated and ready to construct strain volumes. For each strain type, strain slices were coregistered together, by ensuring that the slices contain the same region of interest. This was done by cropping strain slices with the same cropping window. Following that, two steps had to be done to construct the correct pie shaped volumes: 
1. Scan conversion: this step was very similar to the scan conversion process described in subsection 3.2.1, but this time it was applied on the axial and elevational directions. It also included pixel reallocation and interpolation between image columns. Equation 6 were used in the calculations:

$$
\begin{gathered}
A x=R_{r} * \cos \left(n \theta_{r}\right) \\
\text { Elev }=R_{r} * \sin \left(n \theta_{r}\right),
\end{gathered}
$$

where $A x$ and Elev are the new pixel locations in the axial and elevational directions, $R_{r}$ is the radius of the probe's array rotational motion plus the imaging depth, $n$ is the scanning line number counting from the center line, and $\theta_{r}$ is the angle between the processed line and the central line in the elevational direction. The geometry of the process was perpendicular to the geometry in Fig 3.4, and it was very similar to it but with different parameters.

2. Median filtering: The angle between scanning line in the axial-elevational direction were larger than that in the axial-lateral directions, i.e. $\theta_{r}>\theta$. In addition, the number of slices was smaller than the number of scanning RF lines (32 to 40 slices compared to 128 scanning lines). This made the interpolation insufficient to fill the empty space between the reallocated lines in the elevational direction, and resulted in volumes with a grid like artifact, which was not a good volume construction. A solution to this problem was to apply median filtering in the axial-elevational direction, so that to remove such empty spaces, and construct a smooth and uniform volume.

The constructed 3D volumes were displayed in MATLAB environment using a custom library based on OpenGL technology to render volumes using orthogonal plane 2D texture mapping technique. The library had alpha map and transparency adjustment capabilities, which was essential in emphasizing volume's internal structures.

\subsubsection{Algorithm Summary}

The elastography algorithm is summarized in the flowchart presented in Fig. 3.6. In summary, the process starts by acquiring pre and post-compression volumetric RF data, where the data are interpolated and then divided into temporal windows. The corresponding windows in the volumes 
are cross-correlated with each other, and the peak of the correlation function is determined, which corresponds to tissue displacement. Median filtering is applied, cubic spline smoothing and gradient operator to obtain strain images. Image cropping and scan conversion follow to obtain sector shaped images. Derived strain images are then constructed. Finally, volume slices are registered together for each strain type, scan converted and filtered to construct the pie shaped strain volumes.

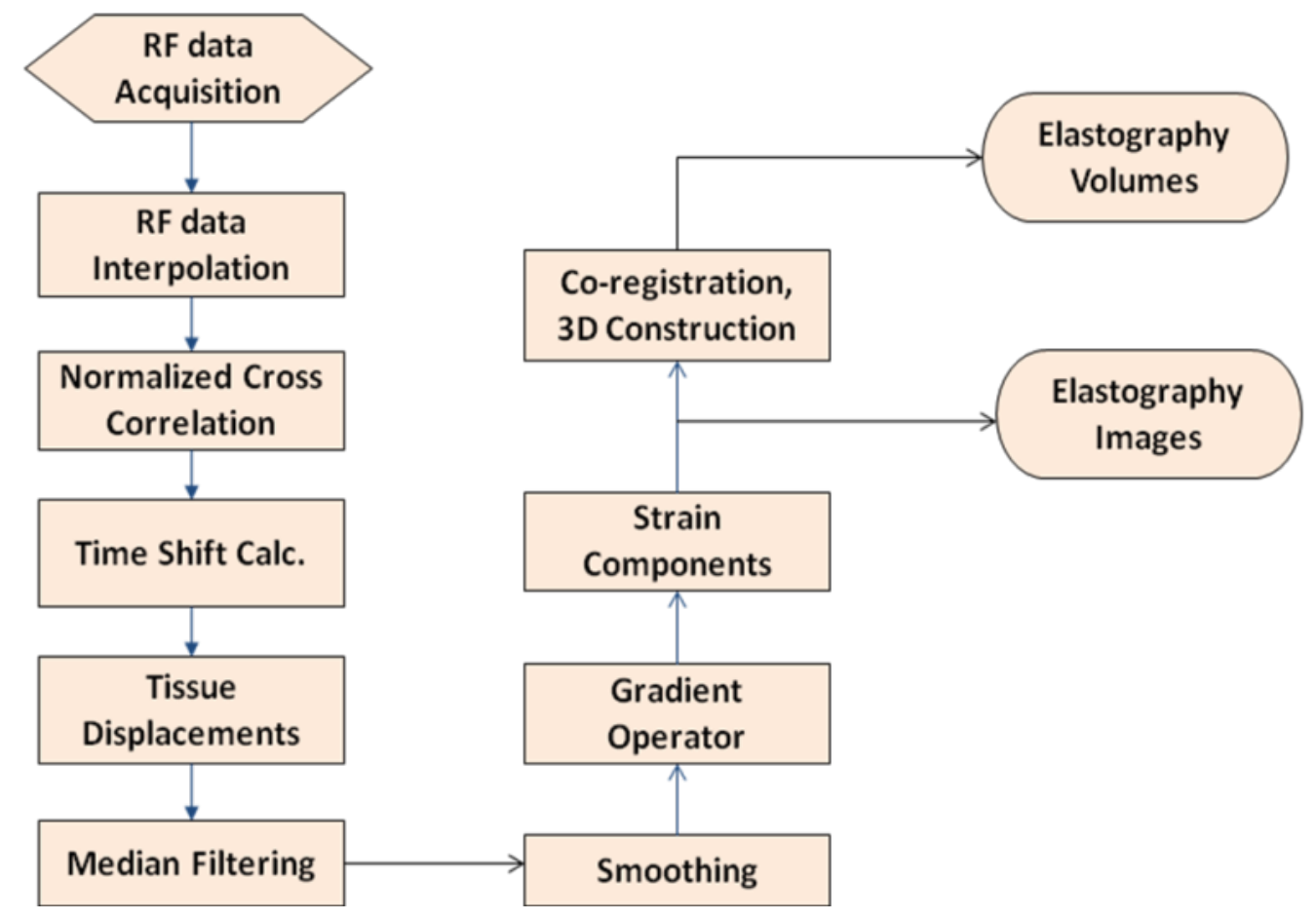

Figure 3.6 Elastography flowchart.

\subsubsection{Graphical User Interface}

A graphical user interface (GUI) program was implemented featuring most of the elastography features described earlier. The GUI was created using MATLAB GUI builder (R2011b, the MathWorks inc., MA, USA). The program facilitated rapid processing and post processing of the acquired RF data. Instead of performing each processing step individually using commands, all the steps can be performed consequently by clicking command buttons and entering the parameters through text boxes. The GUI can display both B-mode images and elasticity images in a dual display mode. The current slice, or frame displayed can be easily switched to the one next to or previous to it. The mouse pointer can be used to select a single pixel and show its brightness value, either in the B-mode or the strain image. 


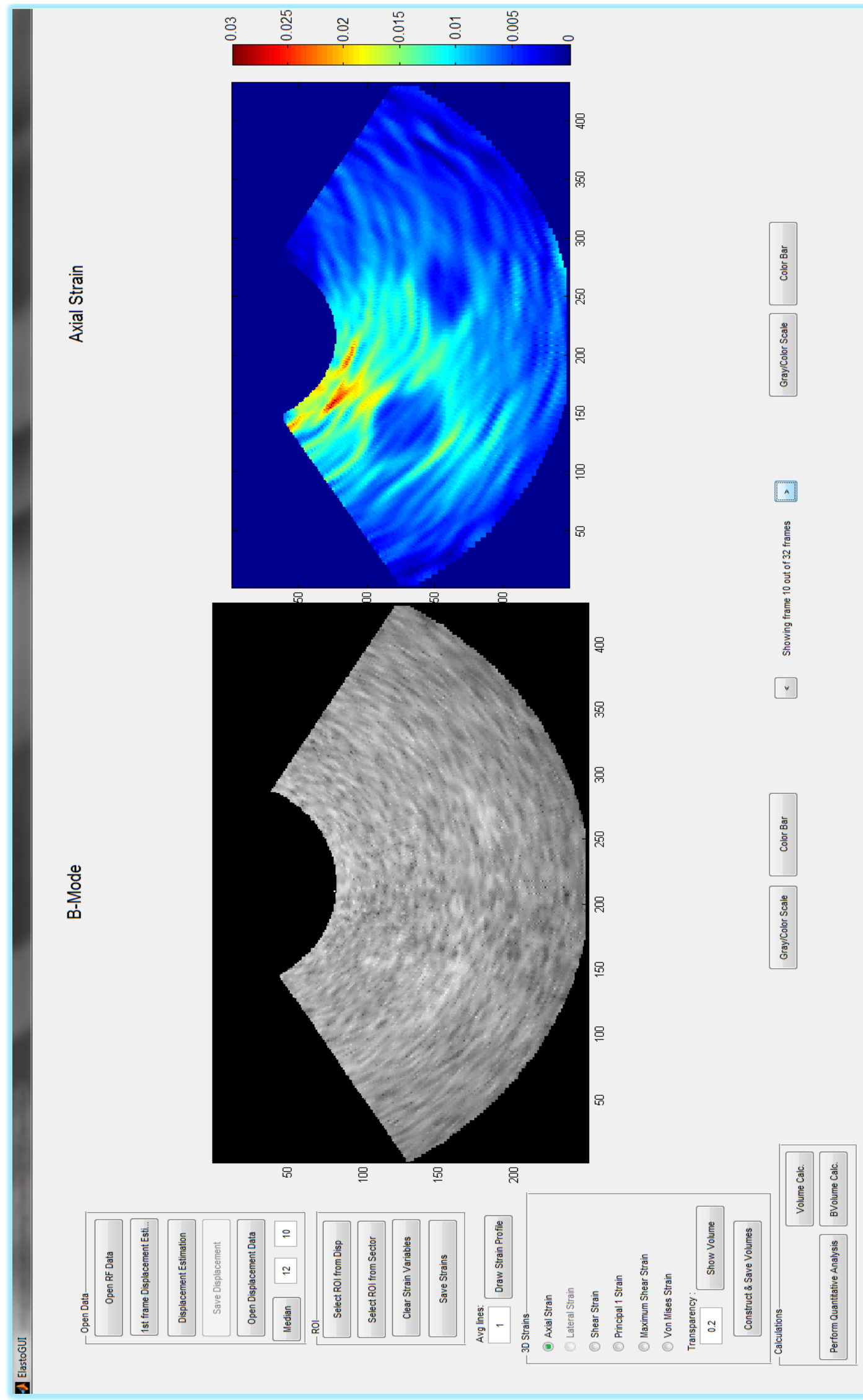

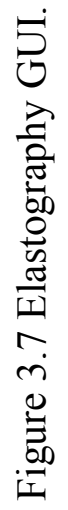


The commands are divided into four different panels, according to their role. The processing starts by opening the RF data files. A dialogue opens requesting the locations of the precompression and post-compression data files. An optional first frame displacement estimation button is provided for fast checking of the quality of the acquired RF files, otherwise another button is provided for full volume displacement estimation.

The displacement estimation process takes about 45 minutes for 40 volume slices to complete. The displacement can then be saved for future processing. Median filtering can be applied with a custom filter size through the "Median" command button. The region of interest (ROI) panel follows, in which the suspected mass is determined and the ROI is cropped from either the displacement image or the sector strain image. The choice between the two options depend on the quality of the displacement image, if the image was so clean it could be used directly for selection of the ROI, which gives a faster construction. If the displacement image was noisy, then the ROI can be selected from the sector strain image, as only limited parts of the image will be useful in this case. The strain volumetric variables can be deleted for reselection of the ROI, if needed, and a strain save option is available to save the whole constructed volumes together.

From the "3D strains" panel, the type of displayed strain can be selected through radio buttons, which switches the strain display to the selected one as soon as activated. Axial normal, shear, principal 1, maximum shear and Von Mises strain options are available for the display along with the B-mode image. The constructed final volume can be displayed in a separate window after the elevational scan conversion, by pressing the "show volume" button. The transparency parameter can be optionally entered. The volumes constructed in the final pie shape are usually huge in size and consumes the computer's memory, so they are all constructed on the fly and can only be stored at once, with the construct and save volumes command.

The calculations panel contains the quantitative analysis computation tool, in which the relative stiffness, signal to noise and contrast to noise ratios are calculated for each slice in the volume. Breast mass volume can be estimated from either B-mode imaging or strain imaging.

\subsection{D Elastography, Lab Setup}

Phantom experiments were conducted on a breast elastography phantom (CIRS, Norfolk, Virginia, USA) to test the elastography algorithm presented in Fig. 3.6. The phantom consists of an elastic material with a number of inclusions inside it; each inclusion is three times stiffer than the 
surrounding material. Elastography imaging parameters such as window length, window overlap, compression level, and interpolation ratio were evaluated and chosen for further studies based on this phantom study. The experiment setup is presented in Fig. 3.8.

a)

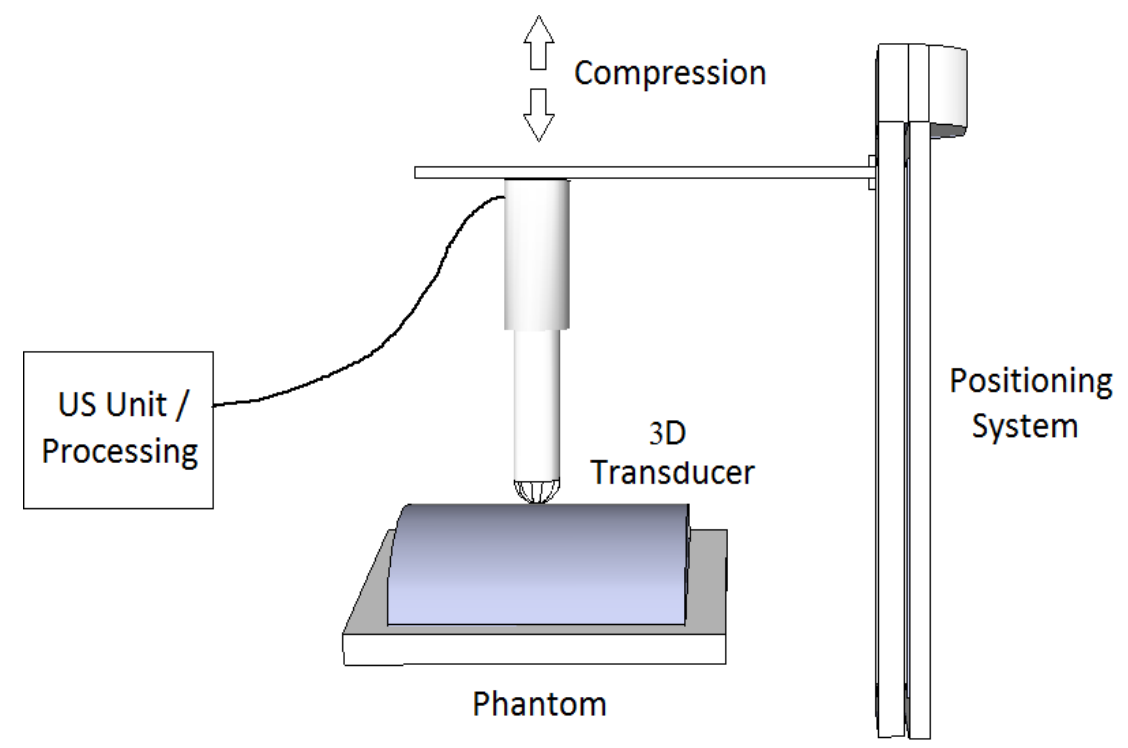

b)

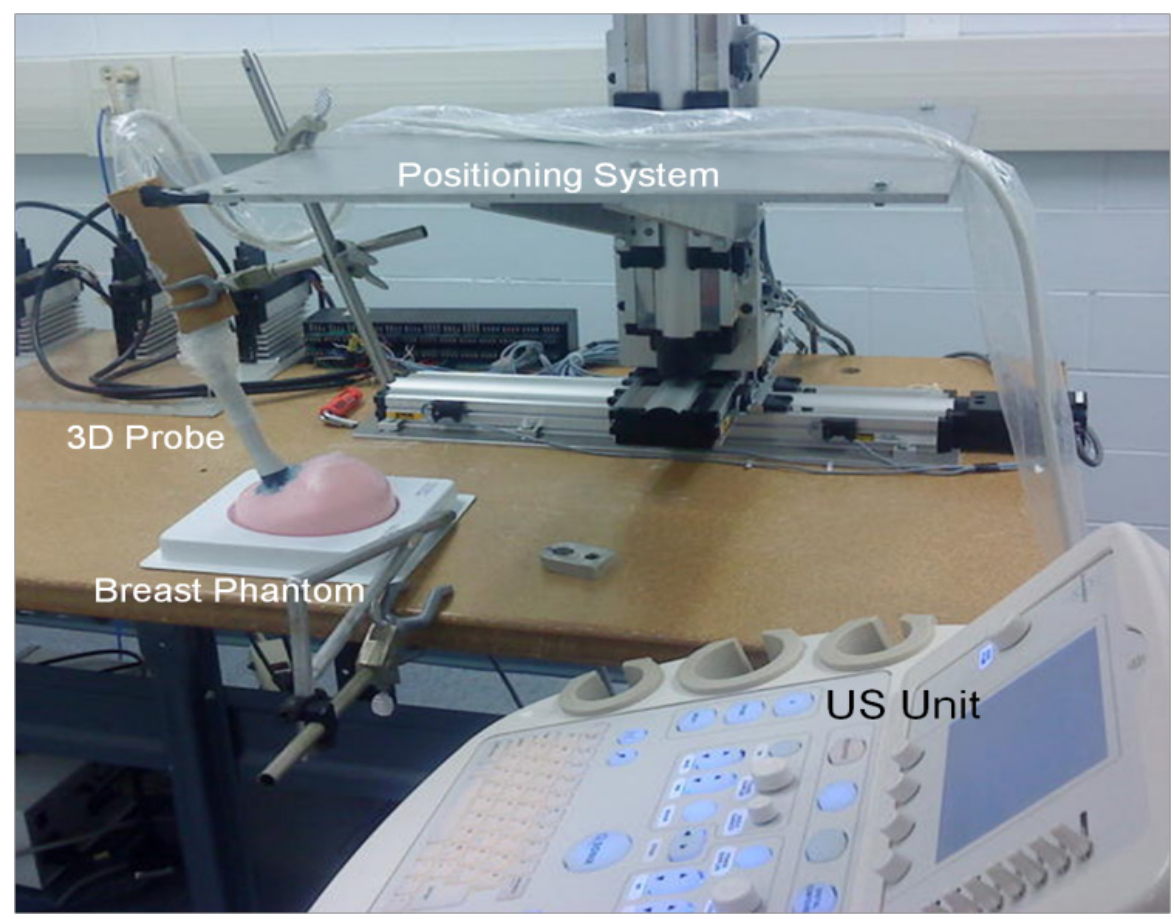

Figure 3.8 Elastography phantom experiment, Lab setup: a) schematic diagram of the components.

b) photo image. 
Compression was performed vertically using a computer controlled positioning system (Parker Hannifin corporation, OH, USA) coupled to the ultrasound probe. The positioning system facilitated compression with precise levels in steps of $250 \mu \mathrm{m}$. The 3D probe was positioned so that the stiff inclusions would be approximately at the center of the images.

Pre- and post-compression RF volumetric data were acquired for a number of compression levels and transferred to an offline workstation for processing. Strain images and volumes were constructed at each compression level, and image quality measures were performed. We found that a compression of about $500 \mu \mathrm{m}$ ( $1 \%$ of $5 \mathrm{~cm}$, the total thickness of the phantom) provides the best image signal to noise performance, which agreed well with previous studies [32, 35, 48, 101].

Regions of interest (ROIs) were first selected inside the designated tissue in the elastograms to facilitate the calculations. Two main regions were selected for evaluating image performance, the stiff inclusion and the surrounding soft tissue. To quantify the elastography imaging performance, two main standard parameters were considered, signal to noise ratio (SNR) and contrast to noise ratio (CNR) [35, 63]. Signal to noise ratio is a quantity used to describe the noise properties of the elastograms, and is defined as the ratio between mean and standard deviation of the estimated strains:

$$
S N R=\frac{m_{b}}{\sigma_{b}} .
$$

Contrast to noise ratio is used to evaluate target or lesion detectability and it combines the contrast and noise performance of the elastograms. In this study, CNR was defined as:

$$
C N R=\frac{2\left(m_{t}-m_{b}\right)^{2}}{\sigma_{t}^{2}+\sigma_{b}^{2}},
$$

where $m_{t}$ and $m_{b}$ are the strain mean values of the target (stiff inclusion) and the background (soft tissue), and $\sigma_{t}, \sigma_{b}$ are the standard deviations of the target and background strains inside the selected ROI's. Usually both parameters can be expressed in the logarithmic scale. This can be done by taking the logarithm and then multiplying by 20 .

As a measure of the accuracy of the used elastography method, relative stiffness (RS) between the stiff tissue and the background soft tissue was calculated for a number of elastograms and elastography volumes. Relative stiffness is defined as the ratio between the mean of the strain 
values for the soft $\left(m_{b}\right)$ and stiff tissues $\left(m_{t}\right)$, respectively inside the selected ROIs, and is expressed by:

$$
R S=\frac{m_{b}}{m_{t}}
$$

The calculated relative stiffness was compared with the tissue mimicking phantom's manufacturer specifications, which indicates that the inclusion was three times harder than the soft background.

Another parameter that was specifically calculated using 3D elastography is the target or inclusion volume. This was estimated using the volumetric nature of the $3 \mathrm{D}$ elastography imaging and it was calculated as the summation of inclusion's areas in each slice constituting the elastographic volume. Manual segmentation was used to obtain the area of the mass in each slice. Volume calculation was helpful in assessing the accuracy of the resultant elastograms compared with the phantom's specifications. Furthermore, volume calculations were helpful in the in vivo studies, and worked as a breast mass classification parameter as will be shown later.

\subsection{D Elastography, Clinical Setup}

Using the elastography technique described earlier, an in vivo study was conducted on ten volunteered patients at the Betty Puskar breast care center, West Virginia University. The study was approved by the institutional review board (IRB) before commencement, and an informed consent was received from each participant prior performing the ultrasound elastography examination. The patients were diagnosed having palpable or non palpable breast masses using mammography imaging modality. They were recruited for this study followed by a biopsy on the same day to confirm the study outcomes. Biopsy is an invasive method, in which a sample is aspirated form the suspected stiff mass inside the breast. This sample is then sent to the pathology lab for diagnosis, and determine if the mass was benign or malignant. Independent diagnosis of the breast mass and its type (malignant or benign) using the proposed approach was compared with biopsy results for each patient.

The mammography machine (Mammomat Novation ${ }^{\mathrm{DR}}$, Siemens medical solutions, Erlangen, Germany) located in the Betty Puskar breast care center was chosen to be utilized in this study. Its compression stage was used to perform elastography compression as well as to apply three precompression levels necessary to apply the nonlinear mass classification process. Also the stage had 
the capability of measuring the applied tissue compression force, which is essential in our study, as will be discussed later in the next section.

In order to couple the $3 \mathrm{D}$ ultrasound probe to the mammography compression stage, we designed a custom mammography compression paddle. Mammography paddles are deformable rectangular plates mainly used for the application of the compression on the breast tissue while resting on a special holder. This pressure ensures homogeneity of the tissue during imaging, and decreases the breast thickness for more efficient X-ray penetration.

The modified paddle is shown in Fig. 3.9 a). We added a movable fixture for the 3D ultrasound probe, with the flexibility of translating the probe in the $\mathrm{X}-\mathrm{Y}$ plane to acquire images and volumes of the required region of interest. Aluminum rails with movable brackets were fixed on the paddle, two rails on both sides and one perpendicular rail on the top of the paddle. A circular clamp was used to secure the probe vertically.

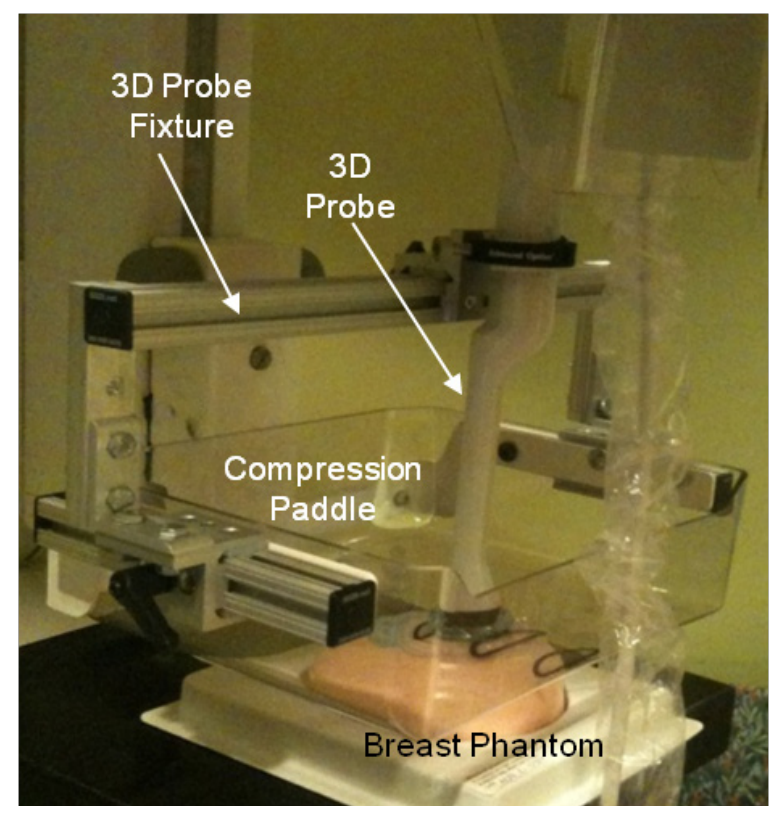

a)

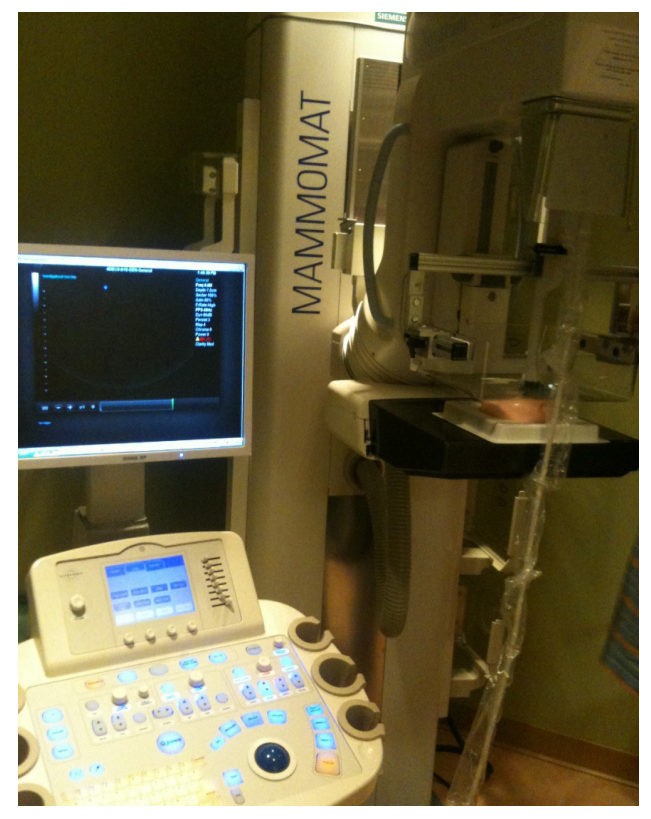

b)

Figure 3.9 Clinical study setup: a) mammography paddle with an added holder for the 3D ultrasound probe. b) Ultrasound mammography system.

Manual brakes were added to fix the probe in the required position and stabilize the probe during compression. The fixture was completely made from aluminum, which made it light weight and did not interfere with the pressure readings. The main consideration was to hold the probe so 
that it does not move, slip or rotate during the compression cycles, which ensures consistent acquisition of volumes. The fixture also allows for the ability of imaging using both ultrasound and mammography without replacing the plate, which is an additional advantage. The paddle has a slot opening, so that the ultrasound probe can have a good contact with the breast tissue.

Before commencing experiments on human subjects the modified mammography paddle was tested on the tissue mimicking breast phantom previously used in the in vitro study. This step was necessary to ensure that the paddle was able to deliver a stable and adequate amount of compression to the breast tissue. Also this test was helpful in avoiding any unexpected problems that could happen to the patients during scanning. After performing the phantom's test and ensuring that it was successful, the human clinical study was launched.

For each volunteered subject, the experiment started with locating the suspected mass under conventional ultrasound, and then the breast was positioned under the modified mammography paddle, so that the region of interest was directly under the ultrasound probe. Using the mammography compression stage, an initial level of pre-compression was applied, and volumetric $\mathrm{RF}$ data were acquired. Then an additional small compression (an amount of about $1 \%$ of the breast thickness) followed for elastography to work, and the post-compression RF data were then acquired. This cycle of the application of a pre-compression pressure (F1, F2, and F3) followed by the elastography compression $\Delta F$ is repeated three times at three different pre-compression levels to obtain three elastographic volumes at each level for the purpose of mass classification. Figure 3.10 illustrates the pre-compression cycle used in the clinical study. This repeated cycle of the application of pre-compression levels is called multi-compression elastography.

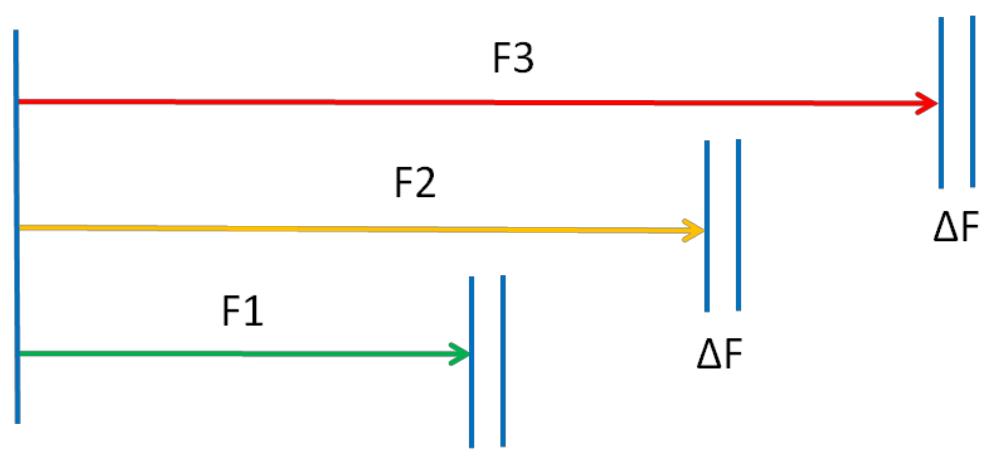

$\Delta \mathrm{F}$

Figure 3.10 Elastography multi-compression cycle. 


\subsection{Evaluating Tissue Nonlinearity}

The stress-strain curves of the breast tissues, which describe the biomechanical behavior of the tissue under different stresses, follow an exponential behavior with the malignant masses having a steeper curve than the benign one. These relationships were reported in vitro in previous studies using mechanical measurements techniques $[11,18]$. The following exponential model was used to fit the mechanical measurements [11]:

$$
\sigma=A\left(e^{m \varepsilon}-1\right)
$$

where $\sigma$ is the applied stress on the breast sample, $\varepsilon$ tissue strain, $A$ and $m$ are generalized fitting parameters. According to the authors, fitting was found to be very good, with a minimum $\mathrm{R}^{2}$ of 0.92. These studies also reported that the tissue modulus is a strain dependent parameter and the higher the strain level the stiffer the tissue becomes. Therefore, malignant masses become more rapidly stiffer than benign masses while increasing the applied stress.

Using Eq. 10, an analytical expression can be derived relating the applied stress with the strain difference between the suspected mass and the surrounding healthy tissue, as follows:

$$
\frac{\left(\sigma+A_{2}\right)^{m_{1}}}{\left(\sigma+A_{1}\right)^{m_{2}}}=\frac{A_{2}^{m_{1}}}{A_{1}^{m_{2}}} e^{m 2 m 1(\Delta \varepsilon)},
$$

or:

$$
\Delta \varepsilon=\frac{m_{1} \ln \left(\frac{\sigma}{A_{2}}+1\right)-m_{2} \ln \left(\frac{\sigma}{A_{1}}+1\right)}{m 2 m 1}
$$

where the subscript ' 1 ' refers to the suspected stiff mass, and ' 2 ' refers to the surrounding healthy tissue or fat. The last equation was plotted, representing 'stress-strain difference' curve of benign and malignant breast tissues, as shown in Fig. 3.11. It is clear that the two curves are different, with the benign curve having a higher slope than the malignant curve. This can be explained by the biomechanical behavior of the tumor, wherein the strain difference values experience relative steadiness with the increasing pre-compression force for the benign case. While for the malignant case the more compression they experience, the higher stiffness they exhibit, and hence lower mass strains were observed, hence higher strain difference values were observed with the increasing load. 


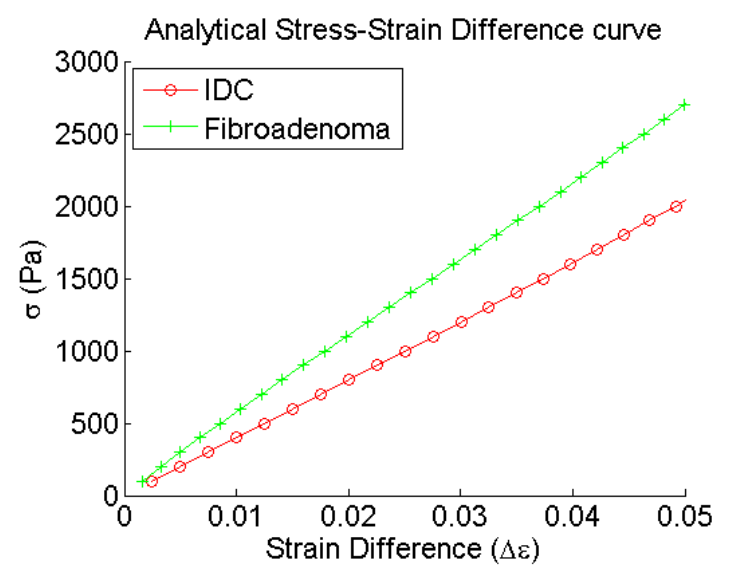

Figure 3.11 General stress-strain difference relationships for benign and malignant masses. This relationship was plotted using in vitro mechanical measurements data.

We hypothesis that the strain difference between the suspected mass and the surrounding healthy tissues becomes more significant at high compression levels. This behavior is more pronounced for malignant tissues since they exhibit strong nonlinear stress-strain behavior. We used this material nonlinearity to classify and characterize the mass type quantitatively and to differentiate between malignant and benign tissues. Figure 3.12 illustrates our hypotheses in this study. The subscripts $m$ and $b$ in the figure denotes malignant and benign, respectively. $F_{1}, F_{2}$ and $F_{3}$ denote three different pre-compression force levels.

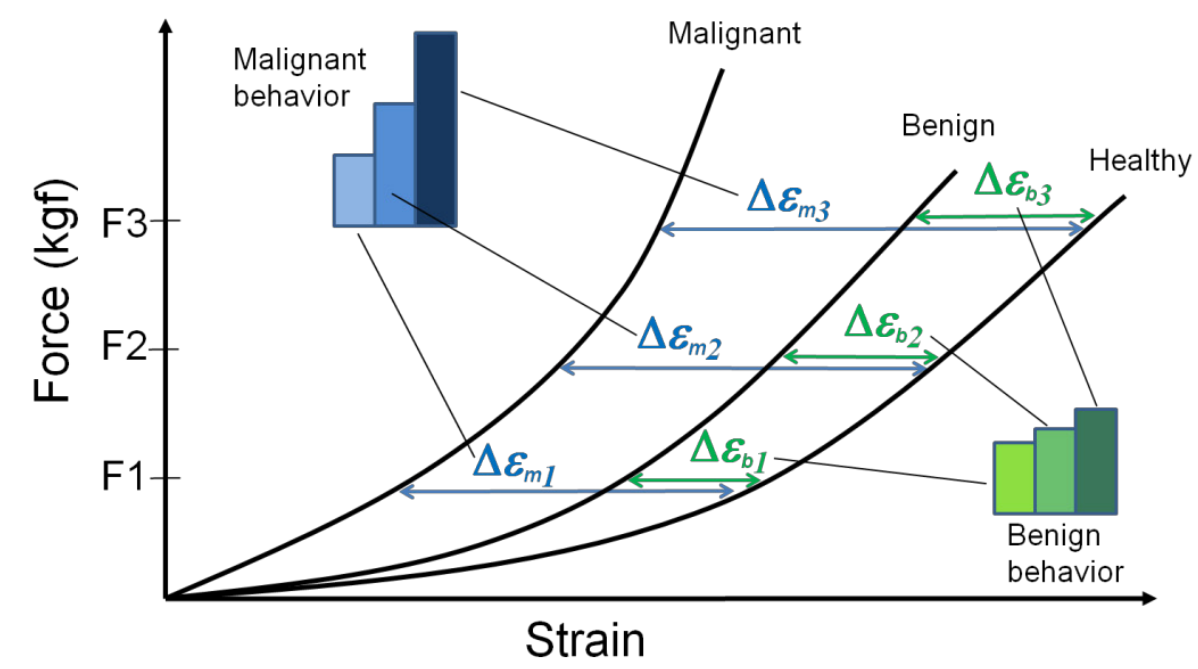

Figure 3.12 Nonlinear classification of breast masses, main hypotheses.

For each participating patient, the suspected mass was first located under conventional B-mode ultrasound, so that the required imaging field is positioned at the focus of the $3 \mathrm{D}$ ultrasound probe. 
Initial pre-compression was then applied, and pre-compression RF data was acquired. Following that, a small compression $(\Delta F)$ of about $1 \%$ of the initial breast thickness was applied, and the postcompression data was acquired at that moment, to build 3D elastography volumes. This compression cycle of pre-compression followed by small elastography compression, was repeated at three different pre-compression forces, to construct three strain volumes at each level, as shown in Fig. 3.13. A built-in force sensor in the mammography stage was utilized to measure the compression force level, in the units of kgf. The three pre-compression force levels were fixed at 2 , 3 and $4 \mathrm{kgf}$ for all cases involved in the study. These force values levels were selected to be similar to the values routinely used in regular mammography imaging. The upper limit of $4 \mathrm{Kgf}$ was not exceeded, to avoid applying excessive tissue compression.

It is worth mentioning that the sequence of applying pre-compressions started from the largest level to the lowest one, i.e. the first acquisition of the volumetric elastographic data was at 4kgf, and then acquisition at 3 and $2 \mathrm{kgf}$ followed. The reason behind this was mainly for a clinical reason, as starting the compression cycle with the highest level avoids any misplacement or rolling of the tumor under the probe during the compression cycle. Tumor rolling was observed in our initial trials when compression was applied in an increasing sequence, and this effect was eliminated by compressing the breast tissue in a decreasing sequence.

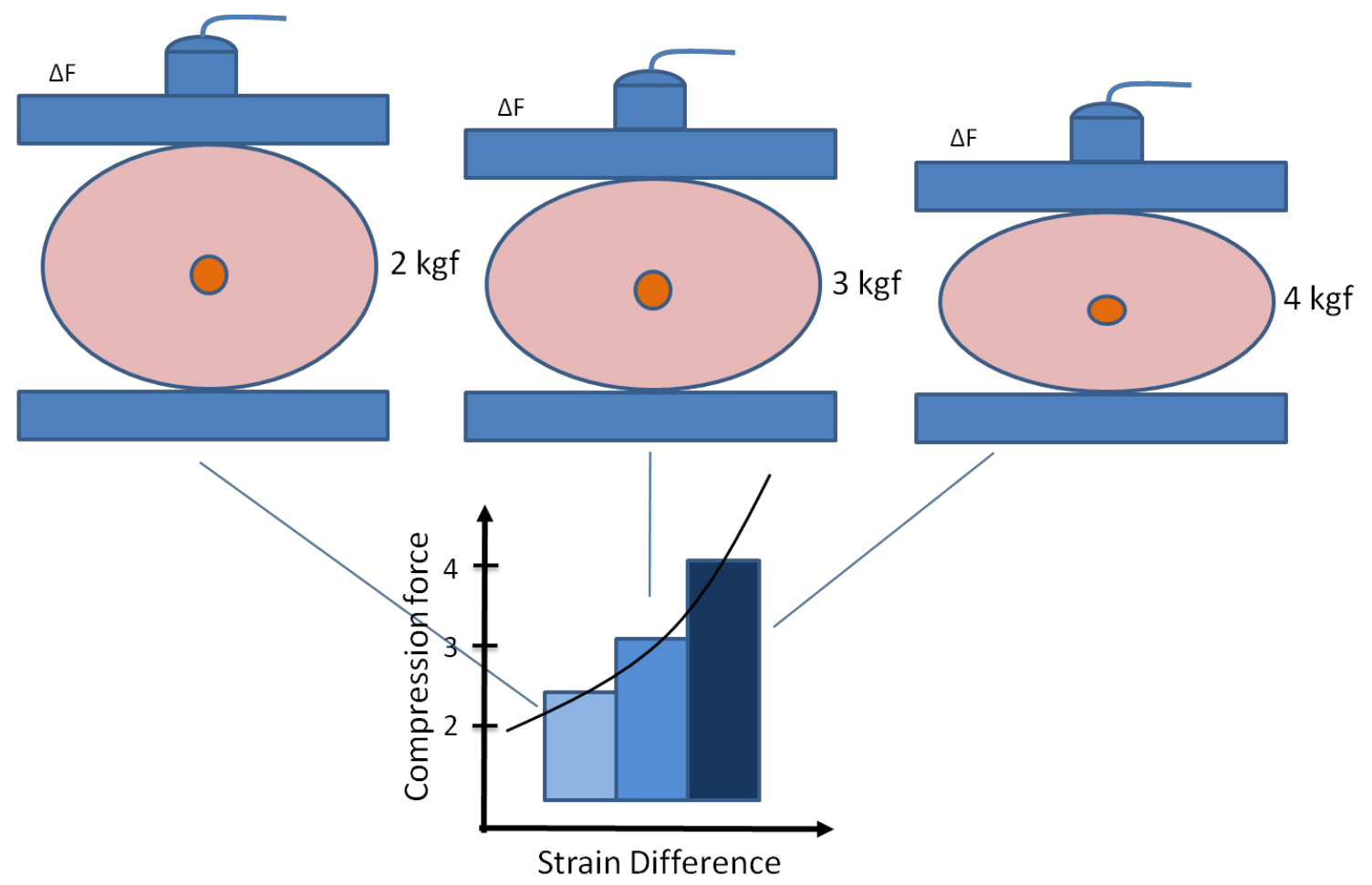

Figure 3.13 Tissue compression method. 
To achieve the in vivo mass characterization, force-strain difference curve was plotted for each case and used to characterize the breast mass. The curve was drawn at three points corresponding to the three pre-compression force $F$ levels described in Fig 3.13. We considered force values to be proportional to the true stress/pressure levels. The hypothesis states that malignant masses exhibit stronger nonlinearity than the observed for the benign masses. This behavior was also observed in the strain differences values between the masses and the surrounding healthy tissues. In order to quantify the degree of mass nonlinearity, three empirical relationships between force and strain differences were used:

1- Power-law relationship.

2- Linear relationship.

3- Logarithmic relationship.

Usually the power-law is used to describe the material behavior of some types of rubber materials and rubber blends [103]. The assumption that the shear modulus of the breast masses follows a power-law material behavior, was used for the purpose of mass characterization. The linear relationship was used for its simplicity, yet being reliable to fit the three point data. We also observed that the logarithmic relationship can appropriately fit the force-strain difference data. The three curve fittings were applied on the estimated data to describe the change degree of the strain differences with the multi-compression levels. Equation 12 describes the nonlinear elastic power law behavior [104], while equations 13 and 14 defines the linear and logarithmic fits:

$$
\begin{gathered}
F=m(\Delta \varepsilon)^{1 / n} \\
F=a(\Delta \mathcal{E})+b \\
F=c * \ln (\Delta \varepsilon)+d
\end{gathered}
$$

where $F$ is the applied force level, $\Delta \mathcal{E}$ is the strain difference between the suspected breast mass and surrounding healthy soft tissues. The parameters $m, n, a, b, c, d$ are generalized fitting parameters, which were considered to be the nonlinear parameters characterizing the breast mass type. These parameters were calculated for all cases involved in the study to estimate the threshold value that discriminates between the benign and the malignant masses. Note that the applied elastography 
compression was about $1 \%$ of the initial thickness of the breast tissue at every compression level. This was again to compromise between image quality and noise.

Summarizing the in vivo clinical study steps, a pre-compression pressure was applied on the breast tissue at three different levels. At each level an additional elastography compression was applied and the elastography algorithm was followed to estimate strains and produce elastography images and volumes at that specific level. Strain differences between stiff mass and soft tissue are calculated at each level and plotted. Curve fitting was then applied and the nonlinear curve parameter was calculated. Mass classification was then compared with the biopsy diagnosis for verification and validation.

Our new method of breast mass classification parameters was compared with the currently used elastography based parameters. One commonly used mass characterization parameter is the relative size of the mass between the elastograms and the conventional B-mode images. Usually length or area measurements are used. In this study, using 3D imaging volume calculations were feasible. As a result we used the relative volumes (RV) as an additional mass classification parameter. If the ratio between both volumes had a value bigger than one, this indicates malignancy, otherwise the mass is considered to be benign.

Another parameter that is often used to detect breast cancer is the mass relative stiffness (RS) or soft tissue/stiff mass ratio. Using 3D elastography, computing the relative stiffness was based on slice by slice calculations, in which each slice containing the mass was used to calculate a single relative stiffness value. The mean values along with standard deviations for the whole volume were plotted at each pre-compression level. Utilizing the relative stiffness ratios only as a classification parameter suffered from the overlap in values depicted by benign and malignant masses [81-83]. Multi-compression relative stiffness values can also be used to estimate the nonlinear parametric classification, in a very similar way to the usage of strain differences. Yet, strain differences provided better emphasis on the nonlinearity of stiff masses and made a larger separation margin between the malignant parameter values and the benign values, as will be shown in chapter 5 .

\subsection{Segmentation Algorithms}

After the determination of the mass's type was made, its shape and size was then emphasized using segmented 3D elastograms. This phase of our research was concerned with applying a number of segmentation algorithms on the resultant elastograms and selecting the best performing 
algorithm for further analysis. Image segmentation is the process of dividing the elastogram into a number of segments, to improve the appearance of the target breast mass and make it easier to analyze, which provides a reliable tool to determine the shape and size of the mass. In addition, the newly derived strains for elastography showed the capability of enhancing the boundary distinction between stiff and soft tissues. To prove usefulness of those new elastographic types, a direct application based on them would be the best proof. A survey on previously reported segmentation applications in elastography revealed that there are mainly two useful algorithms:

1. Gradient vector flow snake algorithm (GVF).

2. Distance regularized level-set evolution algorithm (DRLSE).

Both algorithms were tested on the breast phantom data obtained from the in vitro study and samples from the in vivo experiments. The best performing algorithm was selected for a complete in vivo imaging and analysis. In the following subsection, the basic segmentation methodology and equations are depicted, detailed description of the algorithms are out of the scope of this dissertation, and the reader is referred to the original contributions as cited in the following subsections.

\subsubsection{Snake Gradient Vector Flow Segmentation (GVF)}

A snake is an active contour defined within an image domain. The snake can move and evolve under the influence of internal and external forces. Internal forces originate from the contour itself, and are associated with the curve elasticity and bending effects. External forces come from the image's boundaries and topologies, and they are calculated based on gradient vector flow fields, where the name of the algorithm comes from. One main advantage of the GVF methods is its ability to handle broken target edges. Basically, the segmenting contour evolution is controlled by the balance of the two forces. The contour is defined as $\mathrm{x}(\mathrm{s})=[\chi(s), y(s)], s \in[0,1]$, where $\chi$ and $y$ are the image coordinates and $s$ is a parameter defining the points forming the contour. The classification of this segmentation method as a parametric method originates from the way the contour is described. The evolving contour moves around the target boundaries under the criteria of minimizing a curve energy function. 


\section{Preprocessing}

Initial preprocessing steps must be performed on the image to be segmented. Two operations are mainly prepared, pixel values normalization, and defining the image's edge map. Normalization is used to bring the dynamic range of the processed image to a known range, which was typically $[0,1]$ in our case. This unifies the dynamic range of the processed images, and avoids any dynamic range irregularities between various image acquisitions. Additionally an edge map that guides the contour evolution is defined as [72]:

$$
E_{\text {ext }}=-\left|\nabla\left(\mathrm{G}_{\sigma} * \mathrm{~S}\right)\right|^{2},
$$

where $S$ is the image (or volume slice) being segmented, $G_{\sigma}$ is a smoothing Gaussian kernel having a predefined size, and with a standard deviation of $\sigma$. The convolution in eq. 15 results in a smoothed version of the image, which reduces the effect of random noise, as well as object's boundary irregularities. As a consequence, the selection of the Gaussian kernel parameters is critical for optimal segmentation efficiency, as the improper parameter's selection may cause the contour to evolve beyond (or inside) the desired boundary. Two properties of this edge map are important to mention in the present context. First, the gradient operator emphasizes the edges and boundaries in the image, wherein this edge map exhibits larger values at the vicinity of the aimed object than at other locations, and infinitesimal values in other homogeneous regions. Second, the gradient of the edge map produces vectors pointing towards the boundaries and normal in direction to them, which further emphasizes the boundaries and guides the evolution of the segmenting contour.

\section{Contour evolution}

Operator defined initial contours, are first defined inside the region corresponding to the breast mass. A point is manually selected at the approximate center of the mass, then a circle with a radius of 6 pixels is created by the program around the selected pointed, and used as the initial contour. This initial contour is defined mathematically as $\mathrm{x}_{0}(\mathrm{~s})$ or $\mathrm{x}(\mathrm{s}, \mathrm{t}=0)$ with a higher dimensional parametric contour representation. The symbol $t$ refers to time of contour evolution, $\Delta t$ is the time step used in the numerical implementation of the algorithm.

The general contour evolution partial differential equation is defined as [70]: 


$$
\xi x^{\prime \prime}(s)-\beta x^{\prime \prime \prime \prime}(s)-v=0,
$$

where $\xi$ and $\beta$ are constants defining the contour's tension and rigidity, respectively. Both

constants are treated as two parameters controlling the segmentation process. $x$ " $(s)$ and $x$ "'” $(s)$ are the second and fourth derivatives of $x(s)$ with respect to the parameter $s$. The static external force field $v$ is numerically computed using the edge map defined by eq. 15. For further details on this evolution function, the reader is referred to the original article by Xu et al. [70].

The main model parameters are $\xi$ and $\beta$, along with the time step $\Delta t$ originating from the model's implementation. The time step $\Delta t$, controls only the speed of the contour's evolution, and does not affect the convergence of the contour evolution. The parameters $\xi$ and $\beta$ should be adjusted according to the application in hand. Nonzero value of the parameters would provide internal forces that drive the evolution of the contour to the desired location. The number of solution iteration is another important parameter to take into consideration, as it controls when the evolution stops. If the situation desires the contour to stop at an early stage (in case of weak boundaries for instance), a small number of iterations should be used, otherwise this parameter does not affect the accuracy of segmentation.

Equation 16 is iteratively solved for $x(s)$ given $x_{o}(s)$ which is the initial contour defined by the operator. The final solution of this equation results an evolved contour, delineating the object of interest; the breast mass.

\subsubsection{Level Set Evolution Segmentation}

One main advantage of active contour level set models over parametric models is their ability to represent complex contours' topologies. Topological changes, such as contour merging or splitting, can be handled in an efficient way. Level set methods can perform numerical computations on a Cartesian coordinates, without parameterzing the points on the evolved contour. The basic idea of the level set method is to represent a contour as the zero level set of a higher dimensional function, called a level set function (LSF), and formulate the motion of the contour as the evolution of the level set function. In this study, the used level set segmentation method has the additional advantage of maintaining regularity of the level set function, which ensures stable contour evolution and precise computations $[72,76,105]$. 


\section{Preprocessing}

Prior to the application of the contour model, initial preprocessing steps must be performed, using the image to be segmented. Basically, two operations are prepared, pixel values normalization, and defining an edge indicator function.

Normalization is used to bring the dynamic range of the processed image to a known range, which was typically $[0,255]$ in our case. Again, this unifies the dynamic range of the processed images, and avoids any dynamic range irregularities between various image acquisitions, which ultimately improve the performance of segmentation process.

Additionally an edge indicator function; $g$, is defined as [76]:

$$
g=\frac{1}{1+\left|\nabla \mathrm{G}_{\sigma} * \mathrm{~S}\right|^{2}},
$$

where $S$ is the volume slice being segmented, $G_{\sigma}$ is a smoothing Gaussian kernel having a predefined size, and with a standard deviation of $\sigma$. Equation 17 is very similar to eq. 15 , in terms of their common function to emphasize the image edges and incorporating the gradient function properties. The addition of 1 to the denominator of eq. 17 was to avoid singularities. The convolution in the denominator results in a smoothed version of the image, which reduces the effect of noise and object's boundary roughness. Once more, the selection of the Gaussian kernel parameters affects the overall segmentation efficiency. The gradient operator emphasizes the edges and boundaries in the image. The function $g$ exhibits smaller values at the boundaries of the aimed object and larger values at homogeneous regions of the image. This edge indicator function is directly fed to the active contour model in eq. 17, described in the next subsection.

\section{Level set curve evolution}

Operator defined seeds, or initial contours, are first selected inside the region corresponding to the breast mass. This process is very similar to the initial contour selection using the GVF algorithm. A point is manually selected at the approximate center of the mass, then a $6 \mathrm{X} 6 \mathrm{kernel}$ is created by the program around the selected pointed. This seed, denoted as $\phi_{0}(\mathrm{x})$ is set as the zero level with a higher dimensional level set function. The seed is defined as $\phi_{\mathrm{o}}(\mathrm{x})= \pm \mathrm{c}$, where $c$ is a positive constant, $x$ is a point belonging to a region $R_{o}$ in the domain of the image. The \pm sign 
reflects the relative location of the point $x$ with respect to the evolving contour. The common assumption is that the level set function has negative values inside the evolving contour, and positive values outside.

The general contour evolution partial differential equation, which is also known as the level set evolution equation is [71] :

$$
\frac{\partial \phi}{\partial t}=f|\nabla \phi|,
$$

where $\phi$ is the level set function, $f$ is the speed function that is defined to guide the motion of the contour. Equation 18 is iteratively solved for $\phi(x)$, given $\phi_{0}(x)= \pm c$, which results in the evolved contour delineating the object of interest.

In this study, we used a variant of the level set evolution methods; named distance regularized level set evolution (DRLSE). One of its applications is an edge based active geometric contour model, which is defined as [76] :

$$
\frac{\partial \phi}{\partial t}=\mu \operatorname{div}\left(d_{p}(|\nabla \phi|) \nabla \phi\right)+\lambda \delta(\phi) \operatorname{div}\left(g \frac{\nabla \phi}{|\nabla \phi|}\right)+\alpha g \delta(\phi)
$$

where $d_{p}$ is a function describing the potential for distance regularization, $\delta_{\varepsilon}$ is the Dirac delta function and $g$ is the edge indicator function. The right hand side of Eq. 19 contains three distinctive energy terms. The first term is related to the distance regularization energy that maintains the stability of the level set evolution. The second term is associated with a contour's line energy that controls the evolution near the object boundaries. The third term is associated with a contour's area energy that speeds up the evolution process, especially when the initial contour is distant from the mass boundaries. For further details on this level set evolution function, the reader is referred to the original article by Li et al [76].

The main model parameters are $\mu, \lambda$, and $\alpha$, along with the time step $\Delta t$ originating from the implementation of the model. Parameters $\lambda$ and $\mu$ can be fixed in our current application, as the model is insensitive to the choice of their values. The time step $\Delta t$, controls only the speed of the contour's evolution, and does not affect the evolution convergence. Number of solution iteration is also an important parameter that controls when to stop the contour's evolution. The parameter $\alpha$ 
should be adjusted according to the application in hand. A nonzero value of $\alpha$ would provide external force, that drives the evolution of the contour to the desired location.

Two main problems can occur if the parameter $\alpha$ was inadequately selected. First, for objects with faint boundaries; as in the case with some elastography images, the value of $\alpha$ can cause some boundary leakage, in which the evolving contour may go beyond the desired object's boundary in some regions. This effect can be avoided by setting a small value of $\alpha$, which was chosen to be -3 throughout this study. Second, the final evolving contour may slightly diverge from the desired boundary, because of the weighted area term's shrinking or expanding effect (third term in Eq. 19). Such small deviation can be reduced by performing a post-processing step. This step makes the contour converge more accurately to the desired location, by refining the level set function with a further evolution for a few iterations, but this time the value of $\alpha$ was set to zero. At this stage, the final outlining mass's contour is obtained.

\subsubsection{D volume construction and volume calculation}

Using either one of the segmentation methods, the final contour of the segmented object is then obtained and stored. The area enclosed by the final contour is filled with pixel values of one, while the remaining areas are filled with zeros, which constitute a binary mask. This mask is projected on the original elastography image (slice). The projection results in a segmented slice showing only the delineated object, which corresponds to the stiff breast mass.

Following that, the resultant segmented elastography slices are used to construct the segmented volume. Scan conversion operations in the elevational direction are performed to produce a pie shaped volume, representing the real scanned volume. Inter-slice smoothing is performed by convolving each image in the axial-elevational plane with a Gaussian low pass filter. The last smoothing operation made the constructed volume less distorted and better visualized by decreasing the inter slice's sharp transitions.

After the final segmented volume is created, the outline of the mass in each slice of the volume is found. Each slice's outline was checked for being empty or not, as empty outlines represent slices that does not show the mass, i.e. the mass did not appear in the corresponding slice. The area of each produced 'non empty' outlines is then calculated, and used to compute the mass's volume by adding all slices' areas together. This calculated volume is in cubic pixels units, which can be easily converted into cubic millimeter units. 


\section{CHAPTER 4}

\section{TISSUE MIMICKING PHANTOM STUDY}

In this chapter, the in vitro breast phantom study is presented. The $2 \mathrm{D}$ and $3 \mathrm{D}$ elastography imaging method was applied on the breast phantom, and imaging results are demonstrated. The primary aims of this study were to test the proposed 3D imaging method and to implement a solid framework for the following in vivo study. Furthermore, image quality parameters were used to determine the optimum acquisition factors, such as compression ratio, window width, overlap...etc. Additionally, the derived strains were estimated, evaluated and used to construct new types of elastography that can be used to enhance the boundaries between stiff and soft tissues, both in vitro and in vivo studies.

The method described in subsections 3.1 and 3.2 has been applied on the breast phantom using two methods of compression; freehand and mechanical. Freehand compression was applied with a linear ultrasound transducer that is capable of acquiring 2D frames only. The purpose of such experiment was to test whether elastography works with the phantom or not, and also to tune the initial signal processing parameters. On the other hand, mechanical compression was accomplished via a motorized positioning system with a 3D sector ultrasound probe. This experiment was performed to construct 3D strain imaging volumes and tune the algorithm for such applications. 3D strain imaging was not accessible through freehand compression, as the transducer sector array movement is taking place while acquiring the RF slices and compressing the tissue at the same time. This would result in non-uniform compression over the acquired volume, in which less compression is applied on the first few scanned volume slices, while the later slices would exhibit more compression, so the acquired volumes are inconsistent in this case. To unravel this problem, a motorized positioning system was used to move the ultrasound 3D probe while acquiring volumes. Two volumes were acquired; pre- and post- tissue compression to construct one consistent 3D elastography volumes. This scheme of acquisition has the additional advantage of precisely determining the amount of compression applied on the tissue, which was used to investigate the algorithm quantitatively. Throughout this research, imaging was performed using a research grade ultrasound unit (Sonix RP, Ultrasonix Medical Corporation, Richmond, BC, Canada). 


\subsection{Application of Elastography Technique}

\subsubsection{Breast Phantom}

The used breast phantom is a commercially available phantom (CIRS, Norfolk, Virginia, USA), that is made of soft material mimicking in both shape and size, the average human breast laying in the supine position. Phantom contains a number of spherical masses (inclusions) that are approximately three times stiffer than the background soft tissue. The inclusions are distributed randomly through the breast tissue, and their sizes range from 2 to $10 \mathrm{~mm}$ in diameter, Fig 4.1 [106].

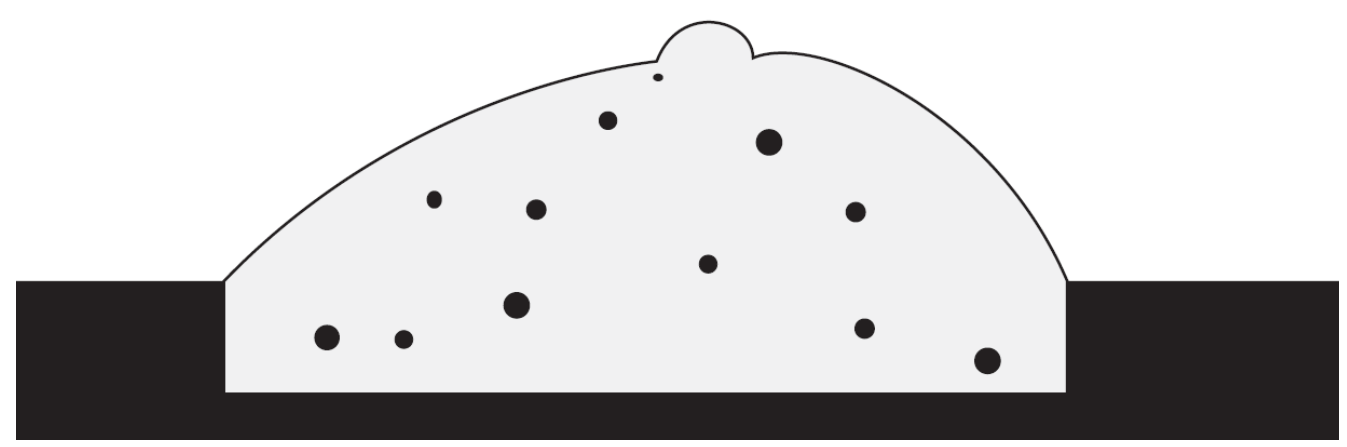

Figure 4.1 Breast phantom construction [106].

\subsubsection{D Elastography Imaging}

The phantom was imaged with a linear array transducer to produce conventional B-mode ultrasound imaging. RF data were then acquired before and after compression, at the same scanning position and the associated elastograms were constructed, as shown in Figure 4.2. Two stiff inclusions can be merely seen in the B-mode image; Figure 4.2 a). While in the normal axial elastogram the inclusions can be noticed very easily as two black circles, Figure 4.2 b). Using Eq. 3.3 in chapter 3, the axial shear strain was calculated and shown in Fig 4.2 c). Axial shear elastogram has a remarkable feature, in which two bright edges appear around the inclusion at a certain angle, and two dark edges are on the opposite sides of the inclusion. This angle that the edges appear at, is proportional to the shear strain exhibited by the inclusion due to axial compression. A virtual light source is illuminating from the lower right corner of the axial shear image, and the light is reflected over the cliffs formed by the inclusions' strain changes.

The normal axial strain and axial shear strain elastograms are the common types for elastography imaging found in the literature. The new derived strains were calculated using Eq. 3.5. 
The new strain types to be used in elastography are: first and second principal strains, Von Mises and maximum shear strains, Fig. 4.2 d-f). The figure shows a noticeable degree of inclusion boundary enhancement, and the stiff tissue can be discriminated from the soft tissue more easily. This explanation behind this effect will be discussed later in this chapter. The second principal elastogram was very similar to the axial shear elastogram and therefore was not demonstrated in these images.

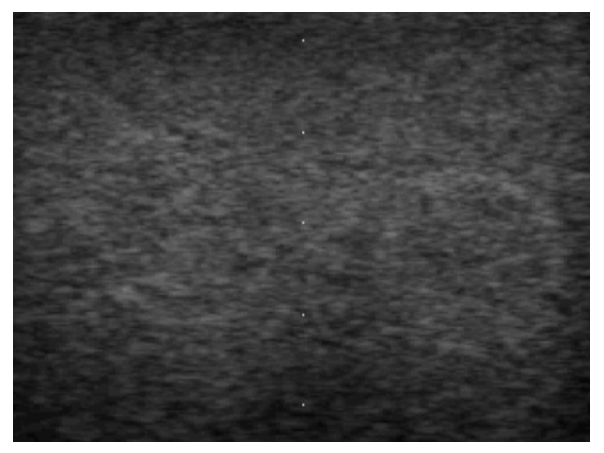

a) B-mode image

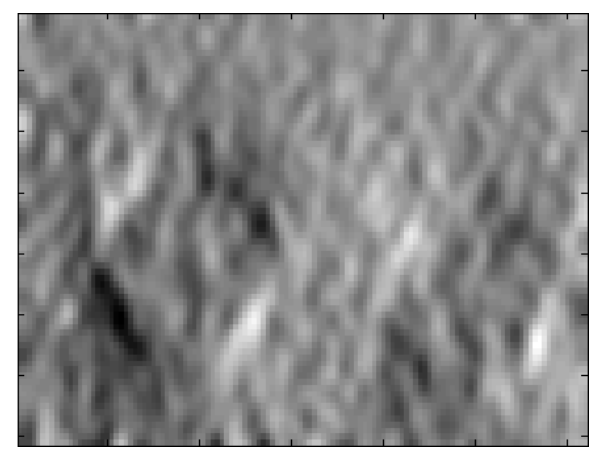

c) Axial shear elastogram

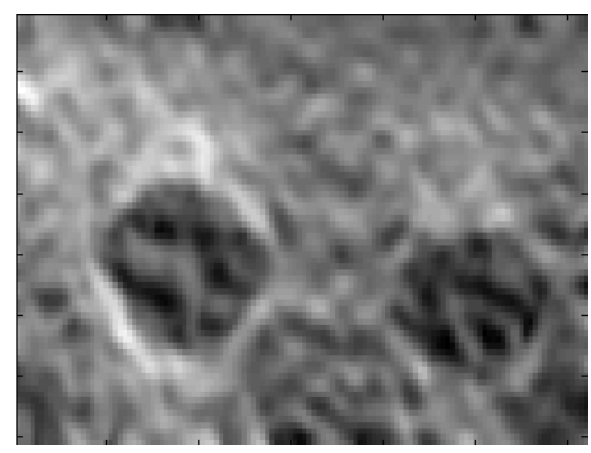

e) Von Mises elastogram

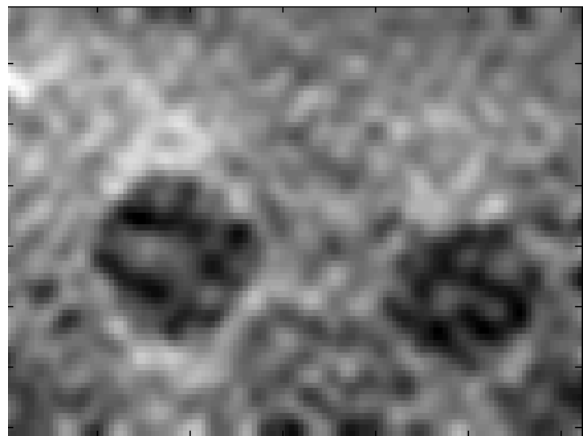

b) Axial stain elastogram

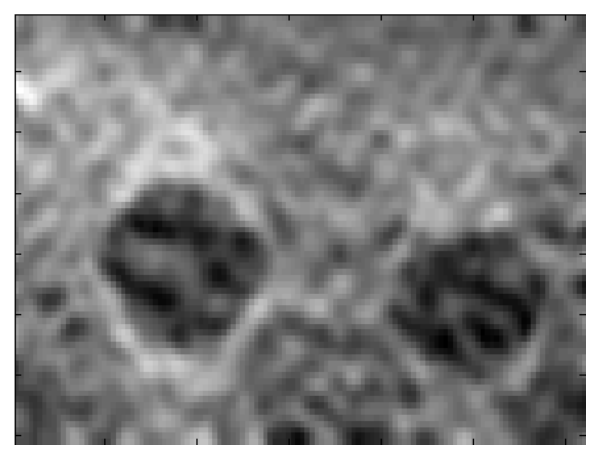

d) First principal strain elastogram

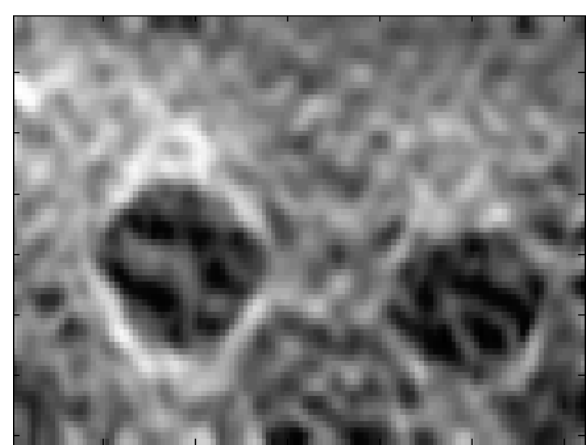

f) Maximum shear elastogram

Figure 4.2 2D Breast phantom imaging. 


\subsection{D Imaging}

\subsubsection{D elastography}

The method described in section 3.2 has been applied on the breast phantom using a motorized compression with a positioning system to apply compression. The 3D sector ultrasound transducer was used in this study to acquire volumetric RF data. The displacement estimation and strain calculation was performed on a slice by slice basis through the volume. The acquired volumes consisted of a total of 32 slices. The resultant B-mode slice and volume along with the associated normal axial strain elastography are shown in Figure 4.3, where the imaging direction is from the top of the shown images and volumes, i.e. along the shown axial (Ax) direction. Please note that throughout the dissertation, the axial, lateral and elevational imaging directions will be denoted by Ax, Lat and Elev perpendicular axes.

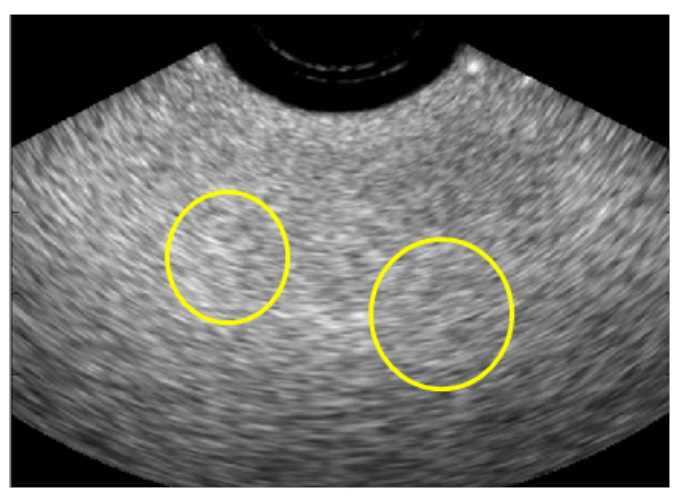

a)

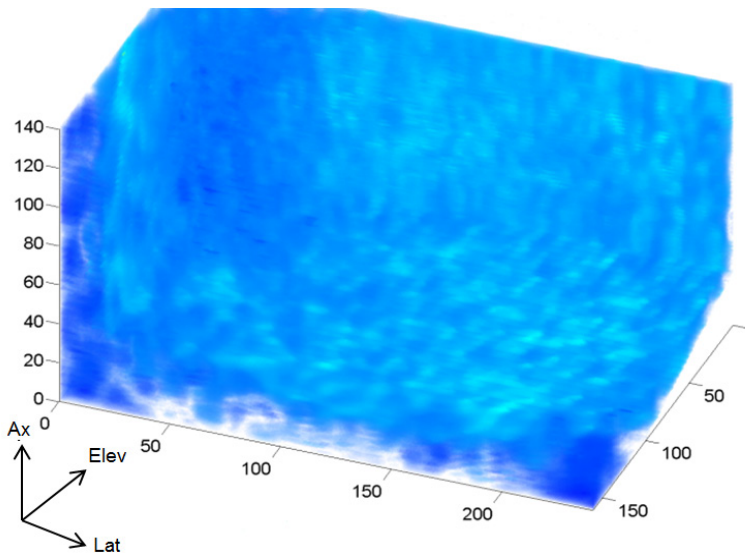

c)

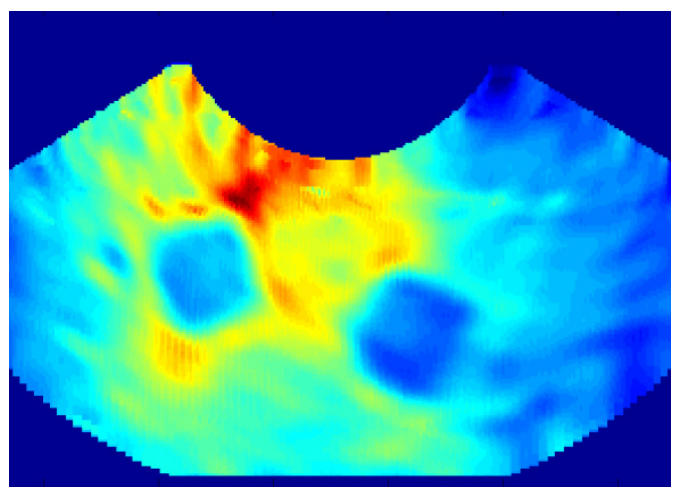

b)

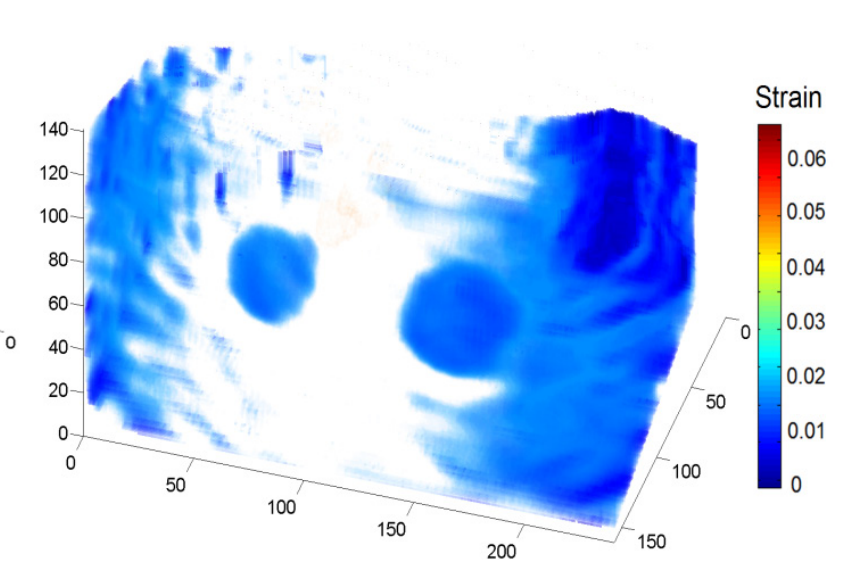

d)

Figure 4.3 2D and 3D Breast phantom imaging: a) Conventional B-mode slice. b) Axial strain elastographic slice. c) Conventional B-mode volume. d) Axial strain elastographic volume. Axes are in pixel units, where 10 pixels equal $2 \mathrm{~mm}$. 
The inclusions can hardly be noticed in the B-mode slice and they were marked with yellow circles as shown in Fig 4.3 a). While in the elastography slice, Fig 4.3 b), no marking was needed and the inclusions can be noticed clearly. Figure $4.3 \mathrm{c}$ ) shows the B-mode volume where no inclusion can be seen due to the similar anatomical features between the inclusion and the surrounding background, which resulted in similar voxel (volumetric pixel) values across the whole volume. Yet, in the accompanying elastographic volume the inclusions can be identified clearly from the soft background tissue. The soft tissue exhibited higher strain voxel values than the stiff inclusions, and a contrast between the two tissue types was apparent. The shown elastographic volume appearance was also enhanced by applying a transparency degree to the volume, in which higher values were eliminated (soft tissue) from the display and only low values were displayed (stiff inclusions). The transparency feature was not effective in the B-mode volume when applied, because of the lack of contrast between the tissue types.

In the generated elastography imaging, two low strain bands appear on both right and left sides of the image/volume. This is because of the sector shape of the used 3D ultrasound probe, which is the main compression device throughout our study. Because of the array shape being sector, the transmitted ultrasound beams are diverging as they propagate through the tissue. As a result, the regions attached to the image borders would exhibit nearly no compression, and the estimated strains would be very low. In addition, the angle of compression was not perfectly perpendicular to the tissue because of the curved surface of the phantom that simulates human breast in the supine position, which resulted in additional non-uniformity of tissue compression. Those low strain regions have similar color representation as the stiff inclusions, because they have similar pixel/voxel values. This effect was not noticed with the linear array experiments, as the ultrasound beams were not diverging, which made the region imaged under the probe experience a uniform axial compression. To avoid the confusion between the real stiff inclusions and the low strain regions, we had to make sure that the inclusions were located in the middle of the image, i.e. directly under the imaging probe. The regions directly below the imaging probe were experiencing the correct amount of axial compression, and the strain contrast between soft and stiff tissue would be correctly emphasized.

\subsubsection{Derived Strains Imaging}

Equations 3.5 were applied on the axial normal and shear strain elastography, to construct the additional three derived strains mentioned earlier. Figure, 4.4 shows the axial, first principal 
strain, Von Mises and maximum shear elastographic volumes. The figure demonstrates volumetric views for a single inclusion to emphasize the difference. The normal axial strain and axial shear strain volumes were first constructed from the RF data, then the derived strain were calculated on a pixel by pixel basis for each slice in the volume.

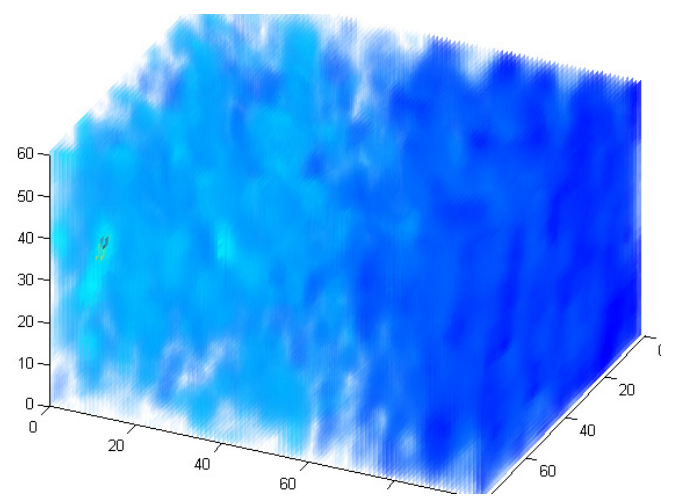

a) B-Mode volume

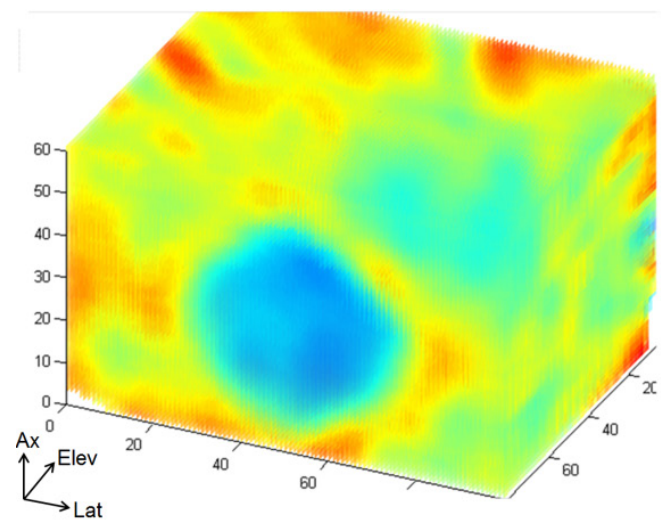

b) Axial strain

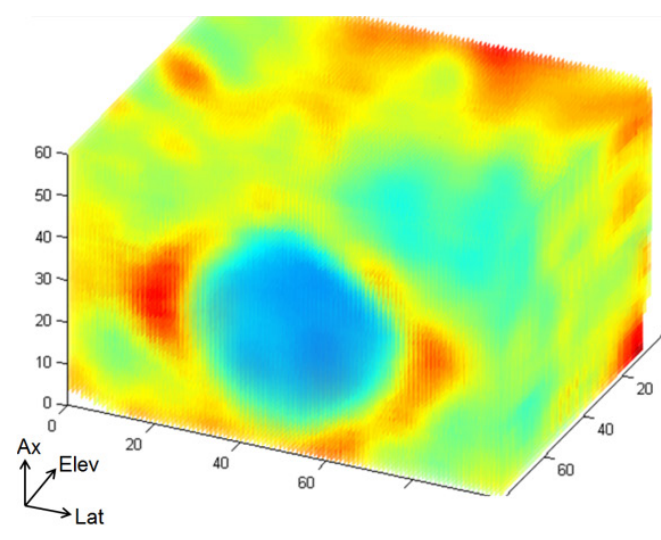

d) Von Mises strain

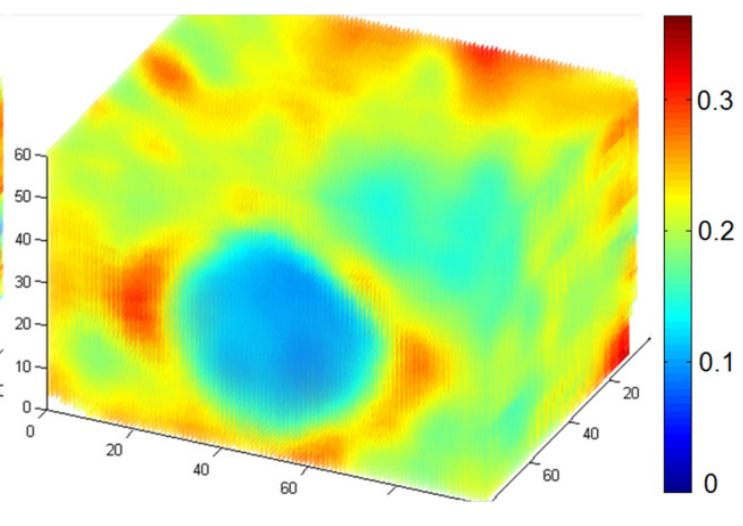

c) First principal strain

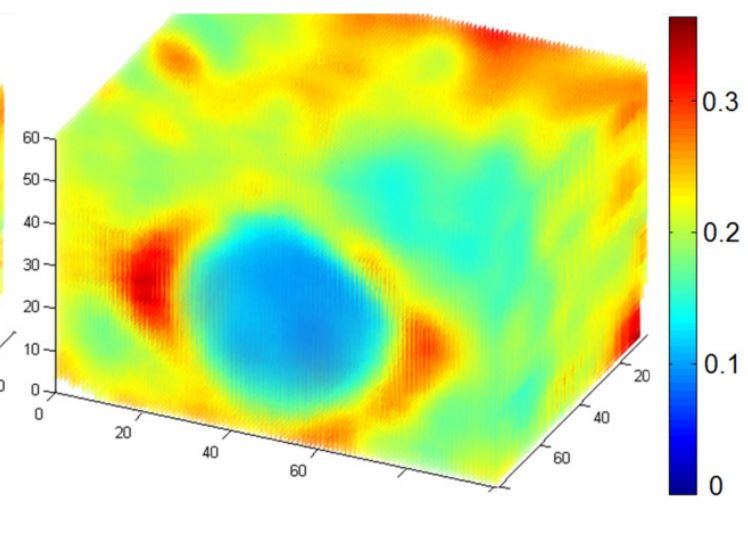

e) Maximum shear strain

Figure 4.4 3D Breast phantom imaging using different volumetric strain types. Color bar represents normalized strain values. Axes are in pixel units, where 10 pixels equal $2 \mathrm{~mm}$. 
The strain values within each volumetric strain type were normalized with respect to the other strain types. The normalization was necessary to have a fair comparison between the different strains. Without performing cross normalization each volume would have a different color map, and the inclusion's boundary enhancement would not be clearly and fairly emphasized. The cross normalization was achieved by collecting columns of the different strains images together in a single matrix, and then this matrix was column wise normalized. The new values were returned back to their original locations in the corresponding volume, so that the four elastographic volumes would be column wise normalized together.

As in the case of the 2D elastography, first principal, Von Mises and maximum shear elastographic volumes emphasis the inclusion's boundary. The maximum shear strain volume demonstrates the most evident boundary enhancement effect. Figure 4.5 presents the normalized strain profiles for each strain type through the inclusion along the axial direction. The figure shows that the difference in amplitude between the inclusion's strain value and the surrounding soft background is largest in the maximum shear profile. The high peaks of the strain profiles could be considered as marking points for the inclusion's bounds. This marking points can be used to better segment the inclusion and calculate its volume, as will be shown in the next subsection. The figure also shows that the Von Mises strain, then the first principal strain follows the maximum shear strain in the edge enhancement degree.

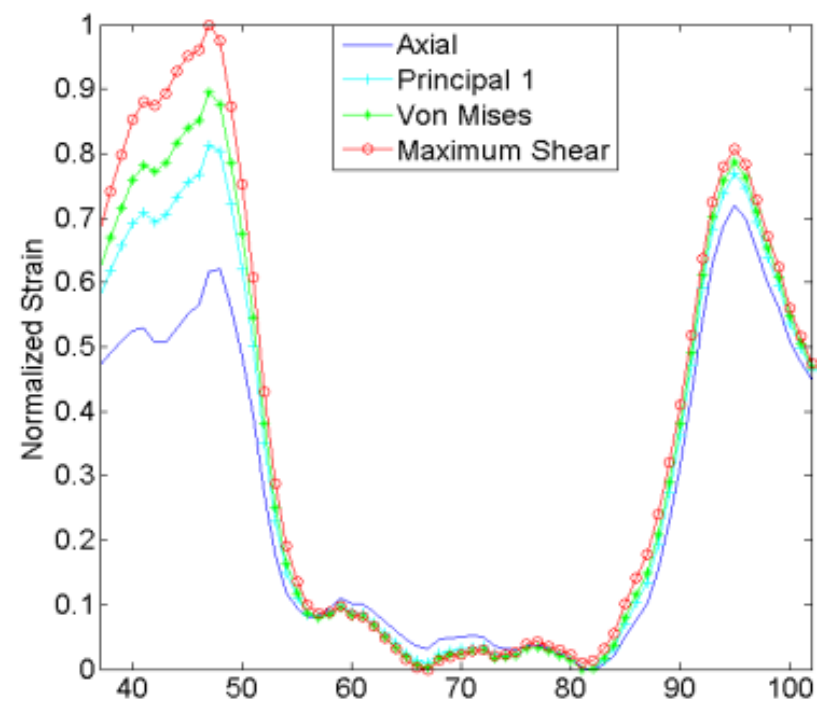

Figure 4.5 Strain profiles across breast phantom's inclusion. Horizontal axes are in pixel units, where 10 pixels equal $2 \mathrm{~mm}$. 
The theory behind the edge enhancement can be elucidated by the fact that the last three strains were derived originally from normal axial strain and axial shear strains, so both strains play their roles in the elasticity imaging process. An important characteristic of this boundary enhancement feature is that it has occurred at an angle relative to the axial compression direction, not along or normal to it. This is a fundamental property of the shear strain, and that angle depends on the amount of shear strain exhibited by the stiff tissue. The axial strain provides contrast in the direction of the mechanical compression, while the axial shear provides contrast with an angle relative to the direction of the mechanical compression, and that angle depends on the amount of exhibited shear strain. Shear slip mechanism at the interface between dissimilar materials was the main factor for the potential boundary enhancement. Those derived strains show the inclusions and inclusion-background border with different degrees of clarity, according to the weights assigned to the normal axial strain and axial shear strain for each strain type's equation.

\subsection{Quantitative measures}

\subsubsection{Relative Stiffness}

The relative stiffness of the inclusion is a quantity that tells how many times the stiff inclusion is stiffer than the surrounding soft tissue. The relative stiffness between the stiff inclusion and the soft background can be expressed mainly by the axial strain elastograms, as it reflects directly the amount of mechanical deformation exhibited by the tissue under compression. Whilst, the other derived strain elastograms do not embrace this property, because they incorporate the axial shear strain that reflects the sliding angle change between tissue layers. As a result, relative normal axial strain calculations are the main and correct measure for the relative stiffness of the stiff inclusion.

In elastographic studies, the relative stiffness is defined as the ratio between the soft tissue and the stiff inclusion strain values. The method by which the region of strain calculations is determined is manual selection, i.e. through manual drawing of rectangles containing the designated regions. Each selected rectangular region contains a number of pixels, and their mean value is computed and stored for the ratio calculation. In 2D free hand elastography, we obtain a single value for each frame, and we can calculate the ratio for a number of pre-specified frames, to obtain a mean value with the associated standard deviation. While, in the 3D mechanical compression 
elastography we obtain a number of values equal to the number of slices in the volume containing the stiff inclusion, and a mean ratio value with standard deviation is also attainable in this case.

In both cases, the method of region selection is shown in Fig. 4.6. First, the selection of the stiff inclusion(s) is done, then the selection of the soft tissue follows under the condition that the selection is performed at nearly the same horizontal level, i.e. imaging depth. This approach of selection ensures that both regions were nearly subjected to the same compression level, and thus the strains induced in both regions would reflect the tissue behavior at that specific compression level. Choosing regions at different depths would result in inaccurate measurements.

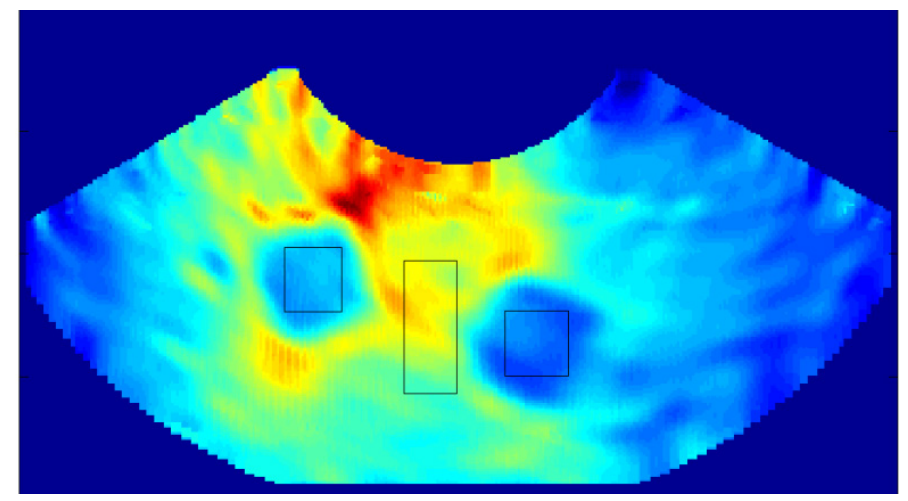

Figure 4.6 Regions selected for quantitative analysis.

Equation 3.9 was applied for both the 2D free hand and 3D mechanical compression cases, based on the same number of frames and slices. The resultant values were the observed relative stiffness for the phantom's inclusion. Considering the general concept of contrast transfer efficiency (CTE) [107, 108], which puts a limitation on the observed relative stiffness of $85 \%$ of the true relative stiffness, the observed relative stiffness was corrected accordingly by this percentage $(85 \%)$. The resultant corrected relative stiffness values are shown in Table 4.1:

Table 4.1 Relative stiffness calculations.

\begin{tabular}{c|c|c}
\hline Relative Stiffness & Value & Mean Error \\
\hline Phantom Specifications & 3 & \\
\hline 2D Freehand & $2.807 \pm 0.384$ & -0.193 \\
\hline 3D Mechanical Compression & $3.026 \pm 0.218$ & 0.026 \\
\hline
\end{tabular}


Observing that the phantom's manufacturer specification states that the inclusions are approximately three times stiffer than the surrounding soft tissue, the error of measurement can be computed. From the table, the error in calculating the relative stiffness is lower using the mechanical compression technique, as the compression in this case is almost uniform through volumetric slices and no vibrations or lateral movements occur during compression. The 2D freehand compression technique suffers from hand vibrations and instabilities during acquisition, which resulted in some inaccuracies in the relative stiffness computations. These observations agree well with previous similar studies $[45,46,109]$.

\subsubsection{Inclusion Volume Calculation}

Another quantitative measure that was specifically calculated for the volumetric elastograms is the inclusion's volume. The main purpose of the volume calculation was to assess the geometric accuracy of the elastographic volumes. The actual inclusion's volume was calculated by measuring the inclusion's diameter from the B-mode images. This was a valid method as the inclusion's diameters were pre-determined and listed by the phantom manufacturer, so a single measurement of the diameter was enough to know its actual volume.

As mentioned earlier, the volume was calculated as the summation of individual cross sectional areas of the inclusion in each slice through the designated volume. This was done after performing scan conversion in the elevational direction for the strain slices, and constructing the final correct volumes. In each volume slice, the inclusion was segmented manually and its area was calculated. During segmentation, strain artifacts that appear regularly in the lateral directions were kept away from the selected areas. Using this method, the calculated inclusion volumes for each constructed strain type was compared to the actual inclusion volume. The calculated volumes along with the associated errors are demonstrated in Table 4.2. 
Table 4.2 Inclusion volume calculations

\begin{tabular}{c|c|c|c}
\hline & Volume $\left(\mathrm{mm}^{3}\right)$ & Error & Estimated Radius $(\mathrm{mm})$ \\
\hline Actual Volume & 381.704 & & 4.50 \\
\hline Axial Strain & 352.076 & $-7.76 \%$ & 4.38 \\
\hline Principal 1 & 367.297 & $-3.77 \%$ & 4.44 \\
\hline Maximum Shear & 382.807 & $0.29 \%$ & 4.50 \\
\hline Von Mises & 394.821 & $3.44 \%$ & 4.55 \\
\hline
\end{tabular}

The elastographic calculated volumes were verified to be in the range the phantom's manufacturer specifications. The volume and radii estimates listed in the table above, show that the maximum shear volume provides the best inclusion volume preservation. One reason for that is the ease of determining the inclusion's boundary during the segmentation process, in which the area where specified more accurately than the other volumetric strains.

\subsection{Imaging Quality Assessment}

Imaging quality measures were used to assess the resultant elastographic images and volumes. Signal to noise ratio (SNR) and contrast to noise ratio (CNR) values were calculated in decibels for the $2 \mathrm{D}$ and $3 \mathrm{D}$ elastography methods, using equations 3.7 and 3.8 respectively. The resultant values agreed well with similar previous studies $[45,46,53,55,101,110-112]$ and prove that the quality of the used strains imaging techniques is acceptable.

\subsubsection{Elastography Frames}

The SNR and CNR were calculated for the four elastographic image types, as shown in Fig. 4.7. The presented values are the mean values of the quality parameters along with their corresponding standard deviations. The parameters were calculated using the selected regions of interest (ROI's) for the uniform soft background and the stiff inclusion for each frame. This selection process was repeated for a number of 15 frames. 


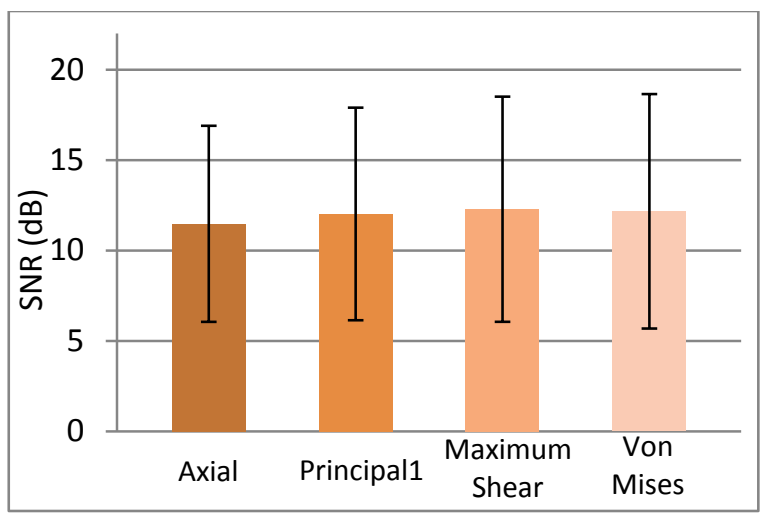

a)

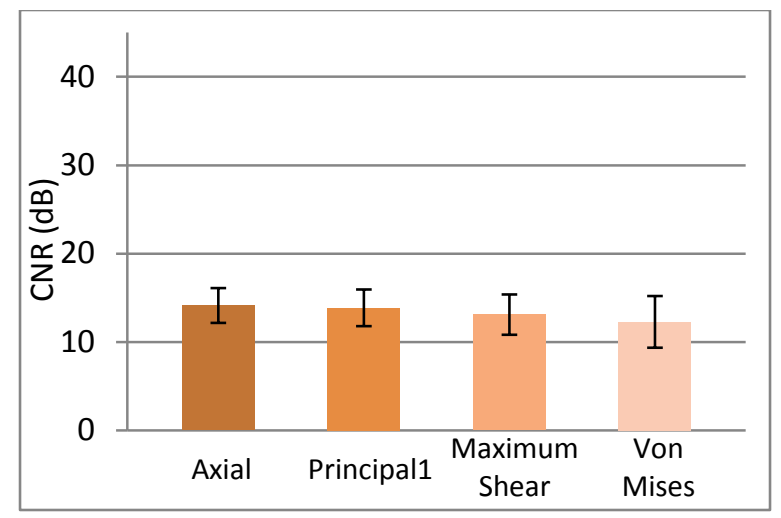

b)

Figure 4.7 2D elastography imaging quality assessment: a) SNR. b) CNR.

Figure 4.7 a) shows that the mean SNR is approximately the same between the four types of elastograms, with a minor increase in the derived strain types. The first principal strain mean SNR was higher than the axial strain with about $0.5 \mathrm{~dB}$, maximum shear was higher with $0.8 \mathrm{~dB}$ and the Von Mises was higher with $0.7 \mathrm{~dB}$. The SNR standard deviation values were nearly the same in the four cases. The CNR for the four types of elastograms is demonstrated in Fig $4.7 \mathrm{~b}$ ). The large scale used in the figure was to emphasize the difference between 2D and 3D imaging illustrated in Fig 4.8 in the next subsection. The axial and first principal strains had nearly the same mean CNR, yet the maximum shear and Von Mises showed a slightly decreasing trend in the CNR. The maximum shear mean CNR was lower with $1.0 \mathrm{~dB}$ than the axial strain, and the Von Mises was lower with 1.8 dB. The CNR standard deviation values were slightly higher in the maximum shear and Von Mises than the axial and first principal strains, as a result the maximum CNR value in the four cases were nearly the same.

\subsubsection{Elastography Slices}

The SNR and CNR were also calculated for the four elastographic volumes presented in Fig 4.4. In this case, the ROI's for the soft background and the stiff inclusion were selected in the volumes slices. The mean and standard deviation values were calculated for a number of 32 slices constituting the elastographic volumes. A comparison of SNR and CNR using different strain imaging types is illustrated in Fig 4.8. 
Observing the SNR value for each volume type in Fig 4.8 a), we notice a slight increase in the mean SNR of the three derived strain volumes over the axial strain volume with about $0.8 \mathrm{~dB}$. Furthermore, the standard deviation of SNR's for the four strains have nearly the same limits. Consequently, the three derived strains exhibit nearly the same level of SNR.

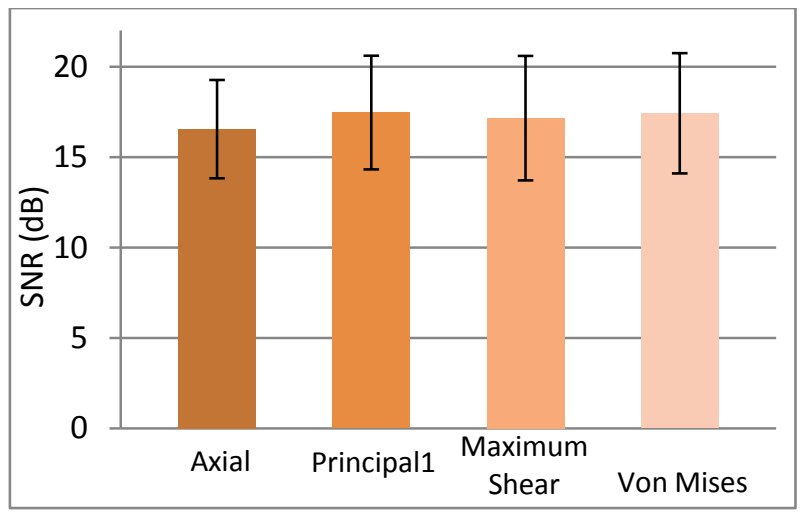

a)

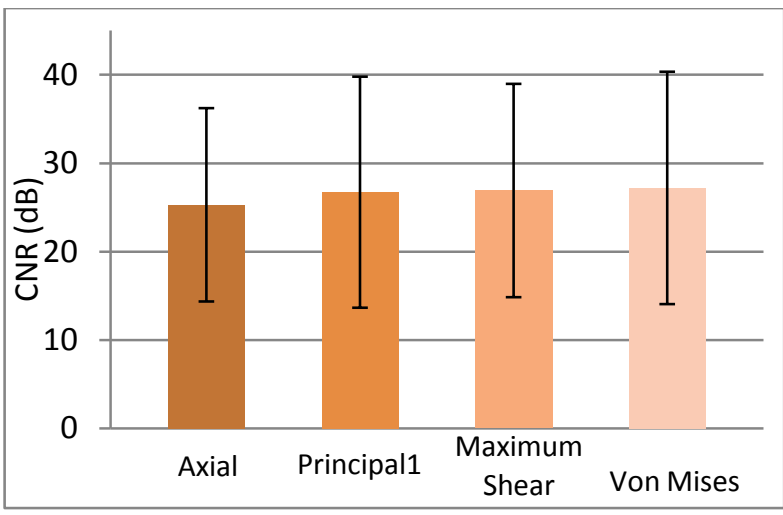

b)

Figure 4.8 3D elastography imaging quality assessment: a) SNR. b) CNR.

Figure $4.8 \mathrm{~b}$ ) presents the relationship between the four volumetric strains CNR. We noticed that the derived strains provide a mean CNR values better than the axial strain with about $1.6 \mathrm{~dB}$. In addition, the CNR standard deviations for the derived strain volumes are higher than the axial strain volume with approximately $1.8 \mathrm{~dB}$. Note that the SNR and CNR values are very close in the four cases, with some improvement in the three derived strain volumes.

A general observation on the SNR and CNR values in both 2D and 3D elastography imaging methods, is that the values obtained in the $3 \mathrm{D}$ case is significantly higher than those in $2 \mathrm{D}$ case. The CNR was particularly enhanced in the 3D case with an approximate factor of 2 . An improvement of about $5 \mathrm{~dB}$ in the SNR and over $10 \mathrm{~dB}$ in the CNR was observed. This effect can be explained by the following reasons:

1. The compression method: Mechanical compression provides a steady method, while freehand suffers from shaking vibrations and instability of the compression level. Freehand method also relies heavily on the user skills and experience.

2. The acquisition method: Acquiring complete volumes at fixed pre- and post-compression levels provides a uniform application of strain over the entire volume. 


\subsection{Summary}

This chapter demonstrates the in vitro breast phantom study results. A number of significant results were obtained, including the successful construction of 3D elastographic volumes, and the introduction of new types of strain imaging in 2D and 3D. The results show that the new types, especially maximum shear strain elastography, would provide a significant visual improvement to breast masses imaging. Inclusion volume calculation accuracy using this type outperforms the accuracy using the common axial strain imaging. In addition, this feature can be used to better segment the stiff lesions for an improved visualization and characterization. The new strain imaging types is not expected to result in a loss of signal or details. This was shown to be true by calculating the signal to noise ratios and contrast to noise ratios for the presented strains.

The main specific aims of this study were:

1. Prove that $3 \mathrm{D}$ strain imaging is advantageous over $2 \mathrm{D}$ imaging in both visual and quantitative aspects. The view of the stiff inclusions inside soft tissue in 3D was very informative and attractive.

2. The in lab phantom study provided a means to tune the elastography algorithm so as to produce the optimum imaging quality, through trial and error. Imaging parameters were continuously adjusted till the best volumes were reached. This was necessary before proceeding to the clinical in vivo study. The main optimized imaging parameters was found to be as follows:

- Application of about $1 \%$ axial tissue compression.

- Central imaging frequency of $9 \mathrm{MHz}$.

- Imaging depth of 5 to $8 \mathrm{~cm}$.

- RF gain of $65 \%$ of the full gain.

- Temporal window length of $1.6 \mathrm{~mm}$.

- Temporal window overlap of $75 \%$.

- Cubic spline sample interpolation ratio of 20:1 on individual windows.

- Cross correlation time lags of 5 samples only was enough. This was beneficial in speeding up the estimation process.

- Displacement cubic spline smoothing weight of 0.3.

- Median filter of 12X10 pixels was good for most cases.

- Scan conversion interpolation window of $3 \mathrm{X} 3$ pixels. 
- Display 3D volume using 2D- texturing, showing details from inside whenever possible, with a transparency ratio of 0.2 .

3. A better understanding of strain images and how to interpret them. The knowledge of the common image artifacts facilitated the differentiation between low strain areas and stiff masses in the in vivo study, as will be shown in the next chapter. 


\section{CHAPTER 5}

\section{IN VIVO CLINICAL STUDY}

This chapter demonstrates the pilot elastography study conducted in vivo on a number of ten volunteered patients. The study tried to examine human breast masses detectability using elastography, and attempted to characterize them accordingly. 3D elastography imaging results are illustrated throughout this chapter. The new method of breast mass classification was examined and the classification outcomes were verified with biopsy diagnosis. Moreover, derived strains elastography imaging results are demonstrated and discussed. The recruitment policies of the patients were also mentioned for reporting completeness. The results of this study was published as a technical journal paper [113].

\subsection{Study Aims and Policies}

The main goal of conducting this clinical study was to characterize the biomechanical behavior of breast masses using elastography under different loads, or compression levels. While this main goal is quite general, the study specific aims were to address the following points:

1. Construct 3D elastography for breast masses, either detected masses through mammography/conventional ultrasound, or detected through palpation.

2. Evaluate 3D elastography as a new concept in clinical breast care.

3. Assess the diagnostic quality of the produced elastography images and volumes.

4. Classify breast masses through a novel characterization method based on a nonlinear tissue model.

5. Verify and compare the classification results with the invasive biopsy diagnosis.

6. Apply segmentation algorithms on the in vivo elastographic data and estimate their sizes.

A number of policies were followed before and during the execution of the study in the Betty Puskar breast care center, West Virginia University. First, an institutional review board (IRB) approval was issued before performing the study to monitor the ethical aspects, as female human subjects were involved. Second, the involvement of each patient had to follow a patient's recruitment policy. This policy mandates that to qualify a patient for the inclusion in the study, she should have known masses that were detected by either two methods: 
1. Mammography imaging showing the presence of a suspected stiff mass inside the breast tissue.

2. Palpation: If a mass was palpable and its presence was confirmed by conventional ultrasound imaging and/or mammography.

In addition, before commencing elastography scanning, each patient must give an informed consent, confirming her complete awareness and approval of the study and its nature including the knowledge of the benefits and possible risks or discomforts.

Patient's confidentiality policy was mandating no personal information to be used in the scanning images, reporting, results analysis or publications. Mammography images were deidentified and given random numbers to be associated with the corresponding ultrasound and elastography images. After the completion of scanning of each patient, a short survey was asked for completion by the patient. The survey was questioning the patient about any discomforts experienced and also the time involved, as well as her likelihood to repeat the procedure again, if offered in the future.

The acquired RF data were then processed offline on a separate workstation. Resultant elastographic images and volumes were compared with conventional ultrasound and mammography to confirm the location of the aimed masses. Mass classification outcome, being benign or malignant, were confirmed with biopsy. The final patient report was prepared by both the biomedical ultrasound lab and Betty Puskar breast care center, and given a random reference number for identification.

\subsection{D Breast Mass Strain Imaging}

A number of ten patients were examined using mammography, conventional ultrasound and 3D elastography. One patient was excluded from the analysis, as the breast mass was located on the edge of the breast, and it was very difficult to acquire useful and analyzable elasticity images for this case. Another patient had two breast masses; therefore the total number of examined masses was 10. The examined masses were divided into two categories, malignant and benign, wherein each category consists of five masses. The diagnosis was confirmed with the biopsy pathologic results, which is currently the gold standard for breast cancer diagnosis.

The modified mammography paddle was used to hold the 3D ultrasound probe. The level of tissue compression was delivered and controlled by the mammography station. All the scanned 
volumes consisted of a total number of 40 slices, with a total field of view of 58.5 degrees. For each case, the stiff mass was first located and marked under conventional B-mode ultrasound by a radiology technician. The presence of the mass was confirmed with the guidance of an experienced radiology doctor. The highest compression level (F3) was applied first on the breast tissue, then the lower levels, F2 and F1. This was to ensure stability of the breast tissue under compression. Because of the curvy shape of the breast tissue, starting with the lower compression levels could cause the tissue to slip under the stage as the compression level increases. The 3D elastography algorithm was then applied on the acquired volumetric RF data as described in chapter 3, with the tuned imaging parameters as enumerated in chapter 4.

Imaging session as a whole took about 15 minutes for each patient, which was considered to be acceptable. Most of this time was to properly position the patient on the stage and align the ultrasound probe directly over the breast mass. The patient had to stay still and take shallow breaths only for approximately one minute or less. This hold time was necessary to minimize motion artifacts, and it was only required during the application of the three pre-compression levels and acquisition of the RF data.

\subsubsection{Benign cases}

Benign masses are usually harder than the surrounding soft tissue with an amount of 1.5 to 4.5 times. This mild relative stiffness make visualizing benign masses in $2 \mathrm{D}$ strain images a difficult task, as the contrast is poor for such masses. Yet, using 3D elastography, the visualization of benign masses was improved.

Figure 5.1 demonstrates a benign case for a 40 years old patient with a palpable mass, imaged using B-mode ultrasound, mammography, 2D and 3D elastography, respectively. Ultrasound imaging depth was $8 \mathrm{~cm}$ 's. The region of interest used for elastography images was marked on the B-mode images with a yellow rectangle, while the stiff masses were labeled mass 1 and mass 2 . Mammography showed that the patient had multiple well-circumscribed masses in the right breast. The palpable mass at 8:00 was reported to be increasing in size by the patient. This corresponds to mass number 1 on the images.

The strain contrast between the stiff masses and the surrounding soft tissue was clear in the elastography volume. During scanning of this case, mass 1 was the primary target of the study for both imaging and biopsy. However, while processing the elastography images, mass 2 appeared in the elastograms, especially in the 3D elastogram. The location of the other mass was compared and 


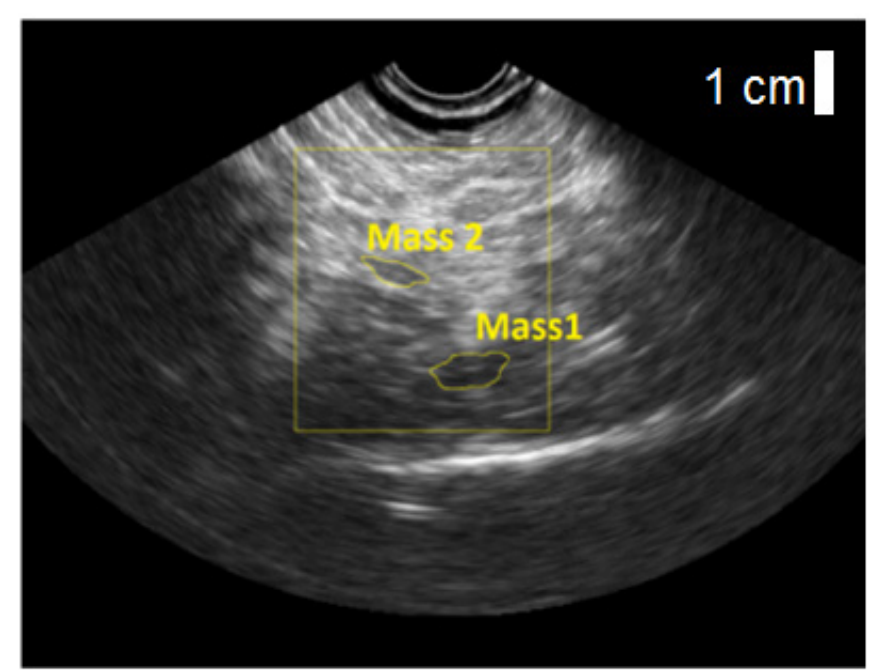

a)

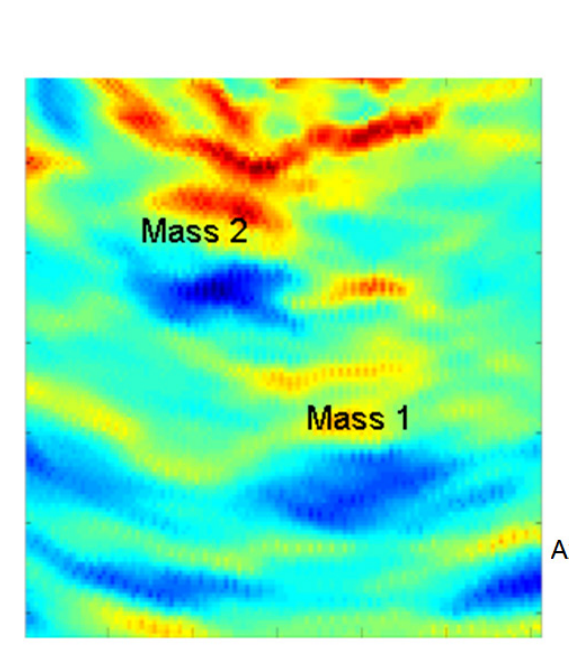

c)

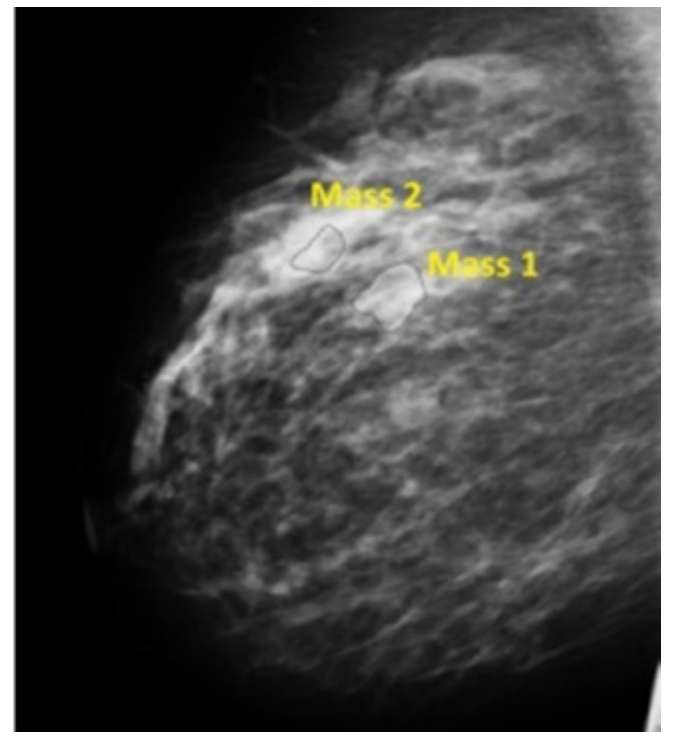

b)

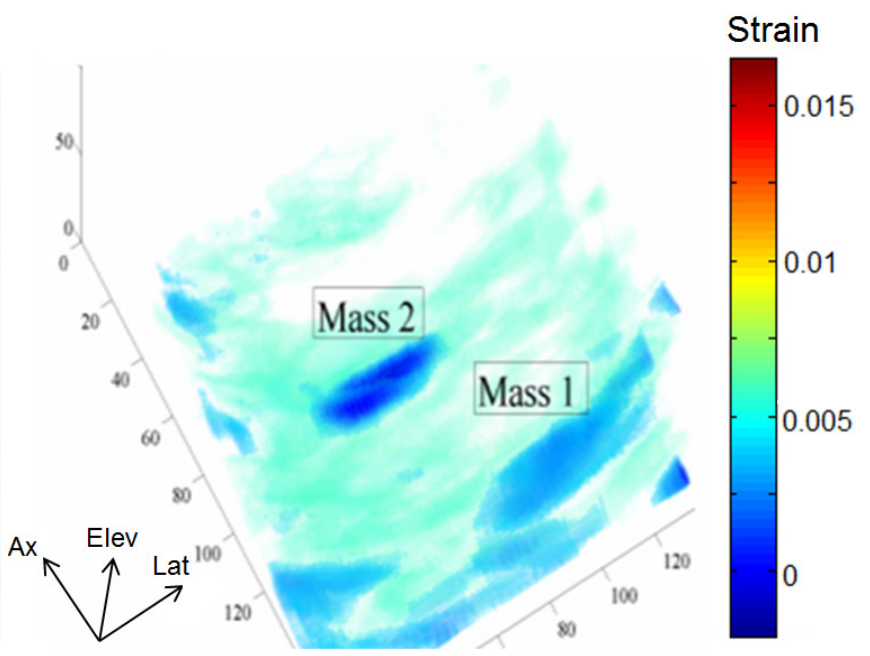

d)

Figure 5.1 Case number 1, benign breast mass: a) conventional ultrasound, b)

mammography, c) elastography image d) elastography volume. Axes are in pixel units, where 10 pixels equal $4 \mathrm{~mm}$.

confirmed with mammography images, which showed a vague appearance of another mass, Fig 5.1 b). 3D elastography was also superior over conventional B-mode imaging that showed an unclear hypoechoic pattern at the mass location, as marked in Fig 5.1 a). These observations proved the potential of using elastography for the detection and visualization of breast masses. The 3D method exhibited an additional advantage of displaying the stiff masses shape in three dimensional space, which gave more information about the relative size and shape of the mass with respect to the 


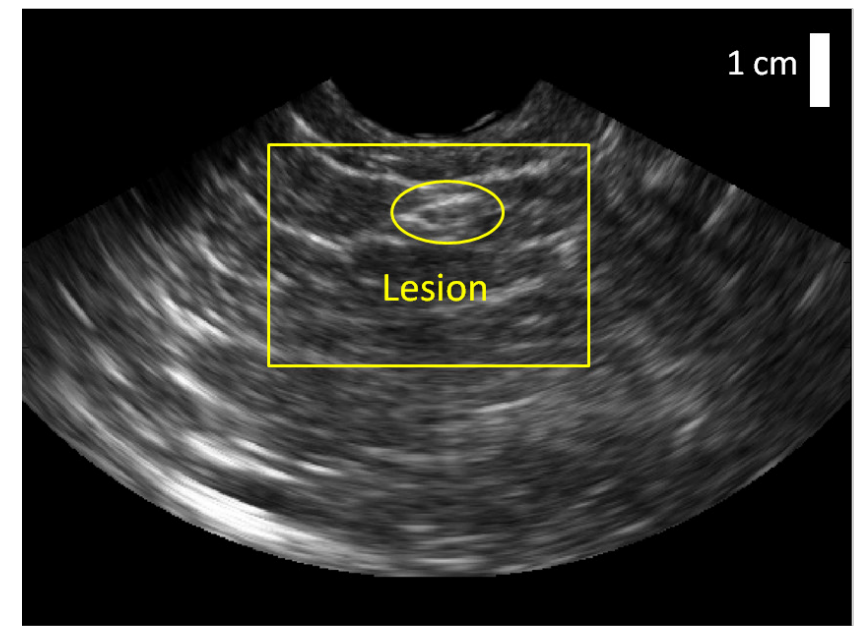

a)

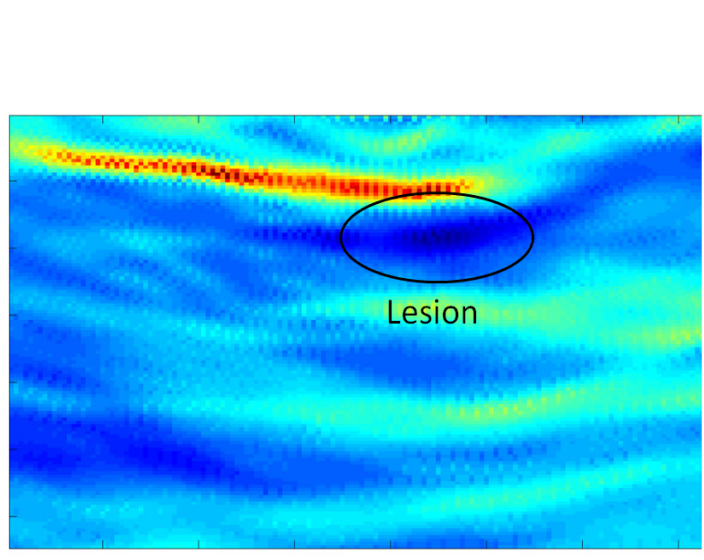

c)

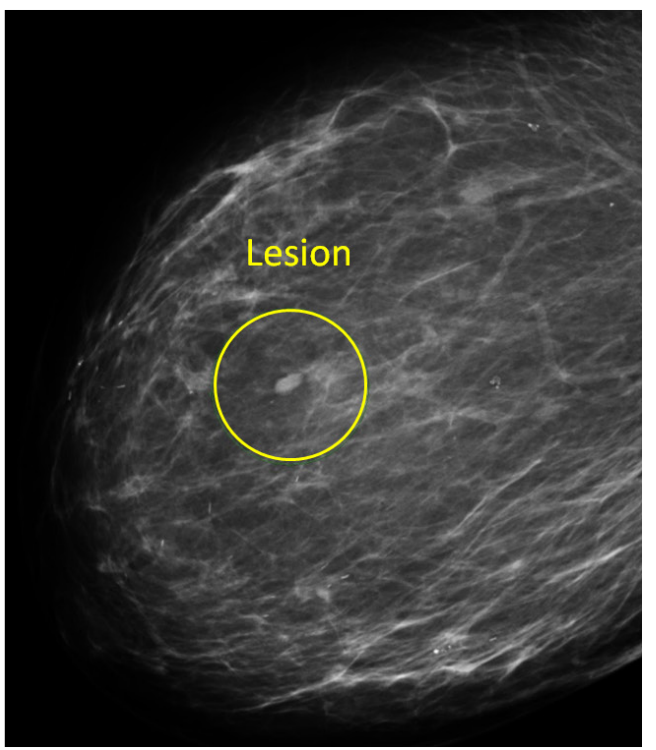

b)

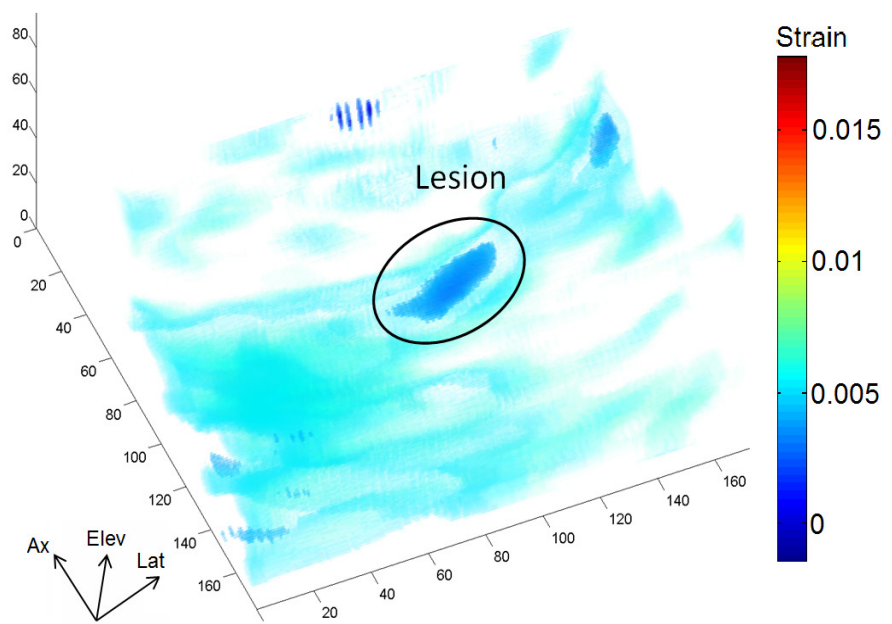

d)

Figure 5.2 Case number 5, benign breast mass: a) conventional ultrasound, b) mammography, c) elastography image d) elastography volume. Axes are in pixel units, where 10 pixels equal $2 \mathrm{~mm}$.

surrounding tissue. In 3D elastography, the outline of the masses was obvious and they had an ellipsoidal shape. In addition, the masses appeared as blue and dark blue formations, while the soft background had a cyan color. The last observation would indicate some characteristics about the stiff mass and suggest benignly, as will be discussed later in this chapter.

Figure 5.2 demonstrates another benign case for a 55 years old patient with a non palpable mass, imaged using B-mode ultrasound, mammography, 2D and 3D elastography, respectively. Ultrasound imaging depth was $6 \mathrm{~cm}$ 's. The region of interest on B-mode was also marked with a yellow rectangle, while the stiff mass was labeled as 'lesion' on the images. Mammography showed 
that the patient had an irregular mass at 10:00 in the right breast. On ultrasound, there was an irregular mass with angular margins in the right breast.

As in the previously shown case, the strain contrast between the stiff mass and the surrounding soft tissue was clear in the elastography volume. In 3D elastography, the outline of the mass was obvious but it had an irregular shape. In addition, the mass appeared as a dark blue structure, while the soft background had a cyan color. This again indicates a benign mass, because of the relatively low contrast between the mass and the soft tissue.

\subsubsection{Malignant cases}

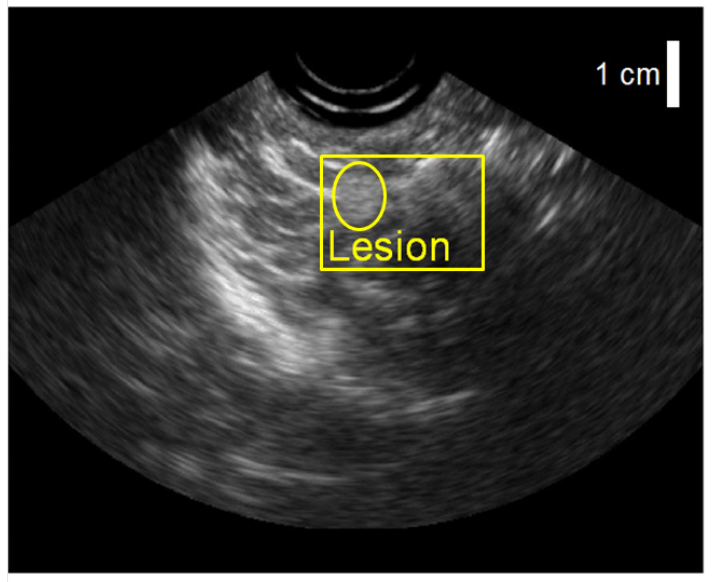

a)

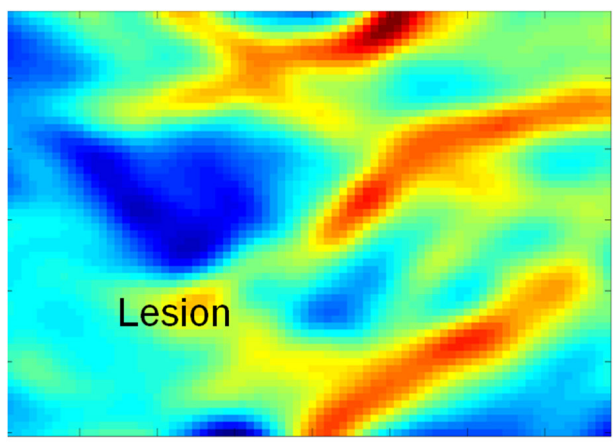

c)

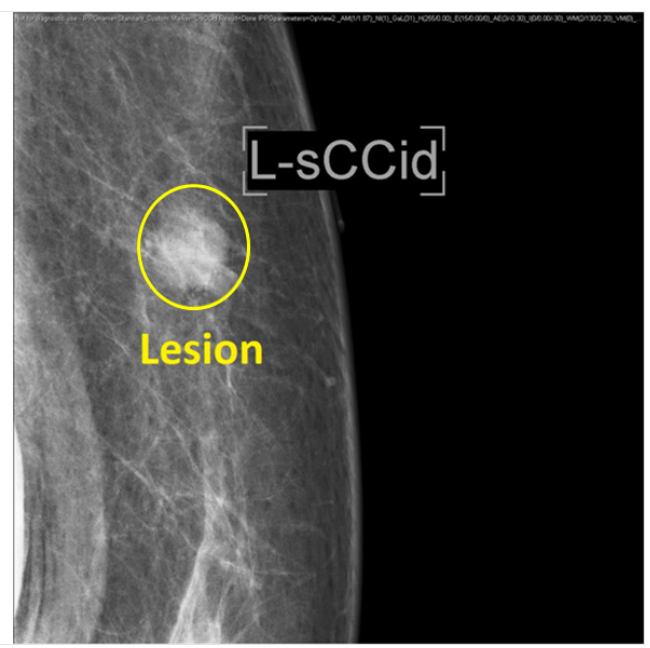

b)

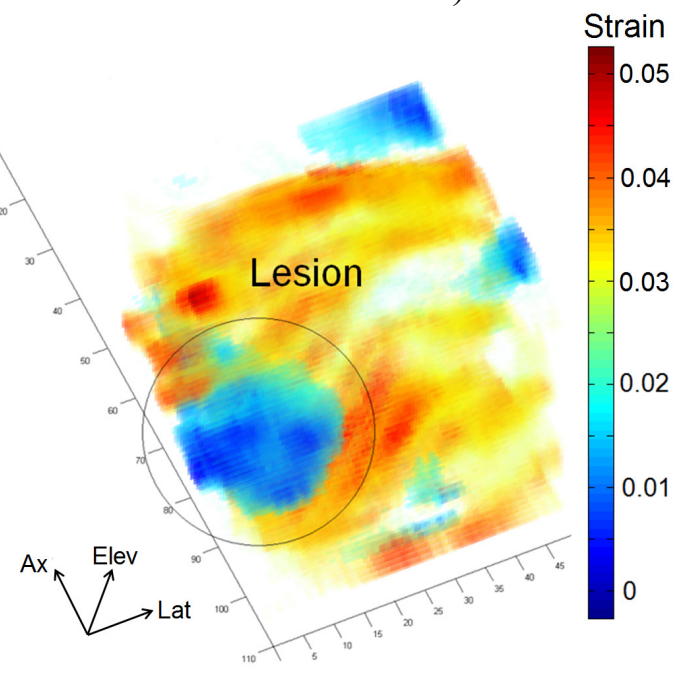

d)

Figure 5.3 Case number 2, malignant breast mass: a) conventional ultrasound, b) mammography, c) elastography image d) elastography volume. Axes are in pixel units, where 10 pixels equal $5 \mathrm{~mm}$. 
Figure 5.3 shows a malignant case for a 46 years old patient with a palpable mass, imaged using B-mode ultrasound, mammography, 2D and 3D elastography. The used ultrasound imaging depth was $6 \mathrm{~cm}$ 's. The ROI on B-mode was also marked with a yellow rectangle, while the stiff mass was labeled as 'lesion' on all images. Mammography showed that the patient had a solid mass at 11:00 in the left breast. On ultrasound, there was a hypo echoic mass at the center of the image.

In this malignant case, the strain contrast between the stiff mass and the surrounding soft tissue was significant in the elastography image, and the mass was so distinct from the soft tissue. In $3 \mathrm{D}$ elastography, the outline of the mass was evident and it had an elongated spherical shape. In addition, the stiff mass appeared as a blue structure, while the soft background had an orange to yellow colors. The other blue regions in the shown volume corresponds to stray low-strain regions and do not correspond to anatomical masses. This high contrast in strain between soft and stiff tissue suggested a mass with large relative hardness, indicating a malignant mass. This was in agreement with the biopsy results that revealed a mass with invasive ductal carcinoma.

Figure 5.4 shows another malignant case for a 46 years old patient with a palpable mass, imaged using the four modalities. The used ultrasound imaging depth was also $6 \mathrm{~cm}$ 's. The yellow rectangle on the ultrasound image represents the used ROI used for obtaining elastographic images, while the stiff mass was labeled as 'Mass' on all images. Mammography imaging showed that the patient had an oval mass with partially obscured margins in the left breast at 2:00. On ultrasound images, a clear hypoechoic, and solid mass with no distinct boundaries was found in the left breast.

As in the case number 2, the strain contrast between the stiff mass and the surrounding soft tissue was significant in the elastography image, and the mass was distinct from the surrounding soft tissue. The targeted mass was plain in the elastogram with clear boundaries. Some strain artifacts were observed on the right edge of the mass. This was because the mass was located close to the right edge of the imaging plane. In 3D elastography, the outline of the mass was obvious and it had an elongated ellipsoid shape. In addition, the stiff mass appeared as a blue structure, while the soft background had an orange to yellow colors. This high contrast in strain between soft and stiff tissue suggested a mass with large relative hardness, indicating that the mass was malignant. This was in agreement with the biopsy results that revealed a mass with high grade invasive ductal carcinoma. 


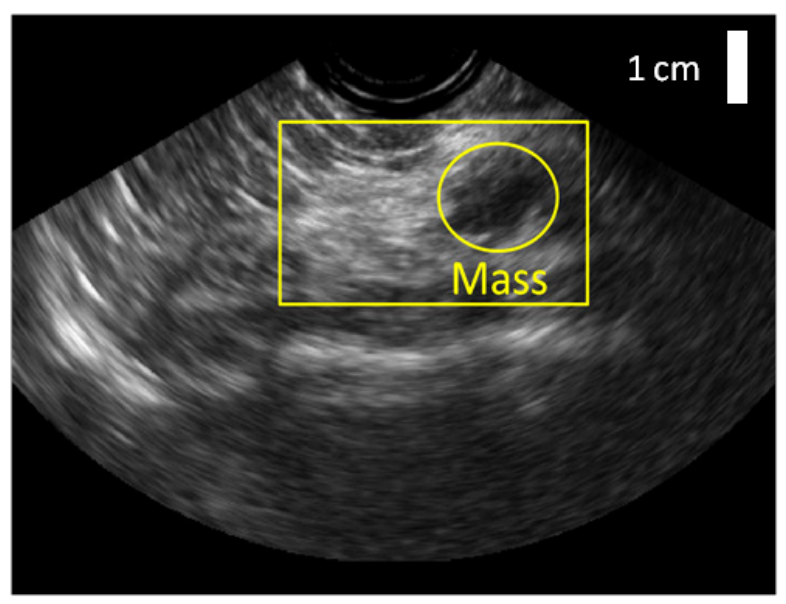

a)

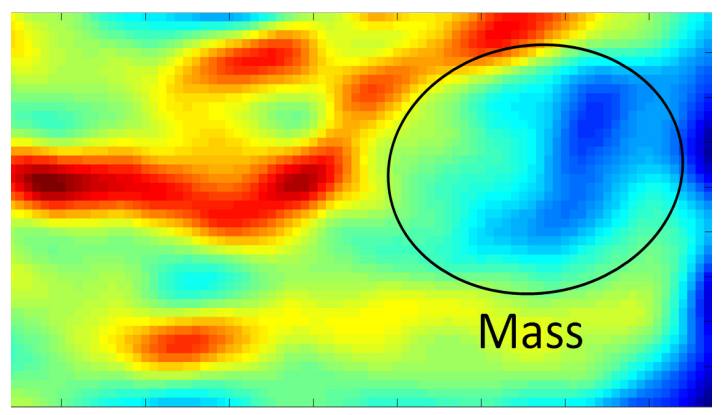

c)

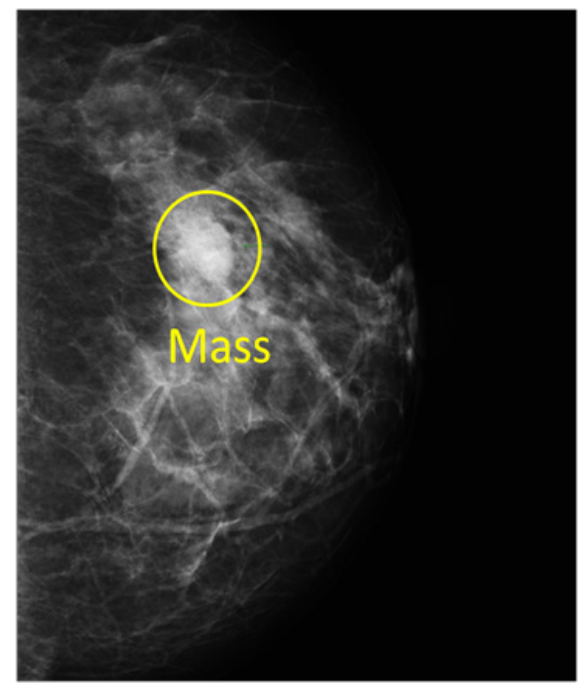

b)

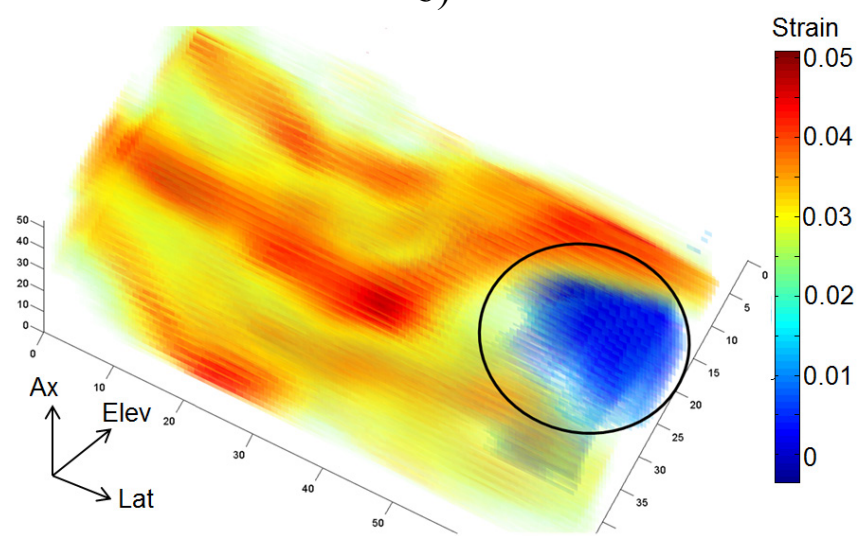

d)

Figure 5.4 Case number 8, malignant breast mass: a) conventional ultrasound, b) mammography, c) elastography image d) elastography volume. Axes are in pixel units, where 10 pixels equal $4 \mathrm{~mm}$.

\subsection{Mass Classification}

\subsubsection{Addressing Tissue Nonlinearity}

In order to implement our new nonlinear classification method, the 3D elastograms were constructed for three pre-compression levels; 2, 3, and $4 \mathrm{kgf}$. The effect of mass nonlinearity was initially observed on the constructed images contrast, as shown in Fig. 5.5. The figure shows elastograms, with a fixed color map for benign and malignant masses under different precompression levels. The fixed color map reveals the change of the mass's strain contrast with the increasingly compression level, wherein the contrast for the benign case was nearly the same at the 
three levels, while the strain contrast was higher for the malignant mass. This strain contrast effect reflects the nonlinear biomechanical effect of the considered masses. Compared to the surrounding healthy tissue, the benign mass's strain was not changed, while the malignant mass's strain was decreased with the increased load.

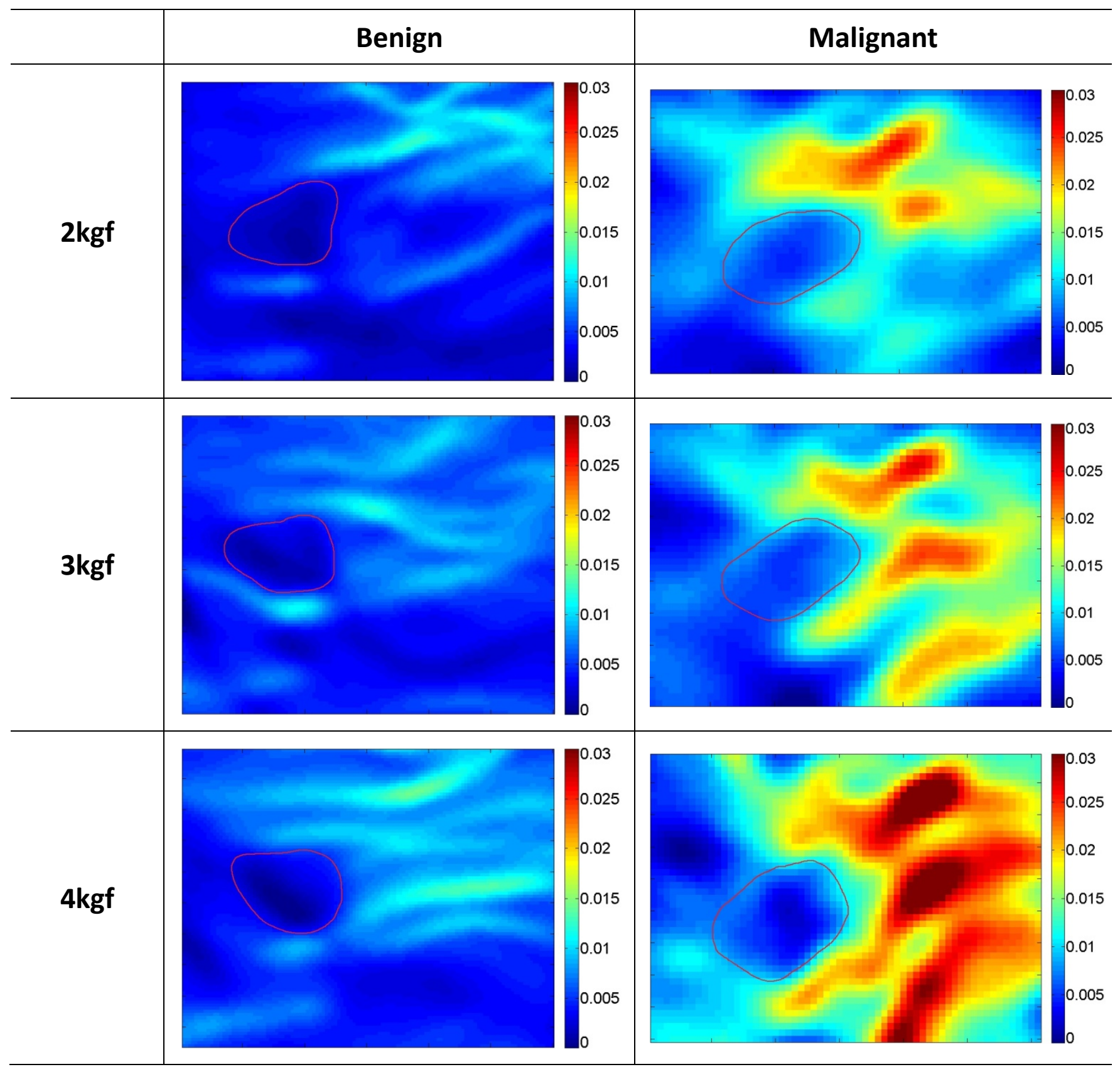

Figure 5.5 Breast 2D elastography imaging at three compression levels, where the colorbar represents strain difference. 
In order to emphasize the degree of tissue nonlinearity, strain differences between suspected mass and the healthy tissues, were computed over the three pre-compression levels using a selection technique very similar to the one presented in Fig. 4.6. The computations were based on slice by slice calculations, in which each slice containing the target mass was used to calculate strain values along with the surrounding soft tissue. Strain mean values along with standard deviations for the whole volume were plotted at each pre-compression level. Care was taken to select regions at the same imaging depth, so that both stiff and soft regions would be approximately subjected to the same stress level. The calculated strain difference values were calculated for the four volumes that were presented in the last section, and plotted in Fig's. 5.6 and 5.7 for benign and malignant cases, respectively. The figures present mass strain differences plots along with the associated mass relative stiffness for the four demonstrated cases.

Case \# 1
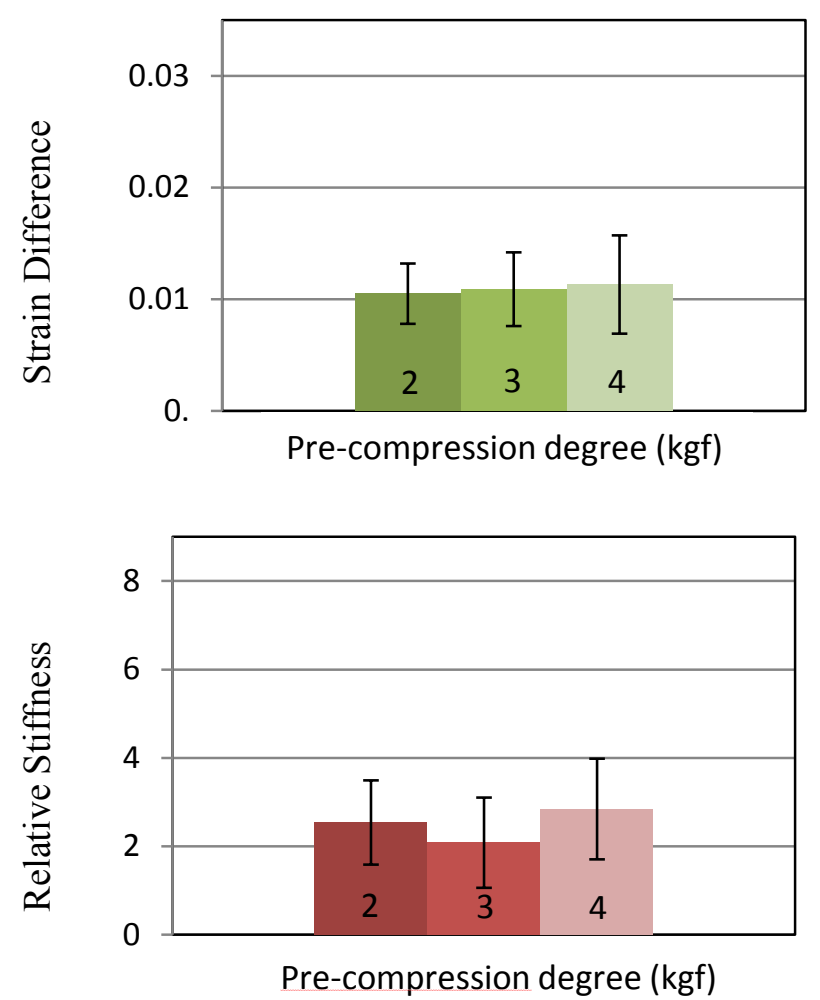

Case \# 5
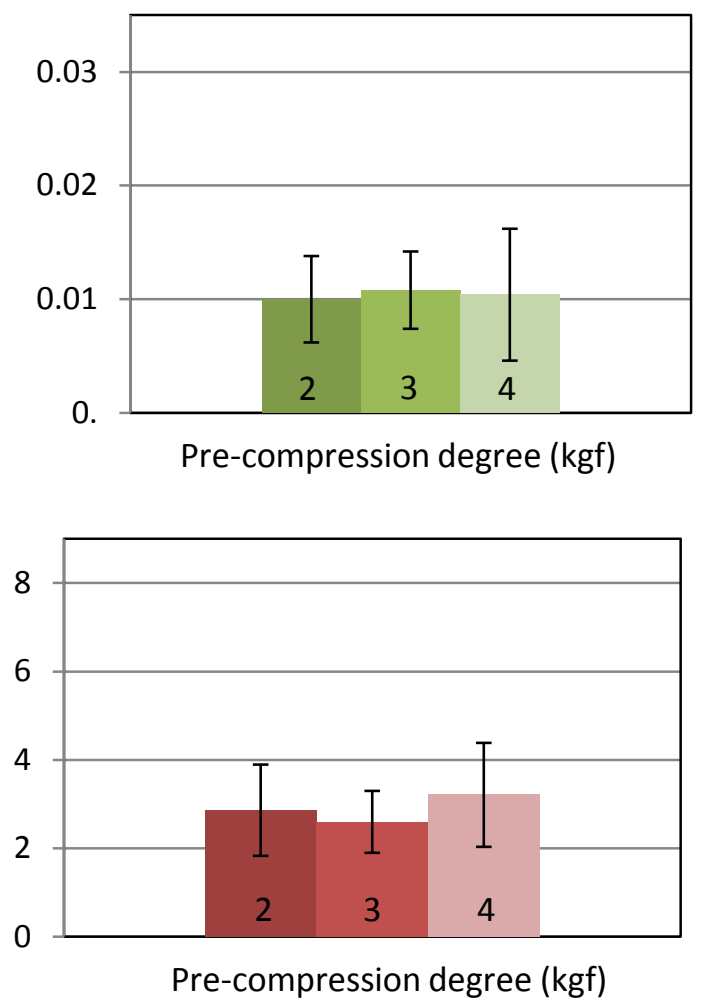

Figure 5.6 Benign breast masses behavior.

From the figures we notice that, the strain difference plots for case 1 and 5 showed an insignificant increase in difference values with increasing the pre-compression pressure. The 
relative stiffness followed nearly the same behavior. Referring back to Fig. 3.12; the biomechanical model of the breast tissue, we can conclude that cases 1 and 5 were benign masses. The observed insignificant increase in values indicated benign mass behavior that followed a force-strain relationship similar to the normal tissue, but with shifted strain values, as in Fig 3.12. Biopsy diagnosis revealed that the case number 1 had a history of bilateral fibroadenomas, and the mass type was benign fibroadenoma. In addition, case number 5 biopsy revealed a fibrocystic change that the formation of the mass. These confirmed our pre-diagnosis of the masses being benign.

Case \# 2
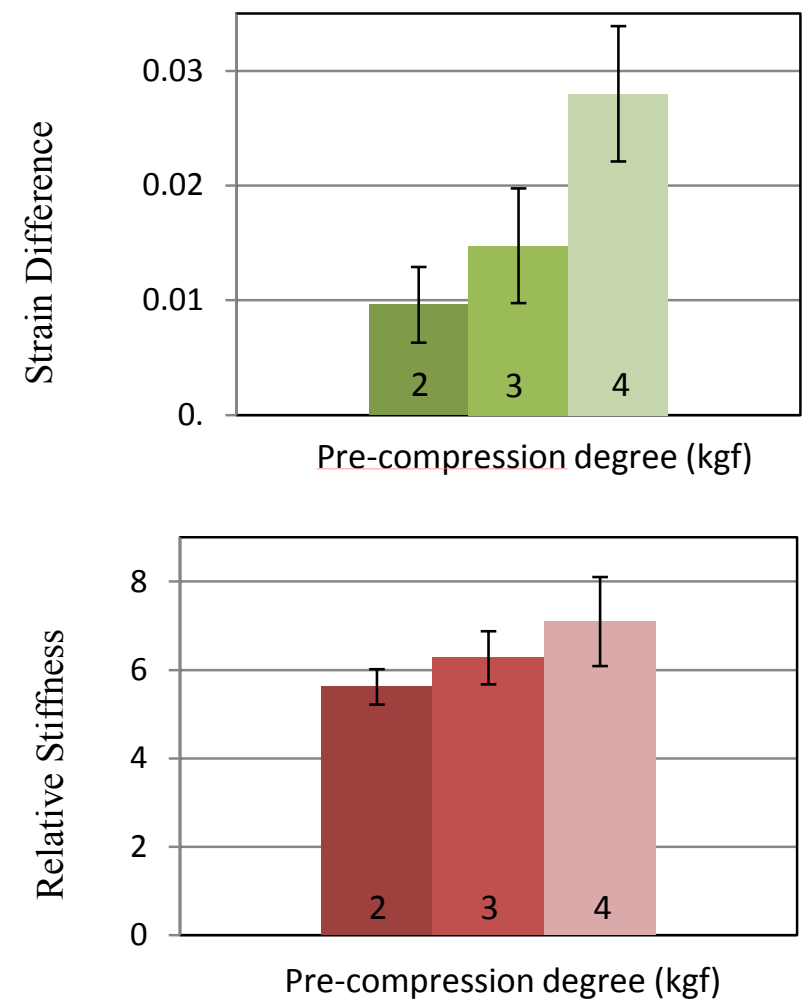

Case \# 8
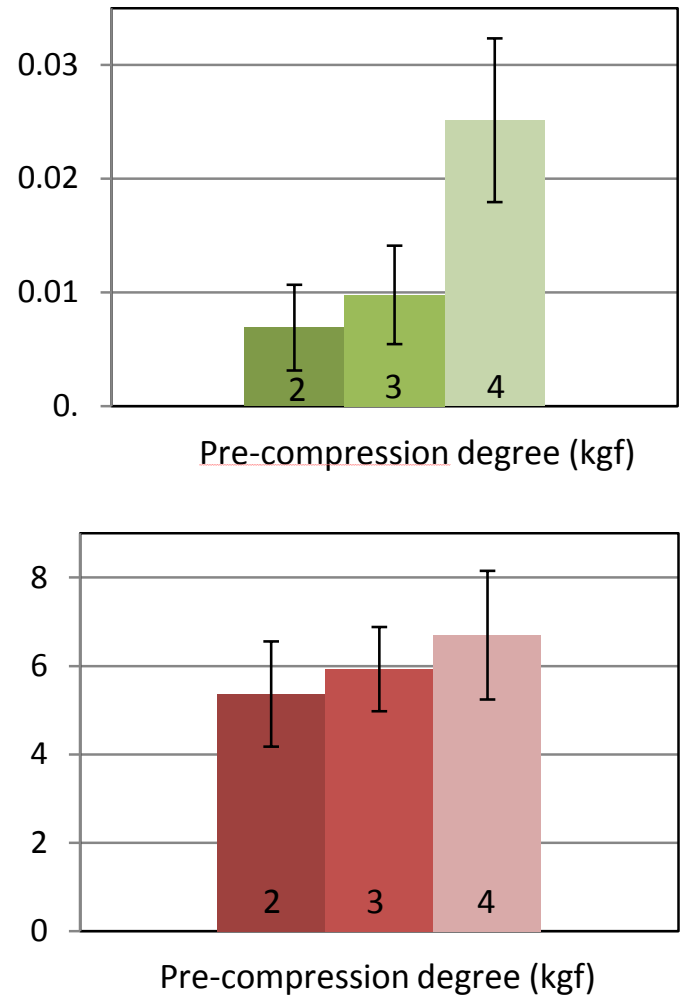

Figure 5.7 Malignant breast masses behavior.

However, cases 2 and 8 plots displayed a significant increase in strain difference values with increasing the pre-compression levels, especially between F4 and F3 levels where the values raised the most. The relative stiffness also followed nearly the same behavior, but with almost a gradual linear increase in values. The observed significant increase in values indicated a malignant mass behavior that followed a steep nonlinear force-strain relationship, as shown in Fig 3.12. Biopsy diagnosis revealed that the case number 2 had a malignant mass with invasive ductal carcinoma. 
Additionally, case number 8 biopsy also revealed a malignant mass with invasive ductal carcinoma having high grade ductal carcinoma in situ, which means an aggressive type of cancer. These confirmed our pre-diagnosis of the masses being malignant.

Please note that the ten examined breast masses have all shown the same nonlinear behavior as in Fig's. 5.6 and 5.7, according to the mass type in each case. The four presented cases are selected examples from the study subjects.

For the cases involved in the study, the overall average strain differences values were $0.011 \pm$ 0.005 and $0.018 \pm 0.014$ for benign and malignant masses at different compression levels, respectively. Although those values do not provide a clear classification boundary between mass types, individual case analysis of the strain difference curves versus the applied compression provided a very useful classifier. That classifier is the estimated nonlinear classification parameters.

\subsubsection{Quantifying degrees of nonlinearity}

Equations 3.12, 3.13 and 3.14 were used to fit the strain differences values using the powerlaw, linear and logarithmic relationships. Curve fitting was performed using least absolute residual method. Figure 5.8 shows curve fittings for examples of benign and malignant cases corresponding to patients 1 and 2, respectively. Generally, the benign curves exhibited a relatively high slope due to the relative steadiness of the strain difference values with the increasing pre-compression force. On the other hand, the slope of the malignant curves was relatively low, as the strain difference values were rapidly increasing with the elevating pre-compression level. This trend reflects the nonlinear biomechanical behavior of the malignant masses, wherein the more stress they experience, the higher stiffness they exhibit, and hence lower mass strains were observed. The lower strain with respect to the surrounding healthy tissue was captured using the strain differences measured at three different loads. These plots showed a good agreement with our proposed hypotheses in Fig. 3.12. This shows that the three fitting relationships are all good models for fitting the force-strain difference curves in vivo. The power fit was slightly sensitive to the benign values, where in few cases the power fit of the $(\Delta \varepsilon, \mathrm{f})$ benign data fails to reflect the true slope of the benign trend, while fitting the (f, $\Delta \varepsilon$ ) benign data was always successful and insensitive to the high benign trends. It is worth mentioning that the fitted equations do not directly model the biomechanical properties of the tissue under investigation, but rather measure the degree of tissue nonlinearity, which helps indicate the mass's type. 
Power Fit

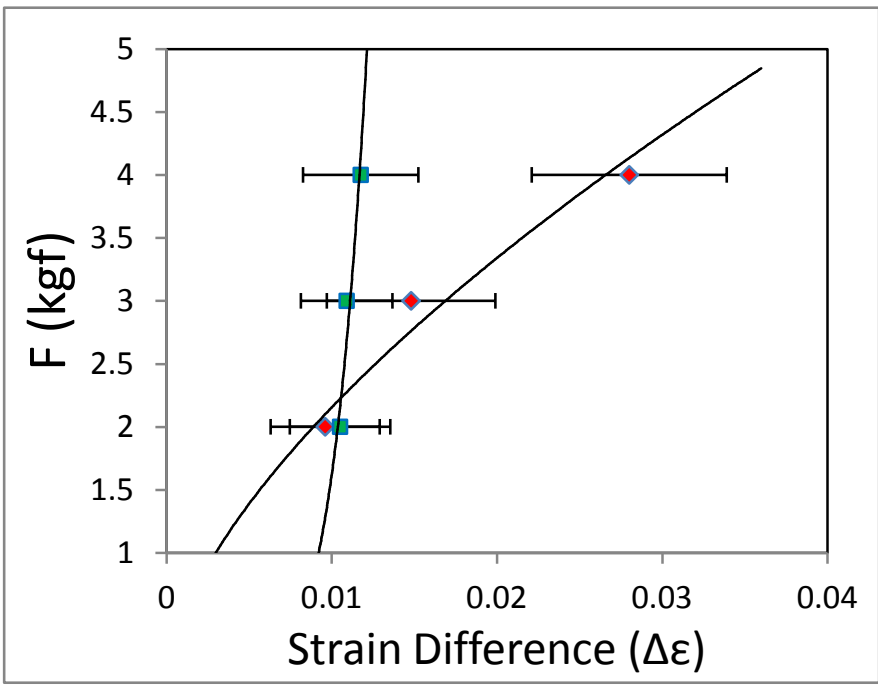

Linear Fit
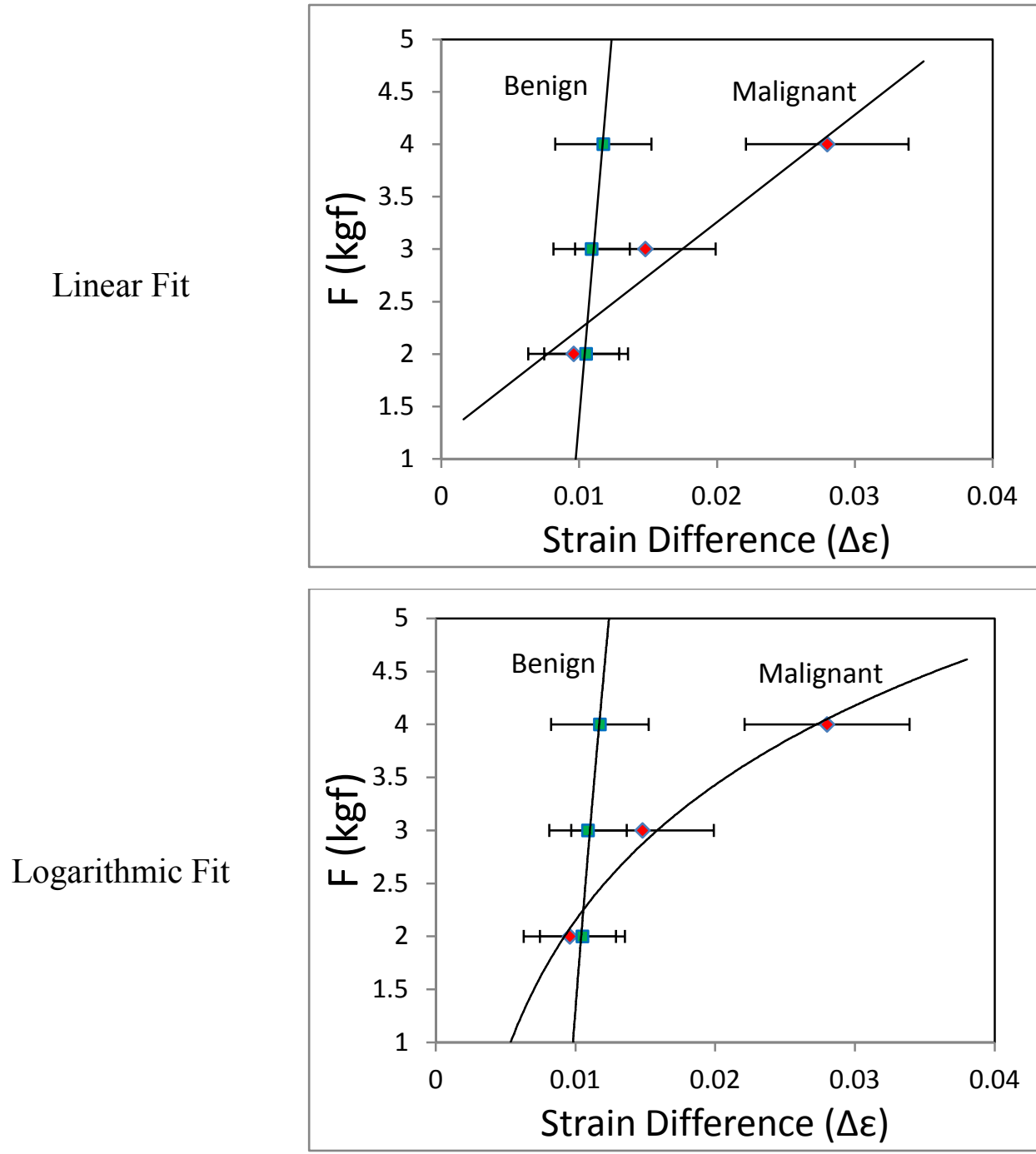

Figure 5.8 Examples of nonlinear parameters estimation using curve fitting. 
Nonlinear power parameters were accordingly calculated for each mass of the volunteered cases, and tabulated in Table 5.1. The overall average parameter values along with the corresponding standard deviations for the studied benign and malignant masses are listed in Table 5.2. A significant differentiation between the two mass types using the parameters is obvious in the tables. These results suggest that using the new nonlinear parameters would be very helpful in differentiating between benign and malignant masses noninvasively using elastography. In addition, the assumption that breast masses follow a nonlinear biomechanical behavior was justified.

Table 5.1 Nonlinear parameters estimated for all masses.

\begin{tabular}{c|cccccc|c}
\hline Patient & \multicolumn{2}{|c}{ Power Fit } & \multicolumn{2}{c}{ Linear Fit } & \multicolumn{2}{c}{ Logarithmic Fit } & \multirow{2}{*}{ Diagnosis } \\
Number & $\boldsymbol{L o g}(\boldsymbol{m})$ & $\boldsymbol{n}$ & $\boldsymbol{a}$ & $\boldsymbol{b}$ & $\boldsymbol{c}$ & $\boldsymbol{d}$ & \\
\hline $\mathbf{1 - A}$ & 18.54 & 0.11 & 2438.54 & -23.6 & 26.59 & 123.18 & Benign \\
\hline $\mathbf{1 - B}$ & 9.64 & 0.23 & 1643.80 & -10.5 & 13.68 & 68.67 & Benign \\
\hline $\mathbf{5}$ & 10.54 & 0.23 & 2248.17 & -8.56 & 7.12 & 63.96 & Benign \\
\hline $\mathbf{6}$ & 5.15 & 0.45 & 941.12 & -4.17 & 10.57 & 37.77 & Benign \\
\hline $\mathbf{7}$ & 6.85 & 0.17 & 568.16 & -7.69 & 11.56 & 45.03 & Benign \\
\hline $\mathbf{2}$ & 1.59 & 1.58 & 102.25 & 1.21 & 1.85 & 10.65 & Malignant \\
\hline $\mathbf{4}$ & 1.61 & 1.79 & 159.96 & 1.47 & 1.64 & 10.83 & Malignant \\
\hline $\mathbf{8}$ & 1.39 & 2.05 & 94.84 & 1.68 & 1.44 & 9.39 & Malignant \\
\hline $\mathbf{9}$ & 1.94 & 1.31 & 172.29 & 0.89 & 2.24 & 13.03 & Malignant \\
\hline $\mathbf{1 0}$ & 1.74 & 1.81 & 270.96 & 1.49 & 1.64 & 11.72 & Malignant \\
\hline
\end{tabular}

Table 5.2 Mean and standard deviation values of the mass's classification parameters.

\begin{tabular}{c|ccccc}
\hline \multirow{2}{*}{ Power Fit } & Parameter & \multicolumn{2}{c}{ Benign } & \multicolumn{2}{c}{ Malignant } \\
& $\begin{array}{c}\text { Log Scale, } \\
\text { Log(m) }\end{array}$ & 9.637 & 4.626 & 1.613 & 0.179 \\
\cline { 2 - 6 } & Exponent, $\boldsymbol{n}$ & 0.238 & 0.129 & 1.709 & 0.277 \\
\hline \multirow{2}{*}{ Linear Fit } & Slope, $\boldsymbol{a}$ & 1643.8 & 724.38 & 159.96 & 63.317 \\
\cline { 2 - 6 } & Intersection, $\boldsymbol{b}$ & -8.563 & 6.738 & 1.349 & 0.273 \\
\hline \multirow{3}{*}{ Logarithmic Fit } & Scaling, $\boldsymbol{c}$ & 13.91 & 7.48 & 1.76 & 0.30 \\
\cline { 2 - 6 } & Shift, $\boldsymbol{d}$ & 67.72 & 33.55 & 11.13 & 1.35 \\
\hline
\end{tabular}




\subsubsection{Classification Methods Comparison}

The three traditional mass classification features usually used in the literature are:

1. Appearance of the stiff mass in elastograms.

2. Relative stiffness value (RS).

3. Relative volume (RV) of the mass between B-mode images and elastograms.

The first feature was demonstrated earlier in the 3D imaging section of this chapter, and it was found that 3D elastography improves the interpretability of the elastograms with the clear view of stiff masses inside the soft tissue.

The second classification feature or parameter is the mass relative stiffness. Figure 5.5 demonstrated calculations of the target masses over the three pre-compression levels. For comparison purposes the average value over the three compression levels was calculated for each case involved in the study, as Table 5.3 shows. The calculations resulted in relative stiffness average values of $2.348 \pm 0.776$ and $4.710 \pm 1.690$ for the benign and malignant masses, respectively. Those average values agrees well with the previously reported values of benign and malignant masses in the literature, in which a threshold value of 4.8 was considered [37]. Utilizing the relative stiffness ratios only as a classification parameter suffers from an overlap in the values depicted by benign and malignant masses [81-83], and in some cases it is hard to diagnose the mass based on this parameter. One case had an average relative stiffness of 2.06 , and it was mistakenly classified as benign using this parameter, as the biopsy showed a malignant mass. Therefore, this parameter was computed mainly through the study to quantitatively verify our methods with the literature, but not for the purpose of mass classification. Multi-compression relative stiffness values can also be used to estimate the nonlinear parametric classification. However, strain differences provided better emphasis on the nonlinearity of stiff masses and made a larger separation margin between the parameter values for malignant and benign masses.

The third parameter; relative volume, required the calculation of two volumes for each mass, using 3D elastography and B-mode volumes as well. Breast mass volume calculations were performed as the summation of mass's areas in the slices constituting the elastographic/B-mode volumes, as described in the methods chapter. The relative volume calculations, which are the ratios between the mass volume in the 3D axial strain and the 3D B-mode (Ax/B), are shown in Table 5.4. 
Table 5.3 Mass classification using relative stiffness (RS).

\begin{tabular}{c|cccccccccc}
\hline $\begin{array}{c}\text { Patient } \\
\text { Number }\end{array}$ & $\mathbf{1 - 1}$ & $\mathbf{1 - 2}$ & $\mathbf{5}$ & $\mathbf{6}$ & $\mathbf{7}$ & $\mathbf{2}$ & $\mathbf{4}$ & $\mathbf{8}$ & $\mathbf{9}$ & $\mathbf{1 0}$ \\
\hline RS & 3.20 & 2.50 & 2.90 & 1.84 & 1.31 & 6.30 & 2.06 & 6.0 & 4.29 & 4.90 \\
\hline Diagnosis & Benign & Benign & Benign & Benign & Benign & Malignant & Malignant & Malignant Malignant Malignant \\
\hline
\end{tabular}

Table 5.4 Mass classification using relative volume parameter.

\begin{tabular}{c|cccccccccc}
\hline $\begin{array}{c}\text { Mass } \\
\text { Number }\end{array}$ & $\mathbf{1 - 1}$ & $\mathbf{1 - 2}$ & $\mathbf{5}$ & $\mathbf{6}$ & $\mathbf{7}$ & $\mathbf{2}$ & $\mathbf{4}$ & $\mathbf{8}$ & $\mathbf{9}$ & $\mathbf{1 0}$ \\
\hline B-Mode & 196.52 & 332.91 & 37.9 & 264.6 & 16.1 & 146.5 & 44.2 & 247.7 & 321.0 & 375.37 \\
\hline $\begin{array}{c}\text { Axial } \\
\text { Strain }\end{array}$ & 210.81 & 322.16 & 17.3 & 239.5 & 15.4 & 242.9 & 119.4 & 812.8 & 656.86 & 589.11 \\
\hline $\begin{array}{c}\text { Ratio } \\
\text { (Ax/B) }\end{array}$ & 1.07 & 0.97 & 0.45 & 0.91 & 0.96 & 1.66 & 2.7 & 3.28 & 2.05 & 1.57 \\
\hline $\begin{array}{c}\text { Diagnosis } \\
\text { Benign }\end{array}$ & Benign & Benign & Benign & Benign & Malignant & Malignant & Malignant & Malignant Malignant \\
\hline
\end{tabular}

Mass diagnosis was verified with the biopsy pathological results. Differentiation between mass types using this parameter is apparent in the table, in which malignant cases exhibited values of more than one, and benign cases had values almost equal to one. The overall average relative mass volume values were $0.872 \pm 0.243$ and $2.245 \pm 0.736$ for benign and malignant masses, respectively. The performance of relative mass size parameter between elastography and B-mode was generally good in the classification process in this study. However, a difficulty exists using this parameter as it requires the mass to be quite clear in the B-mode images, which is not the case for many kinds of breast masses, where the mass boundary is vague. Hence many clinical studies suggested special care during performing such measurements, as it may cause erroneous decisions $[37,84,114]$.

The accumulated nonlinear parameters values for all masses are shown in Fig. 5.9 a-f) using box plots, to emphasize the discrimination regions between the benign and malignant mass types. 


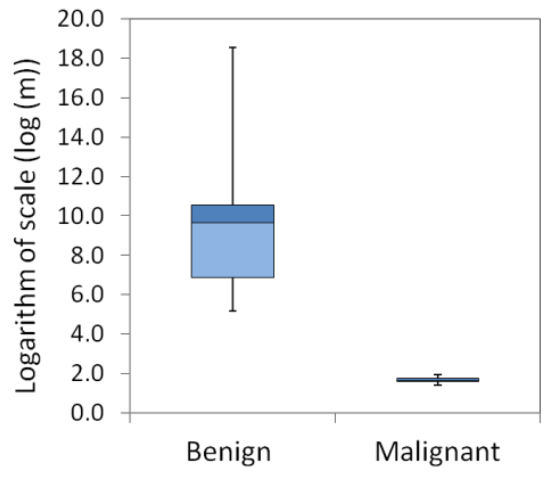

a)

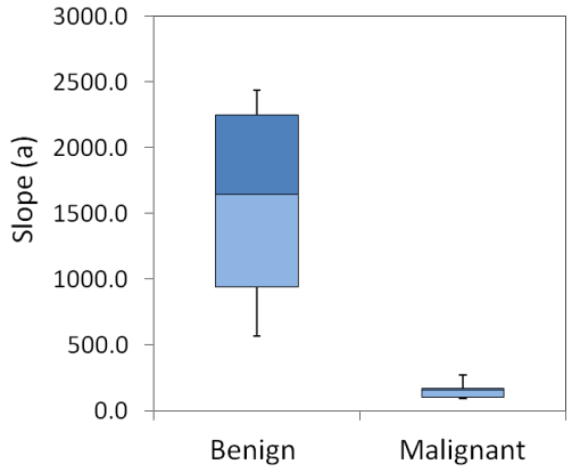

c)

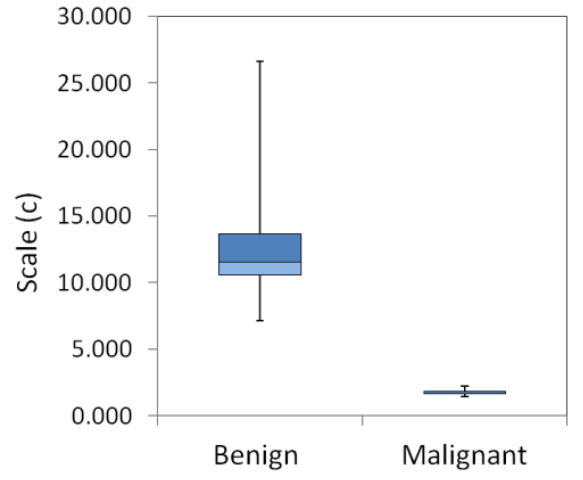

e)

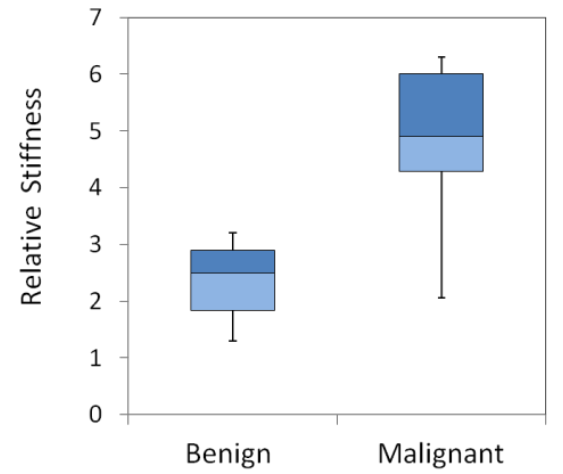

g)

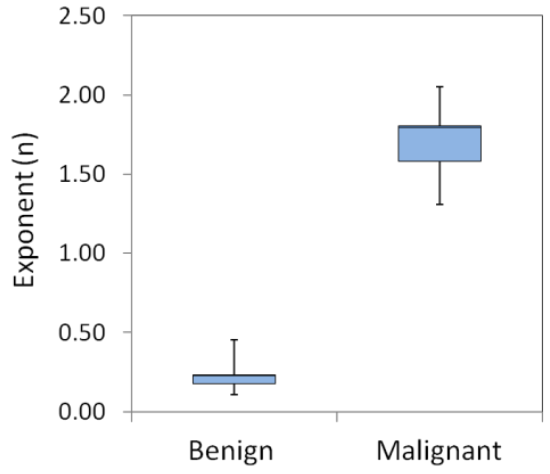

b)

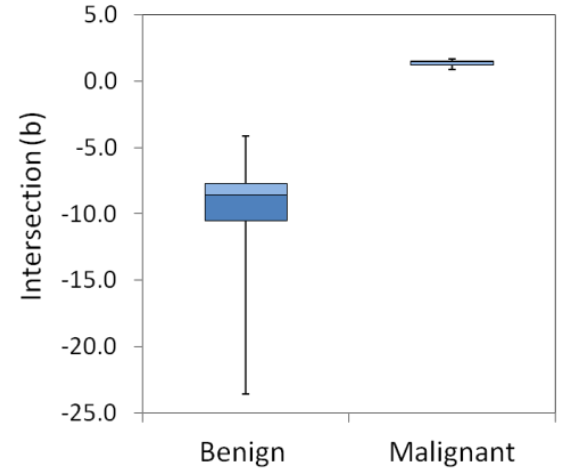

d)

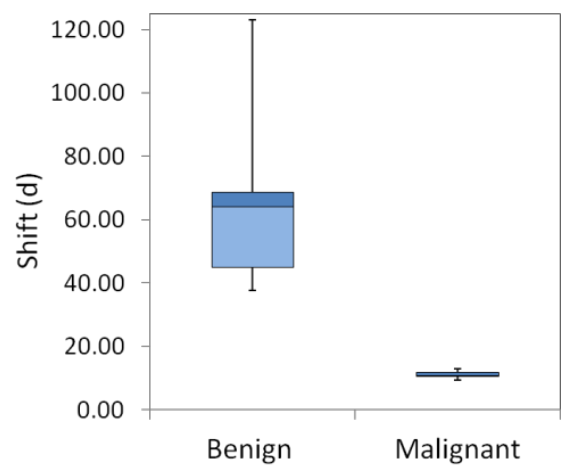

f)

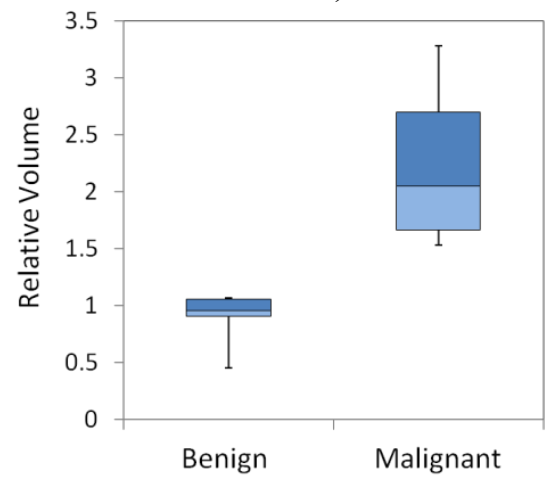

h)

Figure 5.9 Classification parameters performance comparison: a-f) Nonlinear classification parameters estimated using: a-b) power-law, c-d) linear, and e-f) logarithmic relationships. g-h) Conventional elastography mass classification using: g) Relative stiffness. h) Relative volume. 
Box plots were employed to graph the classification parameters, in which the error bars represent the minimum and maximum values, the red line represents the median parameter value, and the lower and upper edges of the box represent 25 and 75 percentiles of the values, respectively [115]. The accumulated relative stiffness and relative mass volumes for each mass type category are also shown in Fig. 5.9 g-h), respectively. The power exponent nonlinear parameters $n$ outperforms the rest of the parameters, as shown in Fig. 5.9 b), as it exhibits the most confined and separated regions for each mass type. Also the parameter $n$ has a larger discrimination region than the other parameters with a lower overlap between the two mass classes. Additionally, statistical t-tests elucidated that the classification parameters were statistically different between the benign and malignant tumors. The calculated p-values for each parameter are listed in Table 5.5. Please note, that the scaling factor $m$ of the power fit was not statistically significant by itself, as its p-value was 0.374. However the logarithm of the scaling factor was found to be statistically significant with a $p$ value of 0.0213 . The statistical tests revealed that all the parameters were significant within $5 \%$ significance level, except the exponent $n$ that was statistically highly significant at a level of $0.1 \%$. Using the power exponent $n$, an initial threshold of 1 was suggested and used through the study to classify breast masses, but this value can be refined with further studies that involve a larger number of human test subjects.

Based on this study, a general comparison between the three elastographic classification methods is demonstrated in Table 5.6. These advantages would make the classification process more sensitive, specific and less confusing for the clinicians.

Table 5.5 Statistical significance of the mass classification parameters.

\begin{tabular}{c|cccccccc}
\hline Parameter & $\log (\mathbf{m})$ & $\mathbf{n}$ & $\mathbf{a}$ & $\mathbf{b}$ & $\mathbf{c}$ & $\mathbf{d}$ & $\mathbf{R S}$ & $\mathbf{R V}$ \\
\hline p-value & 0.0213 & 0.000562 & 0.0174 & 0.0212 & 0.0221 & 0.0195 & 0.0318 & 0.0232 \\
\hline
\end{tabular}


Table 5.6 Mass classification methods comparison.

\begin{tabular}{|c|c|c|}
\hline $\begin{array}{l}\text { Classification } \\
\text { Method }\end{array}$ & Advantages & Disadvantages \\
\hline $\begin{array}{c}\text { Proposed } \\
\text { Method } \\
\text { (Tissue } \\
\text { nonlinearity) }\end{array}$ & $\begin{array}{l}\text { - Accurate mass differentiation [113]. } \\
\text { - Addresses tissue biomechanics and } \\
\text { nonlinearity. } \\
\text { - Can be extended to classify other } \\
\text { organ's tumors }[18,116] \text {. }\end{array}$ & $\begin{array}{l}\text { - Requires multi-compression. } \\
\text { - Requires force monitoring. } \\
\text { - Requires operator training. }\end{array}$ \\
\hline Relative volume & - Good mass differentiation [32]. & $\begin{array}{l}\text { - Suffers from operator subjectivity } \\
\text { [ } 84,117] \text {. } \\
\text { - Some malignant masses are not } \\
\text { well bounded on B-mode images, } \\
\text { lead to inaccurate results [37]. } \\
\text { - Limited for breast masses [116]. }\end{array}$ \\
\hline Relative stiffness & $\begin{array}{l}\text { - Can be used to classify other } \\
\text { organ's tumors }[18,116] \text {. }\end{array}$ & $\begin{array}{l}\text { - Suffers from operator subjectivity } \\
{[16,84] \text {. }} \\
\text { - Parameter overlaps between mass } \\
\text { types }[37,116] .\end{array}$ \\
\hline
\end{tabular}

\subsubsection{Repeatability study}

The results presented in the previous subsection were based on a single elastographic data acquisition for each mass. During the clinical study, double data acquisitions were obtained, to ensure that a useful data were present for each case by the end of the examination. This second volumetric data set was used to conduct a repeatability study to evaluate the estimation variation of the nonlinear classification parameter and the mass volume, on the same human subject. Analysis of the second data set was possible for the breast masses that were involved in the study, except for two masses. Mass number 1 of the first patient was not visible in the produced elastograms because of the change in the viewing field for the second data set. Mass number 6 did not have second readings either, where the second data set was not attainable for this patient.

Following the same procedure as for the first data set, new measurements were performed for eight masses to estimate the nonlinear classification parameter $n$. The two readings per mass were averaged and the corresponding standard deviation was calculated, as listed in Table 5.6. The masses that do not have a second reading were given a standard deviation of zero, as they have only 
one measurement. It can be noticed from the table that the new readings are in the same range of the first ones, wherein the classification parameter did not fail to classify the mass type in the second round of measurements. This was also obvious in Fig 5.10 that represents a graphical plot of Table 5.7. Based on the new combined measurements, the plot portrays a clear discrimination region between the benign and malignant masses, in which benign values were less than 0.5 , and the malignant values were higher than 1 . This benign/malignant discrimination region was shaded in the plot in Fig. 5.10.

Table 5.7 Repeatability of the nonlinear parameter using double volumetric acquisitions for the same patients.

\begin{tabular}{c|cc}
\hline Mass Number & Nonlinear Parameter $\boldsymbol{n}$ & STD \\
\hline $\mathbf{1 - A}$ & 0.11 & 0 \\
\hline $\mathbf{1 - B}$ & 0.266526 & 0.039274 \\
\hline $\mathbf{2}$ & 1.734139 & 0.153861 \\
\hline $\mathbf{4}$ & 1.656037 & 0.141037 \\
\hline $\mathbf{5}$ & 0.310079 & 0.083021 \\
\hline $\mathbf{6}$ & 0.452284 & 0 \\
\hline $\mathbf{7}$ & 0.235519 & 0.060081 \\
\hline $\mathbf{8}$ & 1.73709 & 0.31209 \\
\hline $\mathbf{9}$ & 1.180216 & 0.130916 \\
\hline $\mathbf{1 0}$ & 1.927942 & 0.121258 \\
\hline
\end{tabular}

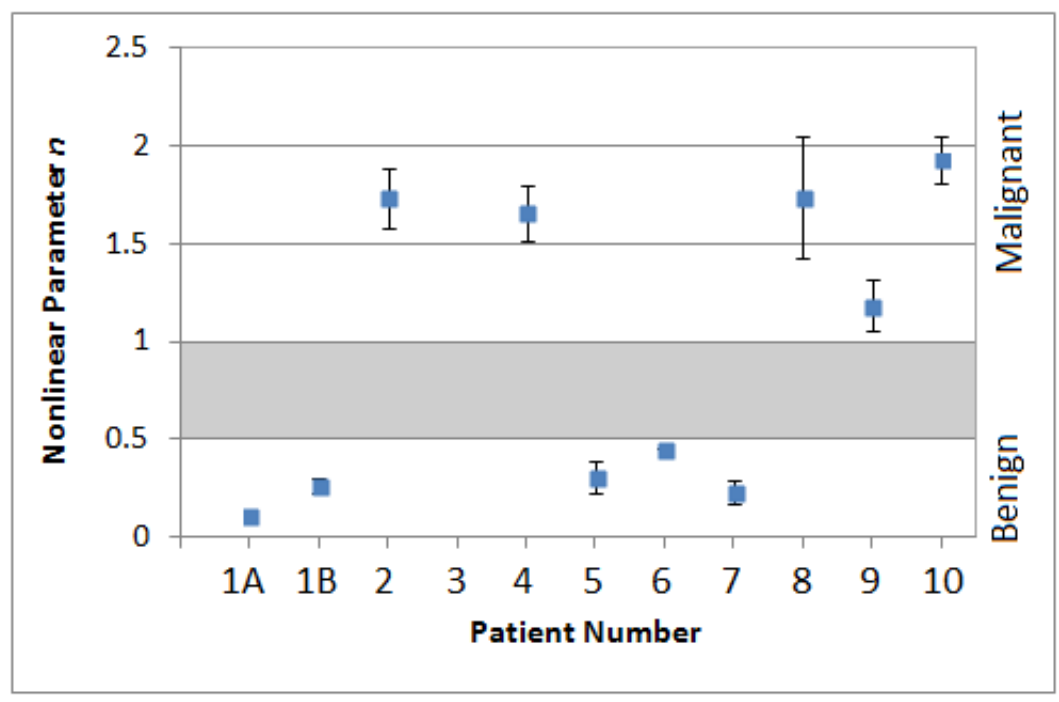

Figure 5.10 A plot showing the discrimination margin between benign and malignant masses using repeatable estimations of the nonlinear classification parameter $n$. 
Another measure of the study repeatability was to calculate mass volumes for the second data set. The two volume estimations per mass were averaged and the corresponding standard deviation was calculated and listed in Table 5.8. It can be concluded from the table that the new estimations are within the same range of the first ones, except for mass number 8 . The reason behind this high volume variation was the different breast imaging field of view in the two acquired data sets, which resulted in dissimilar mass elastographic volumes and hence different volume calculations.

Table 5.8 Repeatability of the mass's volume calculation $\left(\mathrm{mm}^{3}\right)$ using double volumetric acquisitions.

\begin{tabular}{c|cc}
\hline Mass Number & Average Volume & STD \\
\hline 1-A & 227.42 & 23.48 \\
\hline $\mathbf{1 - B}$ & 322.16 & 0 \\
\hline $\mathbf{2}$ & 245.57 & 3.78 \\
\hline $\mathbf{4}$ & 121.95 & 3.60 \\
\hline $\mathbf{5}$ & 20.10 & 3.96 \\
\hline $\mathbf{6}$ & 239.50 & 0 \\
\hline $\mathbf{7}$ & 15.88 & 0.68 \\
\hline $\mathbf{8}$ & 742.95 & 98.78 \\
\hline $\mathbf{9}$ & 642.80 & 19.89 \\
\hline $\mathbf{1 0}$ & 586.38 & 3.877 \\
\hline
\end{tabular}

An additional form of repeatability judgment is to calculate the classification parameter based on multiple 2D measurements. This method is different from the previously described 3D method, which estimates the parameter based on the overall force-strain difference values obtained from the whole volume portraying the mass. The 2D measurement method uses individual slices instead of volumes, which allows for investigating the values repeatability within individual volumes, as well as between similar mass types. Table 5.9 lists five parameter readings per mass, with the corresponding mean value, standard deviation within, and standard deviation between the other similar cases. Only mass number 7 had four readings, as that mass was very small and could only be seen in four slices. These results show that 2D based calculations also succeeded to discriminate between benignly and malignancy, with acceptable variations that had an average value of $20 \%$. The standard deviation between similar mass types was also comparable, indicating the close parameter values between the corresponding mass diagnoses. 
Table 5.9 Calculation of the nonlinear parameter $n$ based on multiple 2D readings.

\begin{tabular}{|c|c|c|c|c|c|c|c|}
\hline Diagnosis & Mass Type & Mass \# & Reading & $\mathbf{n}$ & Mean n & SD Within & SD Between \\
\hline \multirow{5}{*}{ Benign } & \multirow{2}{*}{ Fibroadenoma } & $1 \mathrm{~A}$ & $\begin{array}{l}1 \\
2 \\
3 \\
4 \\
5\end{array}$ & $\begin{array}{l}0.119 \\
0.189 \\
0.256 \\
0.172 \\
0.120 \\
\end{array}$ & 0.171 & 0.057 & \multirow{2}{*}{0.077} \\
\hline & & $1 \mathrm{~B}$ & $\begin{array}{l}1 \\
2 \\
3 \\
4 \\
5\end{array}$ & $\begin{array}{l}0.387 \\
0.303 \\
0.231 \\
0.313 \\
0.166\end{array}$ & 0.280 & 0.084 & \\
\hline & \multirow{2}{*}{ Fibrocystic Changes } & 5 & $\begin{array}{l}1 \\
2 \\
3 \\
4 \\
5\end{array}$ & $\begin{array}{l}0.286 \\
0.160 \\
0.409 \\
0.321 \\
0.295\end{array}$ & 0.294 & 0.089 & \multirow[t]{2}{*}{0.042} \\
\hline & & 7 & $\begin{array}{l}1 \\
2 \\
3 \\
4 \\
\end{array}$ & $\begin{array}{l}0.293 \\
0.211 \\
0.285 \\
0.152 \\
\end{array}$ & 0.235 & 0.067 & \\
\hline & Fibroadipose & 6 & $\begin{array}{l}1 \\
2 \\
3 \\
4 \\
5 \\
\end{array}$ & $\begin{array}{l}0.330 \\
0.293 \\
0.420 \\
0.176 \\
0.453 \\
\end{array}$ & 0.334 & 0.110 & 0.000 \\
\hline \multirow{5}{*}{ Malignant } & \multirow{4}{*}{ IDC } & 2 & $\begin{array}{l}1 \\
2 \\
3 \\
4 \\
5\end{array}$ & $\begin{array}{l}1.692 \\
1.509 \\
1.564 \\
1.355 \\
1.595 \\
\end{array}$ & 1.543 & 0.124 & \multirow{4}{*}{0.467} \\
\hline & & 8 & $\begin{array}{l}1 \\
2 \\
3 \\
4 \\
5\end{array}$ & $\begin{array}{l}2.219 \\
1.942 \\
2.185 \\
2.301 \\
1.782\end{array}$ & 2.086 & 0.216 & \\
\hline & & 9 & $\begin{array}{l}1 \\
2 \\
3 \\
4 \\
5\end{array}$ & $\begin{array}{l}1.153 \\
1.432 \\
1.402 \\
0.965 \\
1.070 \\
\end{array}$ & 1.204 & 0.205 & \\
\hline & & 10 & $\begin{array}{l}1 \\
2 \\
3 \\
4 \\
5\end{array}$ & $\begin{array}{l}2.140 \\
2.112 \\
2.341 \\
2.304 \\
2.093\end{array}$ & 2.198 & 0.115 & \\
\hline & ILC & 4 & $\begin{array}{l}1 \\
2 \\
3 \\
4 \\
5\end{array}$ & $\begin{array}{l}1.675 \\
1.971 \\
2.317 \\
2.018 \\
1.702\end{array}$ & 1.937 & 0.263 & 0.000 \\
\hline
\end{tabular}




\subsection{Derived Strains Imaging}

Equations 3.5 were calculated to evaluate the performance of the derived volumetric strain elastograms in vivo, for both benign and malignant masses. Figure 5.11 demonstrates the four elastographic volumes showing a cross sectional view of one of the benign masses. Normalization was performed for a fair comparison as described earlier. It is clear from the figure the enhancement in the mass's boundary, and the better distinction from the background soft tissue compared to the axial strain 3D elastogram. The maximum shear volumetric strain presented the best boundary enhancement then Von Mises strain and first principal strain, respectively. This agreed with the in vitro breast phantom results.

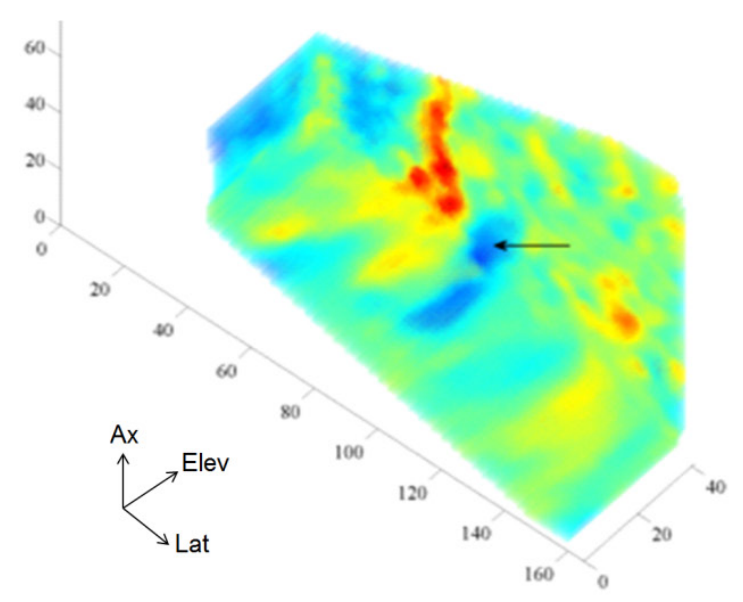

a)

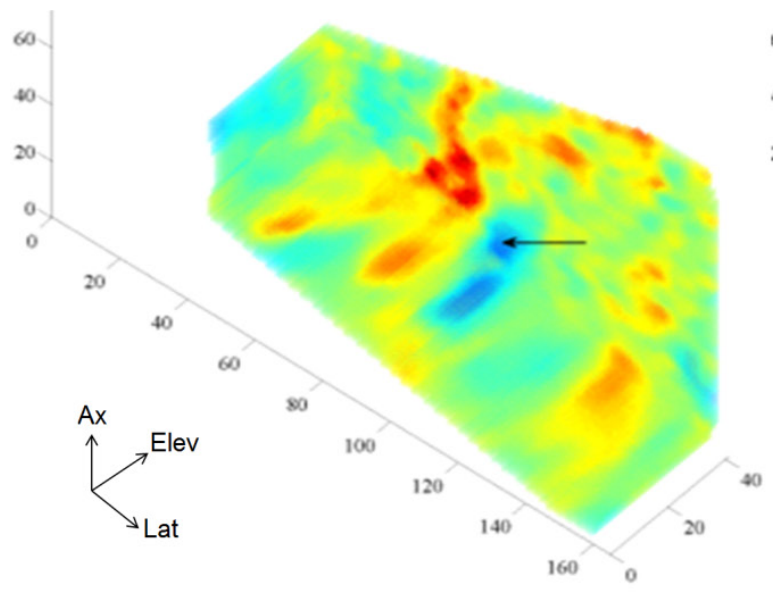

c)

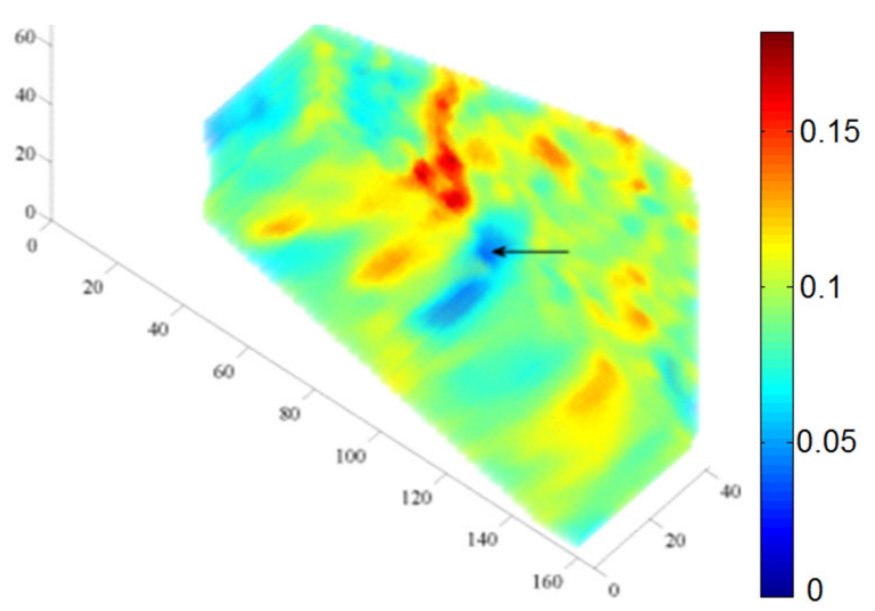

b)

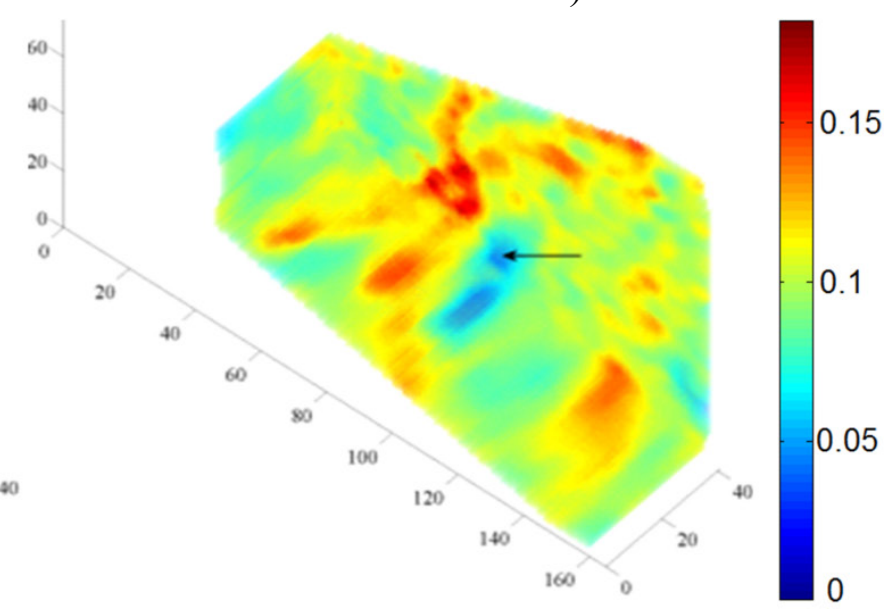

d)

Figure 5.11 In vivo volumetric strain elastograms for a benign case: a) Normal axial strain. b)

First principal strain. c) Von Mises strain. d) Maximum shear strain. The colorbar represents normalized strain. Axes are in pixel units, where 10 pixels equal $4 \mathrm{~mm}$. 
Figure 5.12 illustrates the four elastographic volumes showing a cross sectional view of a malignant mass. Once more, it was obvious from the figure the degree of distinction of the mass from the background soft tissue, by enhancing the mass's boundary. As shown in the breast phantom and the benign mass's cases, the maximum shear volumetric strain presents the best boundary enhancement then Von Mises strain and first principal strain, respectively. This observation showed that the derived strains can be utilized for imaging of benign and malignant masses as well.

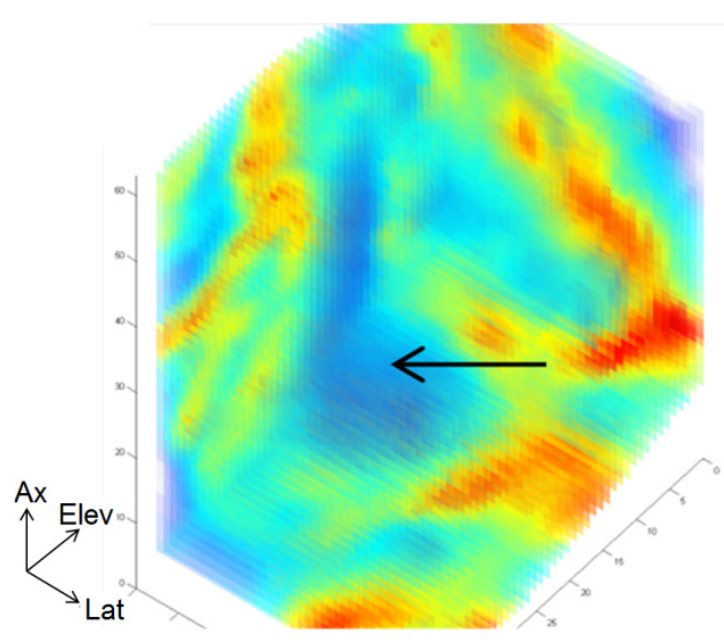

a)

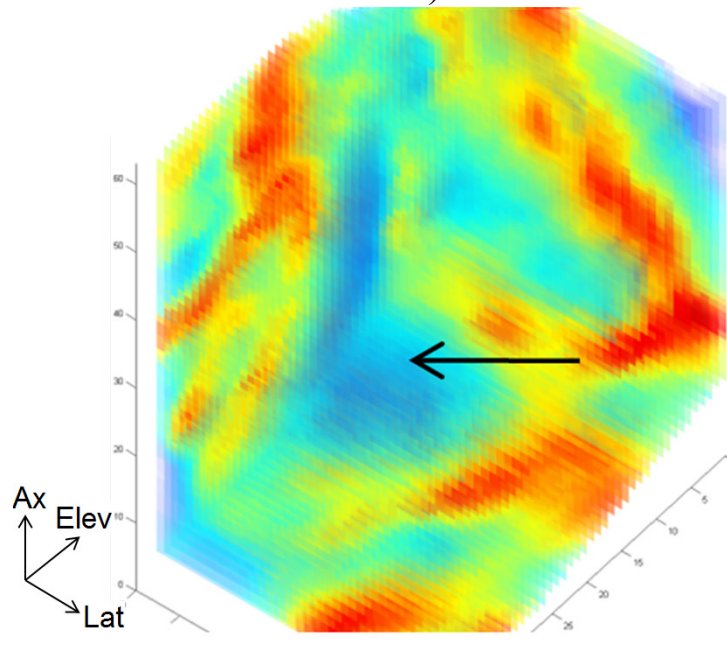

c)

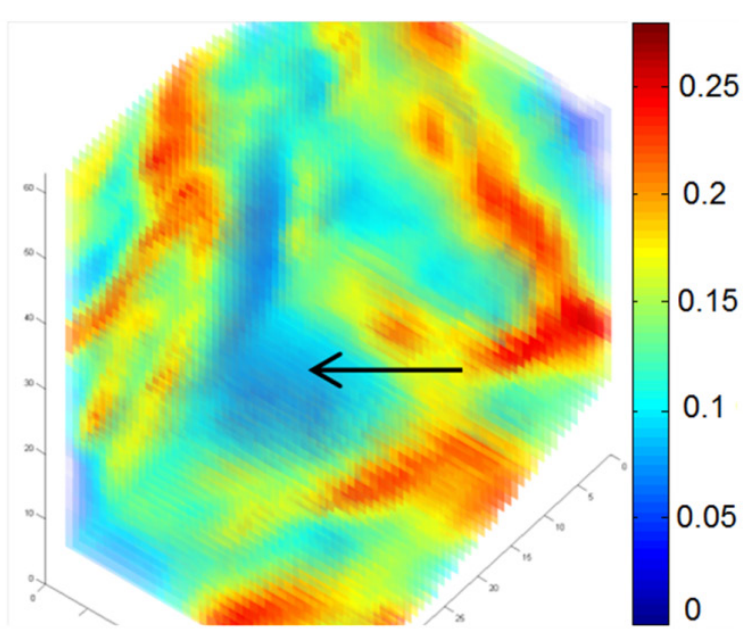

b)

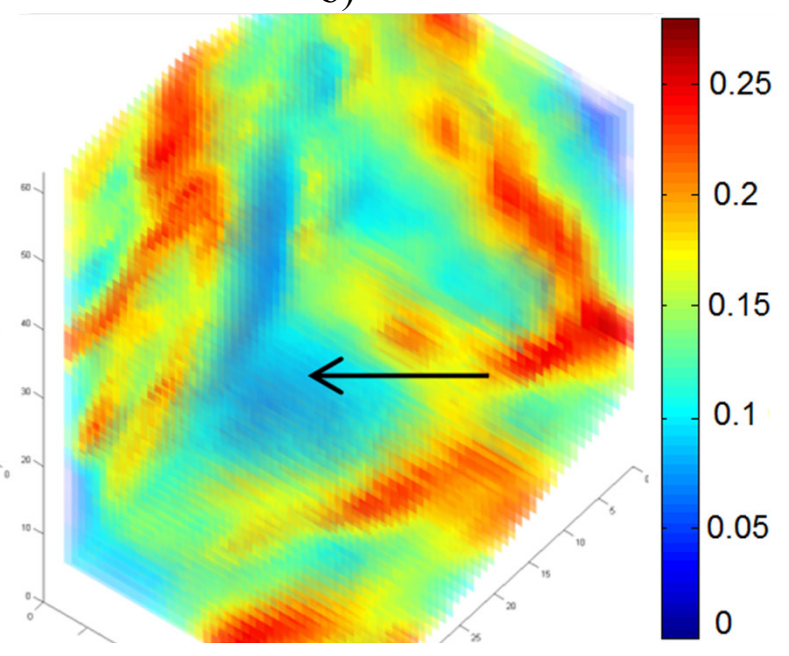

d)

Figure 5.12 In vivo volumetric strain elastograms for a malignant case: a) Normal axial strain. b) First principal strain. c) Von Mises strain. d) Maximum shear strain. The colorbar represents normalized strain. Axes are in pixel units, where 10 pixels equal $5 \mathrm{~mm}$. 
Furthermore, the derived volumetric strains were used to compute mass volume calculations for each case involved in the study. Volume calculation was performed on a slice by slice basis throughout the volumes as described before. The computed mass volume results are listed in Table 5.10. The results show that the calculated mass volumes were quite similar in the four derived volumetric strains, and therefore both shape and volume were almost preserved through the process.

Table 5.10 Breast mass volume $\left(\mathrm{mm}^{3}\right)$ calculations for all cases using manual segmentation.

\begin{tabular}{c|ccccccccccc}
\hline $\begin{array}{c}\text { Mass } \\
\text { Number }\end{array}$ & $\mathbf{1 - 1}$ & $\mathbf{1 - 2}$ & $\mathbf{2}$ & $\mathbf{4}$ & $\mathbf{5}$ & $\mathbf{6}$ & $\mathbf{7}$ & $\mathbf{8}$ & $\mathbf{9}$ & $\mathbf{1 0}$ \\
\hline Axial Strain & 210.81 & 322.16 & 242.9 & 119.4 & 17.3 & 239.5 & 15.4 & 812.8 & 656.86 & 589.11 \\
\hline $\begin{array}{c}\text { First } \\
\text { Principal }\end{array}$ & 207.4 & 309.87 & 236.5 & 111.2 & 19.2 & 229.1 & 14.2 & 804.1 & 655.35 & 589.66 \\
\hline $\begin{array}{c}\text { Maximum } \\
\text { Shear }\end{array}$ & 206 & 311.59 & 237.5 & 102.1 & 19.2 & 241.8 & 14.2 & 842.3 & 666.31 & 564.95 \\
\hline Von Mises & 192.9 & 328.79 & 245.8 & 106.4 & 18.5 & 244.8 & 14.9 & 827.8 & 662.57 & 581.38 \\
\hline
\end{tabular}

The improvement in mass's boundary preservation can be explained by the fact that those strains were derived from the normal and shear axial strains, so both strains play their role in the elasticity imaging process. The axial strain provides contrast in the direction of the mechanical compression, while the axial shear provides contrast with an angle relative to the direction of the mechanical compression, and that angle depends on the amount of exhibited shear strain. Shear slip mechanism at the boundaries or interfaces between dissimilar stiff and soft tissues was the main reason for the potential mass's boundary enhancement. As in the phantom study, the derived strains showed the inclusion and inclusion background border with different degrees of clarity.

Interactive and automatic segmentation algorithms based on active contour methods [77, 118] can be optimized using the maximum shear elastography. Those types of segmentation algorithms have the limitation of requiring the masses to be relatively regular with well-circumscribed single margins on the images, which is not the case with breast masses. This limitation can be overcome by using the new derived elastography strains, as the boundaries become more profound in the resulting images and volumes. 


\subsection{Study Summary}

In vivo 3D elastography study was demonstrated, and its ability to better diagnose stiff masses inside soft tissues was illustrated. A new breast mass classification method based on a nonlinear material behavior was investigated and validated. The method was tested on human subjects in vivo and outcomes were successful to correctly diagnose the cases involved in the study, and that was verified with the biopsy results. The initial results are very promising and significant. The use of the new nonlinear parameters may increase the overall sensitivity and specificity rates of elastography techniques in classifying breast masses, and hence limiting the number of unnecessary biopsy procedures. Using relative stiffness for classification suffered from a large overlap in values between benign and malignant cases. Relative mass volume proved to be a good classification parameter. Yet, the mass needs to be well bounded and very clear in the B-mode images to get an accurate diagnosis, which is not always the case for breast masses.

In addition, new elastography volumetric strain types were introduced, and there usage in emphasizing the stiff mass's boundary was presented. This can lead to improve the overall performance rates of automatic segmentation algorithms, as will be shown in the next chapter. 


\section{CHAPTER 6}

\section{BREAST MASSES SEGMENTATION}

In this chapter, the segmentation algorithms are applied and tested on different elastographic data sets. Gradient vector flow (GVF) and distance regularized level set (DRLSE) evolution segmentation methods were considered. The two algorithms were first evaluated for appropriateness to segment elastography images, and the best performing method is selected for further analysis. Based on the segmented volumetric elastography, the breast mass's shape and approximate size are determined. Moreover, the boundary enhancement effect of the derived elastography strains was verified by comparing the segmentation performance for each strain type. As will be discussed, this advanced visualization method has demonstrated its value as a powerful three dimensional characterization technique.

\subsection{Applying Segmentation}

This phase of research is concerned with applying two segmentation algorithms on the constructed elastograms and selecting the optimal algorithm for further constructions. Algorithm evaluation was first performed on the in vitro breast phantom volumetric data. As described in chapter 3, volumetric segmentation is performed on a slice by slice basis. Using either one of the two segmentation methods; GVF snake or DRLSE, the following procedure was followed. All slices of the raw elastography volume are first displayed by the MATLAB program. The raw volume is the elastography volume that axial-elevational scan conversion was not applied on. This was for the purpose of minimizing the number of slices needed to be reviewed and selected by the user, and then scan conversion can be easily performed on the segmented slices. For each slice the program prompts the operator to decide whether the currently shown slice contains the stiff inclusion (mass) or not. If the current slice shows the mass, the user is then prompted to select a single point inside the mass's area. The user could have chosen an area inside the inclusion's area, yet because of the shape's variability of the in vitro inclusions and in vivo masses, a multipoint selection is necessary. This task would have been an additional burden on the operator, and the selection of only one point would be more user friendly, where the algorithm can continue from 
there. The selected point is used by the program to create an initial inclusion's segmentation contour. The program proceeds by evolving the initial contour for a number of specified iterations, and the final delineating mass contour is stored for each slice.

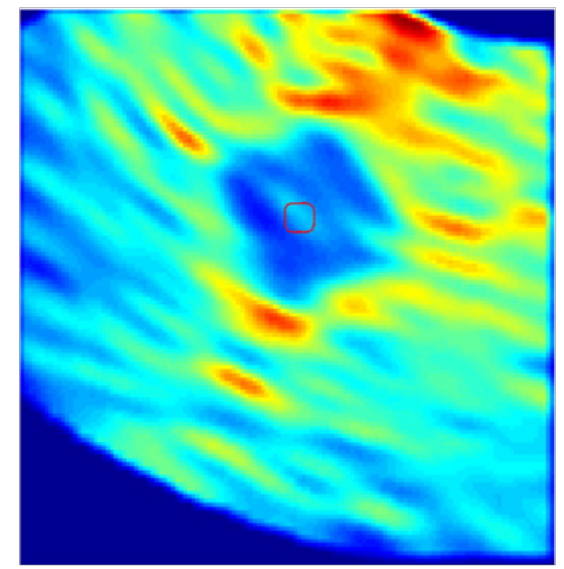

a)

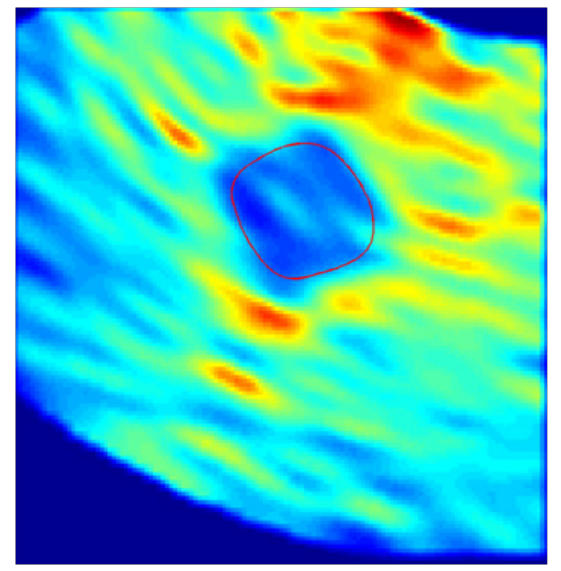

c)

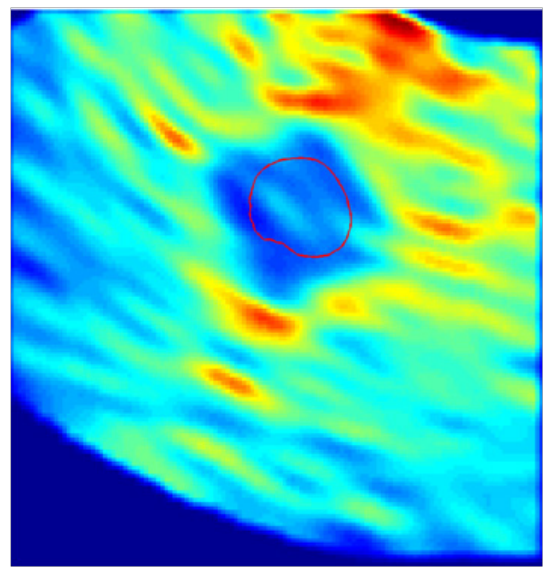

b)

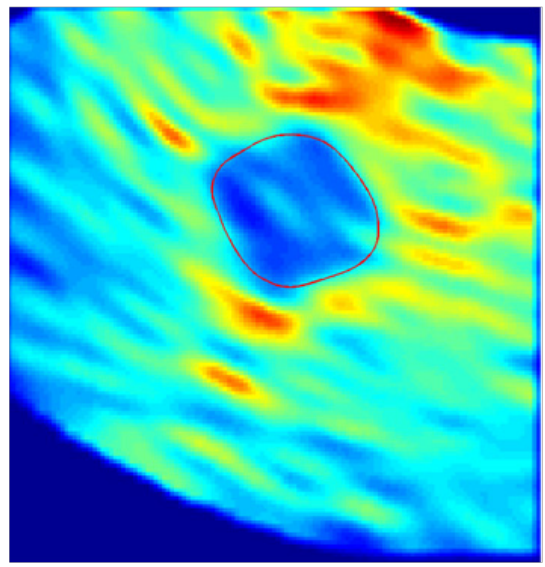

d)

Figure 6.1 Example curve evolution at different iteration stages: a) initial contour, b) 60 iterations, c) 120 iterations d) 200 iterations.

An example of progression of the contour's evolution inside the phantom's stiff inclusion is demonstrated in Fig. 6.1. The square initial contour is shown in Fig. 6.1 a, the operator selected a point near the center of the stiff area and the program created an initial kernel of 6X6 pixels around the selected point. As the segmentation algorithm solves the governing partial differential equations, the evolving contour starts to reshape and expand towards the inclusion's boundary. The more computed iterations, the more convergence the contour achieves, until the final contour is 
reached where no more evolution is needed. In the shown image, we can see the contour evolved very fast between iterations 1, 60 and 120, while the evolution between 120 and 200 was minimal, as the outlining contour has almost reached the desired location. Both segmentation algorithms had very similar performance for simple images as shown in Fig. 6.1, yet for more complex topologies the GVF snake algorithm faces some difficulties during the contour evolution.

\subsection{Evaluating Segmentation Algorithms}

The originally set criteria for evaluating the two segmentation algorithms using the breast phantom were the followings:

1. Compare the performance on simple phantom inclusion's $2 \mathrm{D}$ elastograms, and check if the whole targeted areas were delineated effectively by the algorithm.

2. Compare the performance on the phantom's 3D elastograms, and if that was successful then

3. The accuracy of the inclusion's calculated volume, relative to the true volume specified in the phantom's datasheet.

As shown in the last subsection, both algorithms were performing with similar efficiencies while applied to simple 2D elastograms. But, when it was attempted to segment the phantom's 3D elastograms, the GVF snake algorithm failed to segment some slices that showed a degree of complex topologies. The DRLSE method continued to successfully delineate the inclusion in all slices, and ultimately the 3D segmented volume was constructible. Fig. $6.2 \mathrm{a}-\mathrm{b}$ shows the results of the two algorithms after the final contour was reached and overlaid on the original elastogram. Both algorithms were set to solve at the same number of iterations, and segmentation parameters were optimized to achieve the best segmentation within each algorithm. The shown slice exhibits a degree of nonuniformity inside the area corresponding to the inclusion, wherein a hill with relatively higher strain values appears across the inclusion. The DRLSE method succeeded to overcome this topology change, and the contour evolved to the desired location with reasonable accuracy. On the other hand, the GVF method could not overcome this topological disturbance, and the resulting contour was intersected on itself.

Another example showing the methods comparison is presented in Fig. $6.2 \mathrm{c}-\mathrm{d}$. An in vivo breast mass segmentation was attempted using the two algorithms. Once more, the DRLSE effectively delineated the stiff breast mass. However, the GVF algorithm could not handle the complex image topologies of this in vivo image. At low number of iterations, the GVF did not 
stretch the contour to cover the area corresponding to the stiff mass, especially the right and left edges. In attempt to enhance the contour's evolution, the number of iterations was increased to 250 iterations, so that the contour would spread more towards the boundaries. Yet, when the contour was expanding, it penetrated the upper and lower mass boundaries; an effect famously known as boundary leakage.

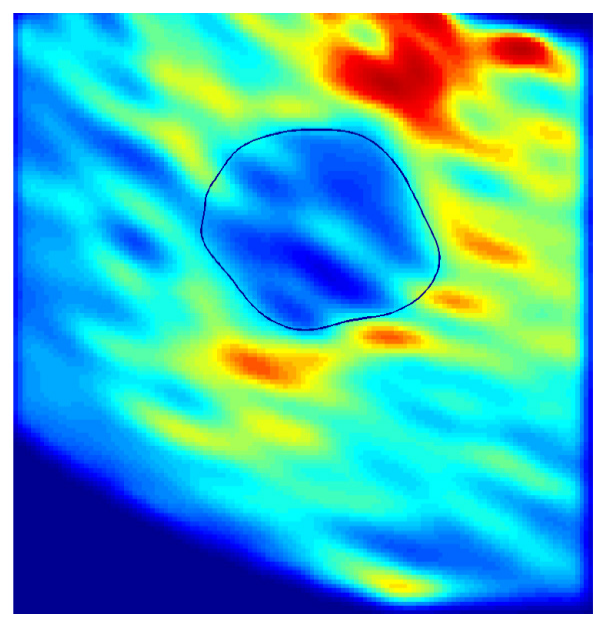

a)

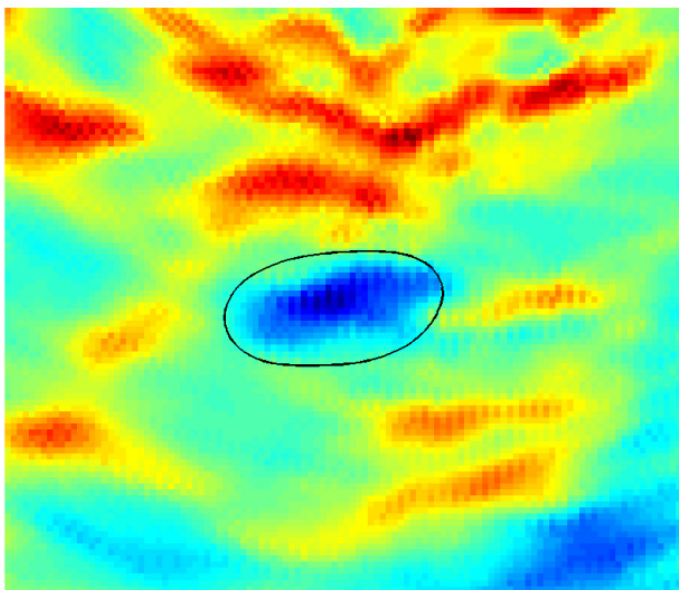

c)

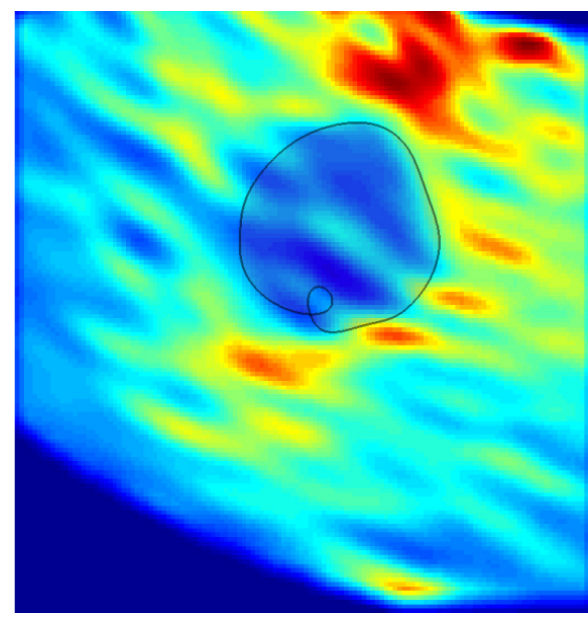

b)

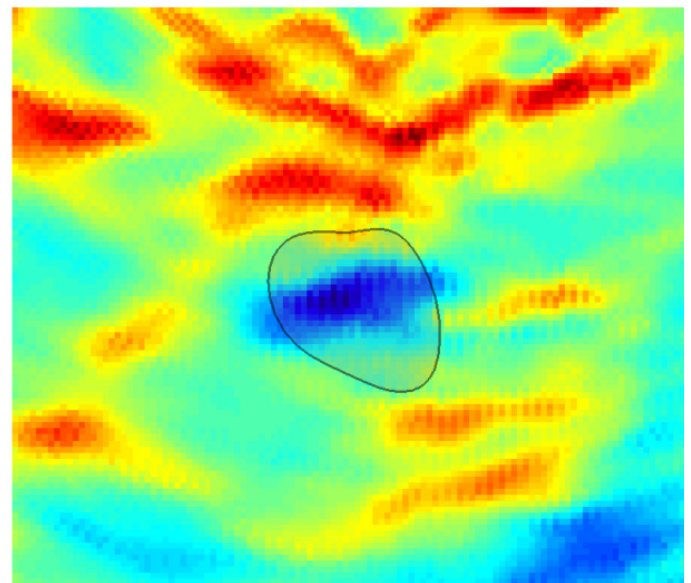

d)

Figure 6.2 Segmentation performance comparison between the two segmentation methods. a-b) Phantom's inclusion segmented using DRLSE and GVF snake, respectively. c-d) In vivo breast mass segmented using DRLSE and GVF snake, respectively. a) and c) shows better segmentation using the DRLSE method. 
It is worth mentioning that the DRLSE can only suffer from the boundary leakage effect, when the wrong segmentation parameters are selected. This downside can be avoided by carefully setting the parameters. However, through the whole study this method did not experience the contour self intersection effect, which the GVF snake algorithm has shown.

In summary, these segmentation evaluations show that the DRLSE level set method can perform more effectively than the GVF snake method, which experienced difficulties to segment specific images. The level set method did not suffer from contour self intersection, and the boundary leakage effect was found to be controllable through careful parameter selection. Therefore, the rest of the elastographic in vitro and in vivo data were segmented and analyzed using the DRLSE segmentation algorithm.

\subsection{D In Vitro Segmentation}

At this point, the application of segmentation was expanded to 3D. Individual slices of the corresponding 3D elastogram were segmented using the DRLSE method, and then scan converted in the axial-elevational plane to produce isolated volumetric masses. The phantom's inclusion was first segmented from the four volumetric elastograms; normal axial, first principal, maximum shear and Von Mises strains. Parameters of the DRLSE segmentation algorithm were fixed for the four volumetric strains. The main segmentation parameters were set as follows:

- Gaussian filter: kernel size $=12$ X 12 pixels, and standard deviation $=5$.

- Evolution equation weights: $\alpha=-3, \lambda=5$, and $\mu=0.2$

Delineated 3D views of the inclusion are presented in Fig. 6.3 a-d. The inclusion's shape in the four strain volumes appears very close to the spherical shape, which is the true shape of the phantom's inclusions. The variations between the four volumes were insignificant, with the exception of the normal axial strain being slightly deformed. This is because the normal axial strain exhibits the weakest mass boundary among the used volumetric strains, as emphasized in Figs 4.4 and 4.5. Moreover, the inclusion's volume was automatically computed based on the segmented 3D elastograms in the four cases, and listed in Table 6.1, along with the associated volume calculation errors. The actual diameter of the inclusion was $9 \mathrm{~mm}$ as measured from the B-mode image, which facilitated calculation of the true inclusion's volume. 


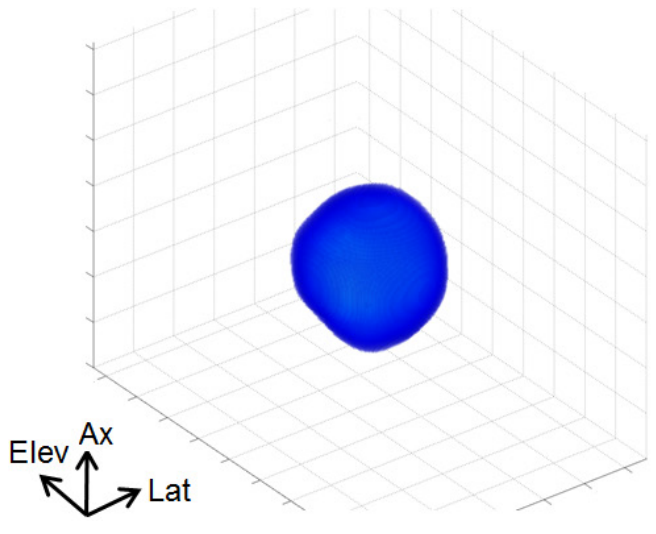

a)

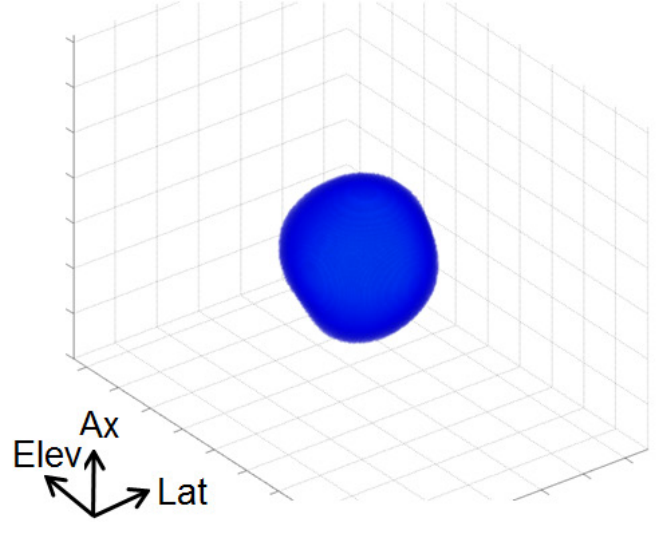

c)

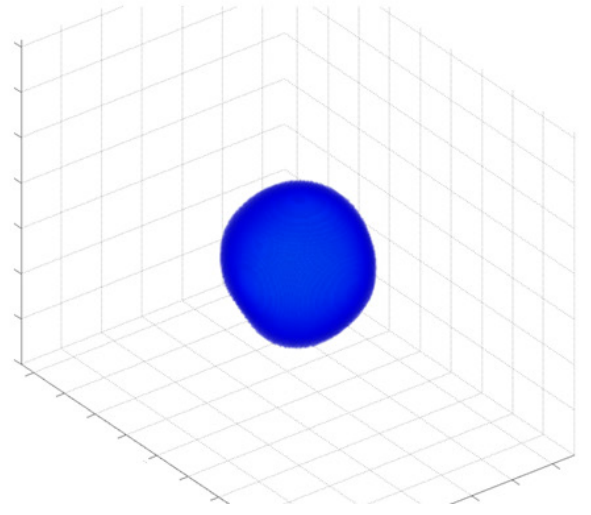

b)

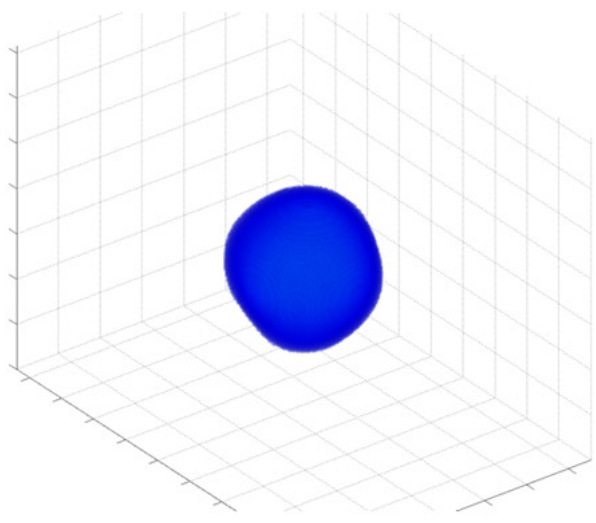

d)

Figure 6.3 Segmented derived strain elastograms for the breast phantom: a) normal axial strain, b) first principal strain, c) maximum shear strain d) Von Mises strain.

Elastographic volume calculations were noticed to agree well with the actual size. In terms of the absolute error, the maximum shear elastography provided the closest volume estimation to the actual size. The stronger inclusion's boundary exhibited by the 3D maximum shear elastogram restricted any possible boundary leakage during the segmentation's contour evolution. Due to the fairly homogeneous breast phantom, the resultant image quality was high, and the inclusion was already well bounded and distinct from the soft tissue in the four elastographic strains. Thus, the shape difference was insignificant between the four segmented volumes. However, for the in vivo imaging this was not the case, and segmentation results were different, as will be shown in the following subsections. 
Table 6.1 Phantom's inclusion volume estimations using interactive segmentations.

\begin{tabular}{ccc}
\hline & Calc. Volume $\left(\mathrm{mm}^{3}\right)$ & Error \\
\hline Actual size & 381.704 & \\
\hline Axial Strain & 397.664 & $\mathbf{4 . 1 8 \%}$ \\
\hline First Principal & 372.070 & $\mathbf{- 2 . 5 2 \%}$ \\
\hline $\begin{array}{c}\text { Maximum } \\
\text { Shear }\end{array}$ & 373.584 & $-\mathbf{2 . 1 3 \%}$ \\
\hline Von Mises & 369.456 & $\mathbf{- 3 . 2 1 \%}$ \\
\hline
\end{tabular}

\subsection{D In Vivo Segmentation}

\subsubsection{Normal Axial Strain Imaging}

The aforementioned 3D imaging methods were applied on the nine studied breast tumors. The main segmentation parameters were set as follows:

- $\quad$ Gaussian filter: average kernel size $=9 \times 9$ pixels, and standard deviation $=5$.

- Evolution equation weights: $\alpha=-3, \lambda=5$, and $\mu=0.2$.

These parameters were fixed for all cases, except the Gaussian kernel size that was set to $4 \mathrm{X} 4$ for two cases showing small and smooth masses, wherein only weak filtration was needed.

Figure 6.4 demonstrates the 3D imaging of the benign cases. The first column states the patient's age and pathological diagnosis using biopsy, second column shows the normal axial strain 3D elastography, third column shows the corresponding segmented 3D elastography, and the fourth column states the mass's estimated volume based on the segmented volumes. Transparency was applied whenever possible on the 3D elastograms, to better show the masses inside the corresponding volumes. Transparency effect was achieved by hiding the high strain voxel values (soft tissue) and showing only the low strain voxel values (stiff inclusions). Malignant tumors 3D imaging results are also demonstrated in Fig. 6.5. Based on these figures, the potential of using 3D elastography along with its segmented version is very evident. Mass shape and volume estimations 
provided a clear insight on the extent of the tumor in each case. Furthermore, small tumor sizes down to about $15 \mathrm{~mm}^{3}$ were also detectable and measureable using the presented 3D imaging techniques.

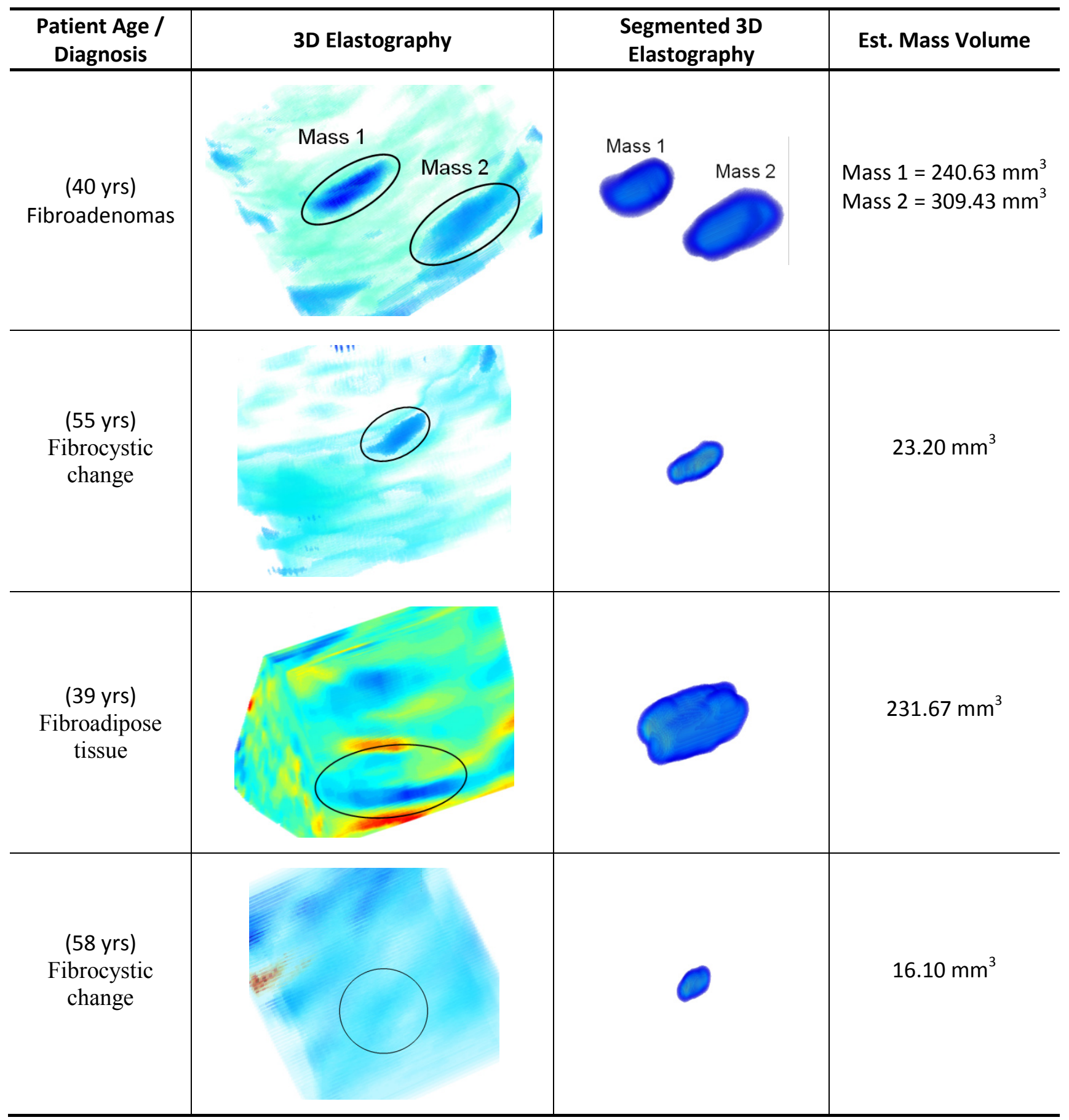

Figure 6.4 Summary of the benign mass's characterization: first column states the patient's age and biopsy diagnosis, second column shows 3D axial elastography, third column shows segmented 3D axial elastography, and fourth column states the estimated mass's volume. 


\begin{tabular}{|c|c|c|c|}
\hline $\begin{array}{c}\text { Patient Age / } \\
\text { Diagnosis }\end{array}$ & 3D Elastography & $\begin{array}{c}\text { Segmented 3D } \\
\text { Elastography }\end{array}$ & $\begin{array}{c}\text { Est. Mass } \\
\text { Volume }\end{array}$ \\
\hline $\begin{array}{c}\text { (46 yrs) } \\
\text { Invasive ductal } \\
\text { carcinoma }\end{array}$ & & & $235.10 \mathrm{~mm}^{3}$ \\
\hline $\begin{array}{c}\text { (56 yrs) } \\
\text { Invasive lobular } \\
\text { carcinoma }\end{array}$ & & & $117.90 \mathrm{~mm}^{3}$ \\
\hline $\begin{array}{c}\text { (46 yrs) } \\
\text { Invasive ductal } \\
\text { carcinoma }\end{array}$ & & & $821.40 \mathrm{~mm}^{3}$ \\
\hline $\begin{array}{c}\text { (77 yrs) } \\
\text { Invasive ductal } \\
\text { carcinoma }\end{array}$ & & & $658.20 \mathrm{~mm}^{3}$ \\
\hline $\begin{array}{c}\text { (65 yrs) } \\
\text { Invasive ductal } \\
\text { carcinoma }\end{array}$ & & & $624.60 \mathrm{~mm}^{3}$ \\
\hline
\end{tabular}

Figure 6.5 Summary of the malignant mass's characterization: first column states the patient's age and biopsy diagnosis, second column shows 3D axial elastography, third column shows segmented 3D axial elastography, and fourth column states the estimated mass's volume. 
To evaluate the overall clinical efficacy of the segmented volumetric breast masses, an experienced radiologist provided quality rating for each segmented breast mass. The radiologist took into consideration the appearance of the tumor margins, and the smoothness degree of the constructed volumes. In general, images portraying smooth masses would indicate benignancy, while angular mass margins help the radiologist to decide if a mass is malignant. Those features help the radiologist determining the mass diagnosis. The rating depended also on other factors reflecting the expected shape using multi-planar mammography and conventional B-mode ultrasound images. Rating score ranged from $0=$ "unacceptable" to $10=$ "Excellent". Figure 6.6 shows a histogram of the clinical segmentation performance ratings for the ten segmented breast masses.

The clinical evaluation of the segmented breast masses is a standard assessment method for various imaging modalities. The segmented volumes showed that most of the studied masses depicted features that reflect their diagnosis, and also the expected shape from mammography and ultrasound imaging modalities. Lower ratings of 7 and 8 were given specifically to volumes showing malignant masses while their appearance was either smoother than expected, or not angular in margins. Yet, the overall rating was about 9 out of 10, which indicates that the produced outlined volumes represent a useful diagnostic imaging tool.

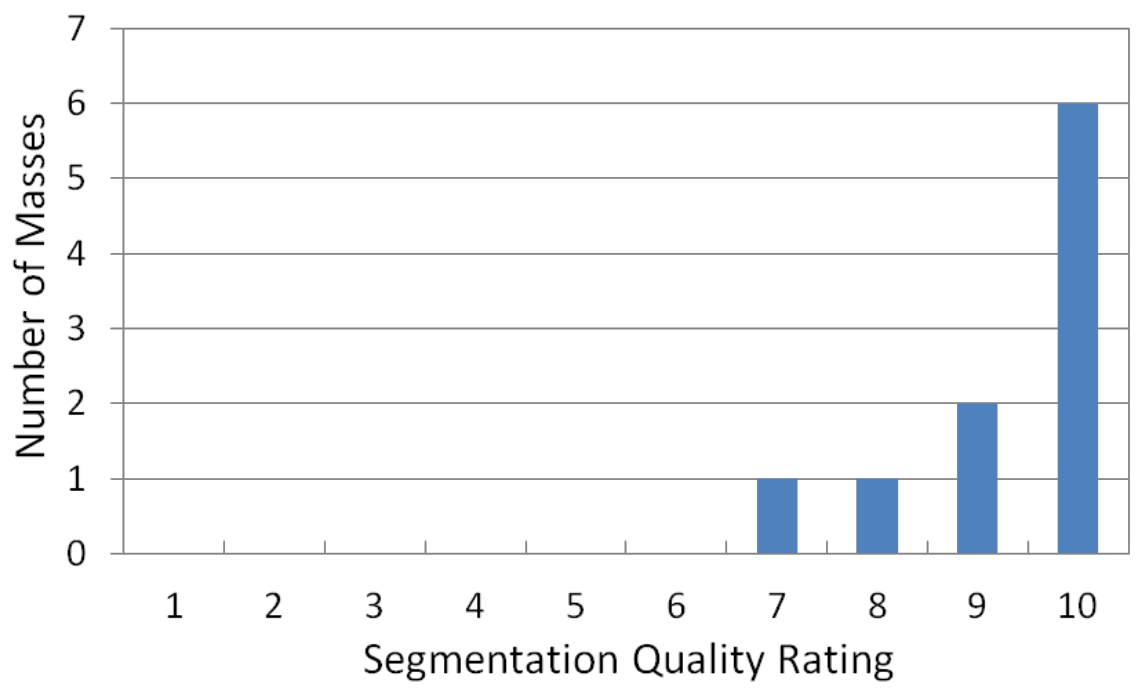

Figure 6.6 Mass segmentation performance evaluated by an experienced radiologist. 


\subsubsection{Derived Strains Effect}

The effect of the derived strains; as defined by Eq. 3.5 on the segmentation performance of in vivo data is now demonstrated. Segmentation algorithm parameters were fixed for the normal axial elastogram and the three derived strains elastograms, for a fair performance comparison. Figure 6.7 displays the four elastographic volumes for one patient having two breast masses, diagnosed with biopsy as benign masses; fibroadenoma. This patient was the same case shown in Fig 5.1. From Fig 6.7, the segmented volumes show a clear outline of the two masses. The volumetric normal axial elastogram shows some deformation when compared to the other three volumetric derived strains, especially mass number 1 .

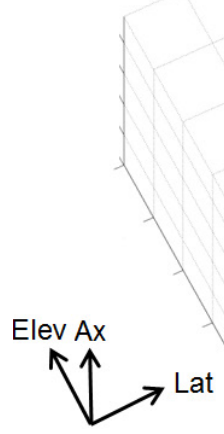

Mass 1

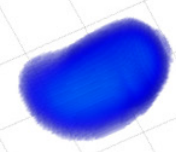

a)
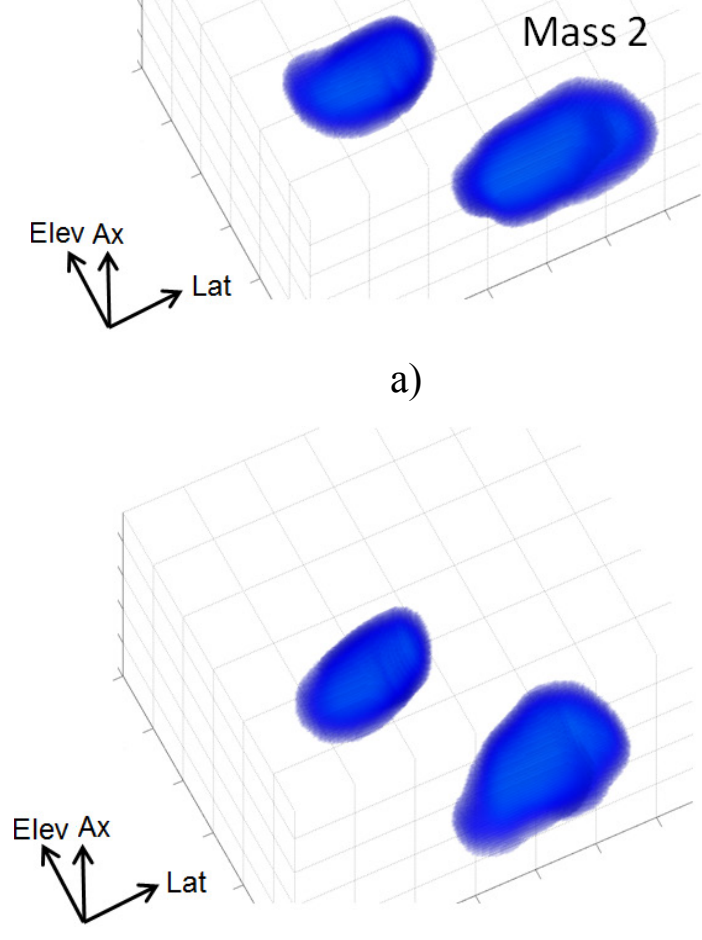

c)

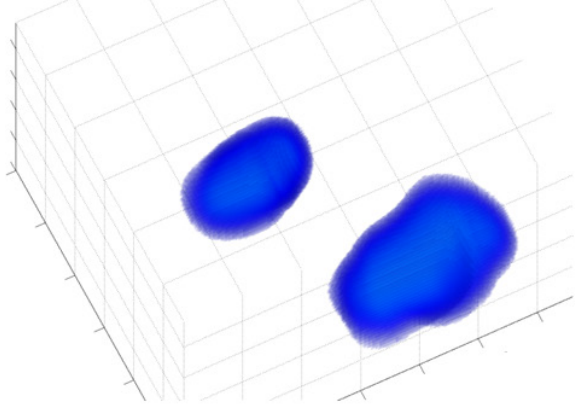

b)

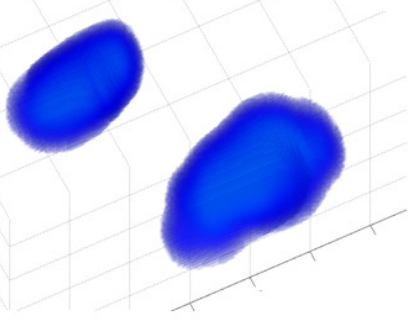

d)

Figure 6.7 Segmented derived volumetric strain elastograms of a benign case; fibroadenoma. Patient's age was 40 years. a) normal axial strain, b) first principal strain, c) maximum shear strain , d) Von Mises strain. 


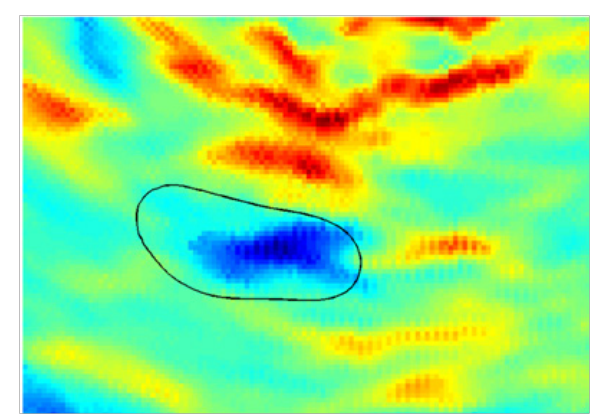

a)

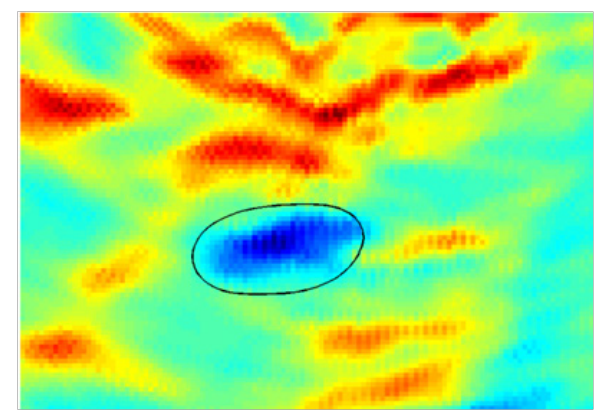

c)

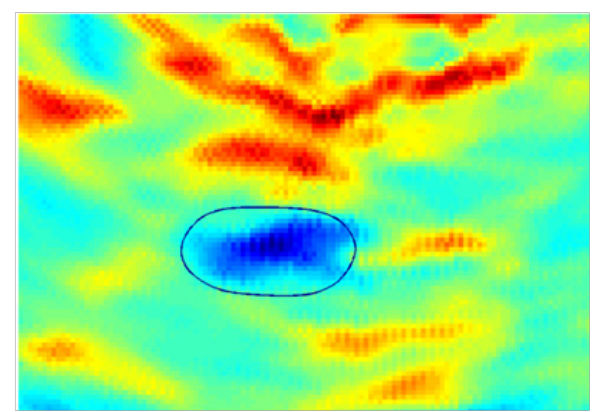

b)

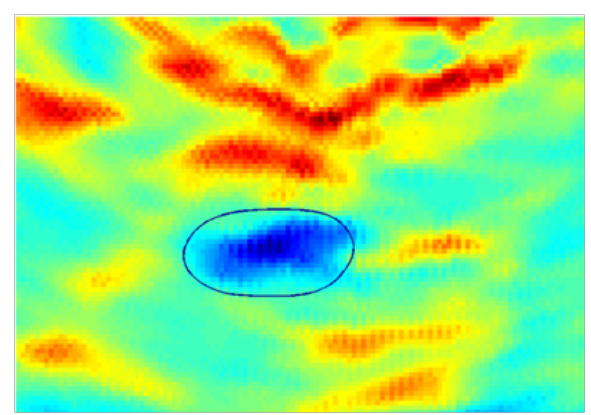

d)

Figure 6.8 Segmented strain elastograms corresponding to mass 1 in Fig. 9: a) normal axial strain, the segmentation contour shows some leakage beyond the mass's boundary, b) first principal strain, c) maximum shear strain d) Von Mises strain. No boundary leakage appears in the derived strains.

Mass shape deformation was the effect of boundary leakage that occurred during the segmentation, which was most significant for mass number 1, as illustrated in Fig. 6.8. The figure shows four slices of the volumetric elastograms corresponding to the outlined mass number 1 shown in Fig. 6.7. Automatic segmentation of the normal axial strain slice showed some boundary leakage beyond the mass's upper left edge. Because of the faint edge between the stiff mass and the surrounding soft tissue, the evolving contour leaked from its desired location. While in the other three elastograms, the evolving contour did not leak beyond the desired location, as a result of the enhanced boundary of the derived strains. It is noticeable from Fig. 6.8 the degree of enhancement of the mass's boundary, especially in the maximum shear elastogram.

Figure 6.9 shows another segmented 3D elastograms for another case, with their corresponding mass sizes. Mammography showed an irregular mass at 8:00 in the right breast. Biopsy revealed breast cancer being invasive ductal carcinoma, mixed with some fibroadenomal tissue. The four volumes showed different outlines of the breast mass segmented using fixed segmentation parameters. The normal axial volume displayed two apexes in the axial direction; the $\mathrm{Y}$ axis on the 
figure. First principal volume showed similar apexes, but with a much smaller secondary apex. While, both the maximum shear and Von Mises volumetric strains almost did not show this secondary apex. The reason behind this is illustrated in Fig. 6.10, in which a segmented slice of each type of the volumetric strains is shown. Figure 6.10 a) shows a clear boundary leakage that occurred during the contour's evolution, because of the weak separation between the stiff mass and the surrounding healthy tissue. This effect had a much lower intensity in the first principal slice; Fig. $6.10 \mathrm{~b}$ ), as a small amount of boundary leakage occurred. The maximum shear slice did not show any boundary leakage, and the Von Mises strain showed an insignificant amount of leakage, as shown in Fig. $6.10 \mathrm{c}-\mathrm{d}$ ). This effect was also reflected in the calculated mass's volumes, in which the axial normal strain revealed the largest estimated size, due to the existence of the false secondary apex.
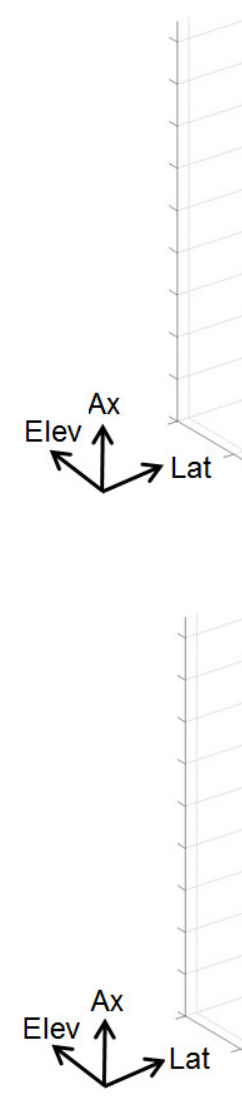

a)

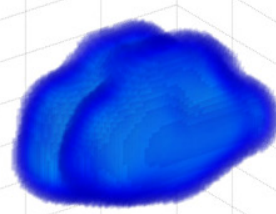

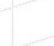
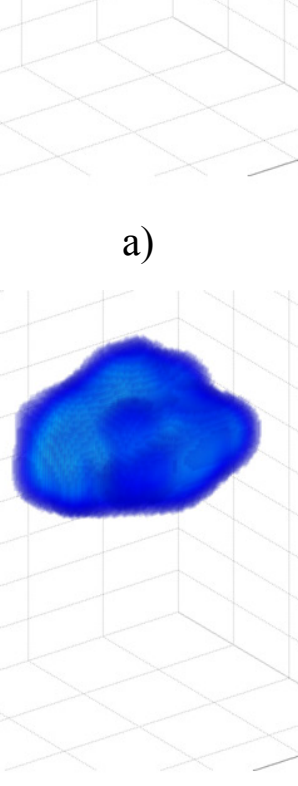

c)

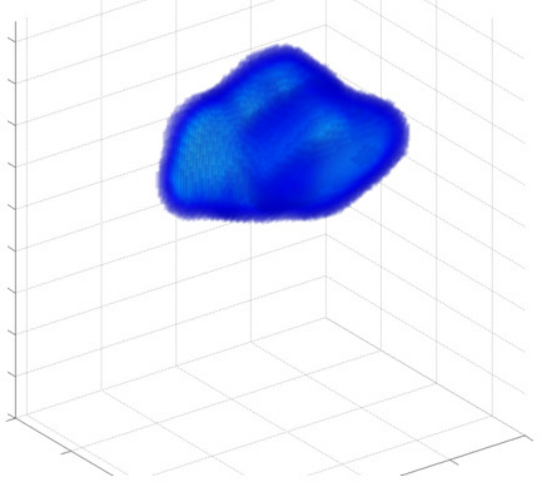

b)

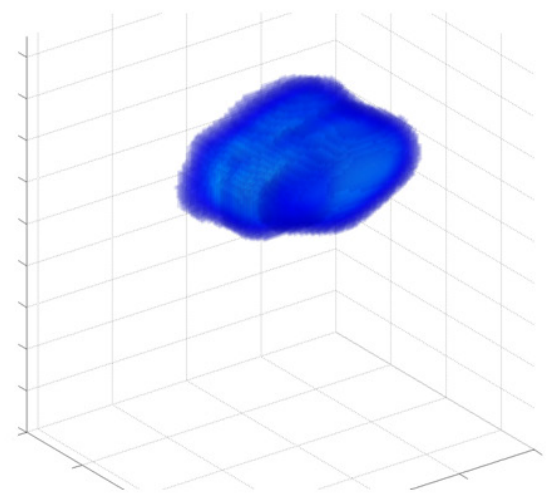

d)

Figure 6.9 Segmented derived volumetric elastograms of a malignant mass; IDC. Patient's age was 65 years. a) normal axial strain; estimated volume: $624.60 \mathrm{~mm}^{3}$, b) first principal strain; estimated volume: $609.10 \mathrm{~mm}^{3}$, c) maximum shear strain; estimated volume: $602.90 \mathrm{~mm}^{3}$, d) Von Mises strain; estimated volume: $592.40 \mathrm{~mm}^{3}$. 


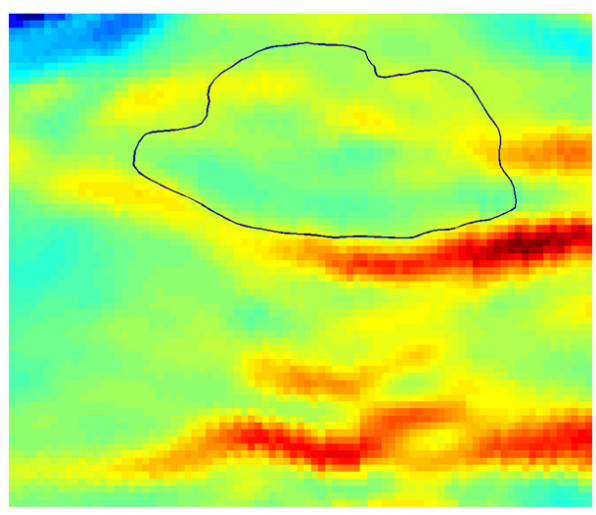

a)

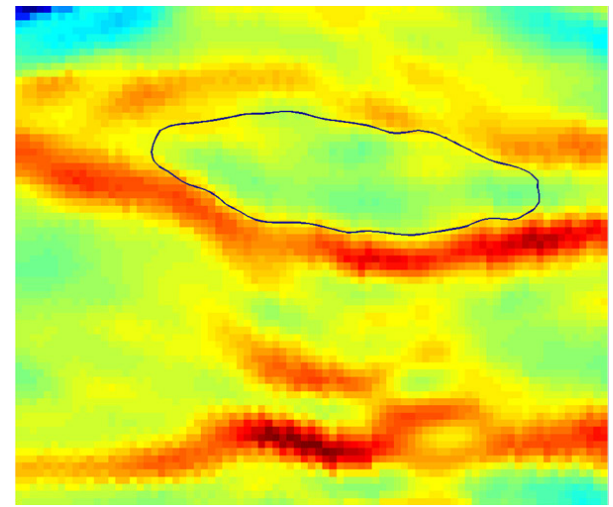

c)

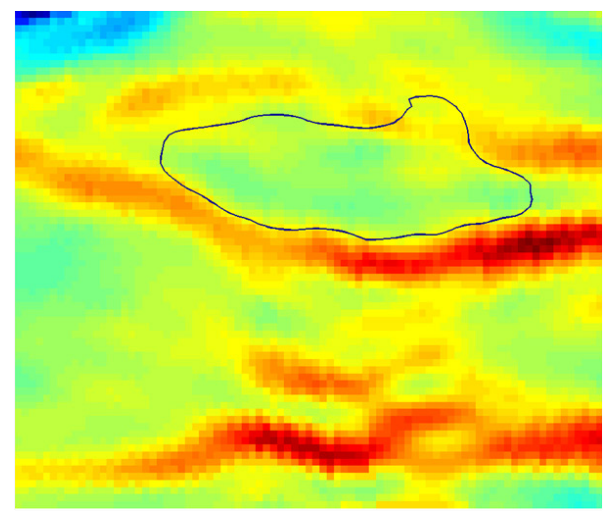

b)

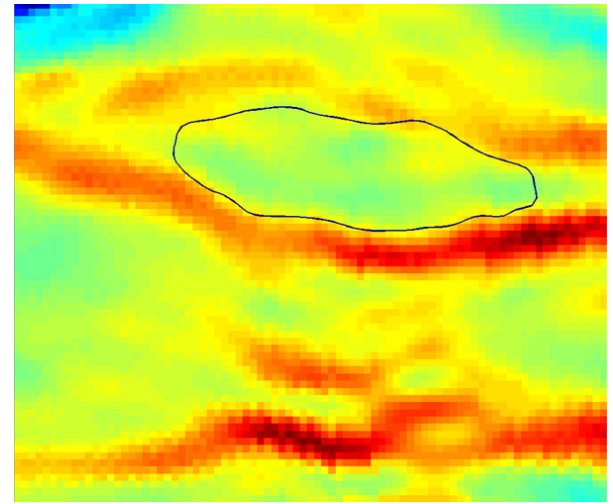

d)

Figure 6.10 Segmented strain elastograms corresponding to the mass in Fig. 11: a) normal axial strain, the segmentation contour shows some leakage beyond the mass boundary, b) first principal strain, a minor degree of boundary leakage appears, c) maximum shear strain d) Von Mises strain. No boundary leakage appears in the two latter strains.

Additionally, the sizes of the ten examined breast masses were calculated based on the segmented volumes, as listed in Table 6.2. Generally, it can be noted that the calculated volumes using the normal axial elastogram were larger than the volumes calculated using the derived strains. This general observation can be brought back to the improved mass's boundary in the three derived strains, wherein the segmentation algorithms was more constrained by the mass's edges. 
Table 6.2 Breast mass volume $\left(\mathrm{mm}^{3}\right)$ calculations for all cases using interactive segmentation.

\begin{tabular}{c|cccccccccc}
\hline $\begin{array}{c}\text { Mass } \\
\text { Number }\end{array}$ & $\mathbf{1 - 1}$ & $\mathbf{1 - 2}$ & $\mathbf{2}$ & $\mathbf{4}$ & $\mathbf{5}$ & $\mathbf{6}$ & $\mathbf{7}$ & $\mathbf{8}$ & $\mathbf{9}$ & $\mathbf{1 0}$ \\
\hline Axial Strain & 240.63 & 309.43 & 235.10 & 117.90 & 23.20 & 231.67 & 15.30 & 821.4 & 658.29 & 624.60 \\
\hline $\begin{array}{c}\text { First } \\
\text { Principal }\end{array}$ & 225.90 & 301.30 & 230.86 & 113.38 & 21.75 & 221.10 & 16.10 & 799.07 & 663.20 & 609.10 \\
\hline $\begin{array}{c}\text { Maximum } \\
\text { Shear }\end{array}$ & 227.36 & 298.56 & 230.58 & 112.3 & 20.43 & 238.61 & 13.70 & 792.73 & 668.31 & 602.90 \\
\hline Von Mises & 220.53 & 297.50 & 227.98 & 112.53 & 21.25 & 236.89 & 13.22 & 787.33 & 666.75 & 592.40 \\
\hline
\end{tabular}

In conclusion, the segmented in vitro volumes were useful to examine the presented imaging methods and display the delineated inclusion in 3D, although the segmented derived strains provided a slight improvement over the traditional normal axial strain, regarding the estimated inclusion shape and size. However, when the methods were applied on human breast masses, a noticeable improvement was observed. The segmentation algorithm performed more efficiently for some masses using the derived strains, which offered a more accurate mass segmentation, by avoiding contour boundary leakage.

The reason behind this improvement for the breast masses is the degree of image quality of the constructed elastogram, which is generally lower for in vivo experiments. The material of the breast phantom is fairly homogenous and the resultant images showed well bounded inclusions using the four strain types, which resulted in almost equal segmentation performance for the four strain types. On the other side, human breast tissue is inhomogeneous and the constructed elastograms might not show well circumscribed tumor margins, which affects the segmentation process. The main goal of using the derived strains; especially the maximum shear strain, is to improve the overall segmentation performance by emphasizing the tumor margins and avoid boundary leakage, which ultimately preserved the final constructed shape of the tumor. It is also worth mentioning that not all the examined breast masses experienced the same degree of improvement in the segmentation process using the derived strains. About one third of the examined masses exhibited this kind of improvement, which is still a significant ratio. In addition, mass volume estimations using different strain types were very close to each other, except for the cases that experienced boundary leakage, where the normal axial strain provided an overestimated volume. 


\section{CHAPTER 7}

\section{CONCLUSIONS AND FUTURE WORK}

\subsection{Concluding Remarks}

The research presented in this dissertation aims to noninvasively diagnose breast tumors weather being benign or malignant. Breast cancer is now considered a lethal disease that is spreading and continuously affecting people worldwide. Therefore, early cancer diagnosis is essential to increase the survival rate of the diseased patients, and also to avoid tumor metastasis. Several clinical factors are considered to predict the stage of the cancer and the chances of successful treatment plans, thus providing accurate prognosis of the tumor. These factors include the tumor's shape and size [119]. Tumors with indistinct and angular margins would indicate malignant tumors, while those with well defined margins indicate benign tumors. In addition, tumors with larger sizes are associated with a worse prognosis and pose higher risk than smaller tumors. Current diagnostic methods that are used for diagnosis of breast tumors have some disadvantages. In general, mammography imaging followed by pathological diagnosis using biopsy is routinely performed to diagnose the suspected tumors. Mammography has high sensitivity for breast cancer detection, and it has improved the survival rate of women suffering from breast cancer. The main disadvantage of mammography is the decreased sens

itivity for females with dense breasts. Biopsy is routinely performed under conventional ultrasound guidance, yet about $80 \%$ of the performed biopsies yield a benign diagnosis [43, 120, 121]. In addition to causing much stress for the patient, biopsy is also an invasive and costly procedure.

Potential characterization methods for breast tumors using ultrasound elastography have been illustrated. From the demonstrated results, it has been shown that useful information about the breast tumor can be predetermined noninvasively. Classification of breast tumors was achieved using a new method employing the tumor's mechanical nonlinearity. The integration between ultrasound and the mammography stage provided a means to deliver controlled tissue multicompression that was necessary to apply the proposed classification method. The combined system 
provided the advantage of applying our new methods in a readily established clinical environment. A modified strain imaging algorithm was used to construct the strain images, by tracking temporally windowed radio-frequency signals. The new key features of the used algorithm were using direct signal interpolation before cross-correlation, and using spline smoothing functions on the estimated displacement maps. Both operations provided an enhanced image quality and reduced the effects of random noises. It is well known that 3D imaging is advantageous over 2D imaging as it convey information about the tissue in the elevational dimension as well as the axial and lateral dimensions. 3D volumes provide multi-readings per acquisition, which ultimately improves the overall accuracy of the mass characterization process.

Currently, most of the classification methods are based on a linear biomechanical tissue model. The proposed mass classification method was based on a nonlinear model for the tissue under investigation. The estimated strain differences between the breast mass and the surrounding soft tissue were observed over different pre-compression levels. The parameters describing the nonlinear (or linear) behavior of the tissue were utilized in the characterization process. The discrimination margin between the main two mass types was found to be larger using the proposed approach, and statistically supersedes the currently available conventional elastography methods.

Additionally, derived strains were exploited in constructing new elastographic imaging types. They provided a visual improvement to the examined breast mass. The derived strain used were the first principal, Von Mises and maximum shear strains, with the latter providing the highest degree of boundary enhancement. Segmentation techniques based on active contour methods was optimized using the maximum shear elastography. The use of automated segmentation algorithms provided an emphasize on the outline of the suspected breast mass from the surrounding healthy tissue: The main advantage of the implemented active contour methods is the ability to handle complex contours and sharp changes in the images topology, as in the case with breast elastography images. Three dimensional segmented images of the suspected masses provided a valuable means to predict the actual shape of the tumor. This information is valuable for many clinicians ad surgeons. Mass size was also determined through volume estimations, by exploiting the generated 3D mass segmentations. Segmentation quality assessment ratings showed that the outlining contours approximated many of the masses very well. 
The use of the proposed nonlinear imaging methods can help improve the overall efficiency of elastographic methods in classifying breast tumors. Other tissue types can also benefit from this classification method; such as prostate, liver and neck tumors, due to their inherent tissue nonlinearity. The presented techniques would not only make the breast examination process more cost and time effective, but also would potentially reduce the unnecessary performed biopsy examinations for the benign tumors. The prior knowledge of the tumors type, shape and size preoperatively, would provide very helpful information about the extent of the disease, which ultimately would help to decide the sort and the level of tumor treatment.

\subsection{Future Work}

The aforementioned methods have some limitations and challenging problems, which can be resolved in future work studies. Two main limitations are considered; the low number of multicompression points, and the small human sample size that was included. Throughout the in vivo study, the mammography stage's built-in force sensor was used to control the compression level. This sensor had a limited low resolution reading of $0.5 \mathrm{kgf}$, in which only measurements of integer force values were obtainable. This sensor enabled constructing force-strain plots using only a three multi-compression elastography measurements, taking into consideration the patient's safety of not exceeding $4 \mathrm{kgf}$ compression levels. To improve the accuracy of our nonlinear elastography technique, a force sensor with a resolution of $0.05 \mathrm{kgf}$ or better is required. The use of such sensor would ultimately lead to constructing force-strain curves with ten points or more, and hence achieve more accurate estimations of the nonlinear classification parameter. High resolution thin-sheet force sensors can be integrated easily with the already used custom compression ultrasoundmammography plates.

If we considered the method's sensitivity and specificity to detect breast cancer based on the studied human subjects, the numbers would be very high, which means that the method constitutes an almost perfect mass classifier. This study included 10 patients, and with this small sample size, the calculation of the corresponding sensitivity and specificity would not be so accurate. Therefore, a larger number of patients are required to calculate the true statistical parameters defining the new 
classification method's performance, and hence solidify our initial findings. Statistical analysis of the nonlinear classification method would be estimated based on an adequate sample size of at least 50 human subjects. The sample can include patients with benign and malignant breast masses, and healthy patients should be included as well. This is to ensure that the sample represents the real population of women suffering from breast masses. The actual efficiency of the new classification method can then be calculated, and enough data would be available to evaluate the true effectiveness of the proposed methods. In addition, commencing a new study of a large sample size would enable the creation of a database storing 3D elastography images associated with specific mass types. The database would also incorporate 3D imaging using different strain types that can provide enhanced visualization of the breast masses. This would help elucidate the relationship between the elastographic mass's appearances with its diagnosis. For example, the database would show how invasive ductal carcinomas would look like on 3D elastography, and what the expected nonlinear parameters values for such cases are.

The nonlinear classification of breast masses can be further verified using mechanical compression testing of the human tissue samples, which yields the true individual samples 'stressstrain curves. This would incorporate collecting fresh breast tumor samples for Lab mechanical testing at the same day. These curves will allow better understanding of the biomechanical tumor nonlinearity based on individual cases, rather than the generalized properties reported in the literature. Moreover, the results of the tests would facilitate a more accurate assessment of the $3 \mathrm{D}$ nonlinear elastography results, as the true mechanical properties for each examined tumor will be available. These in vitro tumor measurements can be further compared with the pathologic diagnosis of individual samples. This task is concerned with finding a correlation between each tumor diagnosis and the corresponding mechanical nonlinearity degrees, regarding the tumor's type, and the corresponding nuclear grade. The goal of such task is to discover a method to predict not only the type of the tumor, but also its stage and how sever it becomes.

It is well known that almost all biological tissue exhibit the property of anisotropy, and breast tumors are not an exception, especially that they contain a considerable amount of fibers. Investigating the tumor's anisotropy requires biaxial or even multi-axial biomechanical measurements. In order to apply the presented elastographic methods to study the tumor's anisotropy, at least one additional sensing probe should be employed in a direction perpendicular to 
the first probe, i.e. obtaining measurements in the axial and lateral directions. This procedure would require an even more sophisticated clinical setup, but this is beyond the scope of this work, and is considered an interesting and challenging research topic.

A custom nonlinear ultrasound diagnosis system that applies precise and controlled multicompressions directly on human subjects has been designed as a prototype. This system can be used to detect breast cancer as well as liver and neck cancer, by enabling examinations in the setting and laying patient's position. The system's implementation is considered as a long term goal, to develop a commercial elastography tumor classification system. 


\section{References}

[1] D. T. Ramsay, J. C. Kent, R. A. Hartmann, and P. E. Hartmann, "Anatomy of the lactating human breast redefined with ultrasound imaging," Journal of Anatomy, vol. 206, pp. 525534, Jun 2005.

[2] D. C. Pamplona and C. D. Alvim, "Breast reconstruction with expanders and implants: A numerical analysis," Artificial Organs, vol. 28, pp. 353-356, Apr 2004.

[3] N. Q. Nguyen, "Objective assessment of sonographic quality for breast cancer imaging," $\mathrm{PhD}$, Electrical and Computer Engineering, University of Illinois, 2012.

[4] P. J. Lynch. (2007, Breast Anatomy). Available: http://patricklynch.net/

[5] F. S. Azar, D. N. Metaxas, and M. D. Schnall, "A deformable finite element model of the breast for predicting mechanical deformations under external perturbations," Academic Radiology, vol. 8, pp. 965-975, Oct 2001.

[6] Gerard J. Tortora and S. R. Grabowski, Introduction to the human body, Fifth Edition ed. NY: John Wiley \& Sons, 2001.

[7] J. D. Sherman, Life's delicate balance: causes and prevention of breast cancer. London: Talyor and Francis, 2000.

[8] Z. Wang, "Mechanical and optical methods for breast cancer imaging," $\mathrm{PhD}$, Civil and Environmental Engineering, University of Iowa, 2010.

[9] B. Pasche, Cancer genetics. New York ; London: Springer, 2010.

[10] A. C. Miller. (2012, Breast abscess and masses. Available: http://emedicine.medscape.com/article/781116-overview

[11] Parris Wellman, Robert Howe, Edward Dalton, and K. A. Kern, "Breast tissue stiffness in compression is correlated to histological diagnosis," Harvard Biorobotics Laboratory1999.

[12] Y. C. Fung, Biomechanics : mechanical properties of living tissues, 2nd ed. New York ; London: Springer-Verlag, 1993.

[13] Michael Insana and M. Oelze, Advanced ultrasonic imaging techniques for breast cancer research IOS press, 2003.

[14] T. J. Hall, P. E. Barbone, A. A. Oberai, J. F. Jiang, J. F. Dord, S. Goenezen, and T. G. Fisher, "Recent results in nonlinear strain and modulus imaging," Current Medical Imaging Reviews, vol. 7, pp. 313-327, Nov 2011. 
[15] L. Pallwein, F. Aigner, R. Faschingbauer, E. Pallwein, G. Pinggera, G. Bartsch, G. Schaefer, P. Struve, and F. Frauscher, "Prostate cancer diagnosis: value of real-time elastography," Abdominal Imaging, vol. 33, pp. 729-735, Nov 2008.

[16] E. S. Burnside, T. J. Hall, A. M. Sommer, G. K. Hesley, G. A. Sisney, W. E. Svensson, J. P. Fine, J. J. Jiang, and N. J. Hangiandreou, "Differentiating benign from malignant solid breast masses with US strain imaging," Radiology, vol. 245, pp. 401-410, Nov 2007.

[17] G. Scaperrotta, C. Ferranti, C. Costa, L. Mariani, M. Marchesini, L. Suman, C. Folini, and S. Bergonzi, "Role of sonoelastography in non-palpable breast lesions," European Radiology, vol. 18, pp. 2381-2389, Nov 2008.

[18] T. A. Krouskop, T. M. Wheeler, F. Kallel, B. S. Garra, and T. Hall, "Elastic moduli of breast and prostate tissues under compression," Ultrasonic Imaging, vol. 20, pp. 260-274, Oct 1998.

[19] Jonathan Ophir, S. Kaisar Alam, Brian S. Garra, Faouzi Kallel, Elisa E. Konofagou, Thomas Krouskop, Christopher R. B. Merritt, Raffaella Righettr, Remi Souchon, Seshadri Srinivasan, and T. Varghese, "Elastography: Imaging the elastic properties of soft tissues with ultrasound," J Med Ultrasonics, vol. 29, pp. 155-171, 2002.

[20] C. J. Allegra, D. R. Aberle, P. Ganschow, S. M. Hahn, C. N. Lee, S. Millon-Underwood, M. C. Pike, S. D. Reed, A. F. Saftlas, S. A. Scarvalone, A. M. Schwartz, C. Slomski, G. Yothers, and R. Zon, "National institutes of health state-of-the-science conference statement: diagnosis and management of ductal carcinoma in situ september 22-24, 2009," Journal of the National Cancer Institute, vol. 102, pp. 161-169, Feb 32010.

[21] J. E. Joy, E. E. Penhoet, D. B. Petitti. Committee on new approaches to early detection and diagnosis of breast cancer, policy and global affairs, national research council (U.S.), Board on science technology and economic policy., Saving women's lives : strategies for improving breast cancer detection and diagnosis. Washington, D.C.: National Academies Press, 2005.

[22] R. J. Bleicher, R. M. Ciocca, B. L. Egleston, L. Sesa, K. Evers, E. R. Sigurdson, and M. Morrow, "Association of routine pretreatment magnetic resonance imaging with time to surgery, mastectomy rate, and margin status," Journal of the American College of Surgeons, vol. 209, pp. 180-187, Aug 2009. 
[23] "American cancer society. Breast cancer facts \& figures 2011-2012," Atlanta: American cancer society 2011.

[24] M. Pilewskie, C. Kennedy, C. McGathey, I. B. Helenowski, K. Bethke, N. Hansen, J. Jeruss, and S. A. Khan, "Effect of mri on the management of ductal carcinoma in situ (dcis) of the breast," Annals of Surgical Oncology, vol. 19, pp. S11-S11, Feb 2012.

[25] K. K. Lindfors, J. M. Boone, T. R. Nelson, K. Yang, A. L. C. Kwan, and D. F. Miller, "Dedicated breast CT: Initial clinical experience," Radiology, vol. 246, pp. 725-733, Mar 2008.

[26] N. D. Prionas, S. Y. Huang, and J. M. Boone, "Experimentally determined spectral optimization for dedicated breast computed tomography," Medical Physics, vol. 38, pp. 646655, Feb 2011.

[27] J. M. Boone, T. R. Nelson, K. K. Lindfors, and J. A. Seibert, "Dedicated breast CT: Radiation dose and image quality evaluation," Radiology, vol. 221, pp. 657-667, Dec 2001.

[28] R. Lavayssiere, A. E. Cabee, and J. E. Filmont, "Positron Emission Tomography (PET) and breast cancer in clinical practice," European Journal of Radiology, vol. 69, pp. 50-58, Jan 2009.

[29] K. Schilling, D. Narayanan, J. E. Kalinyak, J. The, M. V. Velasquez, S. Kahn, M. Saady, R. Mahal, and L. Chrystal, "Positron emission mammography in breast cancer presurgical planning: comparisons with magnetic resonance imaging," European Journal of Nuclear Medicine and Molecular Imaging, vol. 38, pp. 23-36, Jan 2011.

[30] C. Passmann and H. Ermert, "In vivo imaging of the skin in the $100 \mathrm{MHz}$ region using the synthetic aperture concept," 1995 IEEE Ultrasonics Symposium Proceedings, Vols 1 and 2, pp. 1287-1290, 1995.

[31] D. A. Christensen, Ultrasonic bioinstrumentation. New York: Wiley, 1988.

[32] B. S. Garra, E. I. Cespedes, J. Ophir, S. R. Spratt, R. A. Zuurbier, C. M. Magnant, and M. F. Pennanen, "Elastography of breast lesions: Initial clinical results," Radiology, vol. 202, pp. 79-86, Jan 1997.

[33] R. L. Ehman and J. F. Greenleaf, "Magnetic-resonance elastography by direct visualization of propagating acoustic strain waves (Vol 269, Pg 1854, 1995)," Science, vol. 270, pp. 565565, Oct 271995. 
[34] J. Ophir, I. Cespedes, H. Ponnekanti, Y. Yazdi, and X. Li, "Elastography - a quantitative method for imaging the elasticity of biological tissues," Ultrasonic Imaging, vol. 13, pp. 111-134, Apr 1991.

[35] I. Cespedes and J. Ophir, "Reduction of image noise in elastography," Ultrasonic Imaging, vol. 15, pp. 89-102, Apr 1993.

[36] M. M. Doyley, "Model-based elastography: a survey of approaches to the inverse elasticity problem," Physics in Medicine and Biology, vol. 57, pp. R35-73, Feb 72012.

[37] R. G. Barr, "Sonographic breast elastography," Journal of Ultrasound in Medicine, vol. 31, pp. 773-783, May 2012.

[38] "World cancer report," International agency for research on cancer (IARC)2008.

[39] "American cancer society. Breast cancer facts \& figures 2011-2012," Atlanta: American Cancer Society, Inc. 2011.

[40] R. Siegel, D. Naishadham, and A. Jemal, "Cancer statistics, 2013," Ca-a Cancer Journal for Clinicians, vol. 63, pp. 11-30, Jan-Feb 2013.

[41] N. Houssami, S. J. Lord, and S. Ciatto, "Breast cancer screening: emerging role of new imaging techniques as adjuncts to mammography," Medical Journal of Australia, vol. 190, pp. 493-498, May 42009.

[42] P. A. Carney, D. L. Miglioretti, B. C. Yankaskas, K. Kerlikowske, R. Rosenberg, C. M. Rutter, B. M. Geller, L. A. Abraham, S. H. Taplin, M. Dignan, G. Cutter, and R. BallardBarbash, "Individual and combined effects of age, breast density, and hormone replacement therapy use on the accuracy of screening mammography," Annals of Internal Medicine, vol. 138, pp. 168-175, Feb 42003.

[43] S. P. Poplack, P. A. Carney, J. E. Weiss, L. Titus-Ernstoff, M. E. Goodrich, and A. N. A. Tosteson, "Screening mammography: Costs and use of screening-related services," Radiology, vol. 234, pp. 79-85, Jan 2005.

[44] S. Goenezen, J. F. Dord, Z. Sink, P. E. Barbone, J. F. Jiang, T. J. Hall, and A. A. Oberai, "Linear and nonlinear elastic modulus imaging: An application to breast cancer diagnosis," IEEE Transactions on Medical Imaging, vol. 31, pp. 1628-1637, Aug 2012.

[45] M. M. Doyley, J. C. Bamber, F. Fuechsel, and N. L. Bush, "A freehand elastographic imaging approach for clinical breast imaging: System development and performance evaluation," Ultrasound in Medicine and Biology, vol. 27, pp. 1347-1357, Oct 2001. 
[46] R. J. Housden, A. H. Gee, G. M. Treece, and R. W. Prager, "3-D Ultrasonic strain imaging using freehand scanning and a mechanically-swept probe," IEEE Transactions on Ultrasonics Ferroelectrics and Frequency Control, vol. 57, pp. 501-506, Feb 2010.

[47] M. A. Lubinski, S. Y. Emelianov, and M. O'Donnell, "Adaptive strain estimation using retrospective processing," IEEE Transactions on Ultrasonics Ferroelectrics and Frequency Control, vol. 46, pp. 97-107, Jan 1999.

[48] J. Ophir, S. Srinivasan, R. Righetti, and A. Thittai, "Elastography: A decade of progress (2000-2010)," Current Medical Imaging Reviews, vol. 7, pp. 292-312, Nov 2011.

[49] H. Y. Xu, M. Rao, T. Varghese, A. Sommer, S. Baker, T. J. Hall, G. A. Sisney, and E. S. Burnside, "Axial-shear strain imaging for differentiating benign and malignant breast masses," Ultrasound in Medicine and Biology, vol. 36, pp. 1813-1824, Nov 2010.

[50] E. E. Konofagou, T. Harrigan, and J. Ophir, "Shear strain estimation and lesion mobility assessment in elastography," Ultrasonics, vol. 38, pp. 400-404, Mar 2000.

[51] A. Thitaikumar, R. Righetti, T. A. Krouskop, and J. Ophir, "Resolution of axial shear strain elastography," Physics in Medicine and Biology, vol. 51, pp. 5245-5257, Oct 212006.

[52] A. V. Patil, C. D. Garson, and J. A. Hossack, "3D prostate elastography: algorithm, simulations and experiments," Physics in Medicine and Biology, vol. 52, pp. 3643-3663, Jun 212007.

[53] R. Zahiri-Azar and S. E. Salcudean, "Motion estimation in ultrasound images using time domain cross correlation with prior estimates," IEEE Transactions on Biomedical Engineering, vol. 53, pp. 1990-2000, Oct 2006.

[54] R. Zahiri-Azar and S. E. Salcudean, "Time-delay estimation in ultrasound echo signals using individual sample tracking," IEEE Transactions on Ultrasonics Ferroelectrics and Frequency Control, vol. 55, pp. 2640-2650, Dec 2008.

[55] H. Chen and T. Varghese, "Multilevel hybrid 2D strain imaging algorithm for ultrasound sector/phased arrays," Medical Physics, vol. 36, pp. 2098-2106, Jun 2009.

[56] K. Liu, P. F. Zhang, J. H. Shao, X. J. Zhu, Y. Zhang, and J. Bai, "A 2D strain estimator with numerical optimization method for soft-tissue elastography," Ultrasonics, vol. 49, pp. 723732, Dec 2009. 
[57] S. K. Alam, J. Ophir, and E. E. Konofagou, "An adaptive strain estimator for elastography," IEEE Transactions on Ultrasonics Ferroelectrics and Frequency Control, vol. 45, pp. 461472, Mar 1998.

[58] S. Srinivasan and J. Ophir, "A zero-crossing strain estimator for elastography," Ultrasound in Medicine and Biology, vol. 29, pp. 227-38, Feb 2003.

[59] P. Chaturvedi, M. F. Insana, and T. J. Hall, "2-D companding for noise reduction in strain imaging," IEEE Trans Ultrason Ferroelectr Freq Control, vol. 45, pp. 179-91, 1998.

[60] T. Varghese, M. Bilgen, and J. Ophir, "Multiresolution imaging in elastography," IEEE Trans Ultrason Ferroelectr Freq Control, vol. 45, pp. 65-75, 1998.

[61] E. Konofagou and J. Ophir, "A new elastographic method for estimation and imaging of lateral displacements, lateral strains, corrected axial strains and Poisson's ratios in tissues," Ultrasound in Medicine and Biology, vol. 24, pp. 1183-1199, Oct 1998.

[62] S. Srinivasan, J. Ophir, and S. K. Alam, "Elastographic imaging using staggered strain estimates," Ultrasonic Imaging, vol. 24, pp. 229-245, Oct 2002.

[63] F. Kallel and J. Ophir, "A least-squares strain estimator for elastography," Ultrasonic Imaging, vol. 19, pp. 195-208, Jul 1997.

[64] S. Srinivasan, J. Ophir, and S. K. Alam, "Theoretical derivation of SNR, CNR and spatial resolution for a local adaptive strain estimator for elastography," Ultrasound in Medicine and Biology, vol. 30, pp. 1185-1197, Sep 2004.

[65] M. Bilgen, S. Srinivasan, L. B. Lachman, and J. Ophir, "Elastography imaging of small animal oncology models: A feasibility study," Ultrasound in Medicine and Biology, vol. 29, pp. 1291-1296, Sep 2003.

[66] S. Bharat, T. G. Fisher, T. Varghese, T. J. Hall, J. Jiang, E. L. Madsen, J. A. Zagzebski, and F. T. Lee, "Three-dimensional electrode displacement elastography using the siemens C7F2 foursight four-dimensional ultrasound transducer," Ultrasound in Medicine and Biology, vol. 34, pp. 1307-1316, Aug 2008.

[67] J. F. Deprez, E. Brusseau, C. Schmitt, G. Cloutier, and O. Basset, "3D estimation of soft biological tissue deformation from radio-frequency ultrasound volume acquisitions," Medical Image Analysis, vol. 13, pp. 116-127, Feb 2009. 
[68] G. M. Treece, J. E. Lindop, A. H. Gee, and R. W. Prager, "Freehand ultrasound elastography with a 3-D probe," Ultrasound in Medicine and Biology, vol. 34, pp. 463-474, Mar 2008.

[69] M. S. Richards, P. E. Barbone, and A. A. Oberai, "Quantitative three-dimensional elasticity imaging from quasi-static deformation: a phantom study," Physics in Medicine and Biology, vol. 54, pp. 757-779, Feb 72009.

[70] C. Xu and J. L. Prince, "Snakes, shapes, and gradient vector flow," IEEE Trans Image Process, vol. 7, pp. 359-69, 1998.

[71] S. Osher and J. A. Sethian, "Fronts propagating with curvature-dependent speed - algorithms based on hamilton-jacobi formulations," Journal of Computational Physics, vol. 79, pp. 1249, Nov 1988.

[72] M. Kass, A. Witkin, and D. Terzopoulos, "Snakes - active contour models," International Journal of Computer Vision, vol. 1, pp. 321-331, 1987.

[73] E. Street, L. Hadjiiski, B. Sahiner, S. Gujar, M. Ibrahim, S. K. Mukherji, and H. P. Chan, "Automated volume analysis of head and neck lesions on CT scans using 3D level set segmentation," Medical Physics, vol. 34, pp. 4399-4408, Nov 2007.

[74] E. D. Angelini, S. Homma, G. Pearson, J. W. Holmes, and A. F. Laine, "Segmentation of real-time three-dimensional ultrasound for quantification of ventricular function: A clinical study on right and left ventricles," Ultrasound in Medicine and Biology, vol. 31, pp. 11431158, Sep 2005.

[75] W. K. Moon, R. F. Chang, C. J. Chen, D. R. Chen, and W. L. Chen, "Solid breast masses: Classification with computer-aided analysis of continuous US images obtained with probe compression," Radiology, vol. 236, pp. 458-464, Aug 2005.

[76] C. M. Li, C. Y. Xu, C. F. Gui, and M. D. Fox, "Distance regularized level set evolution and its application to image segmentation," IEEE Transactions on Image Processing, vol. 19, pp. 3243-3254, Dec 2010.

[77] W. Liu, J. A. Zagzebski, T. Varghese, C. R. Dyer, U. Techavipoo, and T. J. Hall, "Segmentation of elastographic images using a coarse-to-fine active contour model," Ultrasound in Medicine and Biology, vol. 32, pp. 397-408, Mar 2006.

[78] Z. Yang, S. P. Sinha, R. C. Booi, M. A. Roubidoux, B. Ma, J. B. Fowlkes, G. L. LeCarpentier, and P. L. Carson, "Breast ultrasound image improvement by pixel 
compounding of compression sequence," IEEE Transactions on Ultrasonics Ferroelectrics and Frequency Control, vol. 56, pp. 465-473, Mar 2009.

[79] S. Selvan, M. Kavitha, S. S. Devi, and S. Suresh, "Fuzzy-based classification of breast lesions using ultrasound echography and elastography," Ultrasound Quarterly, vol. 28, pp. 159-167, Sep 2012.

[80] J. Ophir, S. K. Alam, B. Garra, F. Kallel, E. Konofagou, T. Krouskop, and T. Varghese, "Elastography: ultrasonic estimation and imaging of the elastic properties of tissues," Proc Inst Mech Eng H, vol. 213, pp. 203-33, 1999.

[81] H. Zhi, X. Y. Xiao, H. Y. Yang, B. Ou, Y. L. Wen, and B. M. Luo, "Ultrasonic elastography in breast cancer diagnosis: Strain ratio vs 5-point scale," Academic Radiology, vol. 17, pp. 1227-1233, Oct 2010.

[82] F. K. W. Schaefer, I. Heer, P. J. Schaefer, C. Mundhenke, S. Osterholz, B. M. Order, N. Hofheinz, J. Hedderich, M. Heller, W. Jonat, and I. Schreer, "Breast ultrasound elastography-Results of 193 breast lesions in a prospective study with histopathologic correlation," European Journal of Radiology, vol. 77, pp. 450-456, Mar 2011.

[83] D. Kotsianos-Hermle, K. M. Hiltawsky, S. Wirth, T. Fischer, K. Friese, and M. Reiser, "Analysis of 107 breast lesions with automated 3D ultrasound and comparison with mammography and manual ultrasound," European Journal of Radiology, vol. 71, pp. 109115, Jul 2009.

[84] T. J. Hall, Y. N. Zhu, and C. S. Spalding, "In vivo real-time freehand palpation imaging," Ultrasound in Medicine and Biology, vol. 29, pp. 427-435, 2003.

[85] R. G. Barr, S. Destounis, L. B. Lackey, 2nd, W. E. Svensson, C. Balleyguier, and C. Smith, "Evaluation of breast lesions using sonographic elasticity imaging: a multicenter trial," $J$ Ultrasound Med, vol. 31, pp. 281-7, Feb 2012.

[86] S. Y. Emelianov, R. Q. Erkamp, M. A. Lubinski, A. R. Skovoroda, and M. O'Donnell, "Non-linear tissue elasticity: Adaptive elasticity imaging for large deformations," 1998 IEEE Ultrasonics Symposium - Proceedings, Vols 1 and 2, pp. 1753-1756, 1998.

[87] J. P. Xu, S. Tripathy, J. M. Rubin, R. W. Stidham, L. A. Johnson, P. D. R. Higgins, and K. Kim, "A new nonlinear parameter in the developed strain-to-applied strain of the soft tissues and Its application in ultrasound elasticity imaging," Ultrasound in Medicine and Biology, vol. 38, pp. 511-523, Mar 2012. 
[88] T. Varghese, J. Ophir, and T. A. Krouskop, "Nonlinear stress-strain relationships in tissue and their effect on the contrast-to-noise ratio in elastograms," Ultrasound in Medicine and Biology, vol. 26, pp. 839-851, Jun 2000.

[89] A. A. Oberai, N. H. Gokhale, S. Goenezen, P. E. Barbone, T. J. Hall, A. M. Sommer, and J. F. Jiang, "Linear and nonlinear elasticity imaging of soft tissue in vivo: demonstration of feasibility," Physics in Medicine and Biology, vol. 54, pp. 1191-1207, Mar 72009.

[90] S. Goenezen, J. F. Dord, Z. Sink, P. E. Barbone, J. Jiang, T. J. Hall, and A. A. Oberai, "Linear and nonlinear elastic modulus imaging: an application to breast cancer diagnosis," IEEE Trans Med Imaging, vol. 31, pp. 1628-37, Aug 2012.

[91] Y. P. Qiu, M. Sridhar, J. K. Tsou, K. K. Lindfors, and M. F. Insana, "Ultrasonic viscoelasticity imaging of nonpalpable breast tumors: preliminary results," Academic Radiology, vol. 15, pp. 1526-1533, Dec 2008.

[92] M. Sridhar and M. F. Insana, "Ultrasonic measurements of breast viscoelasticity," Medical Physics, vol. 34, pp. 4757-4767, Dec 2007.

[93] A. Thitaikumar, T. A. Krouskop, B. S. Garra, and J. Ophir, "Visualization of bonding at an inclusion boundary using axial-shear strain elastography: a feasibility study," Physics in Medicine and Biology, vol. 52, pp. 2615-2633, May 72007.

[94] L. J. Chen, R. J. Housden, G. M. Treece, A. H. Gee, and R. W. Prager, "A normalization method for axial-shear strain elastography," IEEE Transactions on Ultrasonics Ferroelectrics and Frequency Control, vol. 57, pp. 2833-2838, Dec 2010.

[95] A. K. Thittai, J. M. Yamal, L. M. Mobbs, C. M. Kraemer-Chant, S. Chekuri, B. S. Garra, and J. Ophir, "Axial-shear strain elastography for breast lesion classification: Further results from in vivo data," Ultrasound in Medicine and Biology, vol. 37, pp. 189-197, Feb 2011.

[96] A. Thitaikumar, L. M. Mobbs, C. M. Kraemer-Chant, B. S. Garra, and J. Ophir, "Breast tumor classification using axial shear strain elastography: a feasibility study," Physics in Medicine and Biology, vol. 53, pp. 4809-4823, Sep 72008.

[97] U. Techavipoo, Q. Chen, T. Varghese, and J. A. Zagzebski, "Estimation of displacement vectors and strain tensors in elastography using angular insonifications," IEEE Transactions on Medical Imaging, vol. 23, pp. 1479-1489, Dec 2004.

[98] S. J. Orfanidis, Optimum signal processing an Introduction, Second Edition ed.: Sophocles J. Orfanidis, 2007. 
[99] R. Righetti, J. Ophir, and P. Ktonas, "Axial resolution in elastography," Ultrasound in Medicine and Biology, vol. 28, pp. 101-113, Jan 2002.

[100] R. G. P. Lopata, M. M. Nillesen, H. H. G. Hansen, I. H. Gerrits, J. M. Thijssen, and C. L. de Korte, "Performance evaluation of methods for two-dimensional displacement and strain estimation using ultrasound radio frequency data," Ultrasound in Medicine and Biology, vol. 35, pp. 796-812, May 2009.

[101] A. Thitaikumar, T. A. Krouskop, and J. Ophir, "Signal-to-noise ratio, contrast-to-noise ratio and their trade-offs with resolution in axial-shear strain elastography," Physics in Medicine and Biology, vol. 52, pp. 13-28, Jan 72007.

[102] R. C. Hibbeler, Mechanics of materials, 8th ed. Upper Saddle River, N.J.: Pearson Prentice Hall, 2011.

[103] C. O. Horgan, G. Saccomandi, and I. Sgura, "A two-point boundary-value problem for the axial shear of hardening isotropic incompressible nonlinearly elastic materials," Siam Journal on Applied Mathematics, vol. 62, pp. 1712-1727, Jul 32002.

[104] Serope Kalpakjian and S. Schmid, Manufacturing processes for engineering materials, 5 ed.: Prentice Hall, 2007

[105] S. C. Zhu and A. Yuille, "Region competition: Unifying snakes, region growing, and Bayes/MDL for multiband image segmentation," IEEE Transactions on Pattern Analysis and Machine Intelligence, vol. 18, pp. 884-900, Sep 1996.

[106] Breast elastography phantom model 059 data sheet. CIRS, Norfolk, VA, 2011.

[107] H. Ponnekanti, J. Ophir, Y. Huang, and I. Cespedes, "Fundamental mechanical limitations on the visualization of elasticity contrast in elastography," Ultrasound in Medicine and Biology, vol. 21, pp. 533-543, 1995.

[108] F. Kallel, M. Bertrand, and J. Ophir, "Fundamental limitations on the contrast-transfer efficiency in elastography: an analytic study," Ultrasound in Medicine and Biology, vol. 22, pp. 463-470, 1996.

[109] F. Kallel, C. D. Prihoda, and J. Ophir, "Contrast-transfer efficiency for continuously varying tissue moduli: Simulation and phantom validation," Ultrasound in Medicine and Biology, vol. 27, pp. 1115-1125, Aug 2001. 
[110] S. Srinivasan, F. Kallel, and J. Ophir, "Estimating the elastographic signal-to-noise ratio using correlation coefficients," Ultrasound in Medicine and Biology, vol. 28, pp. 359-368, Mar 2002.

[111] U. Techavipoo and T. Varghese, "Wavelet denoising of displacement estimates in elastography," Ultrasound in Medicine and Biology, vol. 30, pp. 477-491, Apr 2004.

[112] R. Chandrasekhar, J. Ophir, T. Krouskop, and K. Ophir, "Elastographic image quality vs. tissue motion in vivo," Ultrasound in Medicine and Biology, vol. 32, pp. 847-855, Jun 2006.

[113] A. Sayed, G. Layne, J. Abraham, and O. M. Mukdadi, "Nonlinear characterization of breast cancer using multi-compression 3d ultrasound elastography in vivo," Ultrasonics vol. 53, pp. 979-991, July 2013.

[114] T. J. Hall, Y. N. Zhu, C. S. Spalding, and L. T. Cook, "In vivo real-time freehand elasticity imaging," 2002 IEEE International Symposium on Biomedical Imaging, Proceedings, pp. 805-808, 2002.

[115] R. Mcgill, J. W. Tukey, and W. A. Larsen, "Variations of box plots," American Statistician, vol. 32, pp. 12-16, 1978.

[116] D. T. Ginat, S. V. Destounis, R. G. Barr, B. Castaneda, J. G. Strang, and D. J. Rubens, "Us elastography of breast and prostate lesions," Radiographics, vol. 29, pp. 2007-U195, Nov 2009.

[117] D. M. Regner, G. K. Hesley, N. J. Hangiandreou, M. J. Morton, M. R. Nordland, D. D. Meixner, T. J. Hall, M. A. Farrell, J. N. Mandrekar, W. S. Harmsen, and J. W. Charboneau, "Breast lesions: evaluation with us strain imaging - clinical experience of multiple observers," Radiology, vol. 238, pp. 425-437, Feb 2006.

[118] W. K. Moon, S. C. Chang, C. S. Huang, and R. F. Chang, "Breast tumor classification using fuzzy clustering for breast elastography," Ultrasound in Medicine and Biology, vol. 37, pp. 700-708, May 2011.

[119] S. B. Edge, D. R. Byrd, C. C. Compton, A. G. Fritz, F. L. Greene, and A. Trotti, American Joint Committee on Cancer (AJCC) cancer staging manual, 7th ed. New York ; London: Springer, 2010.

[120] S. L. Chappy, "Women's experience with breast biopsy," AORN journal, vol. 80, pp. 885901, 2004. 
[121] D. L. Weaver, P. M. Vacek, J. M. Skelly, and B. M. Geller, "Predicting biopsy outcome after mammography: what is the likelihood the patient has invasive or in situ breast cancer?," Annals of Surgical Oncology, vol. 12, pp. 660-73, 2005. 